\title{
Heart-rate Variability Analysis for Stress Assessment in a Video-Game Setup
}

\author{
By: \\ Syem Ishaque \\ A thesis \\ presented to Ryerson University \\ in partial fulfillment of the \\ requirements for the degree \\ of Master of Applied Science \\ in the program of \\ Biomedical Engineering
}

Bachelor of Engineering, Ryerson University, Toronto, 2017

Toronto, Ontario, Canada, 2020

(C)Syem Ishaque, 2020 


\section{AUTHOR'S DECLARATION FOR ELECTRONIC SUBMISSION OF A THESIS}

I hereby declare that I am the sole author of this thesis.

This is a true copy of the thesis, including any required final revisions, as accepted by my examiners.

I authorize Ryerson University to lend this thesis to other institutions or individuals for the purpose of scholarly research.

I further authorize Ryerson University to reproduce this thesis by photocopying or by other means, in total or in part, at the request of other institutions or individuals for the purpose of scholarly research.

I understand that my thesis may be made electronically available to the public. 
Heart Rate Variability Analysis for Stress Assessment in a Video-Game Setup, Syem Ishaque, Master of Science, Biomedical Engineering, Ryerson University, 2020

\begin{abstract}
Chronic stress makes a person vulnerable to diseases leading to the evolution of various physical and mental health conditions including chronic fatigue, diabetes, obesity, depression and other symptoms associated with immune disorders. This study investigates the impact of virtual reality video games to reduce stress and increase resistance to stress. The study consisted of 4 phases: (1) a baseline phase which was used to assess the subjects normal physiological function, (2) a virtual reality roller coaster phase which was meant to induce stress through the elicitation of affective emotions (3) a cognitive color stroop test was also used to induce stress and increase anxiety, and (4) a VR fish game was required to understand the impact of playing video games to reduce stress and anxiety. The physiological variation associated with stress was analyzed through physiological signals such as Electrocardiogram (ECG), respiratory signal (RESP), and Galvanic Skin Response (GSR). Specific features such as $p N N 50, R M S S D, A p E n, L F, H F$, $L F / H F$ ratio and respiration rate were extracted from the corresponding signals. ECG derived features were used as input features for various machine learning models. Poincare plots were used to indicate abnormal HRV and stress through a visual representation. SD1 and SD2 share a high correlation with SNS and PNS activity, $A p E n$ shares a 0.811 correlation with $L F / H F$ ratio, proving that it is an effective method to assess stress. Average results from the users indicate that $L F / H F$ reduced from 1.2 to $0.93, A p E n$ reduced from 0.0 .695 to 0.562 after playing the VR fish game, demonstrating the positive influence of the game towards stress reduction. Statistical analysis using the t-test verified that the data were statistically significant, p-value $<0.05$ rejected that the null hypothesis that the data was statistically significant. The Ensemble Gradient Boosting model was able to classify binary classes associated with stress/relaxation with 100\% accuracy, various other models were able to classify with $90 \%$ accuracy. RUC curve, precision/recall curve and $T P, F P, T N, F N$ revealed that few models such as Naive Bayes were inadequate for stress classification. The research study effectively demonstrated the impact of VR fish game for stress reduction.
\end{abstract}




\section{Acknowledgements}

First and foremost, I would like to thank my supervisor Dr. Sri Krishnan for his guidance on this great journey which began two years earlier. He really set the tone, provided valuable feedback, and directed me to the finish line. I am grateful for his leadership and guidance.

I would also like to thank my co-supervisor Dr. Naimul Khan, who was responsible for directing the Shaftesbury project which provided all the data for this thesis. He also provided valuable feedback which helped complete my thesis and produce desired results. This wouldn't have been possible without his leadership and guidance

I would also like to thank Shaftsbury who provided us with bubble bloom VR video game.

I would like to thank my fellow partners from the Shaftesbury project as well for their feedback and guidance.

First of all, I would like to thank Suhana Rabbani who collected the data for the experiment, provided valuable feedback and also assisted for other tasks required for the completion of the thesis. I wouldn't have been to complete the thesis without her effort.

I also would like to thank other members of the group from RML lab, Nabila Abraham, Rehman Zafar, Zeeshan Ahmad for their feedback and effort for the project.

I would also like to thank all the members of the SAR Lab for their warm support.

I would like to thank Sourav Kumar for his feedback and motivation. I would also like to thank Dharmendra Guru, Alice Rueda and Garima Sharma for their support and feedback.

I would like to thank my parents and brother for their support and love. They really gave me the boost to work diligently and focus, as well as providing empowering motivation. 


\section{Table of Contents}

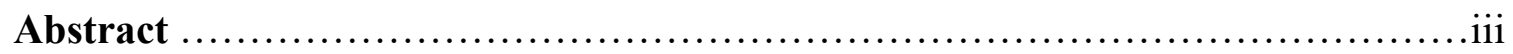

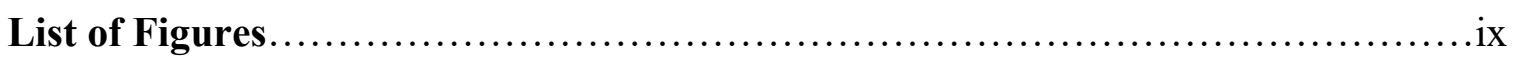

List of Tables.....................................................................

\section{Introduction}

1.1. Introduction............................................................

1.2. Method ...............................................................

1.3. Organization of the Paper............................................

1. Introduction ................................................................ 8

2. Literature Review ........................................................... 8

3. Physiological Signal Analysis ......................................... 8

4. Machine Learning ..................................................... 8

5. Conclusion .............................................................. 9

2. Literature Review..................................................... 10

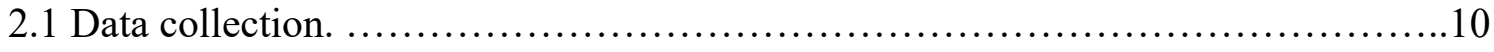

2.2. Feature Extraction....................................................... 11

2.3. Wearable Devices..................................................11

2.4 Machine Learning................................................... 13

2.4a Convolutional Neural Network (CNN) used to detect stress through HRV ..........13

$2.4 \mathrm{~b}$ Ultra Short periods to assess HRV/stress ...................................... 16

2.4c. Classifying HRV from ECG and EDA features through ML classification

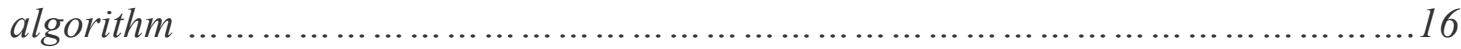

2.4d. Abnormal HRV classified remotely ........................................... 17

2.4e. HRV associated with affective computing, classified through NN and SVM..... 19

2.4f. Stress Classification through HRV analysis ....................................20

2.4g. Contradictions associated with stress classification and analysis using features

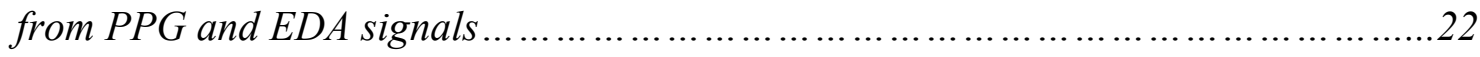

2.4h. Stress induced through VR environment and classified using Extreme learning machine (ELM) 


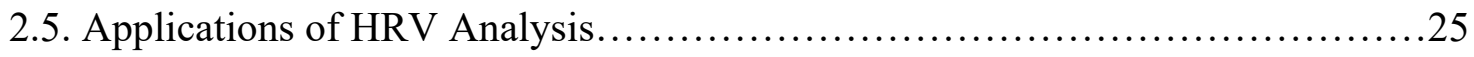

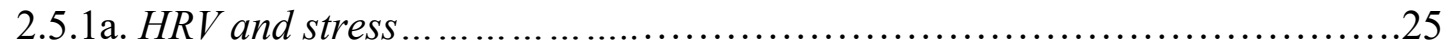

2.5.1b. Low/Reduced HRV ................................................27

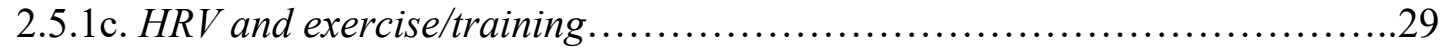

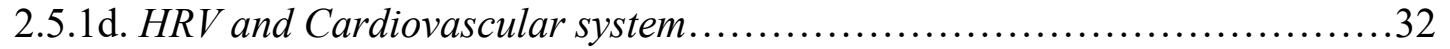

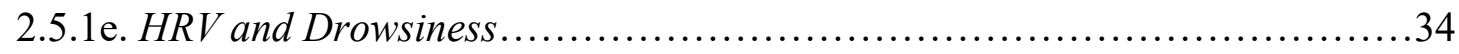

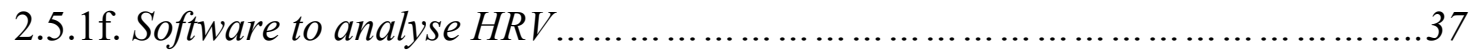

2.5.1g. Bridging the gap between Human physiology and video game......................38

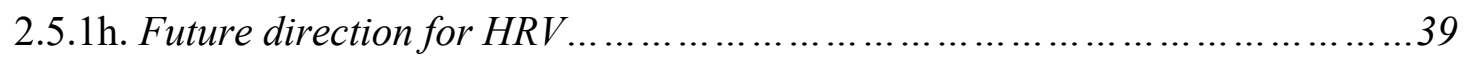

2.6. Stress and Video games................................................. 40

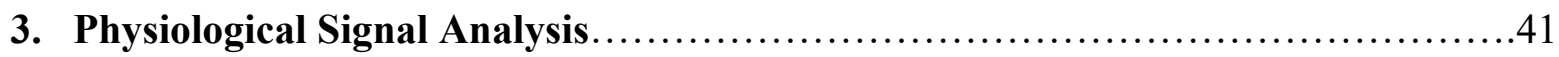

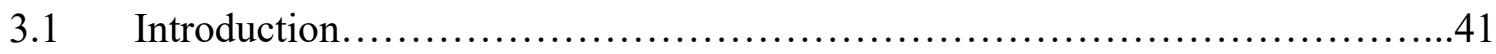

3.1.1 Physiological Signal Analysis........................................... 42

3.1.2 Non-Linear Analysis, GSR, Respiration..................................45

3.1.3 Power Spectral Density..................................................... 46

3.1.4 Preprocessing/Filtering .................................................. 48

$3.2 \quad$ Method/Implementation................................................ 49

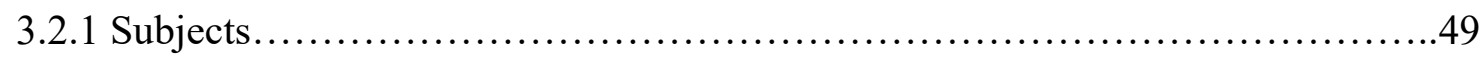

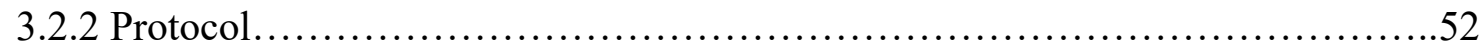

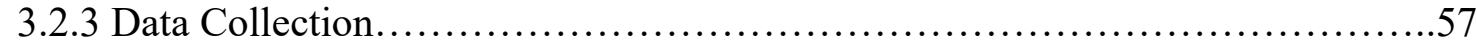

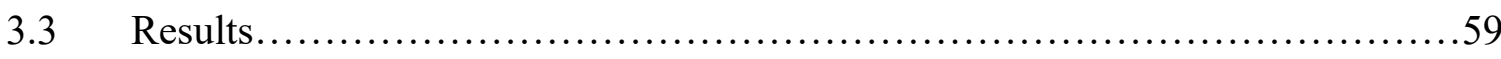

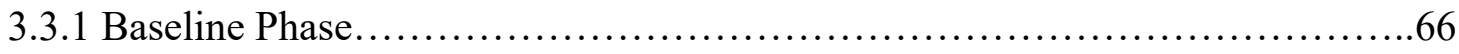

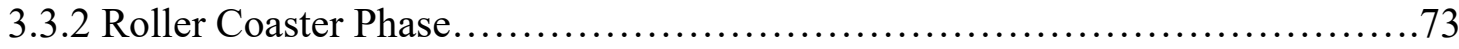

3.3.3 Cognitive Color Stroop Test................................................ 79

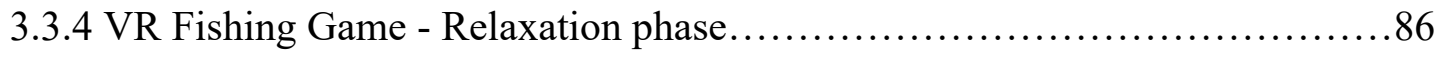

4. Classification of Stress through Machine Learning ............................99

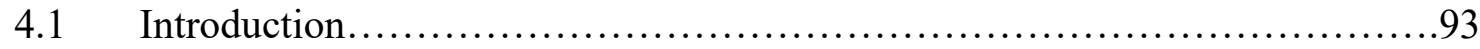

4.1.1 Supervised Learning/ Machine Learning Models: .............................94 


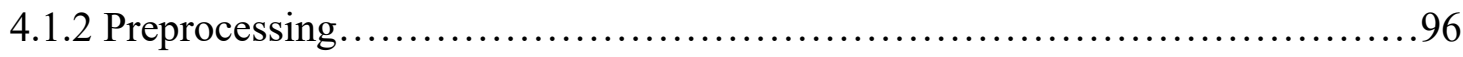

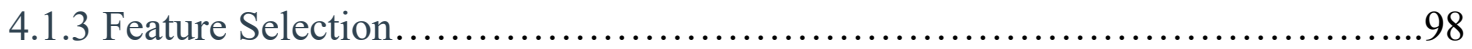

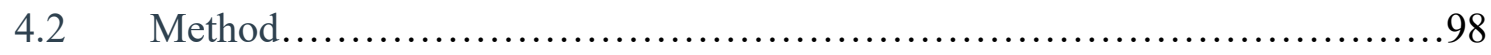

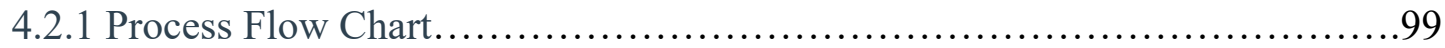

4.2.2 F1, Precision, Recall, Auc............................................... 100

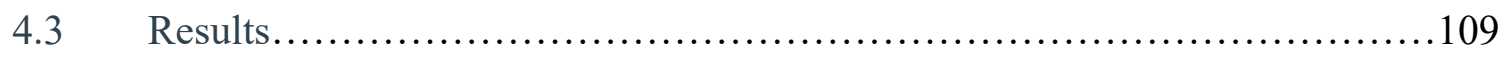

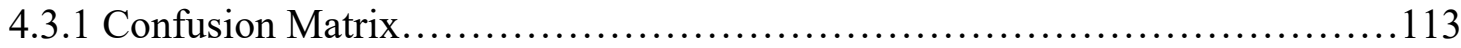

4.3.2 Precision/Recall curves.............................................. 118

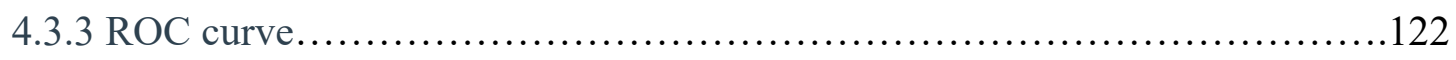

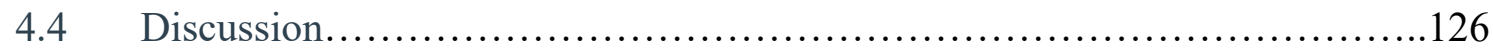

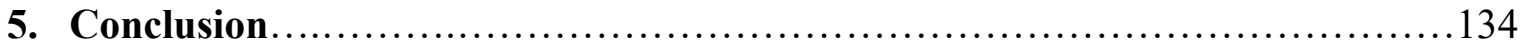

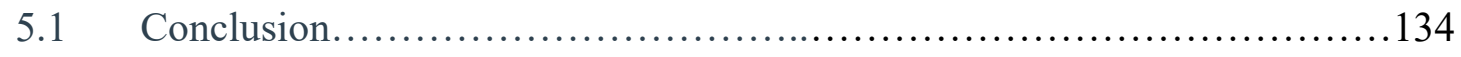

$5.2 \quad$ Limitations.............................................................. 136

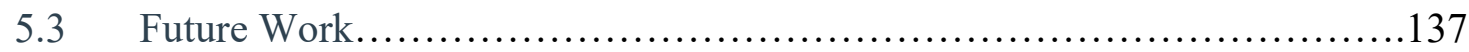

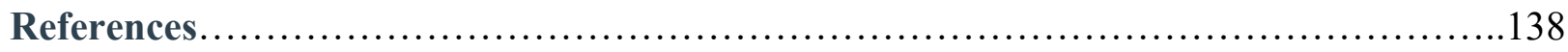




\section{List of Tables}

Table 1: Research papers associated with HRV detected using an ECG, type of study, results of HRV, concepts being analyzed. ..........................................................

Table 2: User 2 results obtained from baseline (T1) - VR fishing game (T4) phase. The p-value indicates if the corresponding results are significant (marked in red). .......................... 85 


\section{List of Figures}

Figure 1: Variations in $R-R$ intervals which represents HRV [73] . ...................

Figure 2: Block diagram which indicates the organization of the thesis................ 9

Figure 3: Wireless devices used to detect ECG, Respiration, GSR, skin temperature [72]..13

Figure 4: Activation difference for first five CNN layer between rest and stress state [70].

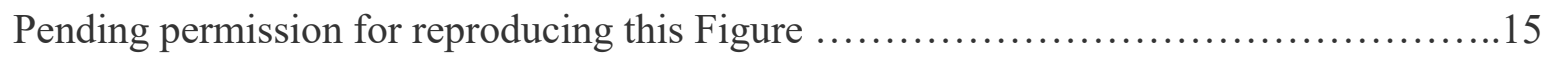

Figure 5: Telemonitoring of HRV through microwave doppler wireless radar [77], wireless

ECG/PPG device [78], wireless A/D converter [79], HRV analysis software and internet.19

Figure 6: Stress classification through machine learning flowchart.....................22

Figure 7: Describes $L F$ and $H F$ associated with stress, drowsiness, awake, fatigue [3]....26

Figure 8: Assessing training performance of athletes through HRV [75] ................ 30

Figure 9: PNS and SNS activity associated with HRV [71] ............................

Figure 10: Process behind drowsiness detection through HRV [3] ...................... 35

Figure 11: HRV analysis using Kubios Software................................ 37

Figure 12: A system to detect and analyze HRV [76] ............................. 38

Figure 13: Protocol for data collection from the four distinct phases: Baseline phase, VR Roller

Coaster Phase, Cognitive Stroop Task and VR Fish Game..........................50

Figure 14: Data acquisition through wireless ECG, GSR and RESP devices..............52

Figure 15: Notch Filter and 1D - Wavelet Decomposition Process Flow Chart for preprocessing

ECG signal (in preprocessing write a sentence to look here $\ldots \ldots \ldots \ldots \ldots \ldots \ldots \ldots \ldots \ldots . \ldots \ldots$

Figure 16: Process Flow Chart for plotting a poincare plot..........................53

Figure 17: Blackman Filter and 1D - Wavelet Decomposition Process Flow Chart for

preprocessing Respiration/EDR signal........................................54

Figure 18: Low Pass Butterworth filter and 8 - 30 seconds sliding windows Process Flow Chart

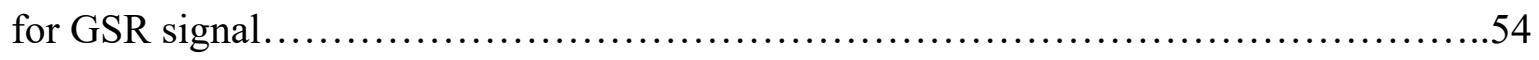

Figure 19: Design process used to analyze HRV in time and frequency domain..........55

Figure 20: Process behind analyzing HRV in Time Domain.........................55

Figure 21: Process utilized to analyze HRV in Frequency Domain through MATLAB...56

Figure 22: Visual Representation of Data Acquisition - Time/Frequency Domain Feature

Extraction and Analysis - Machine Learning Classification..........................57 
Figure 23: Sample raw ECG of a subject during baseline phase...........................59

Figure 24: Filtered ECG using a notch filter, during the baseline phase......................59

Figure 25: The signal is further smoothened to remove tapering noise, and make the peaks

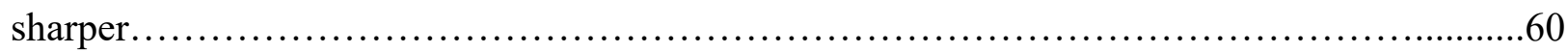

Figure 26: Detected R-peaks during the baseline phase................................61

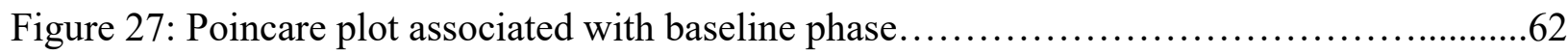

Figure 28: AR method used to delineate PSD during baseline phase. .......................63

Figure 29: PSD depiction using Lomb Scargle Periodogram during Baseline phase. ............64

Figure 30: Respiration signal associated with baseline phase ............................65

Figure 31: GSR obtained from baseline phase using 30 second window....................66

Figure 32: $R-R$ peaks detected during roller coaster phase (phase/T 2) .....................67

Figure 33: Poincare plot of user 12 during the roller coaster phase $\ldots \ldots \ldots \ldots \ldots \ldots \ldots \ldots \ldots \ldots . \ldots \ldots$

Figure 34: AR periodogram obtained from Roller coaster VR simulation. ....................69

Figure 35: Lomb Scargle periodogram obtained from VR roller coaster phase....................70

Figure 36: Respiration signal due to roller coaster phase. ............................. 71

Figure 37: GSR signals associated with Roller coaster phase. ..........................72

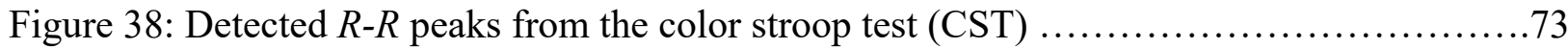

Figure 39: Visual representation of a stress and no stress phase from VR roller coaster

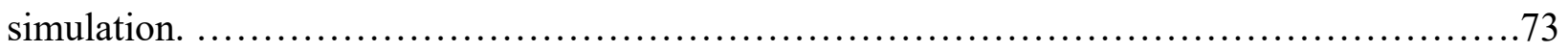

Figure 40: HRV signal which represents variation between Heart beats from CST. ............74

Figure 41: Poincare plot obtained from stroop task................................... 75

Figure 42: AR periodogram obtained from color stroop task............................76

Figure 43: Lomb Scargle Periodogram portraying ANS activity during stroop task............77

Figure 44: Respiration signal during color stroop task. BR- $15.7510 \ldots \ldots \ldots \ldots \ldots \ldots \ldots \ldots \ldots$

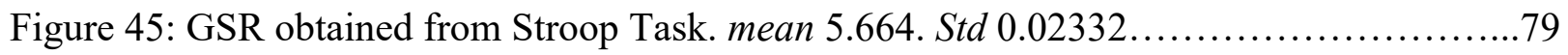

Figure 46: $R-R$ peaks obtained from VR fishing game................................ 80

Figure 47: Poincare plot which is indicative of normal physiological function.................80

Figure 48: AR periodogram obtained during VR fishing game. ......................... 81

Figure 49: Lomb Scargle periodogram due to VR fishing game. ........................ 82

Figure 50: Respiration signal during fishing game.................................... 83

Figure 51: GSR signals obtained from VR fishing game. ........................... 84 
Figure 52: Physical, physiological and behavioural symptoms associated with $L F, H F$ variations.

Figure 53: AR and Lomb Scargle periodogram PSD obtained during color troop task for User 10. .88

Figure 54: Visual Representation describing an abnormal and normal HRV on a poincare plot.89 Figure 55: Procedure used to classify stress/relax after data acquisition.

Figure 56: Process flow chart used to derive Ensemble Gradient Boosting classifier and evaluate the model. .98

Figure 57: Impact of Video Games on physiological function and stress levels.

Figure 58: Correlation map to understand which feature were the most significant for classifying stress. . . 101

Figure 59(a, b, c): Histogram of the Features used to classify stress through binary classification..

Figure 60: Box plot of features and stress labels used for binary classification. 105

Figure 61: Box plot of features and stress labels used for binary classification.................106

Figure 62: Box plot of features and stress label used for binary classification.................107

Figure 63: Box plot of features and stress label used for binary classification.................108

Figure 64: Confusion matrix for binary classification using DT ..........................109

Figure 65: Confusion matrix from binary classification of stress through LDA. .............110

Figure 66: Confusion matrix obtained from binary classification of stress through Gaussian

NB.

Figure 67: Confusion Matrix of binary classification obtained through KNN ...............112

Figure 68: Confusion matrix obtained through ensemble Gradient Boosting Classifier.........113

Figure 69: Precision and recall curve for binary classification of stress using decision tree....114

Figure 70: Precision and Recall curve obtained using ensemble Gradient Boosting classifier

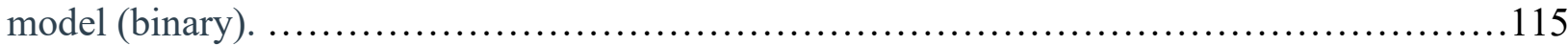

Figure 71: Precision and Recall curve obtained from KNN model (binary) ..................116

Figure 72: Precision and recall curve of Naive Bayes model (binary) .......................117

Figure 73: Precision and recall curve from LDA model (binary) ............................118

Figure 74: ROC curve obtained through ensemble Gradient Boosting classifier model.........119 
Figure 75: ROC curve obtained through LDA........................................... 120

Figure 76: ROC curve depicted from KNN model.........................................121

Figure 77: ROC curve produced from Naive Bayes model................................122

Figure 78: Classification accuracies obtained by each model following 5-Fold Cross

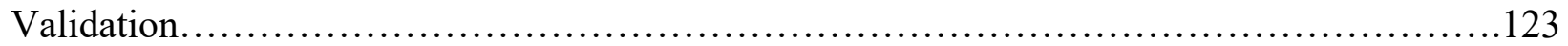

Figure 79: Mean Accuracies obtained by each model following 5-Fold Cross Validation........124

Figure 80: Standard Deviation obtained from each model following 5-Fold Cross

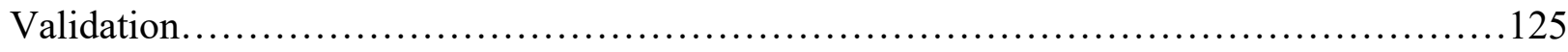

Figure 81: Evaluation of the performance of Naive Bayes model...........................127

Figure 82: Evaluation of the performance of ensemble Gradient Boosting Classifier model...128

Figure 83:Pie chart which displays the accuracy associated with various machine learning

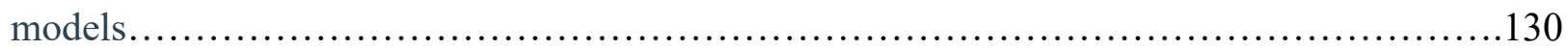

Figure 84: Mean accuracy and standard deviation obtained from 5-fold Cross Validation.......132 


\section{Chapter 1}

\section{Introduction}

\subsection{Introduction}

The human heart is an organ which is about the same size of a human fist, positioned right in the middle of the chest, faintly towards the left side [1]. It's functions include pumping blood to the tissues (located in different parts of the body such as the brain, lungs and other parts of the body) which supplies oxygen and nutrients to the tissues of the body and removes carbon dioxide and other waste products from the tissues by coordinating with the circulatory system (composed of heart, blood and blood vessels) and these activities are taking place with each heartbeat [1]. The heart is composed of three layers of tissue: the epicardium, myocardium and endocardium, the pericardium is a thin outer layer (outer layer of the heart) which protects the heart [1]. The heart is also made up of four chambers, two on each side of the heart. Left and right atria are the two small chambers positioned within the upper part of the heart, while the left and right ventricle are the two large chambers positioned within the lower part of the heart [1]. An ECG is often utilized to measure the activity of the heart, PPG (photoplethysmogram) which is used to detect blood volume changes can also be utilized but for most cardiac events ECG has proven to be an effective detection method. The activity of the heart is not constant, heart rate is around $65-70 \mathrm{bpm}$ (beats per minute) for a normal healthy adult and it varies due to the internal activity of the heart. HR is measured through the QRS complex obtained from an ECG wave, generally measured by detecting the $\mathrm{R}$ wave which is the most noticeable peak within the QRS complex. Each $R-R$ interval varies due to the variation associated with the activity of the heart due to normal/abnormal events, the variation in the time interval between each $R-R$ interval is known as heart rate variability (HRV) (as shown in Figure 1). 


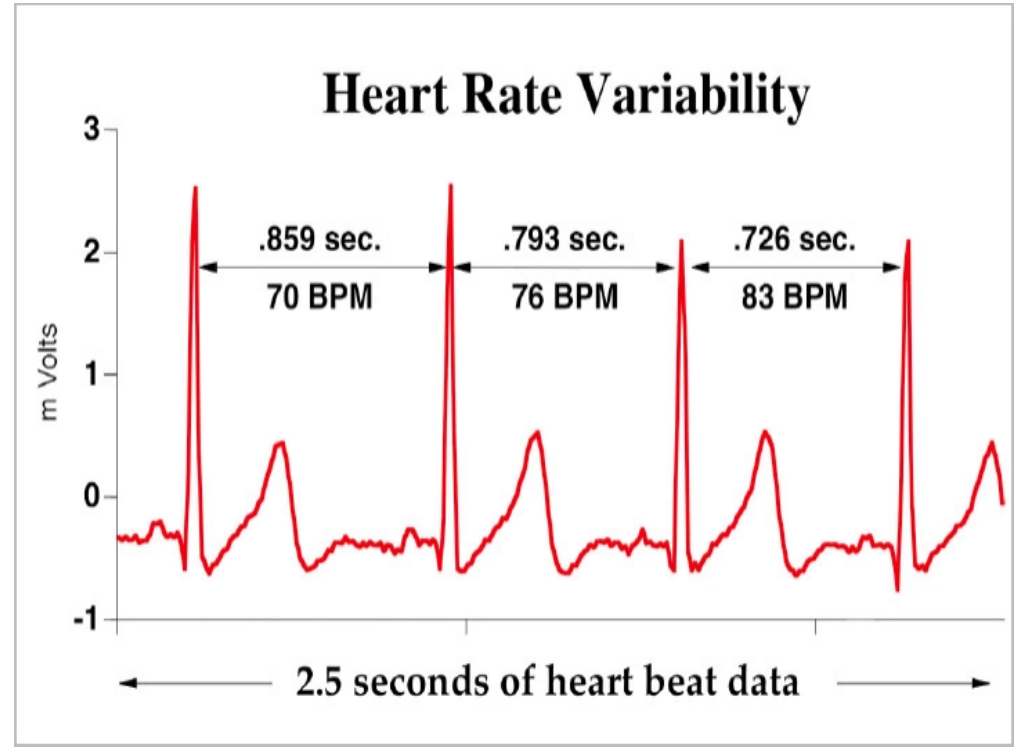

Figure 1: Variations in $R-R$ intervals which represents HRV [73].

HRV has been associated with many research studies involving morbidity and mortality, stress, fatigue, athletic performance. HRV primarily is used to assess the function of the autonomic nervous system (ANS), it consists of the sympathetic nervous system and the parasympathetic nervous system which coordinates the activities of the bodies unconscious actions as a part of the peripheral nervous system. SNS is known as the fight and flight response, it operates within the middle of the spinal cord and activates in response to stress causing an increase in HR, constriction of blood vessels and an increase in blood pressure in order to maintain homeostasis, a healthy/stable state of the body. PNS is known as the rest and digest mechanism, the activities of the PNS contradicts SNS, it relaxes the heart which slows down the heart rate, lowers stress and decreases blood pressure. SNS and PNS work together to maintain a balance, also known as the sympathovagal balance, allowing humans to be safe and sound or an imbalance would indicate abnormalities associated with the heart [1]. In order to accurately assess the function of the ANS, time and frequency domain methods are utilized. Time domain parameters include features such as: a) standard deviation of $\mathrm{NN}$ - intervals $(S D N N)$, b) square root of the mean of the sum of the squares of differences between successive $\mathrm{NN}$ - intervals (RMSSD) and $\mathrm{c})$ proportion of the number of $\mathrm{NN}$-interval difference of successive $\mathrm{NN}$ - interval which are greater than $50 \mathrm{~ms}$ divided by the total number of NN-interval (PNN50) [2]. These methods can efficiently analyze HRV through the analysis of the $R-R$ interval which can indicate changes in 
the HR due to the activities of the SNS or PNS but it's not a sufficient method to discriminate between the SNS and PNS [2]. Frequency domain methods such as $L F(0.04 \mathrm{~Hz}-0.15 \mathrm{~Hz})$ and $H F(0.15 \mathrm{~Hz}-0.4 \mathrm{~Hz}), L F / H F$ ratio are often utilized to differentiate between the activity of the SNS and PNS. $L F$ primarily indicates the activity of the SNS but is also partially associated with the activity of the PNS, while $H F$ indicates the activity of the PNS, and their ratio $L F / H F$ is used to determine the sympathovagal balance [2]. These indices have made it possible to detect many abnormalities, diseases and possible indication of mortality due to the distorted activity of the heart and the peripheral nervous system. HRV has been used for various applications in research studies which includes: analysis of mental and physical stress, classification of drowsiness and other sleep states, analysis of athletic performance which is used to prevent/assess performance fatigue, mental fatigue, how being sedentary affect physical/mental well-being, analysis of meditation, influence of drugs of HRV, analysis of anxiety and depression in females and various other morbidities associated with reduced HRV as well the downfall associated with increased HRV.

(Kim H et al., 2018) presented a review paper to analyze HRV and stress, the study described the physiological function associated with stress, HRV related to specific parts of the brain/heart anatomy responsible for the changes associated with stress [80]. The paper presented information related to the anatomy/physiology behind stress, but neglected wearable devices used for data collection, tools used signal processing, machine learning models used for classification of pathologies, wireless monitoring of HRV to improve the health care system and ultimately patient's health and the various applications associated with HRV research.

This article will analyze the various abnormalities associated with HRV, their detection and analysis using an ECG (electrocardiogram), Respiration, GSR and other wearable devices. The impact of pathologies on the human body and mental state as well as the possible gaps that are associated with each research study.

\subsection{Methods}

The literature survey was performed through Ryerson University Library and Archives online system primarily on PubMed, IEEE Xplore, Web of Science, Scopus and Google Scholar. The 
search was allocated towards HRV studies using ECG, EDA, RESP, PPG signal analysis, few papers involved the analysis of EEG or EOG, but were not considered to present information primarily based on ECG, EDA, RESP, PPG signal analysis. All the reviewed articles were published after 2010 to present information which is not outdated, except one paper which was used to present the function of time and frequency domain analysis. The relevant papers which were reviewed and summarized described the morbid conditions/situation associated with HRV in depth and in detail, any paper which only briefly discussed HRV were not considered. Papers which primarily focused on factors outside of HRV were also not considered. More than 70 papers were reviewed but a lot of them revolved around certain major topics analysed through HRV such as cardiac function, exercise or drowsiness. Accounting for repetitive topics, 18 major concepts were discussed in depth. The gaps associated with each article were acknowledged and presented. Few science-based websites (2) were used to introduce relevant information associated with ANS.

Table 1: Research paper associated with HRV detected using an ECG, type of study, results of $H R V$, concepts being analyzed.

\begin{tabular}{|c|c|c|c|c|c|}
\hline Author & Features & Application & Modality & Notable Results & $\begin{array}{l}\text { Method of } \\
\text { Analysis }\end{array}$ \\
\hline $\begin{array}{l}\text { Rosenberg et al } \\
{[2]}\end{array}$ & $\begin{array}{l}L F n, H F n, L F / H F n \\
L F p, H F p, L F / H F p\end{array}$ & $\begin{array}{l}\text { Stress test in } 1 \mathrm{D} / 2 \mathrm{D} \\
\text { study }\end{array}$ & ECG & $2 \mathrm{D}$ accuracy $90 \%$ & $\begin{array}{l}L F, H F \text { 2D scatter } \\
\text { plot. }\end{array}$ \\
\hline Blood et al. [8] & $\begin{array}{l}L F, H F, V L F, L F / H F \\
\text { ratio }\end{array}$ & HRV due to depression & ECG. & HRV decreases & $\begin{array}{l}\text { Frequency } \\
\text { domain }\end{array}$ \\
\hline Molina et al. [58] & $\begin{array}{l}p N N 50, R M S S D, L F n, \\
H F n, L F / H F,\end{array}$ & $\begin{array}{l}\text { HRV from Supine and } \\
\text { orthostatic position }\end{array}$ & ECG & $\begin{array}{l}\text { HRV reduced post } \\
\text { exercise }\end{array}$ & Time Domain \\
\hline Leti et al. [10] & $\begin{array}{l}R M S S D, p N N 50, L F, \\
H F\end{array}$ & $\begin{array}{l}\text { HRV due to training } \\
\text { and overtraining }\end{array}$ & ECG. & $\begin{array}{l}\text { SNS dominant post } \\
\text { exercise }\end{array}$ & $\begin{array}{l}\text { Time and } \\
\text { Frequency } \\
\text { domain }\end{array}$ \\
\hline Walker et al [12]. & $L F, H F, R M S S D, S D N N$ & HRV due to noise & ECG & $\begin{array}{l}\text { HRV reduced from } \\
\text { noise } \\
\end{array}$ & $\begin{array}{l}\text { Time and } \\
\text { Frequency } \\
\text { domain. }\end{array}$ \\
\hline Wang et al [13]. & $\begin{array}{l}S D N N, L F n u, H F n u \text {, } \\
L F / H F \text { ratio, } R-R \\
\text { interval. }\end{array}$ & HRV due to CHF & ECG & $\begin{array}{l}100 \% \text { classification } \\
\text { accuracy }\end{array}$ & SVM, KNN \\
\hline
\end{tabular}




\begin{tabular}{|c|c|c|c|c|c|}
\hline Chang et al [23]. & $L F$ and $H F$ & $\begin{array}{l}\text { HRV due to anxiety } \\
\text { and depression }\end{array}$ & ECG & HRV decreases & $L F, H F$ \\
\hline Pinheiro et al [14]. & $\begin{array}{l}S D N N, R M S S D, L F, \\
H F, L F / H F \text { ratio. }\end{array}$ & HRV from MI & ECG. & HRV decreases & $\begin{array}{l}\text { Time and } \\
\text { Frequency } \\
\text { domain }\end{array}$ \\
\hline Toni et al [15]. & $L F, H F, L F / H F$ ratio. & $\begin{array}{l}\text { Impact of CVD and } \\
\text { depression on HRV }\end{array}$ & ECG & HRV decreases & $L F / H F, L F, H F$ \\
\hline Shi et al [16]. & $\begin{array}{l}S D N N, R M S S D, \\
P N N 50, L F, H F \text { and } \\
H R .\end{array}$ & $\begin{array}{l}\text { Correlation between } \\
\text { HRV and emotion }\end{array}$ & ECG. & $\begin{array}{l}L F / H F \text { increases due to } \\
\text { happiness }\end{array}$ & $\begin{array}{l}\text { Time and } \\
\text { Frequnecy } \\
\text { domain }\end{array}$ \\
\hline $\begin{array}{l}\text { Ponnusamy et al } \\
\text { [17]. }\end{array}$ & $L F, H F, R M S S D$ & HRV due to seizure & ECG & $\begin{array}{l}L F \text { increases, } \mathrm{HRV} \\
\text { reduced }\end{array}$ & $\begin{array}{l}A p E N, L F, H F \\
R M S S D\end{array}$ \\
\hline $\begin{array}{l}\text { Howells et al } \\
\text { [28]. }\end{array}$ & $H F$. & HRV due to BPD & ECG & HRV reduced & $H F$ \\
\hline $\begin{array}{l}\text { Rios-Aguilar et al } \\
{[30] .}\end{array}$ & $\begin{array}{l}\text { R-R interval RMSSD, } \\
S D N N\end{array}$ & HRV due to drowsiness & $\begin{array}{l}\text { ECG } \\
\text { smartwatch. }\end{array}$ & HRV increases & Time Domain \\
\hline Jung et al [36]. & $\begin{array}{l}L F, H F, R M S S D \\
L F / H F\end{array}$ & HRV from fatigue & ECG sensor & HRV decreases. & $L F / H F, R M S S D$ \\
\hline Rahim et al [39]. & $L F / H F, L F, H F$ & HRV and drowsiness & ECG, PPG & $H R$ decreases & $L F / H F$ \\
\hline $\begin{array}{l}\text { Purnamasari et al } \\
\text { [45]. }\end{array}$ & $\begin{array}{l}R M S S D, S D N N, R-R, \\
L F / H F\end{array}$ & HRV from drowsiness & $\begin{array}{l}\text { Bioradio } \\
\text { wireless ECG. }\end{array}$ & $L F / H F$ decreases & $\begin{array}{l}\text { RMSSD, SDNN, } \\
L F / H F\end{array}$ \\
\hline $\begin{array}{l}\text { Georgiou et al } \\
{[37] \text {. }}\end{array}$ & $\begin{array}{l}S D N N, p N N 50, R M S S D \\
L F, H F, L F / H F\end{array}$ & $\begin{array}{l}\text { HRV during physical } \\
\text { exercise }\end{array}$ & ECG, PPG & $\begin{array}{l}91-99 \% \text { accuracy in } \\
\text { time domain }\end{array}$ & $\begin{array}{l}\text { Time and } \\
\text { Freqeuncy } \\
\text { domain }\end{array}$ \\
\hline Gontier et al [47]. & $\begin{array}{l}R M S S D, L F / H F L F, \\
H F .\end{array}$ & $\begin{array}{l}\text { HRV from mind } \\
\text { wandering }\end{array}$ & ECG & $\begin{array}{l}\text { Lower } L F, H R \text { during } \\
\text { low attention }\end{array}$ & $L F / H F, R M S S D$ \\
\hline $\begin{array}{l}\text { Sahayadhas et al } \\
\text { [48]. }\end{array}$ & $L F, H F, L F / H F$ & $\begin{array}{l}\text { HRV due to } \\
\text { drowsiness }\end{array}$ & ECG, EMG & $L F / H F$ decreased & $\begin{array}{l}\text { Frequency } \\
\text { Domain }\end{array}$ \\
\hline Vicente.J et al [3]. & $L F p, H F p, L F / H F, T P$ & HRV and Drowsiness. & ECG & $\begin{array}{l}\text { PPD } 96 \% \text {, sensitivity } \\
59 \% \text {, specificity } 98 \%\end{array}$ & $\begin{array}{l}\text { Frequency } \\
\text { Domain, LDA. }\end{array}$ \\
\hline $\begin{array}{l}\text { Posada-Quintero } \\
\text { et al. [67] }\end{array}$ & $S C L, S C R, H F, L F, L F n$ & $\begin{array}{l}\text { Attention due to lack of } \\
\text { sleep }\end{array}$ & ECG, GSR & $\begin{array}{l}\text { Accuracy of } 66 \%, 66 \% \text {, } \\
62 \%\end{array}$ & KNN, SVM, LDA \\
\hline He et al. [70] & $\begin{array}{l}S D N N, S D 1, S D 2, \\
A p E N, L F, H F, L F / H F\end{array}$ & HRV due to stress & ECG & $\begin{array}{l}\mathrm{CNN} \text { had } 17.3 \% \text { error } \\
\text { rate }\end{array}$ & CNN, SVM, LDA \\
\hline
\end{tabular}




\begin{tabular}{|l|l|l|l|l|l|}
\hline $\begin{array}{l}\text { Pathoumvanh et } \\
\text { al. [115] }\end{array}$ & Single beat ECG feature & HRV due to jogging & ECG & $\begin{array}{l}\text { 97\% classification } \\
\text { accuracy }\end{array}$ & LDA \\
\hline Schmidt et al[60] & $L F, H F, L F / H F, S T$ & HRV due to stress & $\begin{array}{l}\text { ECG, GSR, } \\
\text { RESP }\end{array}$ & $\begin{array}{l}\text { 93\% accuracy (binary), } \\
80 \% \text { (multilevel) }\end{array}$ & $\begin{array}{l}\text { Adaboost, LDA, } \\
\text { DT, RF, KNN }\end{array}$ \\
\hline Cho et al. [61] & $\begin{array}{l}L F, H F, L F / H F, S C R, \\
S C L, S T\end{array}$ & HRV due to stress & $\begin{array}{l}\text { GSR, ECG, } \\
\text { PPG }\end{array}$ & $\begin{array}{l}95 \% \text { classification } \\
\text { accuracy }\end{array}$ & K-ELM NN \\
\hline
\end{tabular}

Table 1 describes the various HRV research paper's reviewed, the papers were obtained from search engine such as RULA, Google Scholar, NCBI, IEEE.

The research paper published by Rosenberg et al [2] revealed that the accuracy of 1D parameters (LF/HF linear relationship reveals information about HRV) studies involving stress was around $70 \%$, being as high as $89 \%$ when analyzing $L F p$ and $L F i A$ during an emergency situation or as low as $37.1 \%$ with regards to the calculation of $L F p$ during a pain period. $2 \mathrm{D}$ scatter plot were a more efficient method of analysis with a classification accuracy revolving around $90 \%$ when assessing stress in different situations, it classifies 2DiA (instantaneous amplitude) during a conference, mental stress test, emergency with 100\% accuracy and dropped as low as $66.3 \%$ during the $2 \mathrm{D}_{\mathrm{n}}$ (normalized) analysis of pain [2].

HRV is much harder to detect in motion, sensitivity of drowsiness/awake detection was 0.59 and 0.62 using a drowsiness episodes detector and a sleep deprivation detector respectively during real/virtual driving simulations as revealed by Vicente et al [3]. The specificity and positive predictive value were much more efficient, which was 0.98 and 0.96 using a drowsiness episodes detector, 0.88 and 0.80 using a sleep deprivation detector [3].

$L F / H F$ represents the sympathovagal balance, which is proportional to $\mathrm{HRV}$, decreased ratio is proportional to an increase in HRV vice versa an increase in ratio corresponds to a decrease in HRV. Increased HRV is associated with increased PNS activity, which is generally associated with higher values of RMSSD, SDNN, PNN50 in time domain and increased $H F$ in frequency domain. Decrease in HRV is associated with increased SNS activity, increased $L F$ in frequency domain and lower values from $R M S S D, P N N 50, S D N N$ in time domain, although time domain variables are not linear with HRV or as accurate in terms of measuring HRV, some variables 
such as $S D N N$, RMSSD may result in low values in response to an increase in HRV, the values obtained in time domain varies and the correlation with HRV is not as coherent as frequency domain variables. The most efficient window to measure HRV in time or frequency domain is 25 min epoch (period) with 10-30 seconds intervals (RMSSD, PNN50 require ultra-short intervals for an efficient computation) [2]. Increased HRV can keep up better with changes due to the activity of the heart, represents a relaxed state when a person is resting, and is associated with maintaining homeostasis (balance represents good health), it also represents a transition from awake to sleep which can cause accidents on the road due to drowsiness which is a worst scenario regarding any health concerns associated with increased HRV. Decreased HRV is associated with various cardiovascular diseases such as myocardial infarction (heart attack), hypertension (high blood pressure), depression, anxiety, increased HR, epilepsy, insomnia, pain, fatigue and stress.

Computation of HRV often requires an ECG, PPG can be a good alternative due to its mobility, simplicity, time efficiency and easy operability [4], wearable devices (ECG, PPG) are more mobile and ambulatory, recent android development are integrating apps which can process wearable devices in order to assess a patient's HRV, making it easier to diagnose patients who suffered from myocardial infarction, hypertension while they live their daily life.

HRV has a wide range of applications, some of those applications were presented in Table $\mathbf{1 .}$

Computation of HRV often requires an ECG, PPG can be a good alternative due to its mobility, simplicity, time efficiency and easy operability [4], wearable devices (ECG, PPG) are more mobile and ambulatory, recent android development are integrating apps which can process wearable devices in order to assess a patient's HRV, making it easier to diagnose patients who suffered from myocardial infarction, hypertension while they live their daily life.

\subsection{Organization of the Thesis}

This thesis explores the various cardiac morbidity associated with impaired HRV, which develops from stress. It investigates the feature variation (obtained from ECG, GSR and Respiration) associated with reduced or healthy HRV, due to 4 distinct phases which are 
mentioned below. Machine learning models are utilized to classify stress from HRV features. Chronic stress can lead to various cardiac morbidity and is a major cause of mortality for patients suffering from hypertension and myocardial infarction. This study examines various physiological signals which can be utilized to detect/analyze stress, and accurately classify stress through machine learning algorithms, from VR roller coaster simulation and VR fishing video game. Thus, allowing us to understand the impact of effective emotion, perception, VR simulation and relaxing video games on stress induction and reduction.

\section{Chapter 2: Literature Review}

This chapter presents the literature review, over 50 research papers were utilized to understand the severe impact of impaired HRV, which leads to various cardiac morbidities and is a major cause of cardiac mortality. It also investigates wearable devices utilized to detect physiological signals, from which specific features are extracted to analyze abnormal and healthy HRV. Presents certain methods which can be utilized to detect and classify abnormal HRV remotely. The rest of the chapter discusses about the various research conducted to understand the development and effect of stress, especially from VR simulations.

\section{Chapter 3: Physiological Signal Analysis of Stress}

This chapter presents a thorough analysis of 3 physiological signals (ECG, GSR, RESP) obtained from 4 distinct phases which are: (1) a baseline phase which was used to assess the subjects normal physiological function, (2) a virtual reality roller coaster phase which was meant to induce stress through the elicitation of affective emotion such as fear, which is perceived as a threat, (3) a cognitive color stroop test was also used to induce stress and increase anxiety, and (4) a VR fish game was required to understand the impact of playing video games to reduce stress and anxiety.

\section{Chapter 4: Machine Learning}

This chapter delves into the use of machine learning models to classify stress. Model evaluation through confusion matrix, precision recall curves and ROC curves. Discusses the effectiveness of certain models and deficiency of others, with regards to stress classification. 


\section{Chapter 5: Conclusion}

This chapter presents a summary of the discussion, interpretation of the results, limitations of the experiments conducted and future possibilities.

\section{Chapter 1- Introduction}

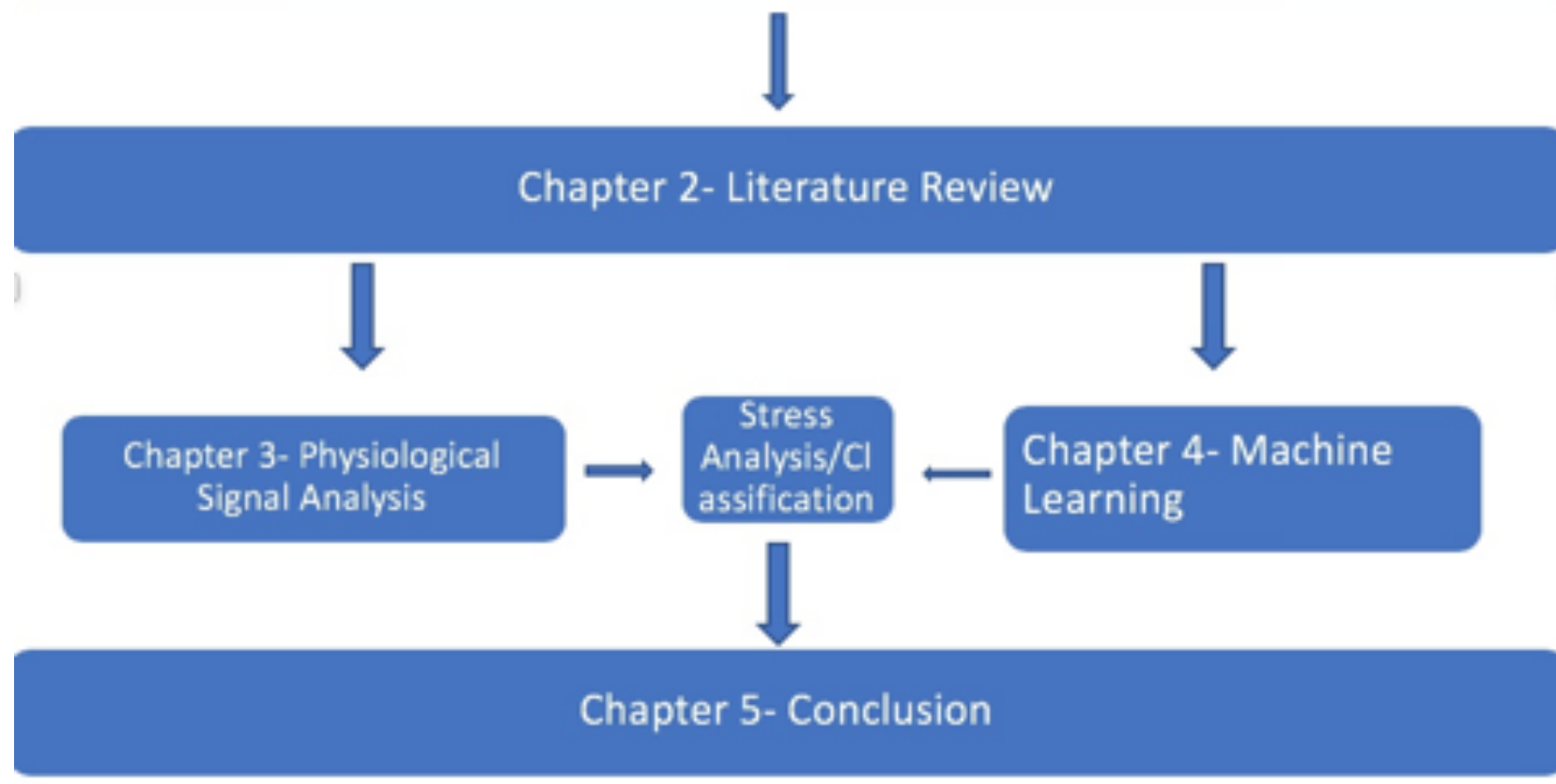

Figure 2: Block diagram which indicates the organization of the Thesis. 


\section{Chapter 2}

\section{Literature Review}

\section{Overview of Methodologies}

\subsection{Data Collection}

Smartphones used to assess HRV/stress

Recent smartphones are more than just a device used for communication and listening to music, these devices include embedded sensors, accelerometers, microphones, digital camera, and various apps based on measuring the affective state (neural, emotion, stress) of an individual which can be utilized to monitor physiological activity, position, speech patterns, facial expression and affective state which gives them the ability to analyze their stress level, behavior and emotion at anytime and anywhere promoting better human health and well-being [63][64]. (Muaremi et al., 2013) utilized a smartphone along with wearable devices in order to assess stress during the workday and sleep. Prolonged work period without sufficient rest/recovery periods can reduce happiness and lead to chronic stress due to mental workload [63]. Smartphones were used to collect audio, communication and physical activity data during work periods and wearable Wooho chest belt was used to collect HRV data during sleep [63]. These developments allowed for the analysis of speech, communication, accelerometer (position), and HRV resulting in data that can detect the level of stress associated with various activities such as communication, sleep and work [63]. (Kanjo et al., 2015) revealed that stress and negative emotions are associated with certain pitch (speech), expressive facial expression (tired/angry from stress), decreased HRV and physiological activity, which can be monitored throughout the day and during sleep using a smartphone and wearable device allowing people to detect irregularities before it becomes chronic and fatal as well as predict the outcome due to certain activities which should be minimized to increase the level of productivity and well-being. EDA (that measures GSR and skin temperature), ECG (measures HR, HRV) are the most effective devices to detect stress especially in motion, frequency domain features such as $L F, H F$ and MeanNN, stdHR time domain features can be utilized to classify stress using multinomial logistic regression model, kNN and SVM [64]. Stress is not a very complex activity to detect or analyze, (Muaremi et al., 2013) were able to produce 93.6\% accuracy through binary (stress, no stress) 
classification of stress using multinomial logistic regression model but it is quite complex to distinguish between low, mid and high level of stress, as indicated by a low classification accuracy of $61 \%$ [64]. (Schmidt, et al., 2018) produced classification accuracy of $72 \%$ upon analyzing high, mid and low level of stress with RF (linear regression $=70 \%$ accuracy), further demonstrating that chronic stress is hard to predict, although stress can be distinguished from a relaxed state with high efficiency [64]. The best solution with regards to chronic stress is preventing it through a healthy lifestyle, adequate rest, positive behavior/emotion and good time management which can reduce stress and improve health.

\subsection{Feature Extraction}

HRV processing method

HRV is hard to detect simply because in order to accurately measure the rate of change associated with $R-R$ interval obtained from the QRS complex, the raw ECG signal needs to be filtered and processed. Raw ECG signal can be obtained through an analog ECG circuit with electrodes attached to the limbs. Recently wearable ECG and PPG devices have been developed

which can be used to detect ECG signal wirelessly. Raw ECG signal needs to be filtered using a band pass filter which can filter the low and high frequency noise in the range of 0.5-35 $\mathrm{Hz}$ (just an example range) [56]. After filtering, the ECG signal is a lot smoother and cleaner which makes it easier to detect the QRS complex. Time domain parameters can be obtained from the QRS complex but in order to secure frequency domain parameters, spectral transformation of the QRS complex is required through PSD (power spectral density), which can be obtained through FFT (represents frequency components), AR (reduces spectral leakages to improve the resolution of the data) analysis of the QRS complex [56]. Time domain parameters are statistical evaluations of the ECG signal (presents statistical properties) and frequency domain parameters describes how power (variance) is dispersed as a function of frequency [55].

\subsection{Wearable Devices}

Wearable Devices used to Detect Stress

Stress is associated with the function of the ANS which is most commonly derived from HRV. There are various wearable devices which can be used to assess the rate of change associated 
with autonomic activity, few of those devices are displayed below in Figure 3. ECG is the most commonly used device with respect to HRV detection [2][4][59][60]. (Rosenberg et al., 2017) utilized a wireless ECG sensor during various situations to measure stress response associated with a particular event, (Schmidt, et al., 2018) developed WESAD, a public database to analyse affective state by utilizing Emphatica E4 which measures BVP, EDA, ACC and TEMP and RespiBAN which can detect respiration and ACC (accelerometer) [60]. (Cho et al., 2017) analyzed HRV, skin conductance/sweat and skin temperature through PPG, EDA and SKT respectively. (Georgiou et al., 2018) revealed that wearable devices can detect HRV at rest with 85\% accuracy using PPG and 99\% accuracy using ECG which deteriorates to 85\% accuracy during exercise [4]. Ambulatory detection of HRV is the current resolve for most researchers who hope to make a pragmatic and positive impact on the health and well-being of patients suffering from CVD, hypertension, diabetes, chronic stress, myocardial infarction and as a result they need to be monitored throughout the day to prevent a serious calamity, but cannot make the effort to visit the hospital all the time due to the considerable distance and lack of physical ability. (Cho et al, 2017) was able to classify stress with $95 \%$ accuracy which indicates that wearable devices along with ML can be utilized to efficiently assess HRV which would allow physicians to predict certain outcomes and prevent common tragic events associated with CVD such as heart attack, stroke and especially death. Although the expense, adaptability and functionality are still a major concern with wearable devices, especially if they were to be integrated with ML which poses as a major setback, and might be the reason that prevents the development of such devices. 


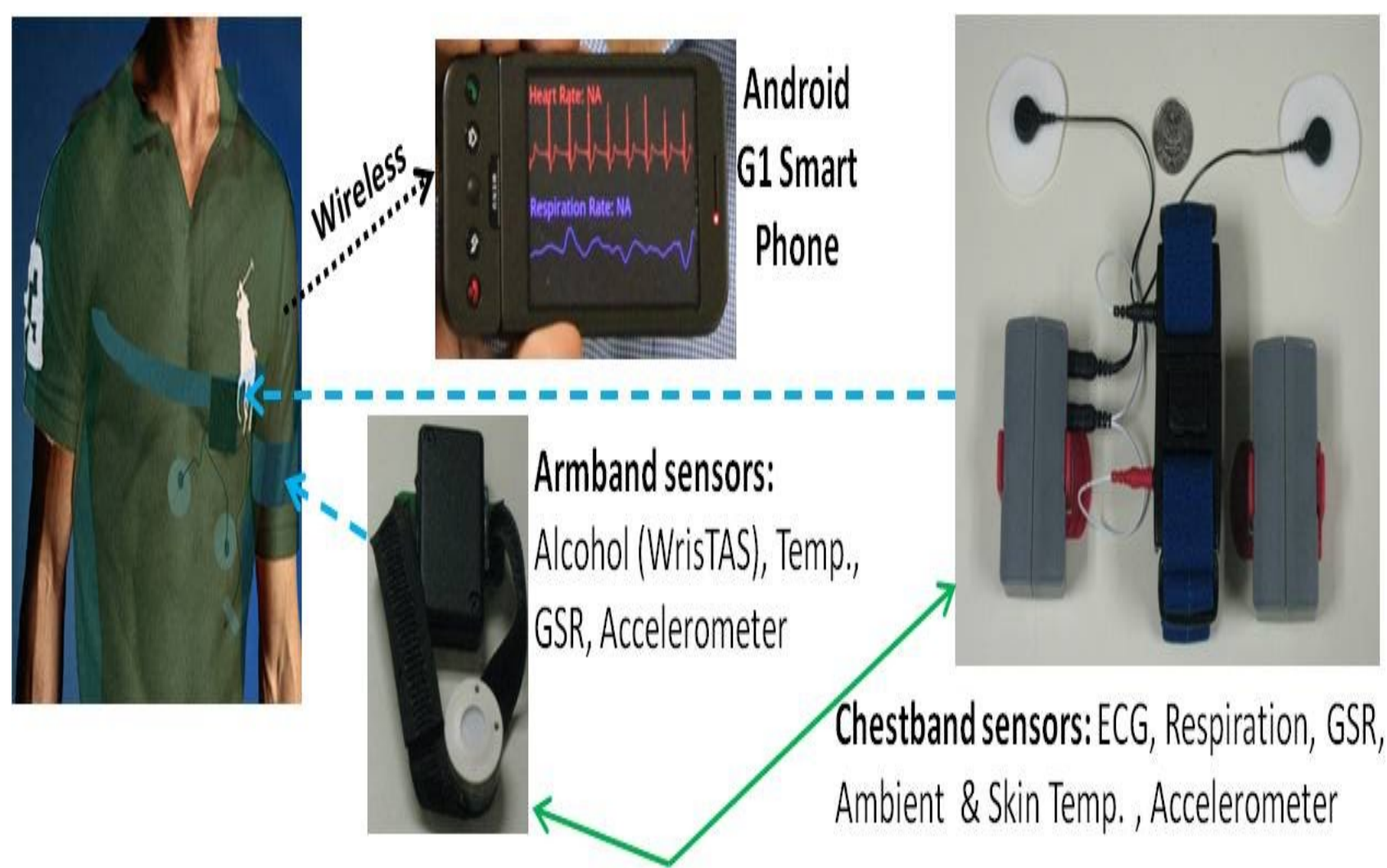

Figure 3: Wireless devices used to detect ECG, Respiration, GSR, skin temperature [72].

\subsection{Machine Learning}

2.4a Convolutional Neural Network (CNN) used to detect stress through HRV

Stress is associated with several mental and health problems making it crucial to manage stress through established methods [70]. 77\% of the US adult population suffered from physical symptoms and $73 \%$ suffered from psychological symptoms due to stress [70]. In US \$300 billion are lost due to missed work as a result of stress, UK lost 13 million working days in a year (people) which resulted in losing 12 billion pounds [70]. Stress results in hyper-activation of SNS and suppression of PNS [70]. CNN utilize automatic feature learning; it is utilized in this paper for accurate and fast analysis of cognitive stress through HRV features [70]. $6 \mathrm{HRV}$ methods were compared with CNN method for detecting cognitive stress [70]. CNN is an ANN which contains convolutional layer in its hidden layer, CNN efficiently learns the features of the given task, It could capture relevant information automatically from the input [70]. Mental calculation was performed to induce acute cognitive stress, muscle contraction and respiration noise was removed through 1-50 Hz Bandpass filter (ECG), 10 second window was utilized for 
feature extraction. Spectrum derived from R-peak positions were used as for CNN to reduce noise associated, Lomb scargle was used to obtain PSD, band from 0.04- $20 \mathrm{~Hz}$ was adapted as an input for CNN [70]. CNN with 10 layers were used for this study, first layer was an image of the input layer, size 799x1x1 = input frequency, second layer was a CNN which consisted of 6 filters, 4x1x1 with stride 1-by-1 (filter over 1 filter to the next) [70]. Followed by batch normalization layer (normalization, higher learning rates, reduced overfitting, allows each layer to learn independently, makes sure there is no activation that is too high or low, allow for training things we couldn't train, increase stability of NN, dropout certain units to reduce overfitting), RELU layer (sum of weighted connection between two nodes) input which transformed into output, output is zero if input is negative, or output is input directly if it is positive), and a dropout layer (drop units (hidden layers) to reduce overfitting) with a probability of 0.5 [70]. Figure 4 displayed below describes the activation layers associated with stress. The next three layers were two fully connected layers with batch normalization between them, the last two layers are SoftMax (takes input and transforms into probability in the last layer) layer and classification output layer. Stochastic gradient descent with momentum (SGDM) optimizer was used to train CNN [70]. Maximum number of epochs was 30, learning rate started at 0.001, reduced by a factor 0.1 every 10 epochs [70]. 4-fold cross validation was used to test the model accuracy, it was also used to test the ability of model prediction in machine learning [70]. Data was divided into 4 partitions, 3 for training, 1 for testing, 1278 training samples and 426 testing samples an average of the four-fold cross validation indicated model performance [70]. SDNN, $S D 1, S D 2, S D 1 / S D 2(p Q), L F, H F, L F / H F . S D N N, p Q, S D 2$ features were recorded using ultra short windows (1 min) [70]. Extracted feature (CNN) fed into the SVM and LDA for classification, split into 75\%, 25\% training, test data and data was processed in MATLAB [70]. Error rate false acceptance rate (FAR), false rejection rate (FRR) were used for performance evaluation, ANOVA was also used to assess detection performance [70]. CNN had 17.3\% error rate which was $7.2 \%$ lower than and $32.6 \%$ lower than comB (combination of feature) with SVM and $L F / H F$ ratio with SVM respectively [70]. CNN better than conventional method in terms of $E R$ and FAR (false acceptance rate) [70]. The output of stress and rest were normalized and averaged across all subjects, with regards to the last 4 layers, cubic interpolation was used to resize the length of each feature map, fit dimension of the first layer, image input [70]. ER (error) decreased with increase in frequency range from 0.4-6 [70]. CNN information on stress was 
obtained through the activation difference between stress and rest state of the first 5-layer, classification performance due to various inputs with different frequency bands was calculated [70]. Main information with regards to stress detection is below $2 \mathrm{~Hz}$ [70], bias is a constant used to improve output, reduced bias through relaxation (listening to music) increased reliability of the data, 0.4-2 were able to discriminate between stress and rest [70]. CNN had a $17.3 \%$ error rate using a super short time window of $10 \mathrm{~s}$ and it performed better than other established HRV methods, CNN resulted in less false stress detection [70]. The overall accuracy obtained using CNN was not mentioned, error rate does not indicate the efficiency of classifying stress leading to the assumption that the classification accuracy requires further improvement and it might be less than $70 \%$ which would not result in a valid prediction which can be used to accurately antedate stress within subject with a disease such as myocardial infarction or hypertension (stress is fatal for patients suffering from myocardial infarction).

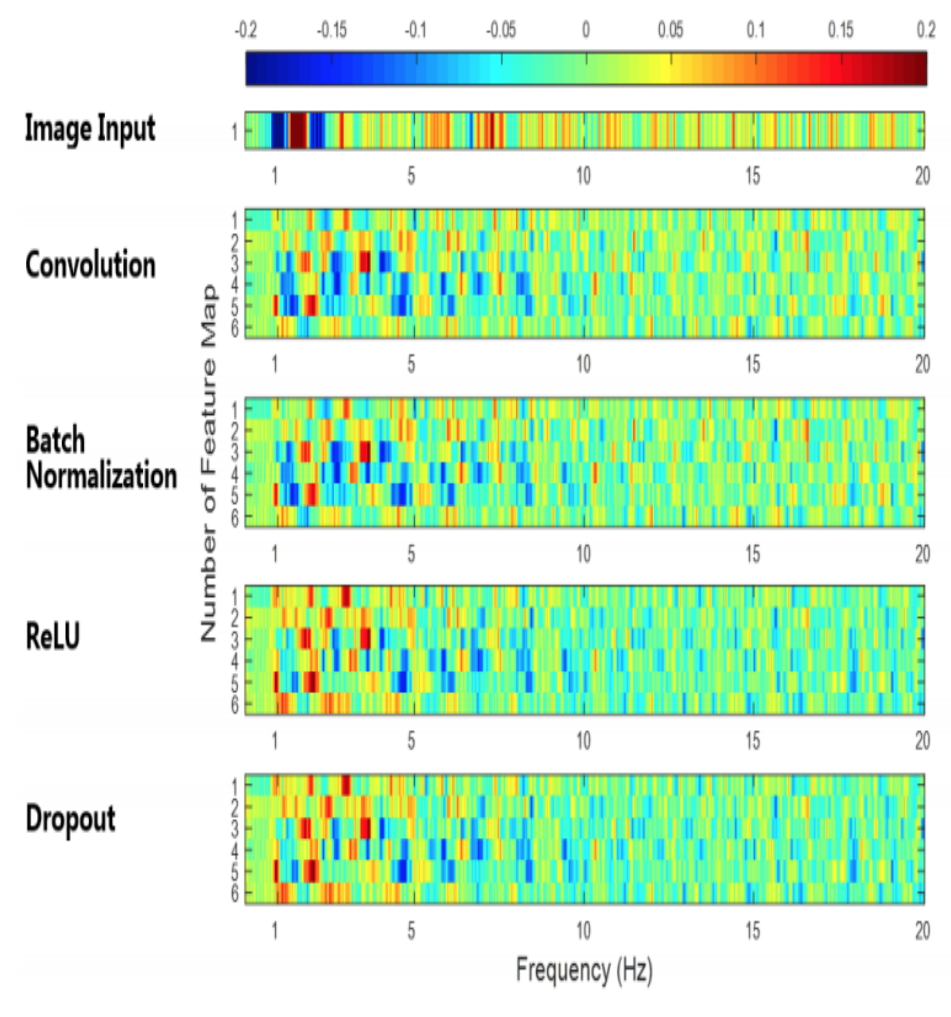

Activation Difference of Each Layer between Rest and Stress
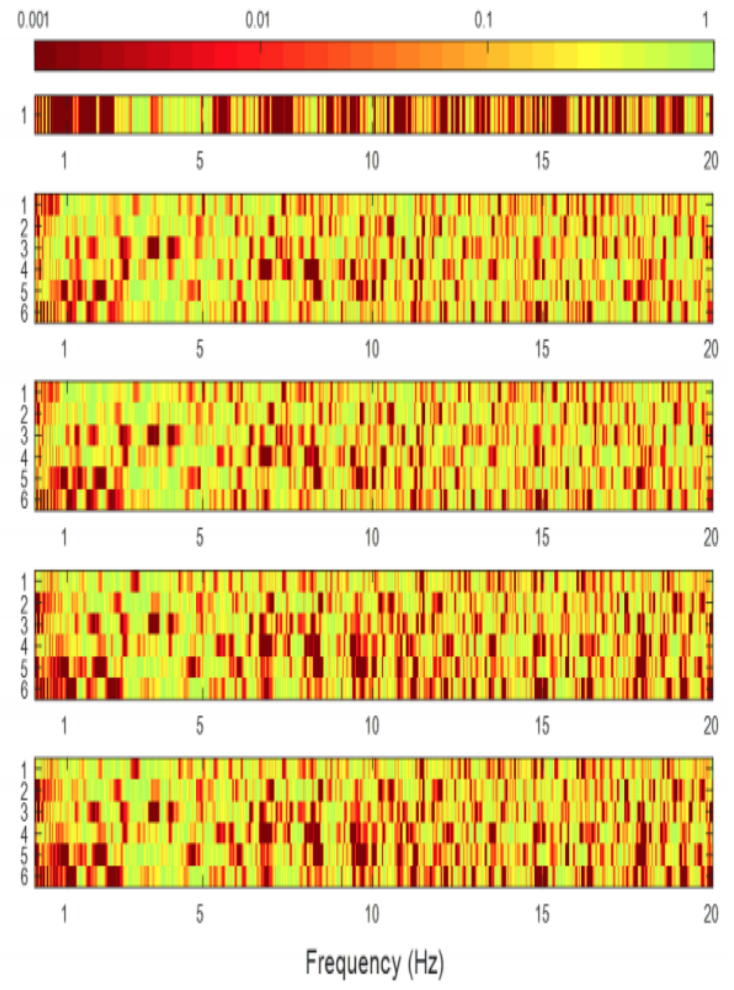

$p$ Value of Activation Difference

Figure 4: Activation difference for first five CNN layer between rest and stress state [70]. Pending permission for reproducing this figure 


\section{4b Ultra Short periods to assess HRV/stress}

Stress is most commonly measured through HRV, indicated by the increase in SNS activity of the ANS which decreases HRV. (Rosenberg et al., 2017) have indicated that time of the epoch used to assess HRV in time domain is very important, $3 \mathrm{~min}$ is the minimum epoch that can be used by RMSSD in order to measure fatigue in athletes, but 5 min epoch are optimal for stress analysis otherwise preprocessing the signal may lead to filtering out most of the data which would result in inadequate and less efficient output and representation of HRV activity associated with stress [2]. (Castaldo et al.,2019) analyzed HRV using ultra-short term HRV features in order to assess mental stress [62]. Theoretically stress is generally associated with perception, it can be due to internal perception such as negative emotions of anger, anxiety, fear, depression and mood swings or it may be induced by external perception of the world around us, such as an upcoming exam, presentation or deadline which causes us to worry, lose sleep and accumulate stress [59]. ECG, GSR, HRV, EEG and respiration rate were used to analyze/detect stress, Mean NN, stdNN, meanHR, stdHR, HF, SD2 are utilized as ultra-short features which can be utilized to assess HRV within 3 min allowing us to understand the level of stress associated [62]. MeanNN, stdHR, $H F$ features resulted in the best accuracy when classified through an automated classifier such as TPOT which classifies HRV using various ML models (SVP, MLP, neighbour search IBK, C4.5 and LDA) and indicates the level of accuracy associated with each model [62]. ANOVA statistical analysis method was used to determine/distinguish the best features to discriminate between rest and stress state [62]. Machine learning is a powerful tool which is currently prevalent, various models and automatic classifier has allowed for the analysis of HRV and stress through ultra-short term epoch of $3 \mathrm{~min}$ and in certain cases even $1 \mathrm{~min}$ epoch can produce results which can be analyzed to classify stress using specific features, most features are repetitive and has a high correlation with other ones, by reducing such features stress can be classified quickly and with higher accuracy [62].

\section{4c. Classifying HRV from ECG and EDA features through ML classification algorithm} (Posada-Quintero et al., 2019) conducted a study to assess Psychomotor vigilance (PVTmeasures reaction time), auditory working memory (n-back task), visual search (ship task) through ECG, EDA physiological activity and ML classification algorithms [67]. Lack of sleep due to stress reduces vigilance and the ability of working memory regresses with prolonged lack 
of sleep [67]. HP 78354A monitor was used to collect ECG data, GSR amplifier FE116 was used to collect EDA data [67]. PVT required clicking the left button of the mouse as soon as they saw a number (randomly at 2-10s interval) on the screen, which assessed their rate of reaction. In order to test for auditory working memory, the subjects had to indicate whether various tones played progressively were the same as a previously played tune using predetermined keys, positive key indicates identical tone and negative key indicates that they were different. Ship search required the subjects to identify the ship on the computer screen when it appeared, which also analyzed their level of vigilance [67]. EDA was processed in time domain to obtain SCL (low frequency shifts) and SCR (rapid phase shift) [67]. SCL exhibit's the difference between PVT and baseline [67]. SCL is the tonic component, which was significantly different between all three tasks and it indicates the level of attention and reactiveness as well [67]. SCR, $H F, L F$, $H F n$ was significantly different between n-back (working memory task) and PVT [67]. The detection of the activities indicated that PVT, auditory working memory, ship search all had different effect on ANS [67]. SCL, TVsym and LFn which are SNS biomarkers were the most significant differences associated with EDA, ECG activity during each task [67]. Data was classified using Linear kNN, linear SVM, LDA with 66\%, 66\% and 62\% classification accuracies, PVT along with ship search was classified with $69 \%$ classification accuracy using KNN, while working memory was classified with 69\% accuracy using LSVM [67]. The study

was conducted upto 24 hours, classification after 20 hours indicates that ANS activity diminished after 20 hours of wakefulness, but surprising recovered after 24 hours [67]. The low classification accuracy does suggest that the procedure to collect data during PVT, working auditory memory and ship search can be improved, data wrangling process was not efficient, but machine learning classification can be made better with less features if some features include a lot of irrelevant or null values, seaborn is a great way to check for null values using sum and null variables (already exists, just need to import them), and the experiment did not emphasize how lack of vigilance/sleep negatively impact our health such as through stress, drowsiness (leading to car accidents), increased fatigue and headaches [67].

\section{4d. Abnormal HRV classified remotely}

The ANS functions unconsciously and regulates the function of the heart as well as other internal organs such as lungs, kidneys, bladder, digestive and salivary glands. Adults today are more 
prone to stress due to their family and work responsibilities, are unsatisfied in life and at work, lack of sleep due to constantly worrying, less than 5\% find the time for 30 min of physical activity, they typically eat more fast food and less fruits/vegetables or nutrition rich foods which increases blood pressure, reduces good cholesterol while increasing the bad, increases blood sugar level, increases weight leading to pathologies such as hypercholesterolemia, hypertension, diabetes and obesity. Pathologies activates the primary function of the ANS 'flight and fight response' which is regulated by the hypothalamus (located in the CNS) to maintain stable state of the body/recover stable state after being infected by a pathology. Myocardial ischemia (results from reduced blood flow to the myocardium), cardiac arrhythmia is associated with the impairment of the ANS due to hypertension, hypercholerolemia, obesity, diabetes and can lead to chest pain and unfortunately death due to myocardial infarction (cardiac arrest, sudden cardiac death) [65]. One of the key concerns with regards to myocardial infarction is the constant monitoring required to assure the safety of patients who are diagnosed with myocardial infarction, especially those who have to travel a considerable distance to meet with their physician, an ongoing problem for adults who have to work and be responsible for a patient. (Venkatesan et al., 2018) utilized discrete wavelet transform to preprocess ECG signal obtained from a wearable device to extract HRV features, and applied machine learning algorithm to classify cardiac arrhythmias [65]. Preprocessing required an adaptive filter to remove baseline wander (low frequency, non-linear, non-stationary noise), motion artifacts (patient movements which effect electrodes placement), and other interruptions associated with electrode placement and the experiment [65]. Eight time domain features were extracted: RRmean, RRstd(ms), HRV mean, HRstd, RMSSD(ms), NN50, PNN50, RR triangular index, along with 6 frequency domain features: $\mathrm{V} L F(\mathrm{~ms} 2), L F$ power, $H F$ power, $L F \operatorname{norm}(L F / T P-\mathrm{V} L F) * 100, H F$ norm $(H F / T P$ $\mathrm{V} L F) * 100, L F / H F$ ratio which was used to indicate whether the signal detected was normal or abnormal (cardiac rhythm) [65]. Certain features obtained from ECG, blood volume, and HRV parameters were used to train and test the machine learning models specifically SVM classifier which performed better than other models and classified normal/abnormal cardiac arrhythmia with $96 \%$ accuracy [65]. Remote monitoring gives access to patient data and its analysis from a distance, reducing the risk of patients suffering from stroke and increasing the ability to get routine feedback on their health to assure their safety and well-being. Figure 5 displayed below describes the process behind remote analysis of HRV. 

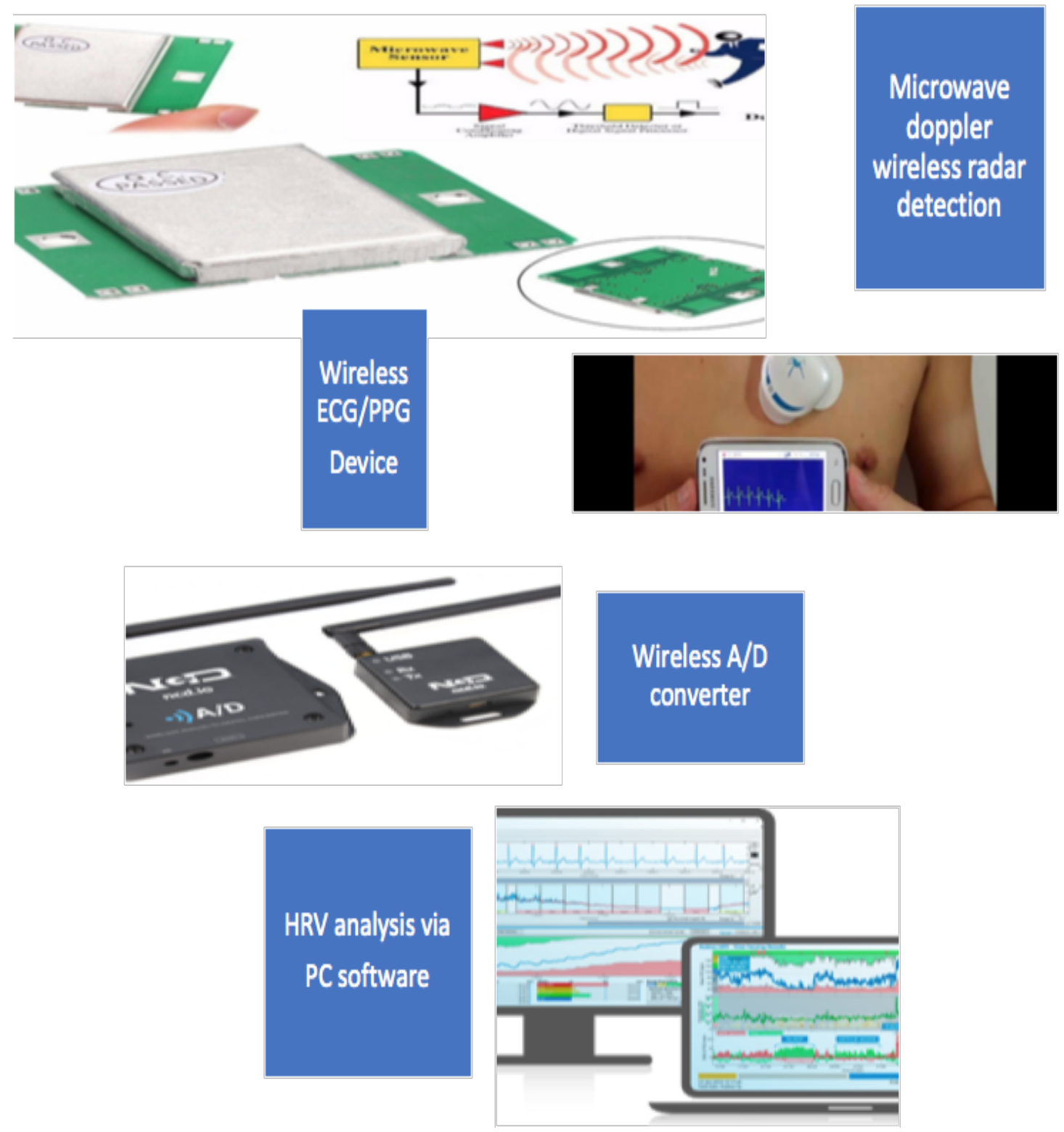

Figure 5: Telemonitoring of HRV through microwave doppler wireless radar [77], wireless ECG/PPG device [78], wireless A/D converter [79], HRV analysis software and internet.

\section{4e. HRV associated with affective computing, classified through NN and SVM}

Wearable devices are becoming more reliable and accurate, research studies are recurrently using wireless ECG and EDA to track CVD, stress, muscle fatigue, cognitive function/ decline due to lack of energy/sleep, indicate whether a person is alive or dead, and detect whether he is suffering from various pathologies (such as hypertension, diabetes, myocardial infarction). 
Mobile devices which can monitor health accurately benefits a high population of people, especially those who are physically incapable of traveling to far distances or don't have the luxury due to time and work. This research is targeting more than just CVD and stress, it is expanding to cancer detection, muscle injuries, circadian rhythm and even the impact of age/gender on affective computing (emotion, stress due to age and gender) . (Rukavina et al., 2016) analyzed physiological signals obtained through EMG, EDA, ECG and respiration to distinguish between various affective states based on gender and age [66]. NN and SVM reported the highest classification accuracy using features Mean, Std, fEMG, low valence low arousal (LVLA), low valence high arousal (LVHA), high valence low arousal (HVLA), high valence high arousal (HVHA), and neutral [66]. Mean, std of GSR detect skin conductance associated with SNS activity, valence, and arousal state detect neural/baseline state which changes due to emotion, $f E M G$ is an effective method to detect pain [66]. Classification was performed through leave one subject out batch validation, the low features and data limits the classification which can be improved through more trials and additional features [66].

(Pathoumvanh et al, 2014) revealed that ECG biometric are different from affective states, they were able to classify HRV conditions with $97 \%$ classification accuracy and $80 \%$ robustness study accuracy using only a single beat ECG feature [66]. Feature extraction was analyzed via continuous wavelet transform and fisher's linear discriminant analysis was used to reduce RMS dimensions [66]. The study did not have a specific purpose outside of HRV detection, stress and pathologies are more complex and much harder to predict using one feature but it might be possible to predict the latter using HRV, the study also did not indicate efficiency of HRV results through any data and such a clever study should aim to be an important factor in the field of medicine and try to assess CVD with high accuracy [66].

\section{4f. Stress Classification through HRV analysis}

Whether it involves an upcoming exam, a big presentation for a conference or a job interview for your dream job, stress is associated with our everyday life. (Abdi, et al., 2018) has indicated that in fact people need a little bit of stress in their life to stay focused, alert and energetic so that they can solve the problems they face in their daily life [59]. Abdi, et al. (2018) also revealed that if people let stress linger around and continue to worry letting it evolve into chronic stress, it can lead to more anxiety, result in a lack of coordination, reduce their level of productivity and if it's 
not detected early, it often leads to many heart related diseases such as hypertension and cardiovascular diseases (CVD), as well as increasing the chance of an infection and it is a major cause of emotional trauma such as depression [59]. (Schmidt, et al., 2018) created WESAD, a multimodal public dataset which includes data for stress and its effect detected through wearable devices [60]. It detects the affective states of users through Emphatic machines such as RespiBAN and Empatica E4 which is placed on their chest and wrist respectively to assess their neural state (baseline brain activity), stress levels and amusement condition (emotional state, in this scenario humor was induced) [60]. Utilizing machine learning classification algorithm such as DT, RF, AB, LDA, KNN, they were able to classify stress/no stress conditions with $93 \%$ accuracy using features obtained from physiological signals (eg: ECG, EDA, Respiration, skin temperature, accelerometer) [60].

Stress is primarily associated with the activity of the SNS, stress hormones are released accordingly in response to certain circumstances, the release of hormones adrenaline and cortisol due to stress enhances alertness and increases muscle stiffness which increases the strength and reaction of the muscle. (Rosenberg, et al., 2017) analyzed the level of stress in response to various situations including public speaking, math, exercise, mediation, pain, cognitive test to induce mental stress as well as the occurrence of stress during an emergency [2]. ECG was used to measure HR, time and frequency domain features such as SDNN, PNN50, RMSSD, LFn, HFn, $L F p, H F p, L F i A, H F i A, L F / H F$ (n,p,IA) were extracted to measure HRV as well as SNS and PNS activity associated with HRV [2]. Time domain results were more indicative of stress and SNS activity instead of raw the ECG signal, frequency domain features were a more accurate representation of $L F, H F$ and $L F / H F$ ratio and $H R V$ especially when it is analyzed with $2 \mathrm{D}$ scatter plots which is more preferable than 1D scatter plots, accuracy of stress detection is around $90-100 \%$ (pain is an exception) using 2D scatter plots which is considerably higher than $70-80 \%$ accuracy achieved using 1D scatter plots [2]. Stress was effectively measured (above $80 \%$ ) in all situations except for pain (around 65\%), doctors have tried to be more detailed to increase the accuracy of detection by using a 10-point pain scale and the results received indicated that pain was too complex to be measured through a simple 10-point pain scale, stress can often cause chest pain, muscle tension, increase the chances of headaches and result in lack of sleep which has yet to be analyzed effectively using wearable ECG devices (EMG is more equipped to 
measure pain) but these symptoms strongly suggest that the subject is suffering from chronic/high level of stress. Wearable devices such as a wearable ECG are a great way to monitor ECG, HR, HRV which are indicative of the level of stress and using established solutions such as meditation can reduce elevated stress levels which hinders the ability to work productively, relax during free time and have a good night of sleep which is essential for living a healthy and productive lifestyle.

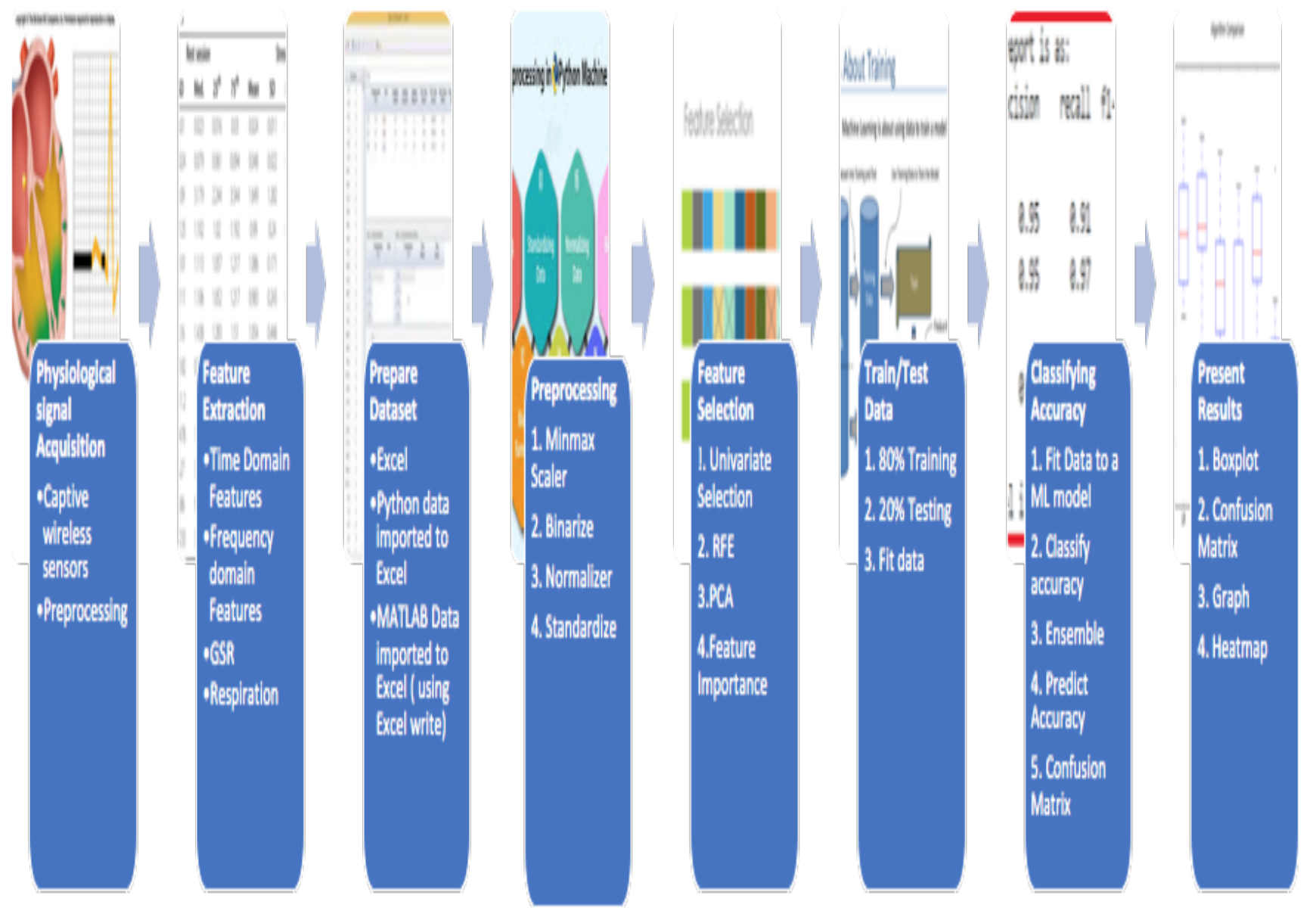

Figure 6: Stress classification through machine learning flowchart.

2.4g. Contradictions associated with stress classification and analysis using features from PPG and EDA signals

Wearable PPG device worn on the wrist and concurrently EDA placed on the fingers allows for continuous monitoring of ANS activity. Ideally, the SNS activity should increase during cardiovascular pathology and mental stress. (Lima et al.,2019) revealed that research experiment 
is sometimes unpredictable as $L F$ and $L F / H F$ activity during stress decreased for certain circumstances where stress was detected. Delineating the changes in ANS activity plays a significant role towards preventing CVD and stress [69]. ANS is regulated by the CNS, it is comprised of multiple neuroanatomical structures [69]. CNS sends ouTPut to SA node in order to adjust to physiological arousal, it's also responsible for responding and adapting to environmental changes and the structures of the brain influences the activity of the heart [69]. Cholinergic fibers are terminal nerve endings associated with PNS which secrete/release acetylcholine which reduces the strength of contraction, which reduces heart rate. Adrenergic fibers are terminal nerve endings associated with SNS activity in order to release epinephrine resulting in increased contraction and HR [69]. EDA signal assess the activity of eccrine sweat gland which is primarily associated with emotional response due to external stimulus and reflects the SNS activity only, since there is no innervation of PNS within the glands. GSR activity represents tonic (skin conductance level - SCL) and phasic component (skin conductance response - SCR). Phasic component is associated with SNS activity in response to stress, tonic component is slower in response to changes associated with external stimulus and is less associated with SNS activity [69]. Subject must present the sum of series of digits before the next light during the mental arithmetic stress test which induces stress and anxiety, resulting in increased SNS and EDA activity [69]. Systolic peak is detected using PPG signal. 2nd order Butterworth LPF at $2 \mathrm{~Hz}$ followed by 2nd order butterworth HPF at $0.1 \mathrm{~Hz}$ was used to filter the PPG signal. HR was obtained through the calculation of intervals between two consecutive systolic peaks. HRV feature was calculated from 5 min baseline and stress features which allowed for the delineation of time and frequency domain features [69]. SD1 and SD2 non-linear variable was obtained from poincare plot which depicts the balance between SNS and PNS [69]. Periodogram with Hanning window was used to compute frequency domain, FFT was applied to each window segment [69]. 4th order butterworth LPF at $1 \mathrm{~Hz}$ was used to obtain EDA time domain features [69]. Signal was then downsampled from 1000 to $1 \mathrm{~Hz}$ in three steps of factor 1/10, then filtered with 8th order HPF at $0.1 \mathrm{~Hz}$ removing trends associated [69]. Periodogram with Blackman window was used to obtain frequency domain features, FFT was applied to each window [69]. LFnu increased and decreased for some subjects during instances of stress [69]. In order to classify stress, SVM used a hyperplane to separate subjects whose $L F n u$ decreased and increased during stress [69]. There was also a contrary decrease in $L F, L F / H F$ ratio during stress 
phases [69]. Using time domain HRV features of HR, RR-interval and $S D 1 / S D 2$ were able classify stress with $80 \%$ accuracy through Random forest classifier [69]. SCL, SCR, rise time from EDA time domain features resulted in 77\% accuracy using RF, stress was obtained by comparing to a baseline for both classifications [69]. These features used to predict stress are not consistent with the theories associated with ANS activity, the classification was obtained by comparing to a baseline signal, and HR which always varies was a prominent predictor of stress in this scenario, classification report which includes $T N, T P, F N, F P$ accuracy behind stress detection would better indicate the reason behind the contradictory results which varies from standard theories behind ANS activity (such as a decrease in $L F, L F / H F$ ratio during times of stress).

2.4h. Stress induced through VR environment and classified using Extreme learning machine (ELM)

Stress is unconsciously regulated by the activity of the ANS, specifically SNS which is stimulated by fight/emergency response from the body. The increase in SNS activity occurs due to various imbalances/defects associated with the function of the body such as increase in blood pressure which can lead to hypertension, increase in HR and low HRV which can lead to cardiovascular pathology and stress which can lead to chronic stress, unless these symptoms are balanced out by the activity of PNS and the function of HRV returns back to normal along with the activity of the ANS. (Abdi et al., 2018) revealed that chronic stress reduces the reaction of ANS activity in response to cognitive load, which is also an important reason to get a good night of sleep otherwise people tend to feel more nervous, worried (more prone to stress), fatigue, they lack coordination, they lack focus, their mind wonders and their more agitated reducing their level of productivity and emotional well-being [59]. (Cho et al., 2017) utilized VR (virtual reality) along with stress inducing task to increase cognitive load (such as mental arithmetic) in order to assess the difference in physiological behaviour due to mild, moderate and severe stress induced (begins from baseline neural activity and includes a recovery period to return their ANS activity back to normal). The experiment required the use of wearable PPG, EDA and ECG sensors to detect changes in blood volume within the peripheral blood vessel, cardiac activity, level of skin conductance (EDA), skin temperature (EDA) and HRV (ECG) respectively. In order to induce mild stress a simple addition/subtraction arithmetic task was performed under a 
VR environment which included soothing beach sound, moderate stress was induced through a dynamic VR environment of a car racing on a rainy day with a similar arithmetic task and severe stress was produced through VR stimuli that can induce fear such as dark, dingy room with

frightening background music along with similar arithmetic task [61]. They were able to classify stress with 95\% accuracy using features obtained from the three physiological signals (PPG, ECG, EDA) through Kernel based Extreme Learning Machine (K-ELM) [61]. K-ELM is based on single hidden layer feedforward neural network which generates input weights and hidden layer biases, it requires less resources to classify results with high accuracy and Leave one out cross validation (LOOCV) was used to evaluate the classifier [61]. The experiment unfolds the possibilities which exist for wireless monitoring of stress, accurate results produced from HRV in motion through a wireless device is an indication of phenomenal solution that is yet to be produced in health care due to the lack of efficiency, this is an indication of stupendous possibilities that may arise within the next decade for wireless monitoring of HRV and human health through the use of machine learning and wearable devices.

\subsection{Applications of HRV Analysis}

HRV has a wide range of applications, some of those applications were presented in Table 1. This section will scrutinize various research experiments which transpired through the analysis of HRV, investigate the changes within a patient's/subject's HRV due to certain activities and morbidities. It will also examine the void and inconsistency of each research study and outline future direction for HRV research, areas which require more attention in order to become a more efficient procedure which can have a positive impact on people's lives and prevent chaotic outcomes.

\subsection{1a HRV and stress}




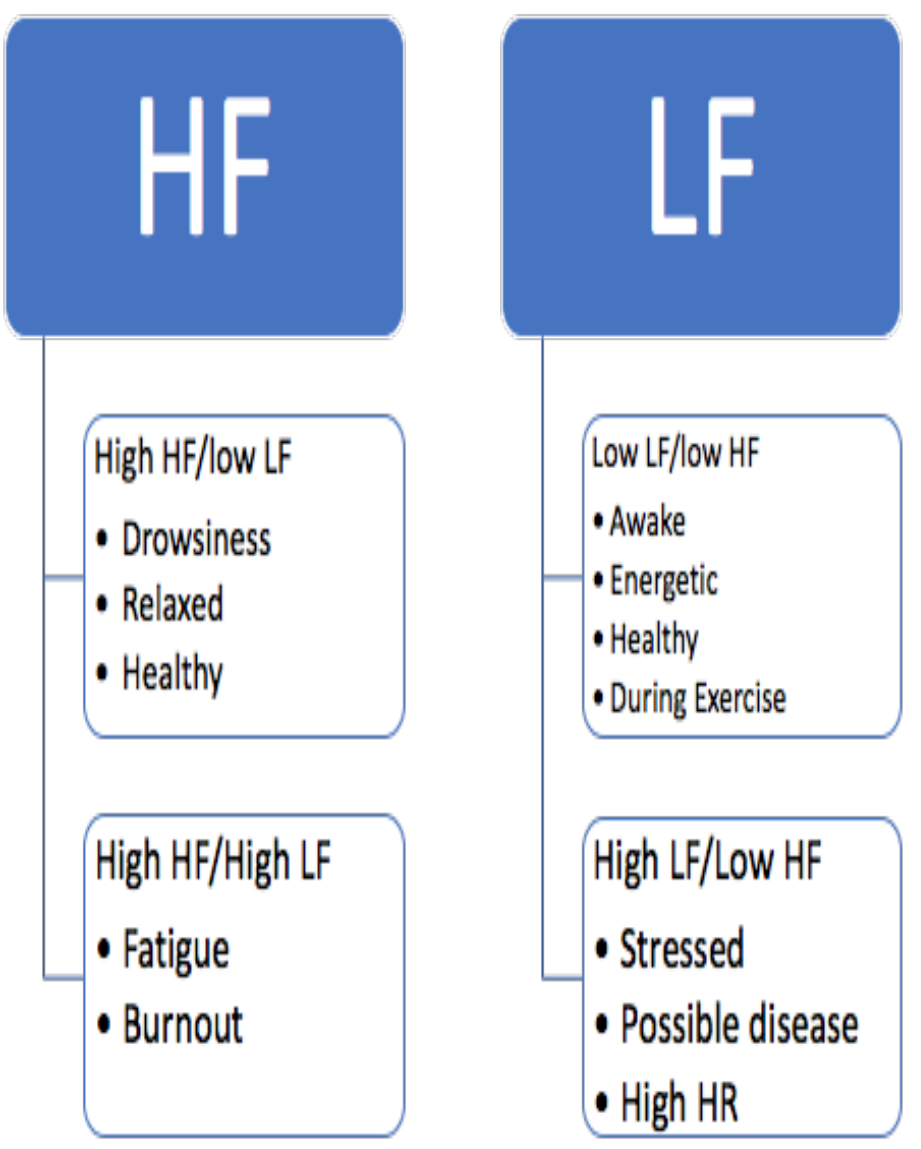

Figure 7: Describes LF and HF associated with stress, drowsiness, awake, fatigue [3].

As described in Figure 7, stress is primarily associated with the activity of the SNS, increased $L F$ (0.04-0.15 Hz) band in frequency domain and reduced HRV. It activates due to perceived danger (such as a deadline, financial worries, exam), increase in cortisol levels causing the activation of SNS which mobilizes the body's activity under stress in order to react/respond rapidly to any dangerous situation [2]. Stress is associated with many situations as indicated by Rosenberg et al [2], whether it is a conference presentation, medical emergency, period of pain, exercise, math, rest, mental stress test or meditation, depending on the activity low-high level of stress is connected to a certain level. High level of stress or physical stress is a cause of morbidity and mortality, negatively affecting the health and daily life of an individual especially patients with myocardial infarction, who suffer the worst consequences due to stress [2][5][6][7]. It impairs a person's decision making ability, sense of accomplishment, increases their HR and blood pressure levels, causes tension within their muscle, overloads biological system (neural, 
mascular, hormonal, cardiovascular), effects their immune system leading to more diseases and infection, reduces neurons in hippocampus, affects memory, cognition, reduces reaction time, and causes anxiety [5][6][7]. There are different levels of stress depending on person's HRV, most often 1D method such as sympathovagal balance ( $L F / H F$ ratio) are used since it is more efficient/simpler than time domain methods (RMSSD, PNN50, SDNN) which would take longer to assess, but the efficiency of the method can be significantly improved [2][3]. Rosenberg et al used a $2 \mathrm{D}$ scatter plot ( $L F$ Vs $H F$ on a $2 \mathrm{D}$ scatter) using multiple variables such as $L F n, L F n$, $L F p, H F p, L F i A, H F i A$ and their ratio and compared it with the $1 \mathrm{D}$ method ( $L F / H F$ ratio or $L F$, $H F$ computed independently) for different stress tests like mental stress, pain, emergency, meditation, and pain and the results concluded that 2D scatter plots were much more efficient than 1D univariate method, 2D results produced accuracy of $90 \%$ or above whereas $1 \mathrm{D}$ method were around 70\% [2]. 1D variable is very linear, unlike stress, it cannot discriminate between 2 test that lead to similar HR like math and exercise, however 2D scatter plot can efficiently differentiate between the ANS activity due to different activities resulting in much more efficient results and categorization of the activity of the ANS (SNS and PNS activity) due to different stress states [2]. The accuracy of the experiment is questionable since only 10 participants were used, which is less indicative of the overall population, one individual can have distinct patterns which is not comparable to the rest of the world during exercise or math, another questionable result would be the result of the HR, exercise should have a higher HR since that's the primary

function of the heart during exercise, an increase in HR for a brief period, resulting in an increased HR upto 5 minutes post exercise. $L F$ value during exercise was also rather low [2], exercise promotes efficient use of one's energy allowing an individual to be more awake/alert throughout the day, which is more associated with the activity within the $L F$ band. A $3 \mathrm{D}$ assessment which includes time would probably result in a more accurate analysis as well [2].

\subsection{1b. Low/Reduced HRV}

Lower/reduced HRV is generally a result of high SNS activity, activity within the $L F$ band, reduced PNS, higher HR/blood pressure which leads to various morbidities and cause of mortality. HRV of Patient/subjects suffering from depression is very low, VLF (0.003 Hz- 0.04 $\mathrm{Hz}$ ) has been positively associated depression and it is also one of the strongest indicators of depression [8]. There are various side effects such as pain, fatigue, digestive problems, distorted 
inflammatory response which are associated with depression or precede depression [8].

Antidepressant does provide short term relief but does not provide a cure or improve HRV, instead it may reduce HRV with prolonged use [8]. ANS is positively correlated to HRV, it allows us to respond effectively to changes associated with the activity of the heart due to certain circumstances or environment. Lower RSA (respiratory sinus arrhythmia) is associated with MDD (major depression disorder), which is an indication of poor emotional regulation, higher levels of stress [8]. Blood et al[8] were able to make these diagnoses using correlation analysis (scatter plots) which compares the activity of the $L F, \mathrm{~V} L F, H F$ due to various symptoms associated with depression [8]. The research study also revealed that low $H F$ (equivalent to low HRV) emanate more anger, sadness, peer problems and anxiety, decreased VLF would cause the development of chronic inflammation, and dysregulation of VLF (associated with metabolic process, thermoregulation, renin angiotensin, regulates blood pressure and fluid balance) would result in more fatigue and depression [8]. The research study did not mention any possible cure for depression, such as exercise or meditation which improves HRV. The research study failed to indicate the importance of nutrition which effects our cognitive function, regulation of ANS and emotional behaviour, as well as HRV in a positive or negative manner depending on the type of food we eat. The study did not specify how an individual's lifestyle, goals, work, reward associated with the amount of effort exerted affected their emotional tendencies and the level of stress associated with depression was not analyzed.

Toni et al exhibited that exercise indeed improves HRV, and if it is affixed with antidepressant, it can significantly affect HRV in a very positive manner [15]. The study was conducted with older aged subject suffering from depression, it also disclosed that antidepressant alone was not an effective method to improve HRV, exercise improves HRV for everyone except fit/healthy population who might notice a slight improvement [15]. Impaired ANS associated with reduced HRV and depression revolves around the dysfunction of cortical and subcortical network located within the prefrontal cortex (decision making) and the amygdala (processes emotion) [15]. Exercise improves vagal tone which improves the function of the prefrontal cortex and decisionmaking abilities [15]. The study did not reveal any graph to indicate how antidepressant and exercise improves HRV, the measurements differed for different subjects who were used to compare the data obtained from exercise and the use of antidepressants, similar attributes would be a more accurate representation for such a comparison. 
Reduced HRV is a risk predictor of heart failure after acute myocardial infarction, a warning sign for diabetic neuropathy, and has been associated with patient suffering from sleep apnea, dilated cardiomyopathy, fetal distress as well as congestive heart failure [13]. Decrease in HRV is correlated to reduced $S D N N$ and a shorter $R-R$ interval [13]. Significantly lower $L F$ along with a reduced HRV can result in sudden cardiac death for patients suffering from $\mathrm{CHF}$ due to the inability of the ANS, which is unable to respond/react accordingly to the treacherous situation [13]. Both time and frequency domain variables (such as $S D N N, L F n, H F n, L F / H F$ ) were used as predictor of morbidity/mortality but the data filtering process, visual representation of the data, effective values associated with the variables were not provided to strengthen the theoretical information presented, leaving doubts and room for improvements within the study conducted by Wang et al.

Moreover, lower HRV and vagal indicated through low $H F$, shorter $R-R$ interval and smaller RMSSD values are associated with epileptic seizure [17]. High SNS activity and lower vagal tone during epileptic seizure, accordingly since SNS activation occurs during threats and danger, high level is required to maintain some level of homeostasis [17]. Changes in ANS during epilepsy results in epileptic death, which is most likely caused by altered SNS activity [17]. HRV was not fully discussed through relevant data, HR during epilepsy was also missing and it would be interesting to know such an important detail concerning epilepsy.

\subsection{1c. $H R V$ and exercise/training}




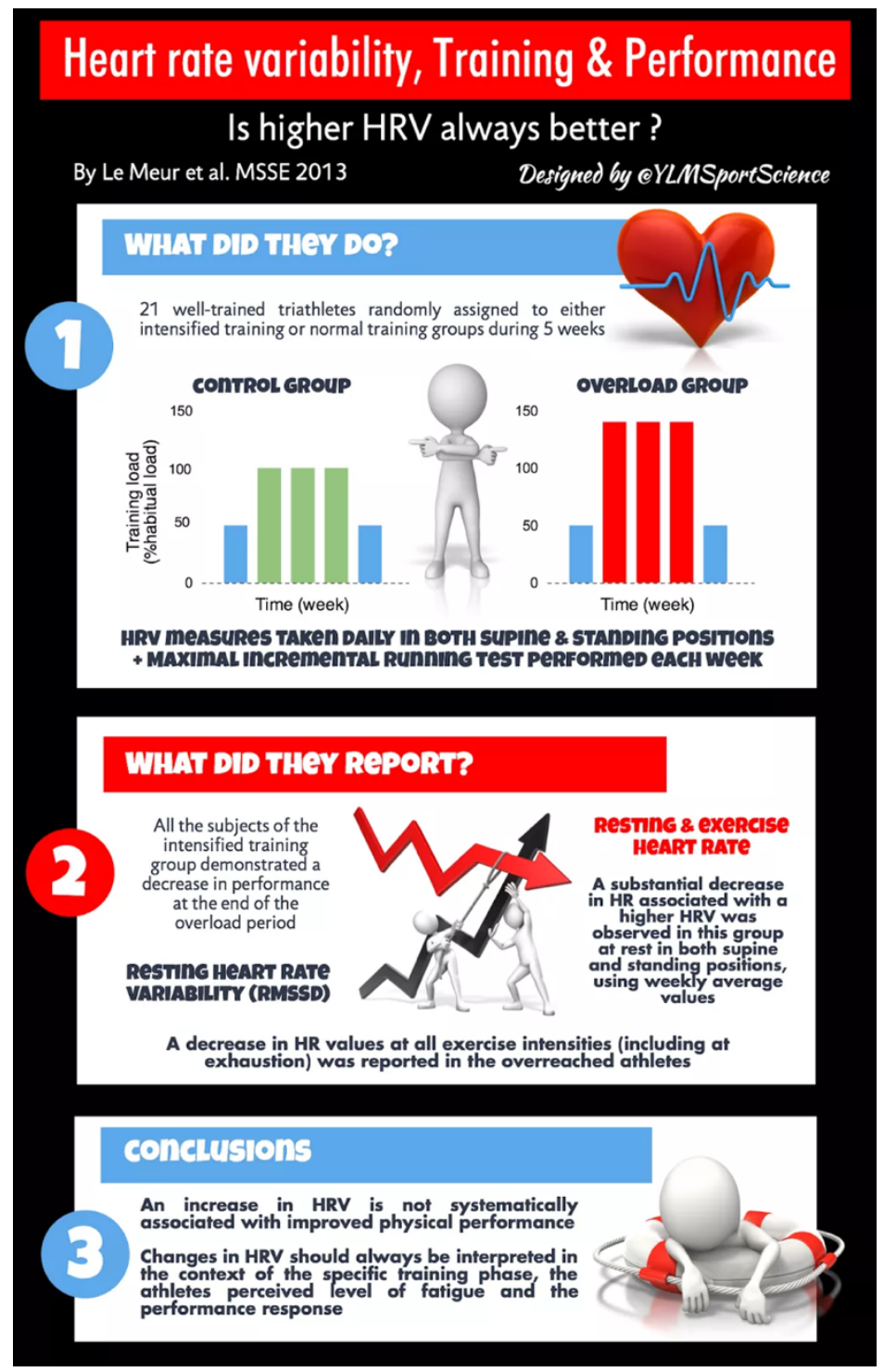

Figure 8: Assessing training performance of athletes through HRV [75]

A study conducted by Shiro et al [9] analyzed the correlation between HRV and chronic neck pain and shoulder pain specifically within female. Increased sympathetic activity ( $L F$ activity) has been associated with stress, but it activates in response to stress in order to maintain homeostasis, indicating that increased stress caused an increase in SNS activity. SNS and ANS help towards maintaining a healthy body, the primary function of the nervous system is to maintain proper function of the body and keep it safe. SNS also contributes towards the maintenance of muscle oxygenation, without it people would suffer from more ache and pain since the body would not be able to break down glucose and use it as fuel for the muscle, lactic acid within the body would build up due to the low amount of oxygen inside the body. ANS 
dysfunction occurs as a result of low oxygen levels and causes neck and shoulder pain [20], lower oxygen and blood flow to the trapezoids. SNS also contributes of vasoconstriction (narrowing of blood vessel) in order to maintain normal arterial blood pressure [9], it is associated with keeping the body safe, increase in pain also increases the activity of the SNS [20]. NDI (neck disability index) increases during pain, due to decreased aerobic capacity of the trapezius and spine as a result of prolonged oxygenation recovery [9]. Common cause of neck and shoulder pain is repetitive/over work which can cause an increase of intramuscular glutamate and lactate within the traps [9]. Isometric contraction was performed to indicate the effect of muscle load, $L F / H F$ was lower (increased HRV) within the relaxed and pain free subject but it was inactive for pain group which was attestation of impaired ANS activity. Inactivity of $L F / H F$ is not a clear and concise representation of ANS dysfunction, 2D scatter plots may have provided more efficient results. The study did not include a clear description of overwork, how many hours of work during each day that would result in lack of oxygenation within the trapezius. Research did not clearly mention how HRV is associated with pain, it reduces due to increased SNS activity but that is only an assumption without verified evidence.

A research study conducted by Leti el al analyzed HRV due to fatigue in order to prevent athlete performance burnout and overtraining [10]. Competition is associated with increased $L F / H F$ and SNS dominance, indicating that athletes may suffer from more fatigue, stress and anxiety during competition [10][18]. The study analyzed senior runners, HRV and $H F$ decrease with an increase in age, aerobic training positively impacts $\mathrm{HRV}$ and $H F$ indicated through the positive correlation with time domain parameters such as $S D N N$ and $R M S S D$ and $H F$ [10]. Excessive training can cause impairment of the cardiovascular control system, negatively impact a competitor's mood/state which has been associated with injury and fatigue, a result of reduced HRV [10]. Increase in SNS compensates for reduced cardiac performance and helps recover normal blood flow [10]. High SNS is also associated with fatigue during training which correlates to reduced HRV [10][18]. Two days after the competition, PNS activity and HRV increases, indicating that training improves vagal tone and helps to maintain ANS modulation. The research study only studied 10 athletes which is less indicative of the overall population. The filtering process of the data was not succinct, also a brief summary of their training background which could indicate their prior level of health, experienced athletes are very different from the 
average population, their cardiac activity produced results which are different in comparison to an inexperienced athlete. Including control subjects (normal people to compare with) would produce more efficient results that can provide an important feedback to the general population/fitness enthusiasts.

2.5.1d. HRV and Cardiovascular system

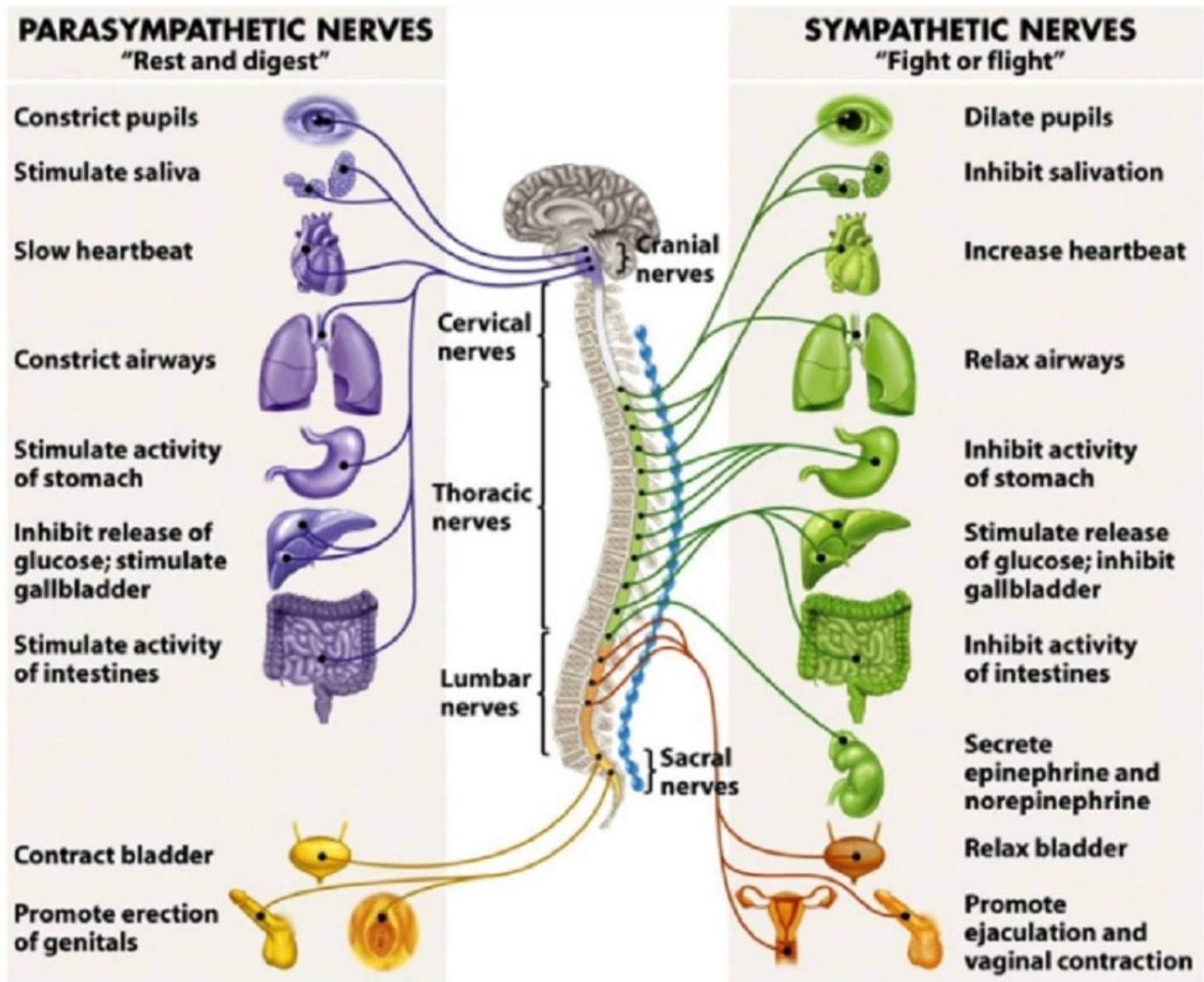

Figure 9: PNS and SNS activity associated with HRV[71]

HRV represents how efficiently the cardiovascular system is functioning, increased HRV can keep up better with changes associated with the activity of the ANS, a healthy ANS is responsible for modulating exercise capacity in patients with cardiac syndrome $\mathrm{x}$ [11]. PNS withdrawal and reduced HRV is associated with reduced coronary flow reserve which reduces resting blood flow causing the myocardium to be more perceptible to ischemia (blood clot) [11]. 
Lower HRV/PNS is also associated with reduced exercise capacity, higher BP, higher pulse pressure, decreased muscular blood flow and oxygen supply which is also responsible for chronic pain [11]. The data analysis was very theoretical, there were no visual graph or frequency domain analysis to provide an accurate perusal.

Environmental noise such as rail, aircraft, road traffic is associated with reduced HRV and stress due to decrease of hormone cortisol leading to more bad decisions as well [12]. Exposure to noise causes an increased blood pressure in response which results in cardiac comorbidities such as hypertension, ischemic heart disease and myocardial infarction [12]. Decreased HRV is less capable of reacting to the changes associated with increased stress rendering people's ability to move as smoothly or react/respond accordingly to certain situations [12]. The data did not present a visual graph which would be a clearer delineation of the activity of HRV in frequency domain, the data was also minimal with 10 subjects, making it more skeptical and based on assumption obtained from general theory associated with HRV.

Pinheiro et al disclosed a study which analysed cardiac dysfunction within older aged women due to reduced HRV [14]. Higher probability/occurrence of myocardial infarction associated with older women as a result of low HRV and ANS dysfunction [14]. Data also revealed that $S D N N, R M S S D$, triangular index were significantly worse for women than men, additionally reduced HRV is the strongest predictor of myocardial infarction [14]. Resting HR is a robust indicator of myocardial infarction and coronary death within women, low HR as well increased HR associated with depression antedates coronary artery disease [14]. Women and men require different treatments for an accurate prognosis due to sexual dimorphism associated with men and women [14]. More relevant data along with the filtering process as well as blood pressure /HRV measurements would effectively verify the information presented.

Patients suffering from stroke and requiring hemodialysis also indicated a lower HRV [23], post dialysis presented an increased $\mathrm{V} L F, L F, T P$ and $L F / H F$ ratio which stipulate that the treatment allows them to be safer, alter VLF and $L F$ might result in cardiovascular death [23]. VLF is robust in terms of prognosis for CHF. Lower HRV is also associated with adverse cardiac states, increased morbidity and mortality within patients suffering from ESRD (end stage renal disease) 
[23]. What is the possible cause of stroke, what is their emotional state prior to stroke, comorbidities associated with stroke have yet to be answered?

Amaral et al identified that music can increase or reduce HRV based on the type of music and its impact on HRV [29]. Music therapy involving soothing music which improves HR has been utilized to improve cardiac function after taking cardiotoxic medication [29]. Heavy metal and metal rock reduced HRV and the modulation of the heart indicated through reduced SDNN [29]. Relaxing music such as classical music improved HRV in patients with cardiovascular dysfunction, dementia, [29]. Interestingly classical music at high intensity also reduced HRV, although sufficient analysis was not provided [29]. $L F$ was reduced during heavy metal which may indicate that it is harmful and causes increased fatigue [29]. Higher intensity of music increased sympathetic tone on HR, the reaction designates that music is perceived as a threat by the ANS and may induce stress/fatigue [29]. Relaxing music improved dopamine levels, blood flow to the brain and arterial blood pressure [29]. There are still questions which remain unanswered or is not properly specified such as: is baroque really harmful, it is a classical type of music and what are some of the emotional side effects of heavy metal (such as stress, fatigue, drowsiness,).

2.5.1e. HRV and Drowsiness 

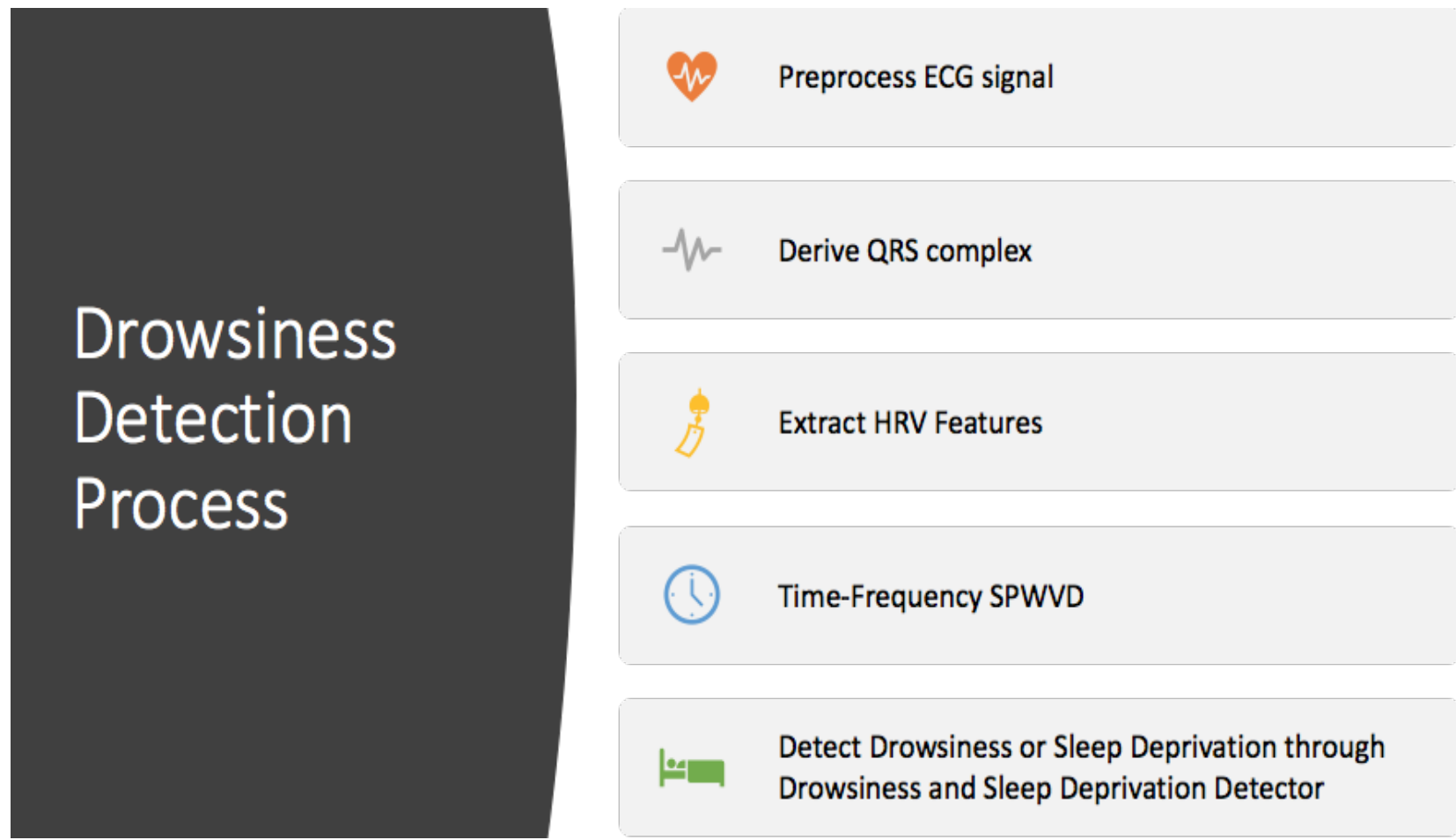

Figure 10: Process behind drowsiness detection through HRV [3].

Unlike other applications stated above which are morbid conditions associated with reduced HRV, drowsiness is related to increased HRV and reduced $L F / H F$ ratio. Transition to sleep from an awake state results in an increase in HRV, where the PNS activity is superior in comparison to SNS in order to modulate the body's activity during sleep/rest. Around 10-30\% of all road crashes are associated with fatigue and drowsy driving and recent smart watches and portable ECG are efficiently being utilized to antedate drowsiness in order to alert the driver prior to any possible accidents. Accelerometer and gyrometer are tested to assess the users HRV and physical activity which allows for the detection of drowsiness/fatigue prior to the transition to stage 1 sleep (drowsiness) [30]. Fatigue and drowsiness have been associated with the time of the day, nighttime are especially bad for driving, drowsiness is associated with increased HRV which is also related to reduced SNS and reaction time, dictating that drowsy driving is horrendous during the night time [30]. There is a high correlation between PPG and ECG in terms of detecting HR, Lee et al proposed a method to automatically remove noise from PPG using a PPG strap which can be used to detect accurate HR while driving, PSD can be utilized to detect HRV in frequency domain, making it a simple and effective method to detect drowsiness through a person's HR [30]. Although the HR alone is not sufficient to detect drowsiness which involves multiple other 
components like the last period of sleep, accurate HRV measurement, amount of sleep prior to driving. Mohammed et al stated that $R-R$ interval increases $22 \%$ in comparison to a normal driver during a drowsy period [32]. Physiological signals such as an ECG has been described as the most accurate representation of drowsiness in comparison to vehicle-based method (lane position of the vehicle) and behavioural method (yawning, eye blinking) [33]. Although PNS is more active during sleep period, during REM sleep SNS activity increases beyond the activity of the PNS [33]. $L F$ reflects blood pressure control, $H F$ represents respiratory rhythm and positively correlates to $\mathrm{HRV}, H F$ gradually increases from awake to sleep, $L F$ gradually decrease during NREM sleep, SNS is dominant during awake and REM period [33]. Purnamasari et al published that $L F$ also increased from $679 \mathrm{~ms} 2$ to $1328 \mathrm{~ms} 2$, significant increase in $H F$ during drowsiness from $163 \mathrm{~ms} 2$ to $980 \mathrm{~ms} 2$ and a drastic decrease in $L F / H F$ during drowsiness from 4.164 - 1.355, which was obtained through virtual reality simulation [45].

New revelation (pilot study, not developed) such as using an ECG sensor to detect drowsiness while the driver is driving, GSM module can be utilized to send continuous signal to control room, DC can be used to control the speed of the vehicle upon drowsy detection since the drivers reaction is distorted, LCD is used to monitor drivers condition and LED in the rear side of the vehicle used to signal the vehicle behind the drowsy vehicle to slow down [34]. Roy et al proposed a similar idea using an accelerometer to detect motion, SMS to send an alert message to the control room, microcontroller is used to process the analog signal which can be analyzed through LabVIEW, Matlab[35]. Drowsiness is still relatively new in comparison to myocardial infarction and hypertension which has been studied for over 30 years, which is one of the biggest reasons for lack of adequate research concerning drowsy driving, an accurate method to detect drowsiness is still a part of ongoing research, it makes sense in theory but HRV is complex and becomes more intricate to detect in motion such as exercise (only $78.6 \%-85 \%$ accuracy in frequency domain) [37] and especially worse during drowsy driving. Vicente el al conducted a study which involved truck drivers using a drowsy detection detector as well as a sleep deprivation detector, and the accuracy of the results were 0.59 and 0.62 sensitivity, respectively [3]. The results were in frequency domain, it indicates that when a truck is in motion there are a lot of error associated with wireless ECG detection, some parts of the signal were blank indication of FP associated (failed to detect) with signal detection while in motion. Specificity and predictivity were 0.98 and 0.96 using a drowsiness episodes detector, 0.88 and 0.80 using a 
sleep deprivation detector, disclosing that detection of the signal was the hardest part during this process, specifying drowsiness/awake state upon detection was very accurate through the data analysis of ECG using linear discriminant analysis (LDA) classification method.

2.5.1f. Software to analyse $H R V$

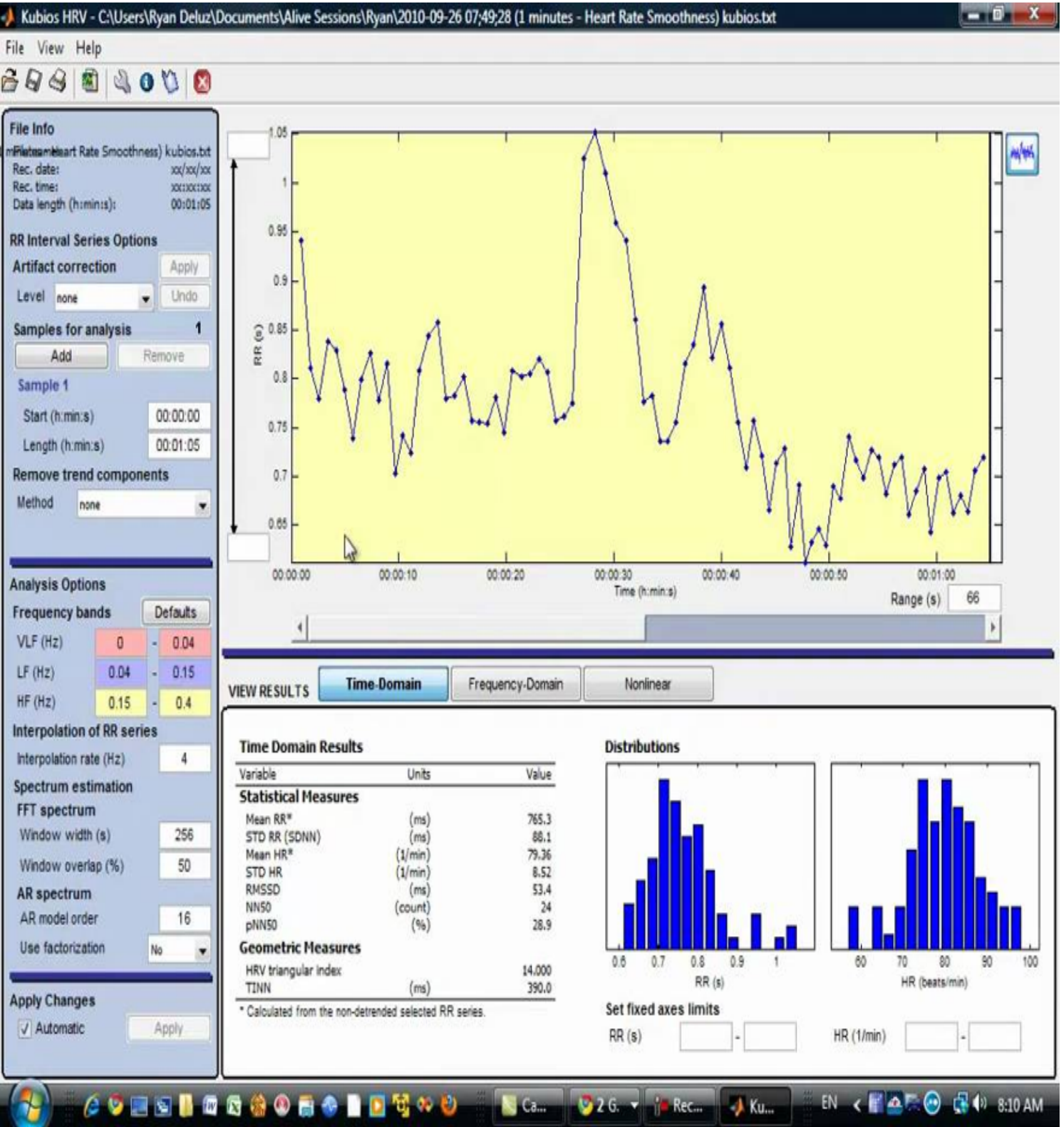

Figure 11: HRV analysis using Kubios Software 
There are various tools to detect an ECG signal such as wireless watches (Fitbit, apple watch), wireless ECG sensor with Ag/Cl electrode, analog circuit, ECG monitor (typically in hospital), straps (zephyr). The detected signal can be digitally analysed through various applications on a computer such as MATLAB, LabVIEW and now recently androids are incorporating apps which can analyze a person's ECG signal obtained from a smartwatch or wrist strap. Those methods are time consuming, rigorous and expensive, HRV has become a popular research study used to analyse an individual's ANS activity, a research group from Finland created Kubios software which can automatically measure and filter ECG signals to delineate HRV, Marcus Vollmer created a similar tool to assess HRV called HRVtool, John Ramshur from Memphis developed HRVAS (HRV analysis software) which can automatically analyze HRV from an ECG signal. The drawback of the HRV toolbox are its automatic filters, which are unreliable and may induce/include more errors [37].

2.5.1g. Bridging the gap between Human physiology and video game.

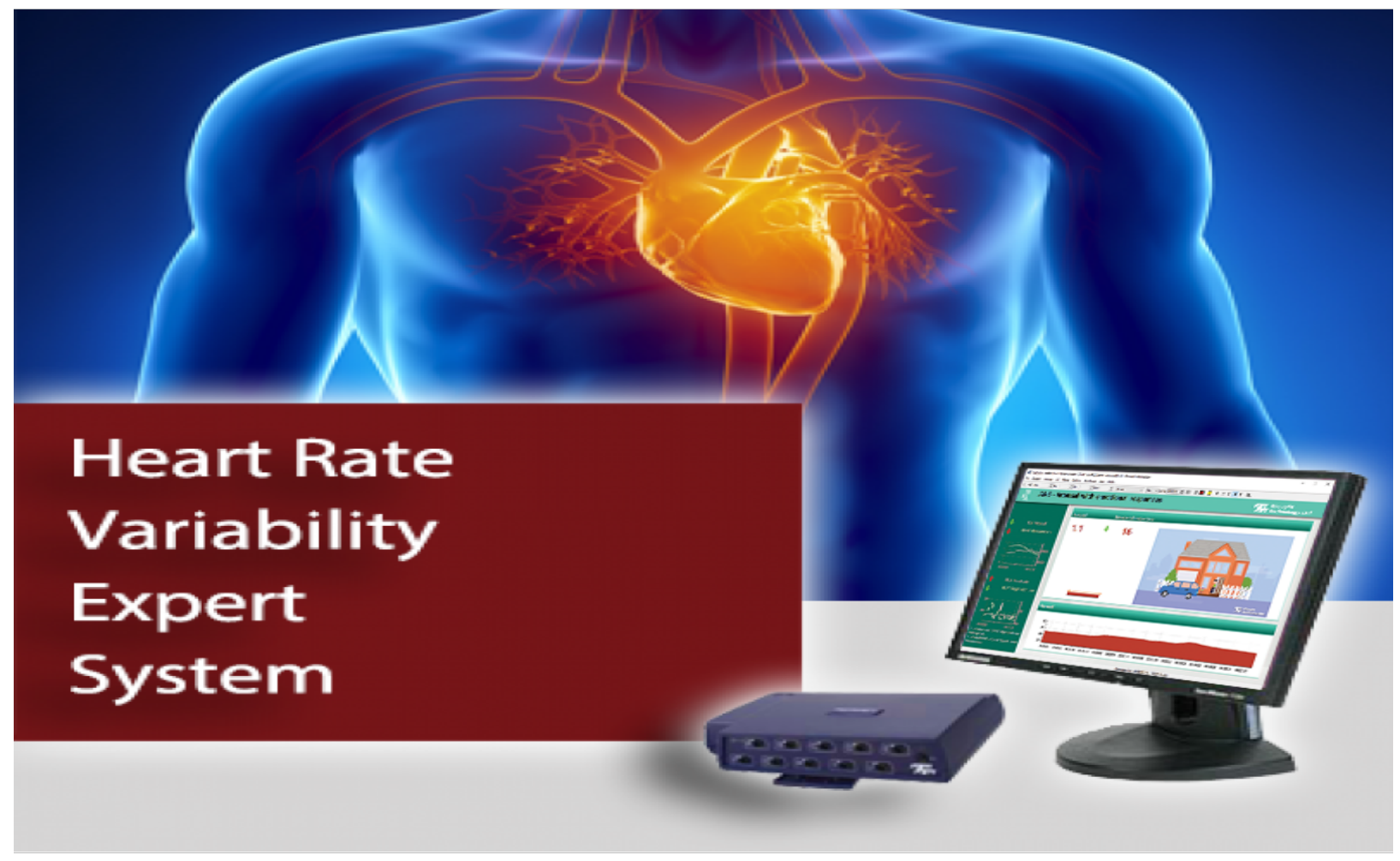

Figure 12: A system to detect and analyze HRV [76] 
HCI (human to computer interaction) is one of the various methods utilized for stress analysis, cognitive games such as stroop test are tailored to induce stress, indicating that one of the biggest causes of stress is associated with the inability to complete a task, whilst most of the worrying occurs prior to a big task, due to fatigue, fear of criticism (leaves the impression that the task was not efficiently done) and lack of good night sleep. (Adam et al., 2015) developed a novel method in order to design a video game FlappyHeartPC using ECG signal as the input, bridging the gap between human physiology and gaming, such interaction might spark more interest within the user for a boring activity (which is relaxing and beneficial for stress reduction health) such as mediation, fishing or simply analyzing your physiological signal in a lab [68]. The game design includes a tailor belt worn below the chest with electro-textile electrodes was used as the interface between the sensor and the skin, data acquisition required Bitalino (a specialized data acquisition board), python was used to design the signal processing algorithm used to process/filter the input ECG signal, detect QRS complex and calculate HR [68]. Unity 3D was the engine which made the development of the game possible which can utilize HR as the input for certain physiological analysis [68]. $50 \mathrm{~Hz}$ FIR Notch filter along with $20 \mathrm{~Hz}$ LPF (order 61, Blackman window) and $15 \mathrm{~Hz}$ HPF (order 61, Blackman window) were applied to filter DC component and powerline interference, differentiator and wavelet peak detector were used for peak detection through scipy.signal python library [68]. Microsoft Kinect combined with electrodes were used to represent the body in virtual reality. QRS was detected from electrocardiogram signals. Wavelet algorithm obtained $91.4 \%$ accuracy with $95.9 \%$ precision while differentiation algorithm obtained $90.6 \%$ accuracy and precision [68]. The video game is a great innovation which can be utilized for science and excitement but it did not have a specific purpose outside of the gaming business, it can be utilized to improve human health by implementing stress detection algorithm into the game, if ML learning can be embedded there are various possibilities with regards to health such as predicting stress, CVD, hypertension .

\subsection{1h. Future direction for $H R V$}

HRV is a prominent topic concerning the activity of the heart and the ANS, but although research has been steadily increasing, data analysis of HRV in motion is far from where it should be especially concerning drowsiness. Vincente et. al. [3] and Giorgiou et. al. [37] have explained 
that HRV is hard to detect in motion whether it involves exercise or drowsy driving, accuracy of HRV measures declines due to motion. Detection method in motion is a concern and should be a priority for improvement with regards to future research involving HRV. Machine learning algorithm, Frequency domain analysis are better but researchers should continue to expand their knowledge towards these aspects of data analysis which would provide effective/efficient results that produce an accurate representation of a person's HRV, easy to compute and can analyse a lot of data at once making the detection process a lot smoother and quicker.

\subsection{Stress and Video Games}

Stress is prevalent all over the world, chronic stress is a dreadful development which occurs due to prolonged stress. Chronic stress can lead to many lethal cardiac diseases such as myocardial infarction, hypertension, diabetes, obesity and accelerated aging/premature death. Researchers are analyzing effective methods to increase stress resilience and manage stress. Video games recently has gained recognition for its ability to improve social skills, stimulate mental activity and increase stress resilience. Aliary et al. [98] studied the beneficial and harmful effects of video games on cognitive functions. They analyzed the function of alpha-amylase, cortisol and brain waves due to stress using EEG signals. The results indicate that depending on the type of video games, stress level varies as well. Non-violent games which can stimulate mental activity such as tetris are beneficial for stress levels. Roy et al. [99] studied the impact of competitive and cooperative video games on stress levels. The results obtained from HR, BP, se $L F$-reported questionnaire on stress and valence indicate that video games can improve social skills and reduce stress. The group conveyed the message that video games are a great way to socialize with one another, play cooperatively and distract people from stress. Socializing is also a great to reduce stress levels. Reinecke, el at. [100] conducted an experiment to analyze whether strain is an effective way to recuperate from work stress. Upon analyzing frequency of game use, recovery time, relaxation and daily hassle they came to the conclusion that video games can in fact help people recover after being exposed to stressful situations. Although realized that if video games are used appropriately within limits, it is a great way to help working individual recover from stress. Research using video games are steadily increasing but machine learning and physiological signal analysis within this domain is almost non-existent. This thesis covers that gap, discovers the impact of video games through physiological functions and classifies 
stress using machine learning. Research studies should incorporate signal analysis to study the effect of video games on stress levels, it is one of the most addictive activities which may indeed prove to be beneficial, if it is utilized the right way.

\section{Chapter 3}

\section{Physiological Signal Analysis}

\subsection{Introduction}

Stress is one of the most common causes of several health problems occurring throughout the world. It is associated with many human activities such as our jobs, our social relationships, sports and our finance. Stress can be considered as a positive symptom associated with human physiology, it helps us focus, makes us feel exhilarating when we push ourselves to meet a deadline, it makes feel energetic, alive and ready for an upcoming challenge due to our blood vessels dilating which increases blood flow to the brain, muscles and limbs [82]. The aforementioned symptoms of stress are associated with adaptive/eutstress which is considered to be good since it allows people to perform at their peak level [82]. Human body reacts in response to stress, a person's mental, physical and emotional responses are adjusted to continuously resist stress until the body adapts to the stress placed upon it [82]. Prolonged stress transitions the body into its second resistance state (past acute stress but still considered beneficial) which requires continuous effort to resist and remain in a state of arousal (eustress which is good and makes a person feel energized during work) but if the resist to stress is exhausted (bodies exhaustion stage), it leads to a person suffering from distress [83]. Distress is the harmful type of stress, it's a lot different from eustress (state of arousal, energetic), the blood vessels constrict during this phase, resulting in a person transitioning to a state of rage/anger, voice becomes louder, and it impairs a person's cognitive function, physical health thus reducing the level of productivity and decision making ability [82]. Distress leads to heart beating irregularly (above $100 \mathrm{bpm}$ or it may be too low below $60 \mathrm{bpm}$ due to fear), prolonged stress can suppress the function of the immune system, inhibit inflammatory response, increase blood pressure, damage muscle tissue, which severely damages the function of the body [83]. Early detection of stress along with established methods to better manage stress can positively impact the health of millions of 
people around the world and reduce the risk of major cardiovascular diseases such as hypertension, diabetes, myocardial infarction. The purpose of this research experiment was to assess stress by inducing it within subjects through a roller coaster and color stroop test cognitive game. The experiment was broken down into 4 phases: 1) baseline phase (recording of their signal while they are normal) 2) roller coaster phase (the purpose was to induce stress) 3) colour stroop test (increase stress through a cognitive game) 4) VR fish game (purpose was to relax the subject after inducing stress). Their physiological signals (ECG, GSR, RESP) were acquired through captiv wireless sensors such as ECG sensor, GSR sensor and RESP sensors. ECG sensor collected information about the electrical activity of their heart which was used to obtain time and frequency domain parameters associated with HRV. GSR sensor was used to analyze their EDA activity (electrodermal activity) and RESP sensor was used to collect their respiration rate. The results can indicate how stress is induced due to the challenges associated with everyday life due to work, financial problems and emotional trauma. The experiment explores whether virtual reality video games are a valid solution to manage stress and prevent distress which can be utilized to develop apps, games and algorithms that can improve the health of others through entertainment.

\subsubsection{Physiological Signal Analysis}

The time domain features utilized to analyze HRV as follows:

\section{RMSSD}

The root mean square of successive differences (RMSSD) between normal heartbeats is equivalent to nonlinear performance metric $S D 1$, both are utilized to analyze short-term HRV [81]. Generally, 5 minutes recording windows are used to analyze HRV but RMSSD can be analyzed through ultra-short-term periods which ranges from 0-2 (minutes) such as $10 \mathrm{~s}, 30 \mathrm{~s}, 60 \mathrm{~s}$ or 2 minutes [81]. RMSSD is primarily manipulated by PNS (parasympathetic nervous system), it shares a correlation with $H F(0.15-0.4 \mathrm{~Hz})$, it is strongly correlated to PNN50 (percentage of NN interval greater than $50 \mathrm{~ms}$ ) and it used to assess changes within the vagus nerve due to HRV [81].

$$
R M S S D=\sqrt{\frac{1}{N-1} \sum_{j=1}^{N}\left(R R_{j+1}-\overline{R R_{j}}\right)^{2}}
$$


It is calculated by obtaining the successive time difference between heartbeats in ms ( $R R$ interval difference), which is squared, and the corresponding result is averaged prior to the square root of the total value obtained [81]. Although RMSSD shares a strong correlation with $H F$, respiration rate cannot be predicted from it, since respiration rate has minimal impact on RMSSD value [81]. $R M S S D$ is primarily used to indicate $H F$ values and HR oscillations. Increases in RMSSD is generally correlated with a decrease in stress levels [81].

\section{$S D N N$}

$$
S D N N=\sqrt{\frac{1}{N-1} \sum_{j=1}^{N}\left(R R_{j}-\overline{R R}\right)^{2}}
$$

The standard deviation of NN interval $(S D N N)$ measures the total power with respect to the segment being analyzed, it is used to represent normal sinus rhythm and it is measured in terms of ms [81]. The statistical representation of $S D N N$ can be obtained through the root mean-square of successive differences of adjacent intervals (RMSSD) [81]. Although in most general cases short-term recording periods are 5 minutes in duration, $S D N N$ can be analyzed efficiently through 1-3-minute ultrashort-term windows [81]. $S D N N$ shares a high correlation with $L F, \mathrm{~V} L F$ and $\mathrm{U} L F$ band power and total power, depending on the experiment and biological condition, the correlation may vary [81]. If the power within those bands are greater than $H F$ bands, they share a stronger correlation with $S D N N$ [81]. Slow paced breathing primarily affects the vagus nerve and it is stimulated by the parasympathetic nervous system; it causes variations in $S D N N$ during short-term recordings [81]. $S D N N$ is very efficient during long-term duration such as 24 hours duration, $L F$ shares the strongest correlation with $S D N N$ for 24 hours duration [81]. Biofeedback systems prefer long-term durations in order to produce more efficient $S D N N$ results and cardiac reactions, since environmental stimulus from a higher range often requires a longer period [81]. $S D N N$ can be used to indicate cardiorespiratory regulation, long periods are required to describe the relationship through dynamic workloads and the associated reaction of the heart [81]. It can also be utilized to understand circadian rhythm which dictates a person's sleep/wake cycle, during 24-hour periods, it can efficiently indicate the volume of SNS activity associated with HRV [81]. One of its most important attributes involves discriminating cardiac risks during medical emergency over 24 hours recording period, it can be utilized to classify and predict morbidity/mortality [81]. Subjects whose results display $S D N N$ values less than $50 \mathrm{~ms}$ over a 24 
hours period are classified as abnormal cardiac health, 50-100 $\mathrm{ms}$ is an indication of a patient being at risk of developing cardiac morbidity, and if the $S D N N$ value is greater than $100 \mathrm{~ms}$, they are classified as normal patients [81]. An increase in stress levels generally results in a decrease in $S D N N$ values, immediate changes are often associated with an increase in stress.

NN50

Represents the number of $R-R(N-N)$ interval which varies by $50 \mathrm{~ms}$ between each other and efficient results often requires an epoch of at least $2 \min [81]$.

pNN50

Represents the percentage of the difference associated with $N N$ interval which differ more than $50 \mathrm{~ms}$. A 2-min epoch should be utilized, although ultra-short periods of 60 seconds has been used by researchers [81]. It shares a strong correlation with PNS activity, RMSSD, HF respectively [81]. During stressful situations, increased levels of stress often leads to a decrease in $p N N 50$ and reduced variation within HRV [81].

The Frequency domain parameters used to analyse HRV are listed below:

\section{VLF}

Represented within the VLF band (0.0033-0.04 Hz), 5 min recording period should be utilized for efficient results associated with $\mathrm{V} L F$, higher recording lengths result in more accurate values [81]. It is correlated to mortality within patients, normal rhythm indicates normal health [81]. Low values of VLF often indicate arrhythmic death (44), associated with abnormally high heart rate, high inflammation $(53,54)$ [81]. Low VLF is also correlated with low levels of testosterone. The amplitude and oscillation frequency associated with VLF is mediated by SNS activity. Heart rhythm impacted by physical activity, thermoregulatory affects the VLF power [81]. SNS activity within subjects suffering from tetraplegia are impaired and cannot innervate the heart and lungs [81]. Different sensory neurons within the heart shares an apparent correlation with VLF rhythm, its amplitude and frequency are stimulated by an increase in stress [81].

\section{LF}


$L F$ is represented through $0.04-0.15 \mathrm{~Hz}$ within the PSD, a minimum of 2 minutes is required to achieve precise values associated with an autonomic activity [81]. LF band is often used to indicate baroreceptor (pressure changes) activity while a person is at rest [81]. It is associated with the activity of both the PNS and SNS [81]. It is mostly used to specify the activity corresponding to the sympathetic nervous system, parasympathetic nervous system can stimulate heart rhythms which occurs within the $L F$ range due to slow breathing (below $8.5 \mathrm{bpm}$ or $7 \mathrm{~s}$ periods), deep breaths or sighs [81]. Stressful situations often increase HR and SNS activity, which is associated with an increase in $L F$ values.

\section{$\boldsymbol{H F}$}

$H F$ is represented by the frequency range of $0.15-0.40 \mathrm{~Hz}(0.24-1.04$ for children and adults who breathe faster), it requires a minimum of a 1-min recording duration [81]. $H F$ solely indicates PNS activity and often specifies respiration rhythm which corresponds to variations in HR [81]. HR increases during inhalation and decreases during exhalation due to the release of acetylcholine [81]. $H F$ power shares a strong correlation to RMSSD and $p N N 50$. Low $H F$ values (impaired PNS) is associated with cardiovascular pathology (myocardial infarction, hypertension) and morbidity [81]. HF often decreases (has a low value) due to stress, anxiety, and worry[81].

\subsubsection{Non-Linear Analysis, GSR, Respiration \\ Poincare plot}

Poincare plot are ideally used to analyze the impact of cardiac morbidities associated with impaired HRV. It shares an $81 \%$ correlation with $L F / H F$ ratio [101], which has been proven to be the most effective method to analyze HRV/stress. This is the first time it is utilized to analyze stress from a VR (virtual reality) simulation and VR video game. Poincare plot represents a scatter plot of successive $R-R$ intervals, each as a function of the previous $R-R$ interval. It's a visual analysis which represents the variation within $R-R$ intervals [81]. A poincare plot process flow chart is displayed below in Figure 14.

\section{$S D 1$ and $S D 2$}

$S D 1$ and SD2 are non-linear variables derived from the Poincare plot, they are used to derive total HRV and share a correlation with $L F, H F$ and RMSSD. RMSSD and SDI are used to derive 
short term HRV [81]. Longer epoch of about 5 min can be utilized to predict maximum HR, minimum HR, total power, diastolic blood pressure through SD1 [81]. SD2 shares a correlation with $L F$, it is used to represent both short-term (2-3 min duration) and long-term (24 hours) HRV [81]. In order to assess autonomic balance, the recording period should be long [81].

\section{GSR}

GSR is used to monitor Electrodermal activity. It indicates the changes associated with electrical properties of the skin due to sweat secretion [81].

\section{Respiration}

Respiration also known as the act of breathing, may increase PNS activity/ $H F$ and decrease $L F /$ SNS activity.

\subsubsection{Power Spectral Density}

\section{AR model - Parametric Method}

Autoregressive model is a time series model which computes the spectral estimates of the HRV, it accomplished the desired depiction of PSD and prediction using few variables [84]. AR model is capable of achieving efficient frequency resolution and computing statistical probability of HRV data using short epochs. The order of the AR model is a prominent factor, it needs to be clear and detailed, the order impacts and may compromise the frequency resolution along with statistical stability. It provides a more efficient PSD estimation with a more efficient spectral resolution and less spectral leakage in comparison to non-parametric and classical spectral estimation techniques [84].

$$
X(n)=-\sum_{i=1}^{p} a(i) x(n-i)+w(n)
$$

AR method is parametric, it includes an input of white noise which is filtered through an infinite impulse response filter [56]. The equation of the AR method along with the order $\mathrm{p}$ is listed above. In the equation $a(i)$ represents autoregression coefficients and $w(n)$ represents White Gaussian noise. Order 14 was used to compute PSD of HRV for this research study. This was the most optimal order which led to the best temporal resolution and depiction of the AR PSD. Various orders were applied prior to the selection, increasing the order reduced the prediction error and 14 led to the least $A I C$. AIC is computed through subtracting twice the logarithmic of 
the likelihood function from twice the order. $A I C$ is a method to reduce error associated with a signal wave by regulating cancellation subcarriers associated with the signal [102]. Although higher order leads to less prediction error, the order must also be less than or equal to half the input frame size and the accuracy decreases after increasing the order beyond 14.

The yulear method was utilized to acquire the AR model parameter, it returns the power spectral density estimate of a discrete-time signal. The Power spectrum of a $k$-th order AR model is mentioned below, where $a_{1 \ldots} a_{m}$ represents the model coefficients and $r^{2}{ }_{v}$ represents the input white noise.

$$
r(l)=-\sum_{k=1}^{M} a_{k}^{*} r(l-k)=\sum_{k=1}^{M} w_{k}^{*} r(l-k) \quad\left(w_{k}=-a_{k}\right)
$$

AR model can perform spectral performance very smoothly with ease, it can be used to analyze complex HRV data, due to its ability to handle heart period sequences which includes non-sinus and other unusual beats (ectopic beats) [85]. It can perform spectral analysis of unevenly sampled data and missing data without producing distorted results which makes the PSD computation more efficient in comparison to other established PSD methods [85].

\section{Lomb Scargle Periodogram}

Contrary to other established methods that is used to analyze the PSD of periodic signals, Lomb Scargle Periodogram can be utilized to assess uneven-sampled data. It can perform signal analysis on signals which include non-uniform intervals, it works smoothly with $R R$ time series data, which meshes perfectly with the corresponding signal analyzed via lomb-scargle method [87]. The model estimates the signal's energy by fitting (least squares) sinusoidal model to the signal, which centers the signal through frequency, f with one frequency-band [87]. The equation listed below represents the model used to fit the non-uniform sequence of a signal $\{t n, x(n)\}$ [87].

$$
P_{n}\left(a, b, f, t_{n}\right)=a \cdot \cos \left(2 \pi f t_{n}\right)+b \cdot \sin \left(2 \pi f t_{n}\right)
$$

The estimation error of the signal is depicted through the equation: 


$$
E=\sum_{n=0}^{N-1}\left[P_{n}-x(n)\right]^{2} \quad \frac{\partial E}{\partial a}=0, \quad \frac{\partial E}{\partial b}=0
$$

The periodogram surrounding $\mathrm{f}$ is calculated through:

$$
\begin{gathered}
S(f)=\frac{\left[\sum x(n) \cdot \cos \left(2 \pi f\left(t_{n}-\tau\right)\right)\right]^{2}}{\sum \cos ^{2}\left(2 \pi f\left(t_{n}-\tau\right)\right)}+\frac{\left[\sum x(n) \cdot \sin \left(2 \pi f\left(t_{n}-\tau\right)\right)\right]^{2}}{\sum \sin ^{2}\left(2 \pi f\left(t_{n}-\tau\right)\right)} \\
\tau=\frac{1}{4 \pi f} \tan ^{-1}\left[\frac{\sum \sin \left(4 \pi f t_{n}\right)}{\sum \cos \left(4 \pi f t_{n}\right)}\right]
\end{gathered}
$$

Where $\tau$ represents a delay, which allows the sinusoids to be mutually orthogonal [87]. 1-2 minutes windows $T$ are used to analyze neonatal ECG [87]. Inverse $(d f=1 / T)$ of window $T$ represents the lowest independent frequency $(d f)$ that can be analyzed [87]. Generally, the frequency resolution of the signal is $1 /(4 T)$ which requires the spectrum to be oversampled by a factor of 4 , which is equivalent to $4.2 \mathrm{mHz}$ if the window length is 1 minutes [87]. Lomb algorithm is able to overcome the limitations of FFT with regards to PSD computation such as artifacts (error associated with the signal due to electrode, environment, movement), stationarity ( fixed position), evenly sampled data, sampling frame issues which distorts near-real-time analysis [87].

\subsubsection{Preprocessing/Filtering}

Digital filters were applied to all the physiological signals in order to eliminate baseline drift and high frequency noise. The ECG signal was filtered using a notch filter, it's a dynamic filter which can effectively attenuate the tapering regions of the signal. A $50 \mathrm{~Hz}$ notch filter was utilized for this experiment, unlike other established filter (eg: Blackman), it does not substantially reduce the oscillations of the signal, it does not attenuate the signal further above or below the required cut-off frequency which often leads to a much steeper signal than wanted [86]. The signal was smoothed using wavelet decomposition method using wavedec (db6) with

order 10, which minimizes the amount of signal attenuated after filtering the noise associated due baseline wander, power line interference and motion artifacts (a process diagram for preprocessing ECG signal is displayed below on Figure 15). GSR signal was preprocessed using 
a low pass Butterworth filter with an order of 6 and cut-off frequency of $0.5 \mathrm{~Hz}$ (a process diagram for preprocessing GSR signal is displayed on Figure 16). Respiration signal was very noisy and was band pass filtered at cut-off frequencies $0.5-35 \mathrm{~Hz}$ using a Blackman filter, which is a point specific filter (a process flow chart used to describe the preprocessing process for respiration signal is displayed below on Figure 15). The signal was sampled at $32 \mathrm{~Hz}$.

Interpolation was utilized to achieve a more efficient PSD of the ECG signal in frequency domain, it is the process of up sampling (introducing zero values to create a longer signal) followed by filtering (removes unwanted spectral images) [88]. It creates a sample which is inserted in-between the original signal (efficient estimation of an unknown/messy information which is coherent with the rest of the original signal) in order to delineate a more efficient/smoother PSD.

\subsection{Method/Implementation}

\subsubsection{Subjects}

15 subjects were analyzed for the study between the ages of 18-65, specific subjects were not recruited for the experiment, which included IT personnel, Post-docs, university administrators, university students. The subjects did not suffer from any psychological or physical pathology. There wasn't any limitations to their stress level prior to the experiment, it was not conducted during a specific time, subjects arrived before/after work, after meetings, during lunch time, sometimes they were stressed after a busy day and some of them were relaxed since its summer for most students. However, caffeine and tobacco intake along with strenuous exercise 1 hour prior to the experiment was avoided. All subjects had to sign a consent form prior to the start of the experiment, they had to wear the captive ECG, GSR and Respiration sensor throughout the experiment and avoid excessive movement to minimize noise (minimal movement was required to transition from one phase of the experiment to the other).

\subsubsection{Protocol}

The duration of the experiment was 42 minutes, it involved 4 tasks and meditation in between. 


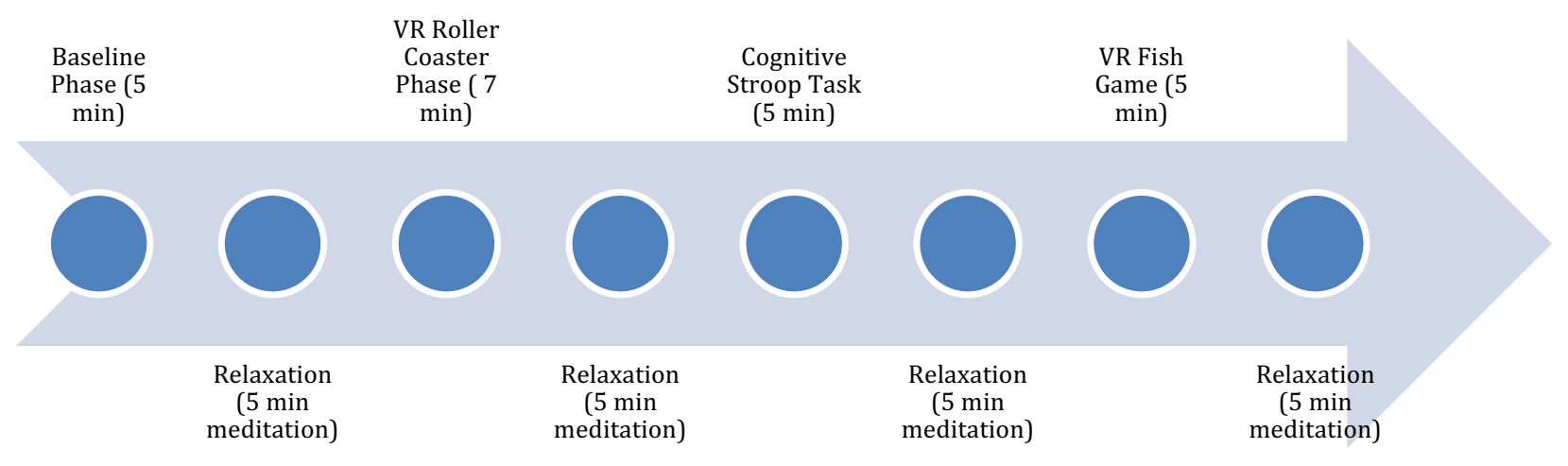

Figure 13: Protocol for data collection from the four distinct phases: Baseline phase, VR Roller Coaster Phase, Cognitive Stroop Task and VR Fish Game

Task 1: Baseline Measurements

Initially all subjects had to de-excite and return to a relaxed state by meditating for 3-5 minutes. For task 1 they were required to sit at a table for 5 minutes in order to detect their baseline measurements, they were asked to limit their body movements as much as possible, but they were allowed to browse or read during this period.

Task 2: VR Roller Coaster Simulation

During this procedure all subjects had to wear a VR headset in order to engage in a VR simulation of a roller coaster ride, it lasted for about 8 minutes and the purpose of the study was to induce stress, anxiety and increase the HR of the subject. The headset was placed from the back of the head to the front, the straps on the left, right and top were initially to place the headset properly, it was tightened to minimize blur, lens below the right eye were adjusted in order to minimize any blur along the vertical edges. Following task 2, subjects were instructed to meditate for another 3-5 minutes following a guided meditation video on YouTube. 
Task 3: Cognitive test on the computer

During this phase subjects were required to play a cognitive game for about 5 minutes, if they were able to reach the score of 50 before the end of the 5 minutes duration then that would mark the end of this phase, however the incentive behind this task was to induce stress, reaching a score of 50 is impossible and it existed to increase anxiety. Subjects had to mediate for another 3-5 minutes to de-excite themselves.

\section{Task 4: Bubble Bloom}

The VR head was utilized during this phase to allow the subjects to play a bubble game, they had to shoot bubbles using a controller to capture fish. This game is very relaxing and easy, it was implemented inorder to reduce stress/anxiety within people. 


\subsubsection{Data Collection}

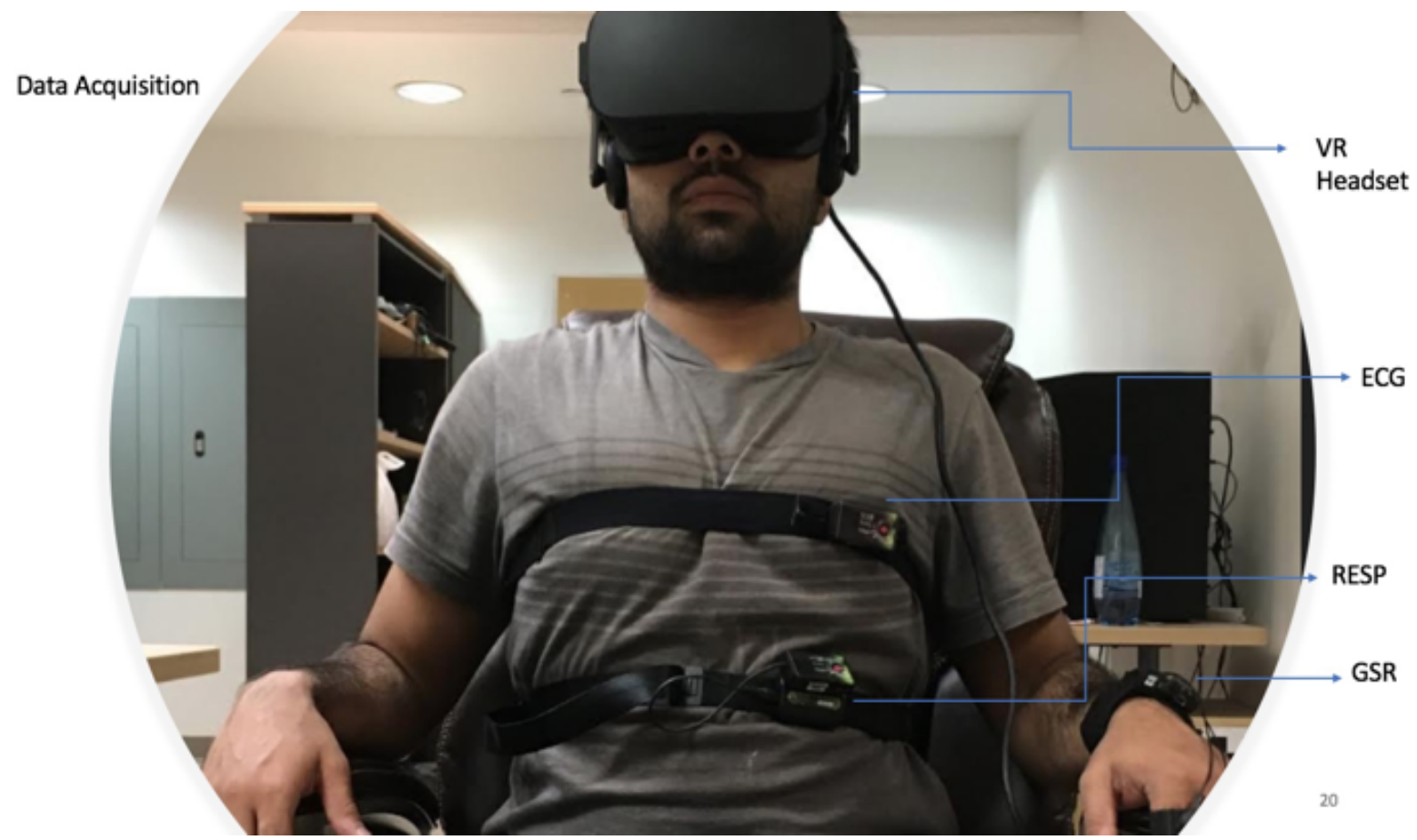

Figure 14: Data acquisition through wireless ECG, GSR and RESP devices

Captiv biosensors were used for data acquisition, three other sensors were used to capture

physiological signals associated with various activities involved within the experiment. The three sensors include: T-Sens ECG, T-Sens Respiration, T-Sens Skin Conductance. T-Sens ECG was utilized to measure the raw ECG signal (in $\mathscr{M} \mathrm{V}$ ), it's a belt embedded with electrodes, placed on the thorax and has direct contact with the skin in order to receive the most accurate signal. T-sens Respiration is also a belt embedded with a respiration sensor (electrodes are attached to the belt), the belt is attached to the thorax or abdomen (abdomen for this experiment since ECG sensor was in direct contact with the thorax), it does not require direct contact with the skin. It is used to measure abdominal and thoracic movements in order to analyze respiration rhythm and amplitude. In order to acquire GSR signal T-sens skin conductance sensor was utilized, it's a wristband with the sensor embedded in the middle along with two electrodes which is placed on two fingertips on the subjects non dominant hand, the electrodes are in direct contact with the skin. It is used to measure stress through skin conductance which represents the variation of EDA (electrodermal activity) associated with sweat activity. 


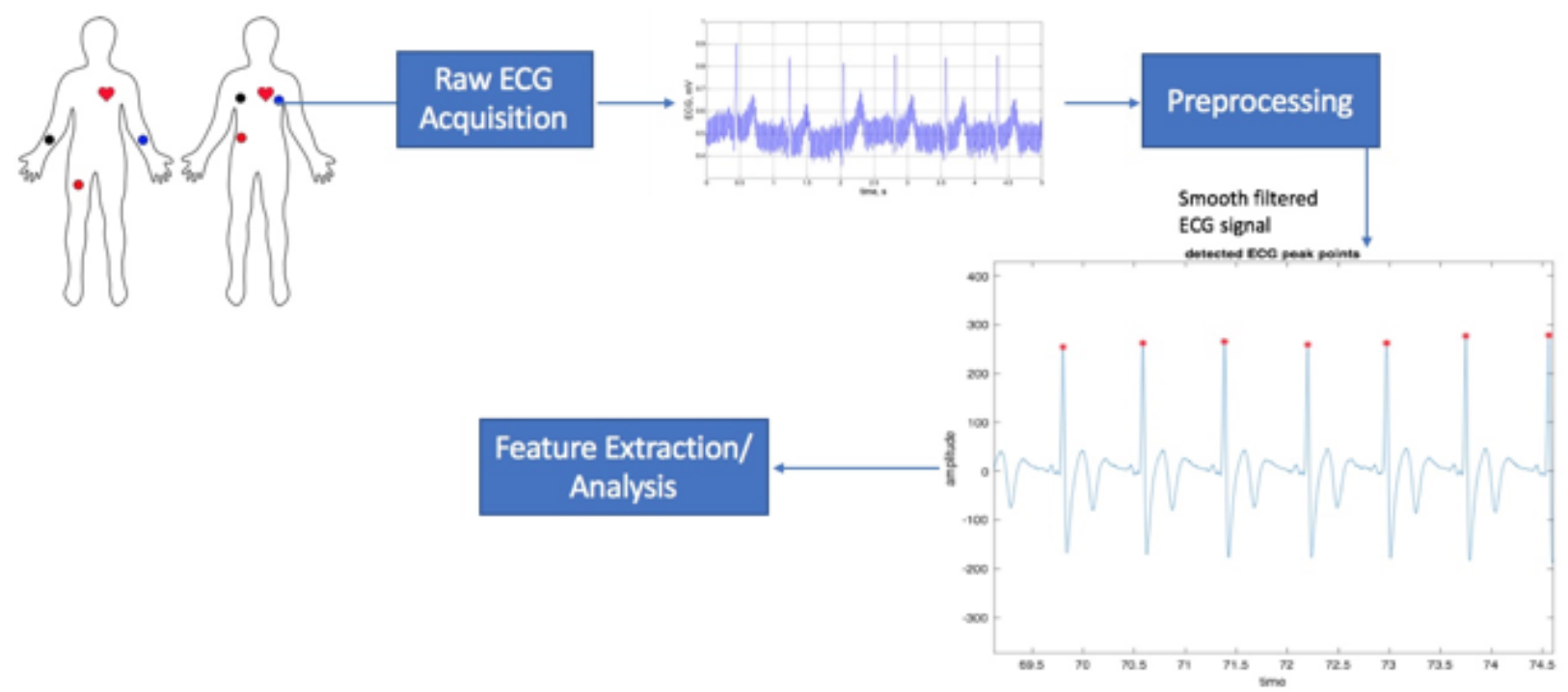

Figure 15: Notch Filter and 1D - Wavelet Decomposition Process Flow Chart for preprocessing ECG signal (in preprocessing write a sentence to look here.

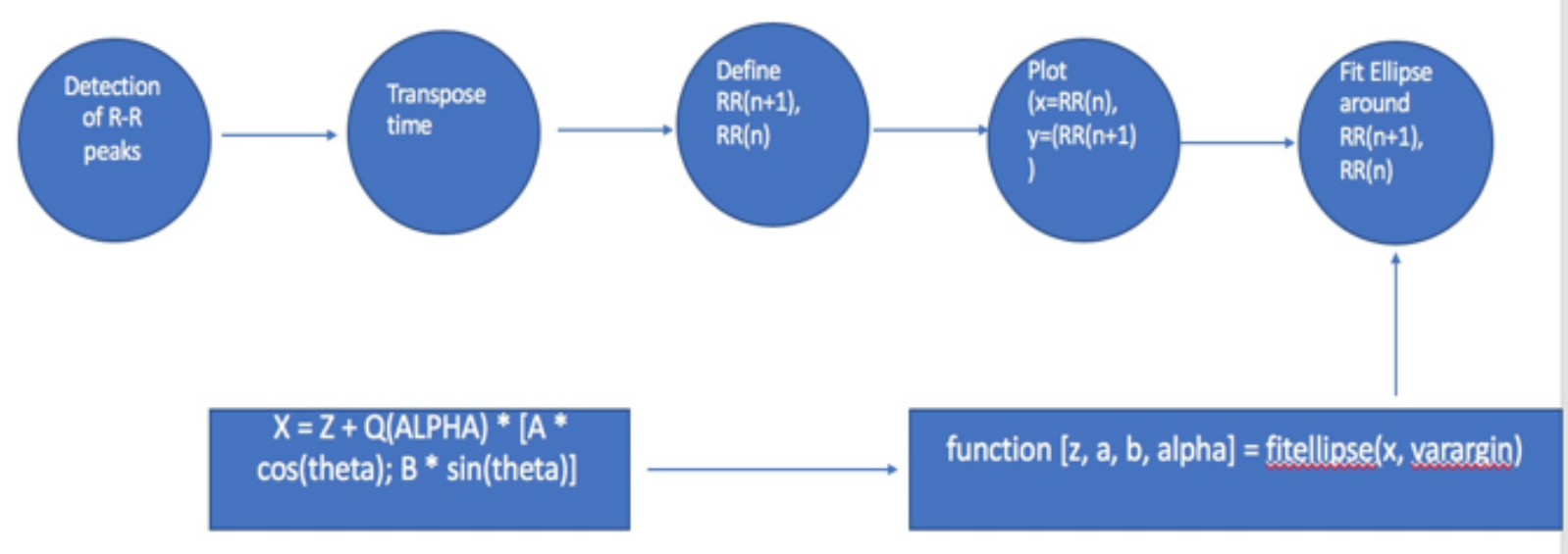

Figure 16: Process Flow Chart for plotting a poincare plot 

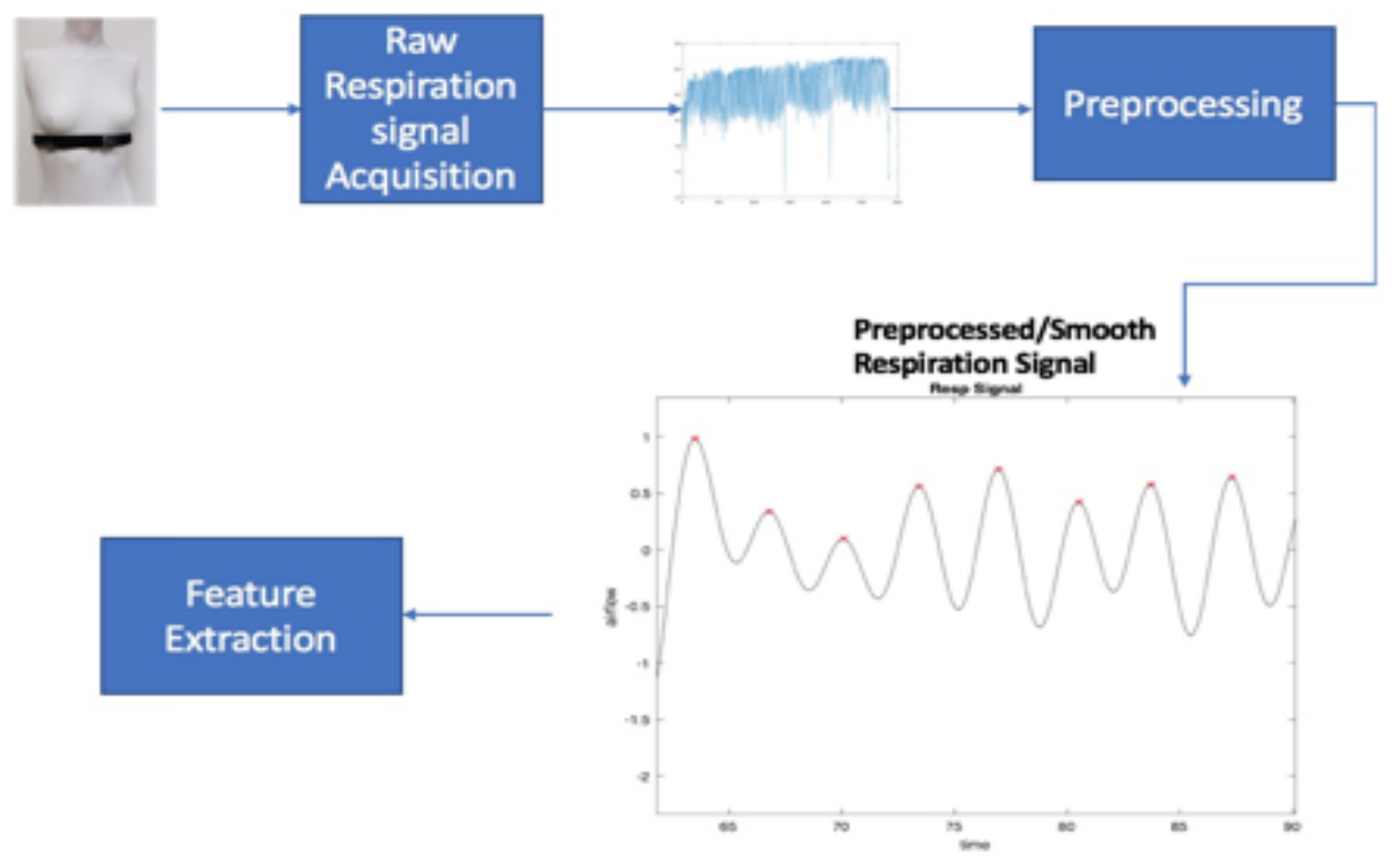

Figure 17: Blackman Filter and 1D - Wavelet Decomposition Process Flow Chart for preprocessing Respiration/EDR signal.

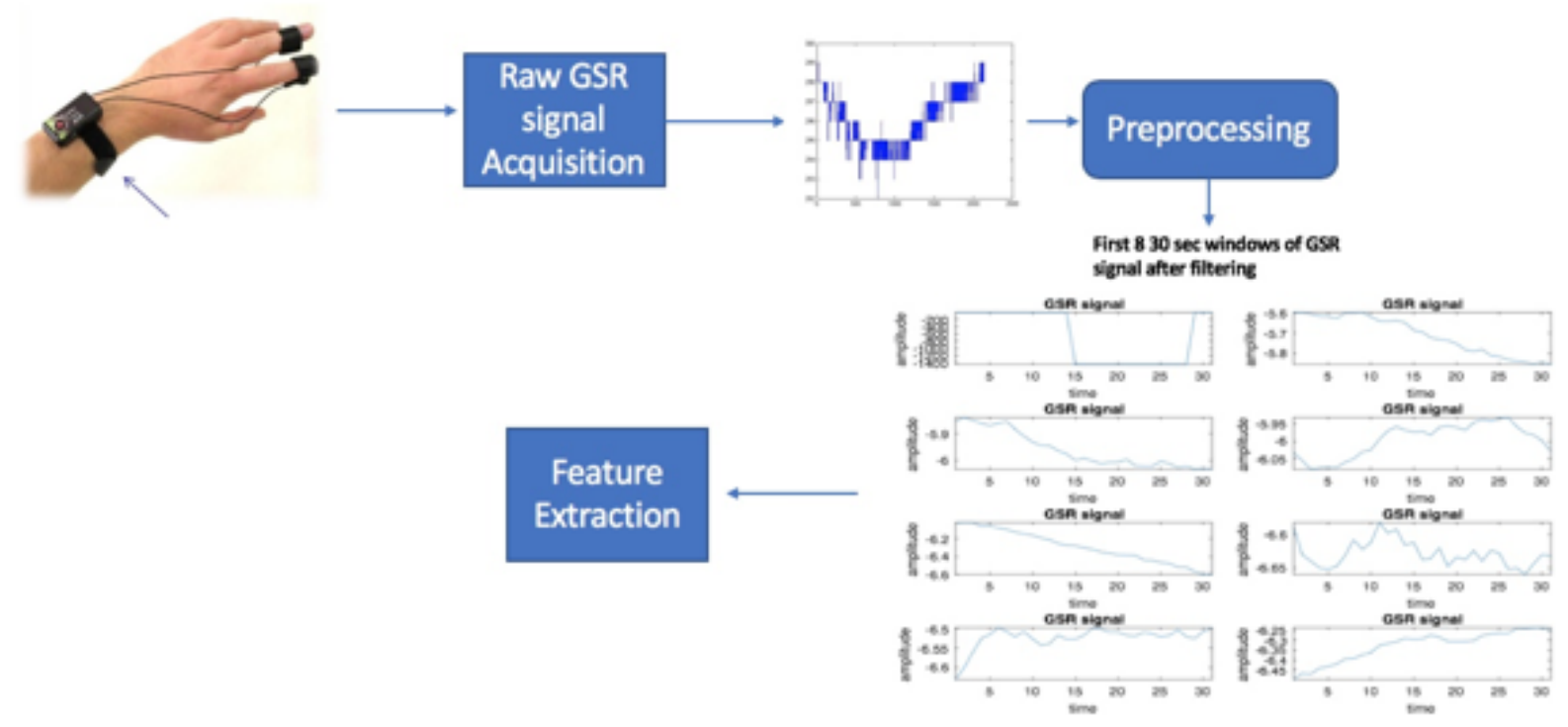

Figure 18: Low Pass Butterworth Filter and sliding 30 second windows process flow chart for GSR/EDT signal. 


\begin{tabular}{|c|c|c|c|c|c|}
\hline Data Acqusition & Preprocessing & HRV Computation & $\begin{array}{l}\text { Feature Extraction } \\
\text { (Time Domain) }\end{array}$ & $\begin{array}{l}\text { Feature Extraction } \\
\text { (Frequency } \\
\text { Domain) }\end{array}$ & Stress Assessment \\
\hline $\begin{array}{l}\text { T-Sens ECG sensor } \\
\text { T-Sens Skin } \\
\text { Conductance (GSR) } \\
\text { sensor } \\
\text { T-Sens Respiration } \\
\text { sensor }\end{array}$ & $\begin{array}{l}\text { Notch Filter (Time } \\
\text { domain) } \\
\text { Wavelet Decomposition } \\
\text { (smoothing Time } \\
\text { domain) } \\
\text { Interpolation } \\
\text { (Frequency dom.) } \\
\text { Low Pass Butterworth } \\
\text { Filter(GSR) }\end{array}$ & $\begin{array}{l}\text { R-R peak Detection/R-R } \\
\text { interval variation } \\
\text { Mean HR Variation }\end{array}$ & $\begin{array}{l}\text { R.Peak Detection } \\
\text { Poincare plot } \\
\text { Mean HR } \\
\text { RMSSD } \\
\text { PNN50 } \\
\text { SDNN } \\
\text { SD1 } \\
\text { SD2 } \\
\text { ADEN } \\
\text { GSR } \\
\text { RESP }\end{array}$ & $\begin{array}{l}\text { Interpolation } \\
\text { AR (pyulear) Method } \\
\text { Lomb Scargle } \\
\text { Periodogram (plomb) } \\
\text { VLF } \\
\text { LF } \\
\text { HF } \\
\text { LF/HF } \\
\text { TP }\end{array}$ & $\begin{array}{l}\text { Variation in Time } \\
\text { Domain Parameters } \\
\text { Variation in Frequency } \\
\text { domain Parameters } \\
\text { Increased LF, LF/HF or } \\
\text { Decreased HF (Stress) } \\
\text { vice versa relaxed if } \\
\text { those parameter follow } \\
\text { opposite pattern } \\
\text { Increased HR or } \\
\text { decreased HR } \\
\text { etc }\end{array}$ \\
\hline
\end{tabular}

Figure 19: Design process used to analyze HRV in time and frequency domain

Filter Data using Band pass Notch Filter $(50 \mathrm{~Hz})$ and smooth ECG signal using order 10 Wavelet Decomposition Method (wavedec, 'db6)

R-Peak Detection using MATLAB

R-R variability Computation using MATLAB

SDNN, PNN50, RMSSD, SD1,SD2, ApEn, Mean HR obtained using R-R interval

Figure 20: Process behind analyzing HRV in Time Domain 


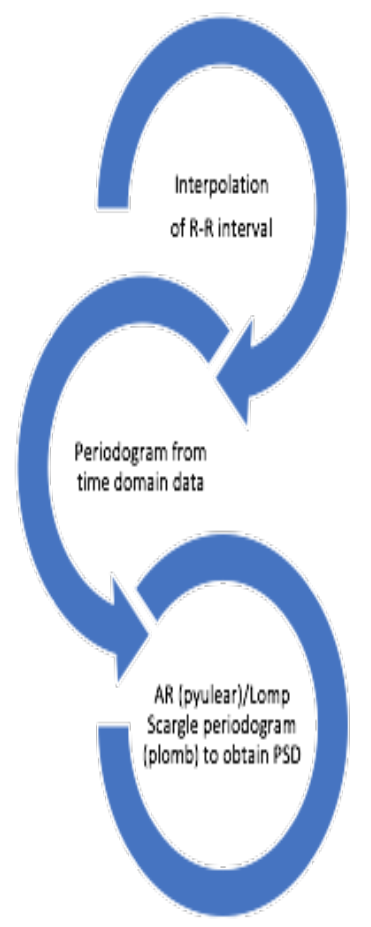

Figure 21: Process utilized to analyze HRV in Frequency Domain through MATLAB 


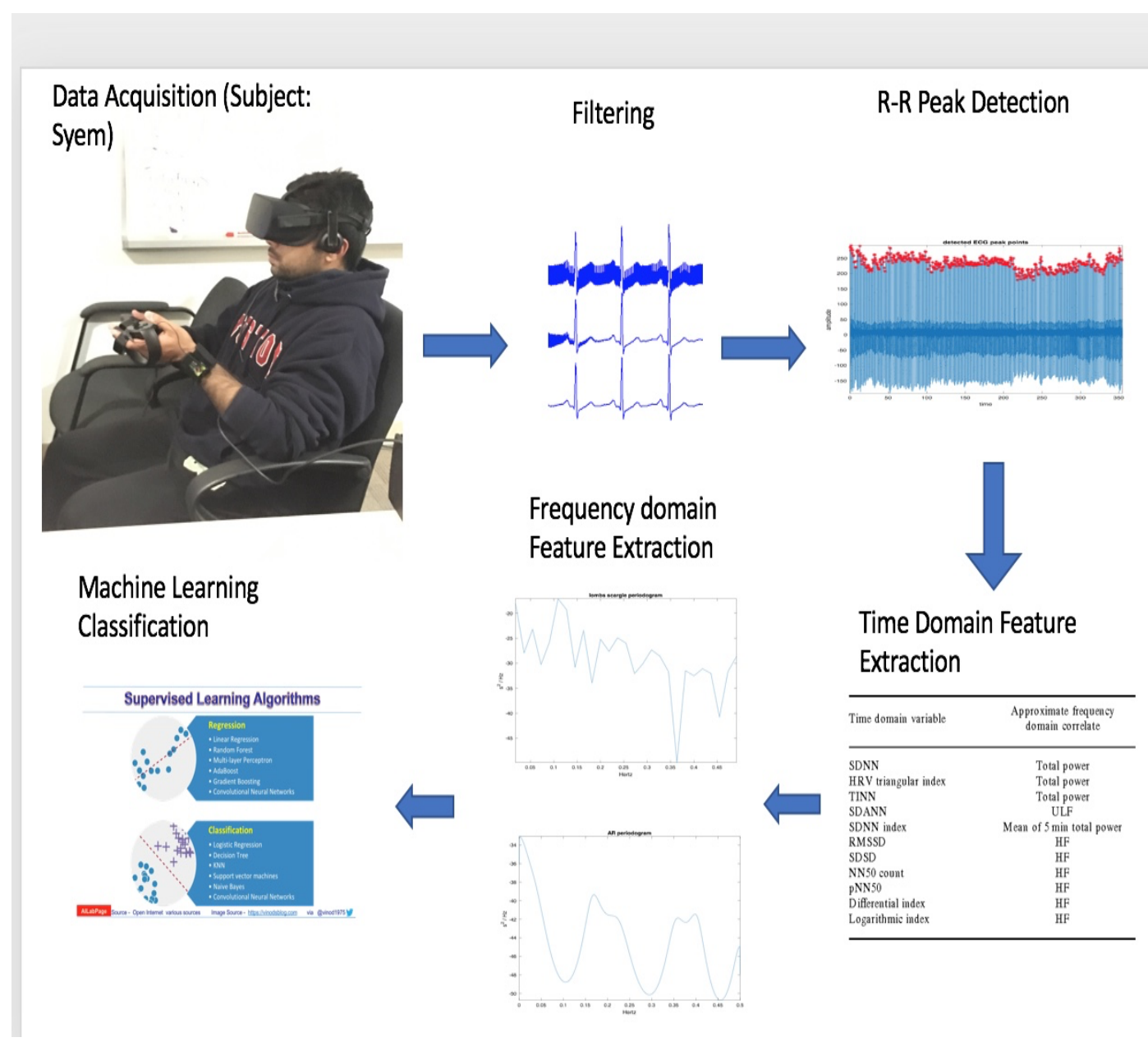

Figure 22: Visual Representation of Data Acquisition - Time/Frequency Domain Feature Extraction and Analysis - Machine Learning Classification

\subsection{Results}

In order to understand various physiological functions associated with stress, 3 physiological signals (HRV, EDA, Respiration) were detected from each subject. The experiment consisted to 4 phases (T1, T2, T3, T4), initial phase was used to measure their baseline/normal parameters, phases T2 and T3 consisted of a roller coaster VR simulation and cognitive game in order to induce stress through negative emotions and cognitive load. The last phase was the VR fishing game which was a biofeedback game to relax them and return their physiological function back to normal after inducing stress. 15 subjects were analyzed for the study, 3 different physiological signals were measured from each subject for 4 phases, which resulted in 166 signals, which is less than the expected 168 signals but one subject was too 
stressed to carry on with the roller coaster phase and another signal was not detected due to technical error associated with wireless transmission of the signal data via Bluetooth. Some of the sampled data were abnormally large and inaccurate as a result of excessive noise associated with the signal. From the 166 signals, only 150 were used to extract the features and develop the final data. There were 11 complete experiments which consisted of 4 phases and 2 experiments resulted in signals for only the first three phases (the last phase signal data was not recorded). Overall the 50 samples consisted of 13 samples from baseline phase, 13 samples from VR roller coaster phase, 13 samples from Cognitive color stroop task phase and 11 samples from VR video game phase. Each sample data consisted of 21 features. The rest of the data were not detected properly, too noisy and inaccurate. Time domain variables were measured from ECG parameters, $R-R$ variation for one R-interval to the next was utilized to assess $R-R$ variability, HRV, nonlinear $S D 1, S D 2, A p E n$ and Poincare plot. The time domain $R-R$ interval variation was interpolated (cubic interpolation) to smoothen the signal, fit estimates for errors associated, provide an evenly sampled signal and transformed into power spectral density through AR (pyulear) method and Lomb scargle periodogram (plomb) to delineate frequency domain variables associated with HRV. There were 8 time domain variables in total which included $H R$, $S D N N$, RMSSD, NN50, $p N N 50, S D 1, S D 2$ and ApEn. The frequency domain parameters used to analyze HRV consisted of $V L F, L F, H F, L F / H F$ ratio and $T P$ obtained from AR and Lomb Periodogram PSD. These features were all obtained from the ECG signals. GSR_mean and GSR_std was also extracted from GSR signal in order to better analyze stress. Respiration rate was extracted from the respiration signal. In order to discriminate between stressed and relaxed subject, their physiological parameter was compared with their baseline data. An increased $L F$, decreased $H F$, increased HR, increased $L F / H F$, reduced HRV and decreased $P N N 50$, . was associated with an increase in stress levels. Respiration signal was processed through a notch filter and an increase in breathing rate was associated with an increase in stress levels. GSR was filtered using a LPF and the variations were analyzed using 30 second windows. It is used to indicate EDA activity, which measures the dynamic skin temperature associated with sweating and an increased GSR indicated an increase in stress levels. 


\subsubsection{Baseline Phase}

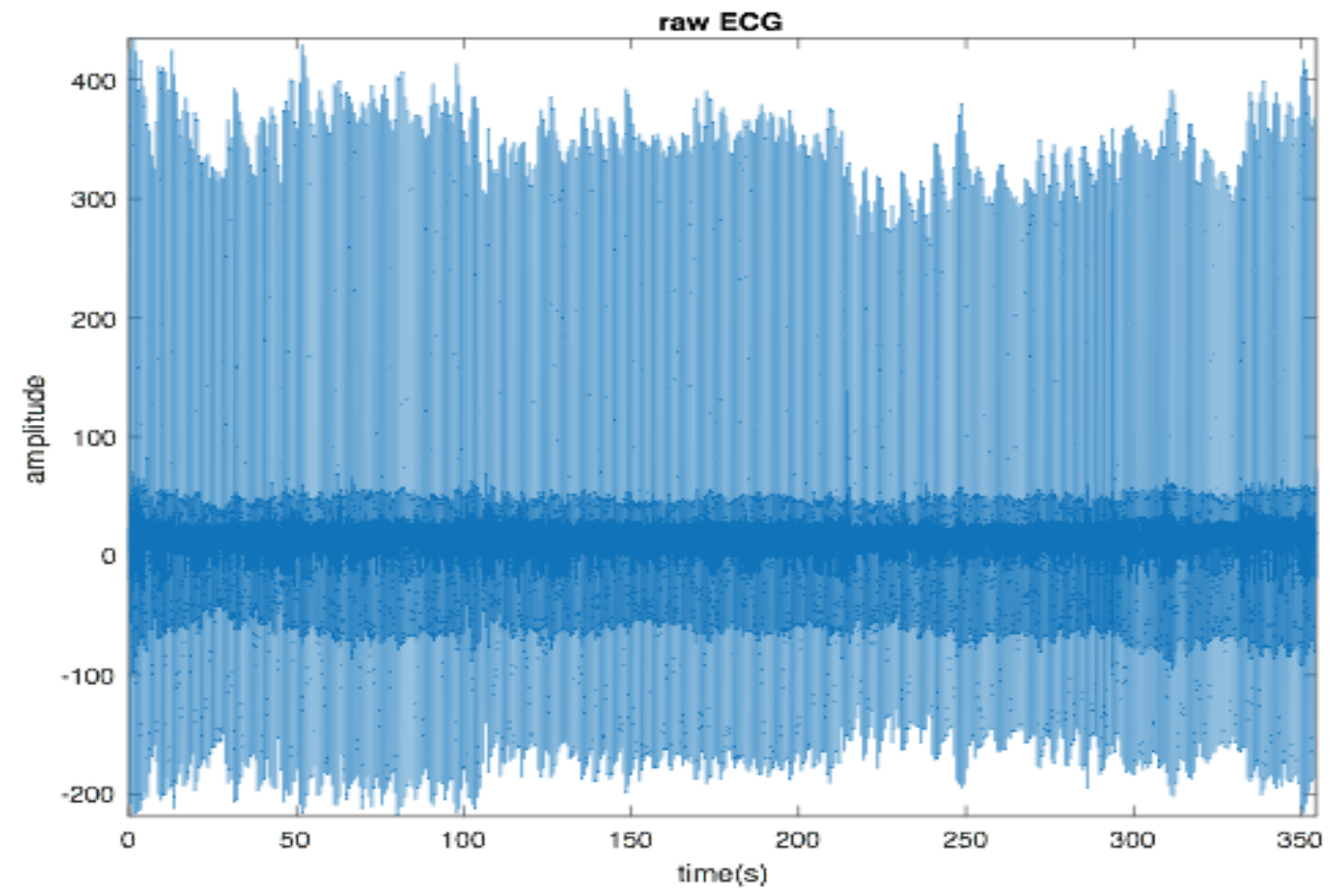

Figure 23: Sample raw ECG of a subject during baseline phase

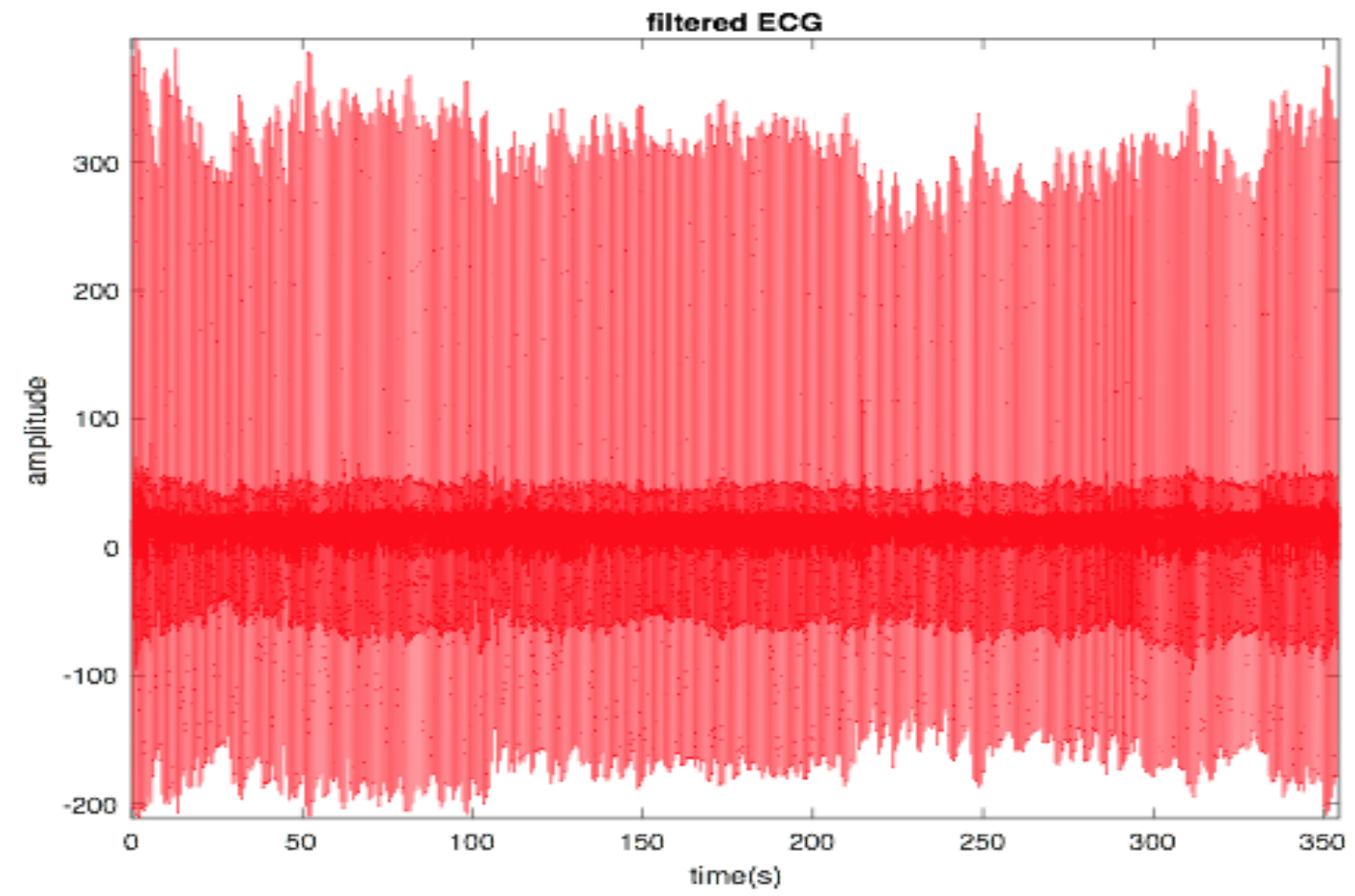


Figure 24: Filtered ECG using a notch filter, during the baseline phase

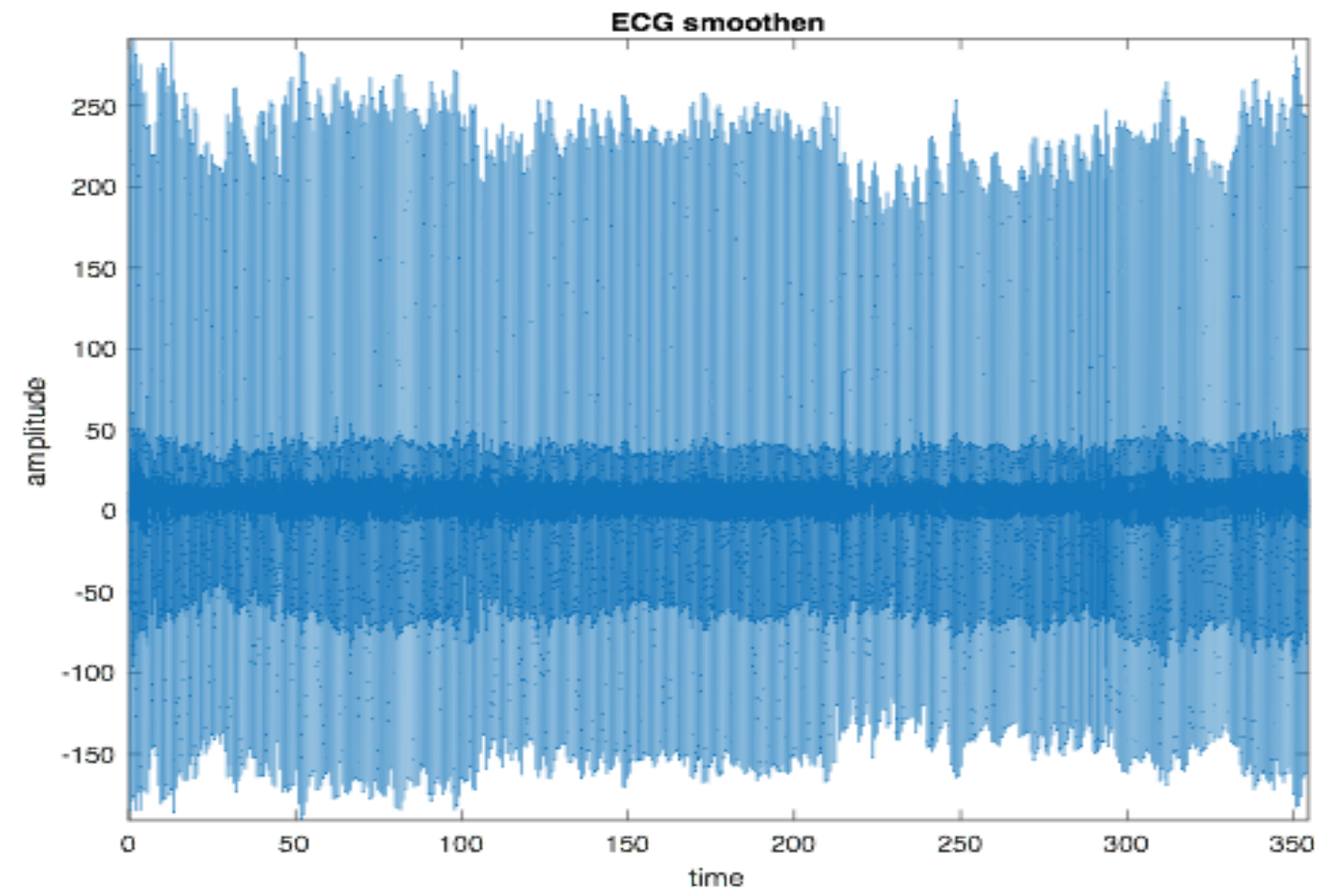

Figure 25: The signal is further smoothened through wavelet transform to remove tapering noise, and make the peaks sharper 


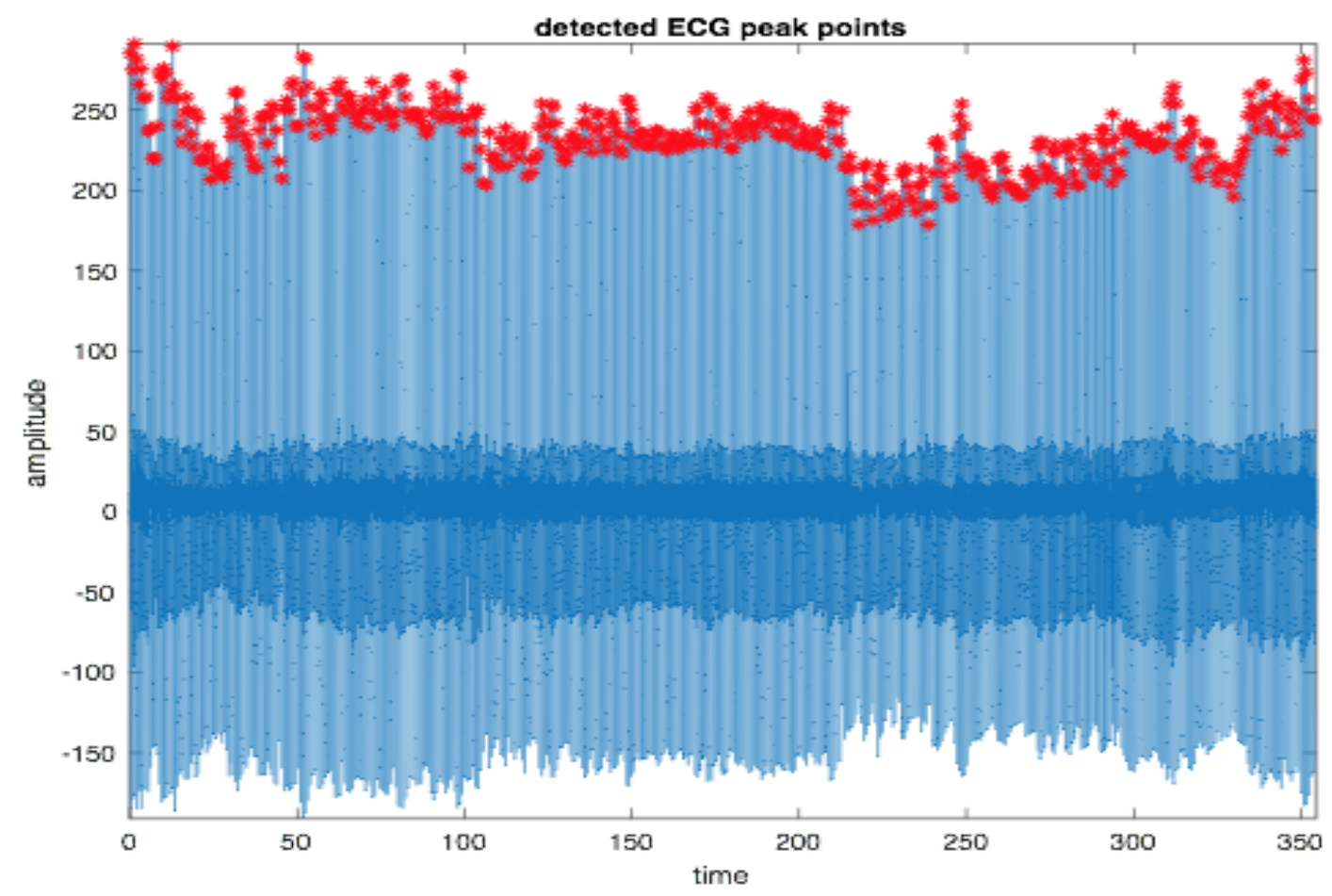

Figure 26: Detected R-peaks during the baseline phase.

The list of Figures (23-26) displayed above present the result of user 2 during the subject's baseline trial from the experiment. The signal was sampled at $250 \mathrm{~Hz}$, the duration of the phase was 5 minutes long. The ECG sensors were attached directly to the subject's thorax in order to retrieve the most accurate signal and the raw ECG Figure (Figure 21) indicates that the raw signal acquired from the subject was fairly smooth and less noisy than typical ECG signal obtained during an experiment. The purpose of the baseline task was to detect the normal physiological function of the subject before stress was induced, user2 had a mean heart rate of $84.7597 \mathrm{bpm}, S D N N$ of $0.053441, R M S S D$ of $0.031241, N N 50$ of 21 which indicates that $21 R-R$ interval had a distance between them which was greater than $50 \mathrm{msec}$ and his PNN50 was 0.20243 . 


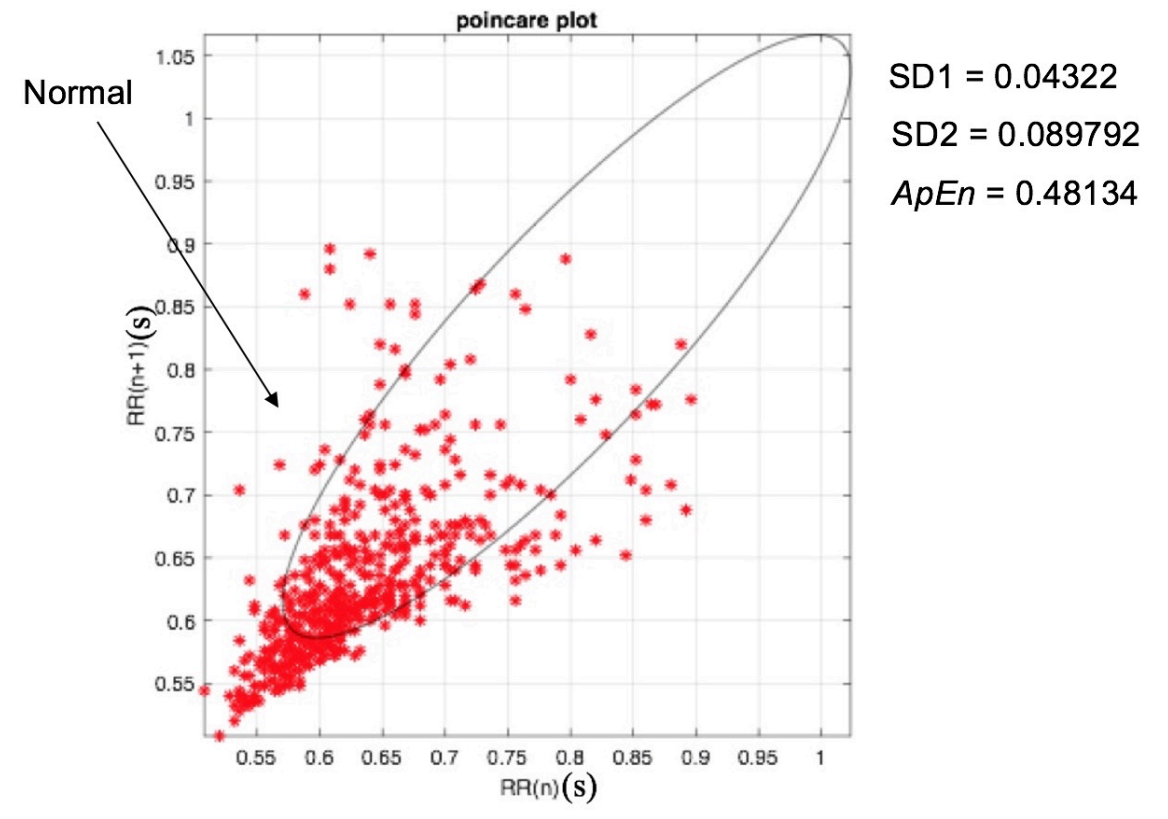

Figure 27: Poincare plot associated with baseline phase. The $R R$ intervals are measured in terms of time in seconds.

The nonlinear parameters associated with user12's baseline level are: SD1 of 0.04322 seconds, $S D 2$ of 0.089792 seconds and an approximate entropy ApEn of 0.48134. They represent the values corresponding to the Poincare plot shown in Figure 27. It represents a normal HRV, which is expected during the baseline phase. 


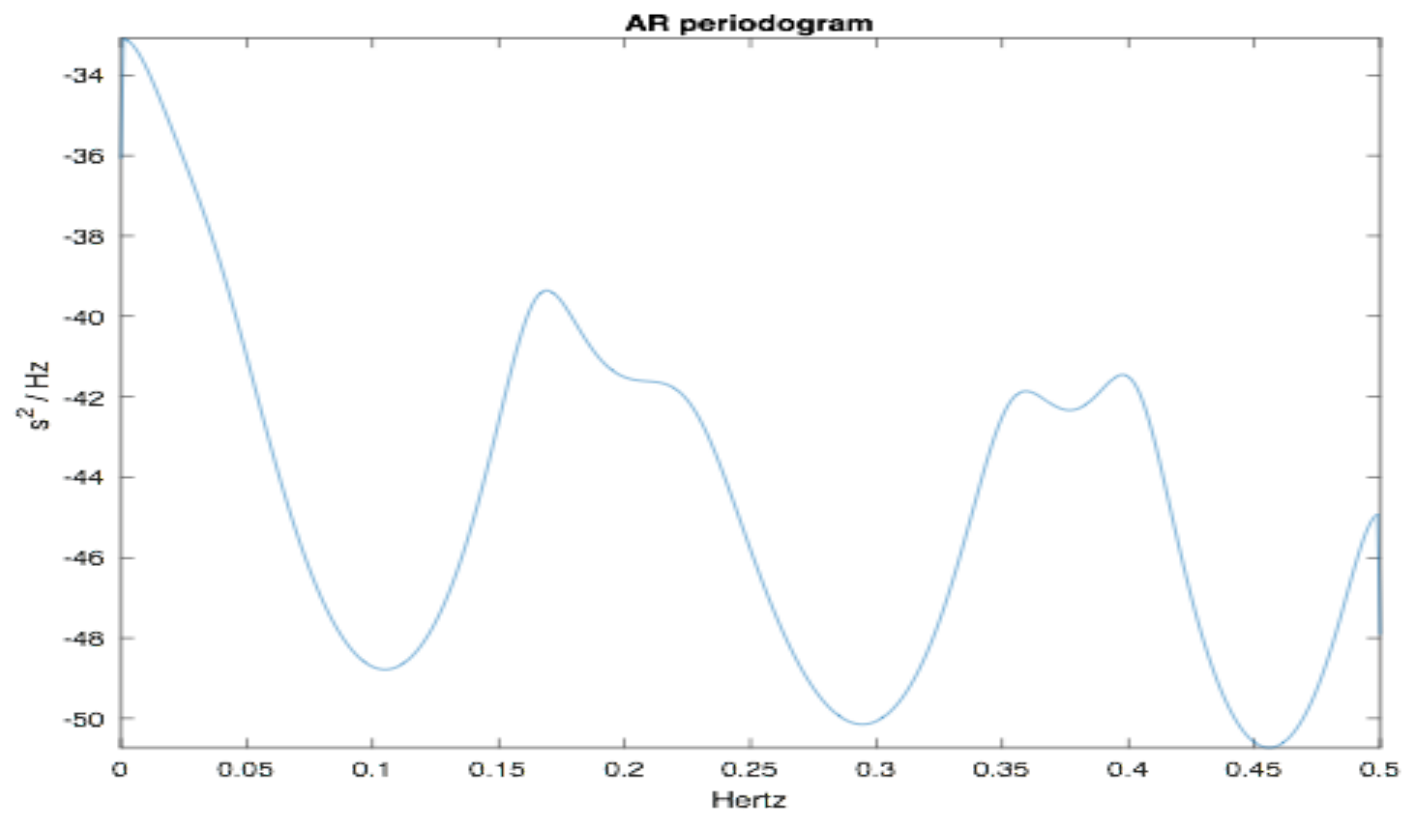

Figure 28: AR method used to delineate PSD during baseline phase.

PSD (displayed in Figure 28) is a more precise measurement of HRV, time domain parameter cannot specifically indicate the changes associated with sympathetic and parasympathetic nervous system activity. The y axis values on the graph should be disregarded, negative values only indicates that the signals actual power is less than the reference power which leads to a ratio below $1(\mathrm{ex}: L F / H F$ ratio $=0.8)$. A very low PSD of $0.4 \mathrm{~s}^{\wedge} 2$ will lead to a negative power in $\mathrm{dB}$. It just indicates that some of the PSD values are very small and have a ratio below 1 . All the PSD values obtained from $V L F, L F$ and $H F$ segments were real and positive. The $V L F$ frequency range is indicated within ranges $0.0033-0.04 \mathrm{~Hz}$ of the PSD, this band has a very strong correlation to causes of mortality, it can be utilized effectively to analyze arithmetic death and post-traumatic stress disorder,. $L F$ band (ranges between 0.04-0.15 Hz) is used to indicate SNS activity, PNS activity is assessed primarily through $\mathrm{HF}$ band (ranges between $0.15-0.4 \mathrm{~Hz}$ ) and sometimes $L F$ band. AR model used to describe the PSD associated with the baseline phase was displayed in Figure 26. User 2 results indicated a VLF of $12.4056 m s^{\wedge} 2, L F$ of $3.9326 m s^{\wedge} 2, H F$ of $12.4330 \mathrm{~ms}^{\wedge} 2, L F / H F$ ratio of 0.3163 and a total power of $30.5408 \mathrm{~ms}^{\wedge} 2$. The subject had a low $L F / H F$ ratio and $H F$ was greater than $L F$, which would indicate a fairly relaxed state to begin the experiment. 


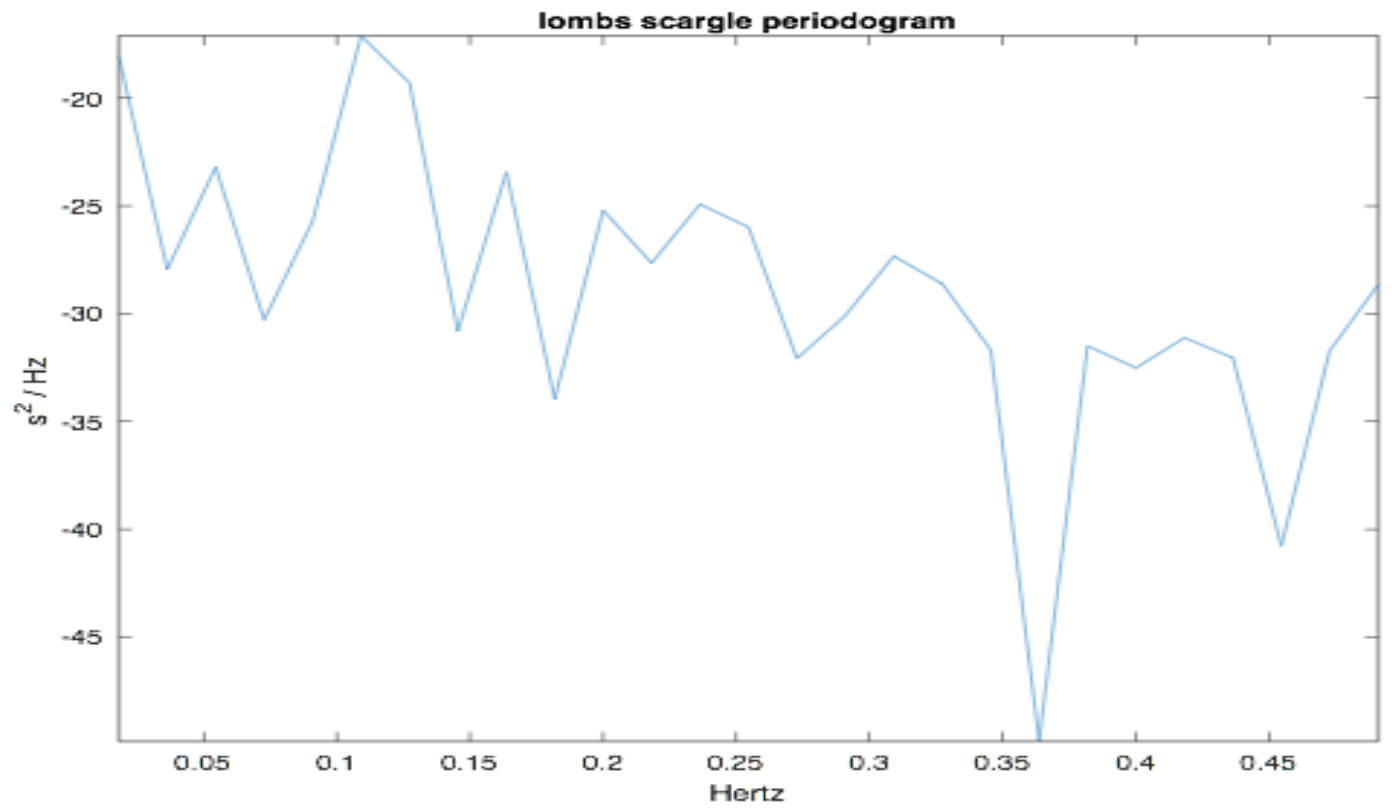

Figure 29: PSD depiction using Lomb Scargle Periodogram during Baseline phase.

Lomb Scargle periodogram which was displayed in Figure 29 resulted in different values obtained from the same subject during the baseline phase. The $y$ - axis should be disregarded, a very small PSD with a ratio below 1 (ex: $0.4 s^{\wedge} 2 L F$ value, 0.8 ratio) will have a negative power in $d B$. All the PSD values obtained from $V L F, L F$ and $H F$ segments were positive and real. The subject had a $\mathrm{VLF}$ activity of $400.2059 m \mathrm{~s}^{\wedge} 2, L F$ activity of $846.7110 m \mathrm{~s}^{\wedge} 2 H F$ activity of $432.6174 \mathrm{~ms}^{\wedge} 2$, a $L F / H F$ ratio of 1.9572 and a total power of $1.5157 \mathrm{e}+03 \mathrm{~ms}^{\wedge} 2$ these values associated with the PSD indicates that SNS activity was more dominant and the subject was awake. 


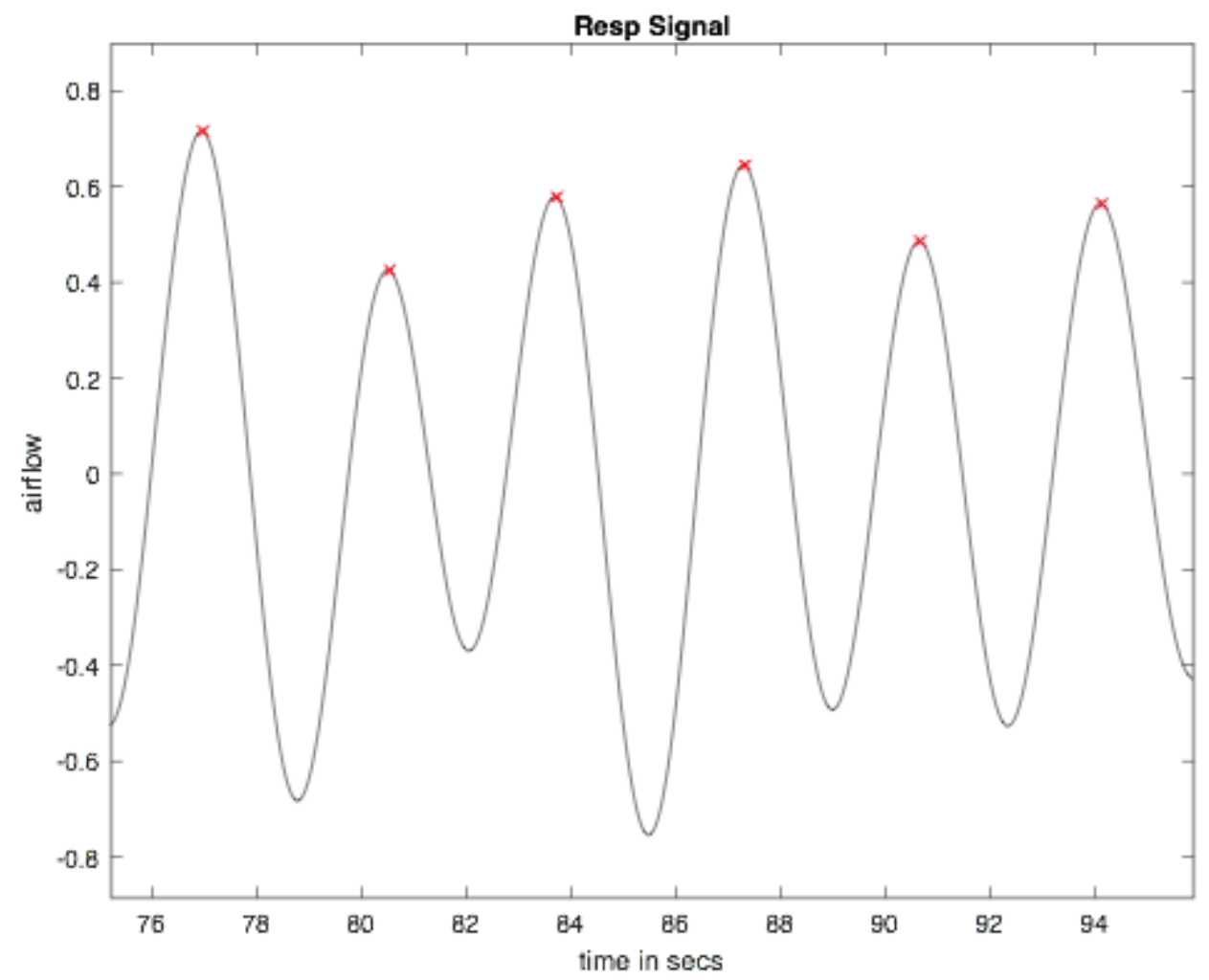

Figure 30: Respiration signal associated with baseline phase

During the Baseline phase user 12 had a breathing rate of 21.4294 breaths/min, which is around 21 breaths per minute and normal for adolescents. Respiration signal are very noisy due to motion artifacts, equipment interference and the surrounding environment. The respiration signal was displayed on Figure 30. 

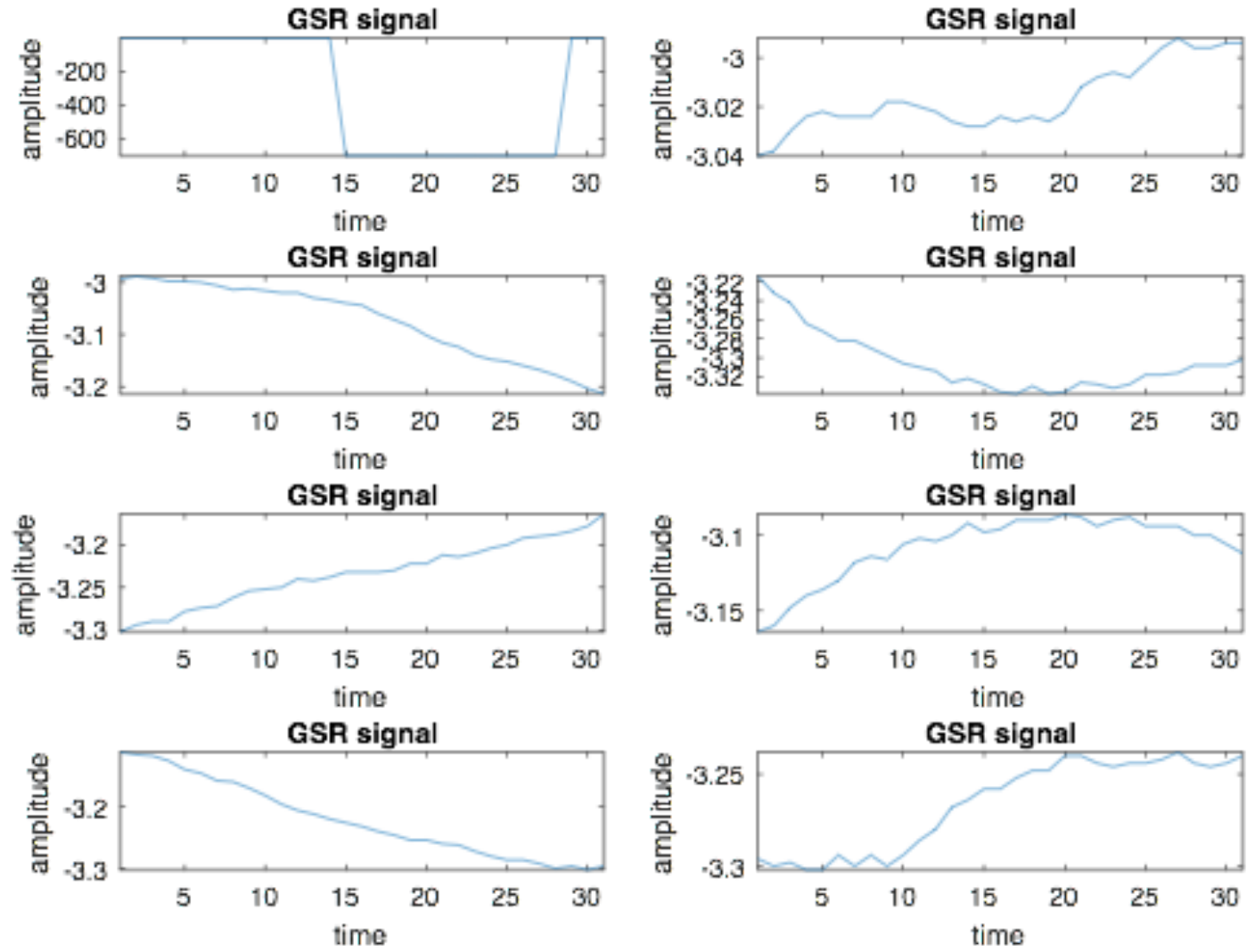

Figure 31: GSR obtained from baseline phase using 30 second window. Time in seconds.

GSR signal obtained from baseline phase (displayed in Figure 31) had an average mean of 3.175 $\mu \mathrm{S} / \mathrm{sec}$ and average std (standard deviation) of $0.0379 \mu \mathrm{S} / \mathrm{sec}$. None of the subplot genuinely depicts a graph which includes an instantaneous peak due to a stimulus associated with increased stress. The plots indicate that during the baseline phase, the subject was fairly normal $/ \mathrm{calm}$.

\subsubsection{Roller Coaster Phase}




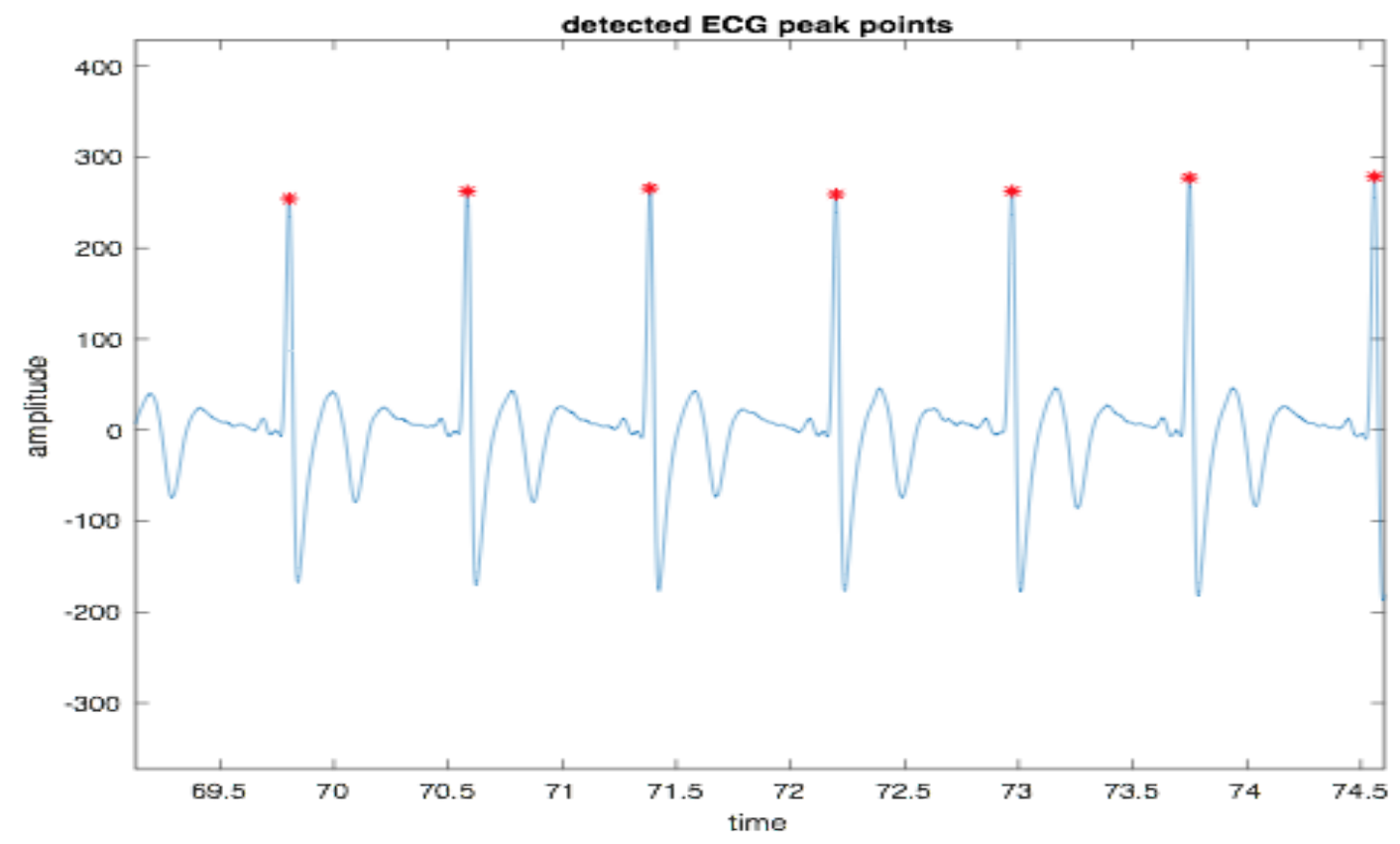

Figure 32: $R-R$ peaks detected during roller coaster phase (phase/T 2). Time in seconds

Figure 32 represents the plot of the $R-R$ peaks obtained from the roller coaster phase, the purpose of the phase was to induce stress and it was around 8 minutes long. Although user 2 did not experience an increase in HR, the subject's HR decreased to a mean HR of $73.4106 \mathrm{bpm}$. His $S D N N$ which is generally used to represent overall HRV slightly increased to 0.07269 . His RMSSD also increased to $0.083187,124 \mathrm{NN}$ interval had a distance between them which was greater than $50 \mathrm{msec}$, represented by NN50 of 124 and his pNN50 was 0.14045 which decreased from 0.20243 . PNN50 shares a strong correlation to $H F$ which decreases due to increase in stress $[81][83]$. 


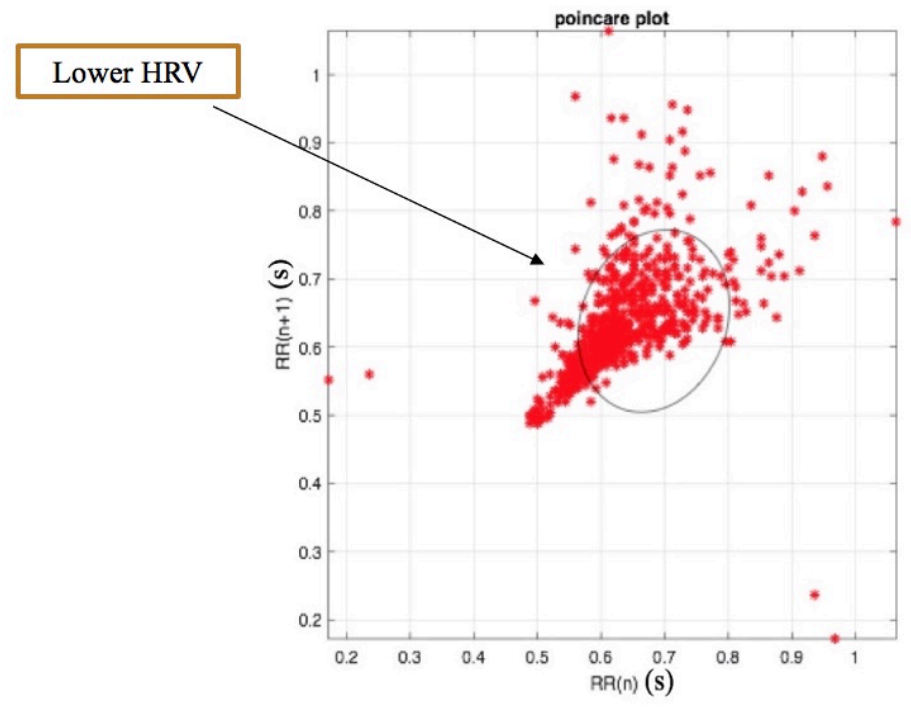

$$
\begin{aligned}
S D 1 & =0.058844 \\
S D 2 & =0.10249 \\
A p E n & =0.57412
\end{aligned}
$$

Figure 33: Poincare plot of user 12 during the roller coaster phase. $R R$ interval measured in seconds. The plot represents a HRV which is lower than previous baseline phase, indicating an increase in stress.

The Poincare plot displayed in Figure 33 consists of points within the middle-upper right corner, which often indicates a lower HRV and a stressed subject. The SDI during roller phase increased to 0.058844 seconds, SD2 slightly increased 0.10249 seconds and ApEn noticed the largest variation from the baseline phase, it increased to 0.57412. Increase in $A p E n$ indicates an increase in SNS activity, the roller coaster simulation seems to have been more stressful than the baseline phase. 


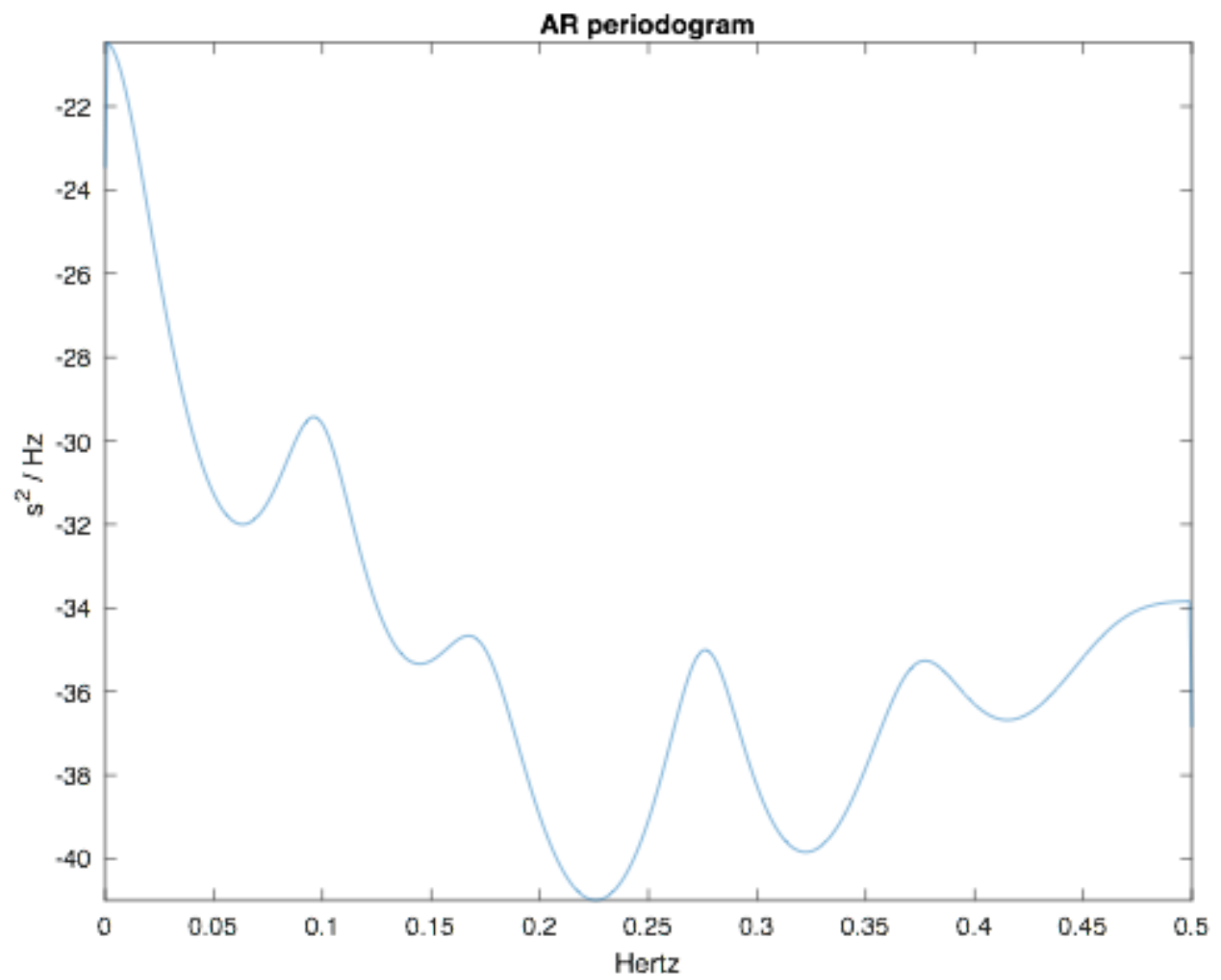

Figure 34: AR periodogram obtained from Roller coaster VR simulation.

Figure 34 describes AR periodogram obtained from user 2 during VR roller coaster simulation, the purpose was to induce stress through negative valence. The subject resulted increased values associated with each frequency band, VLF substantially increased to $170.5573 \mathrm{~ms}^{\wedge} 2, L F$ also increased to $77.1350 \mathrm{~ms}^{\wedge} 2, H F$ had noticeable increase up to $48.8597 \mathrm{~ms}^{\wedge} 2, L F / H F$ ratio also increased to 1.5787 and total power had a significant increase resulting in $324.6030 \mathrm{~ms}^{\wedge} 2$. The Roller coaster phase had a noticeable impact on the physiological function of the subject which will be discussed in the discussion section. 


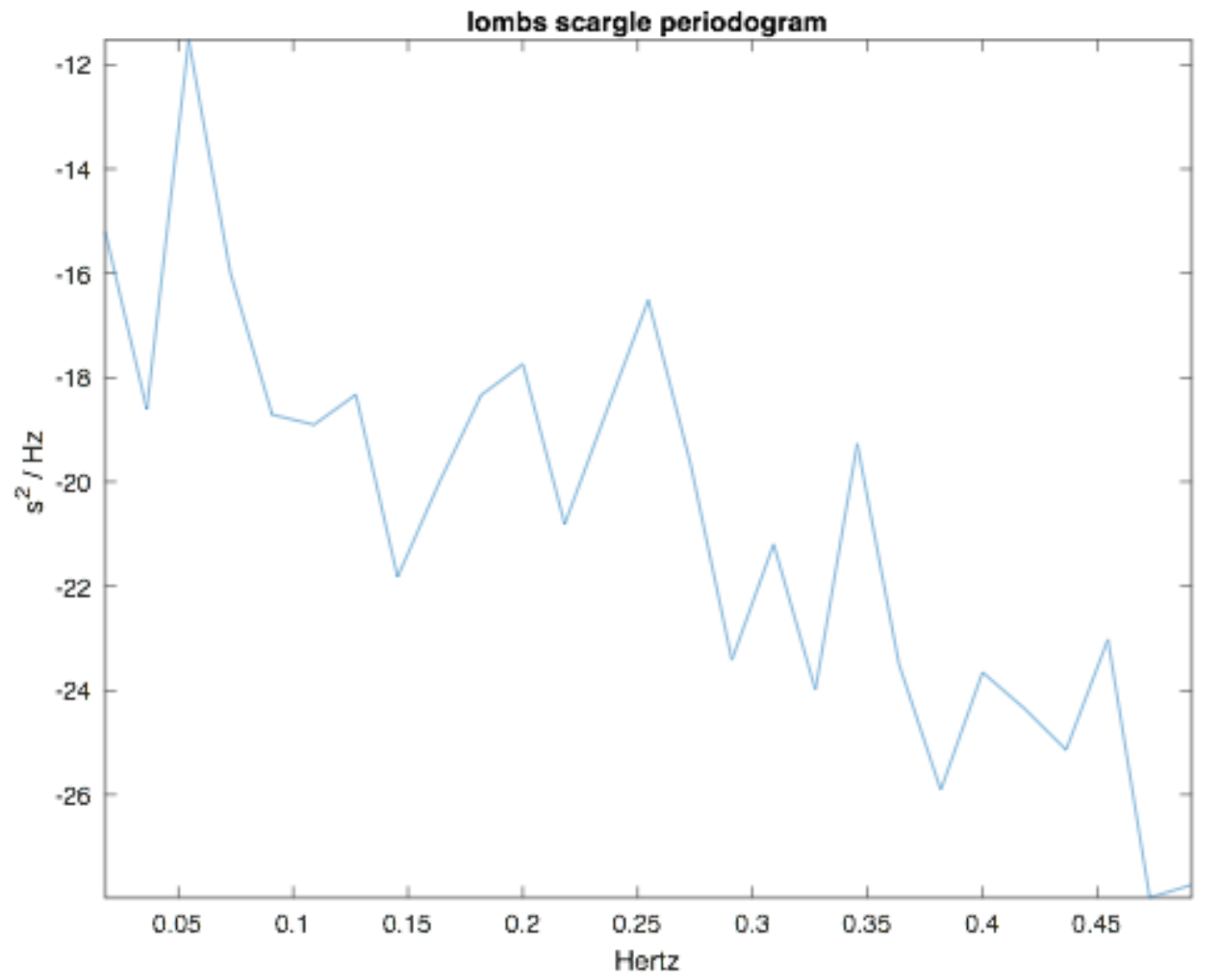

Figure 35: Lomb Scargle periodogram obtained from VR roller coaster phase

Figure 35 depicts the Lomb Scargle periodogram obtained from the VR roller coaster simulation, the results just like AR method increased for all frequency bands. Subjects $V L F$ increased to $2.0788 \mathrm{e}+03 \mathrm{~ms}^{\wedge} 2$, his $L F$ also increased to $3.0326 \mathrm{e}+03 \mathrm{~ms}^{\wedge} 2, H F$ noticed a significant increase as well, resulting in $2.6576 \mathrm{e}+03 \mathrm{~ms}^{\wedge} 2, L F / H F$ decreased slightly due to the increase of $H F$ resulting in a ratio of 1.1411 and total power increased twice as much resulting in $6.1448 \mathrm{e}+03$ $\mathrm{ms}^{\wedge} 2$. VR simulated the ANS activity of user2, results indicates that SNS activity increased during this phase and was the more dominant ANS activity. 


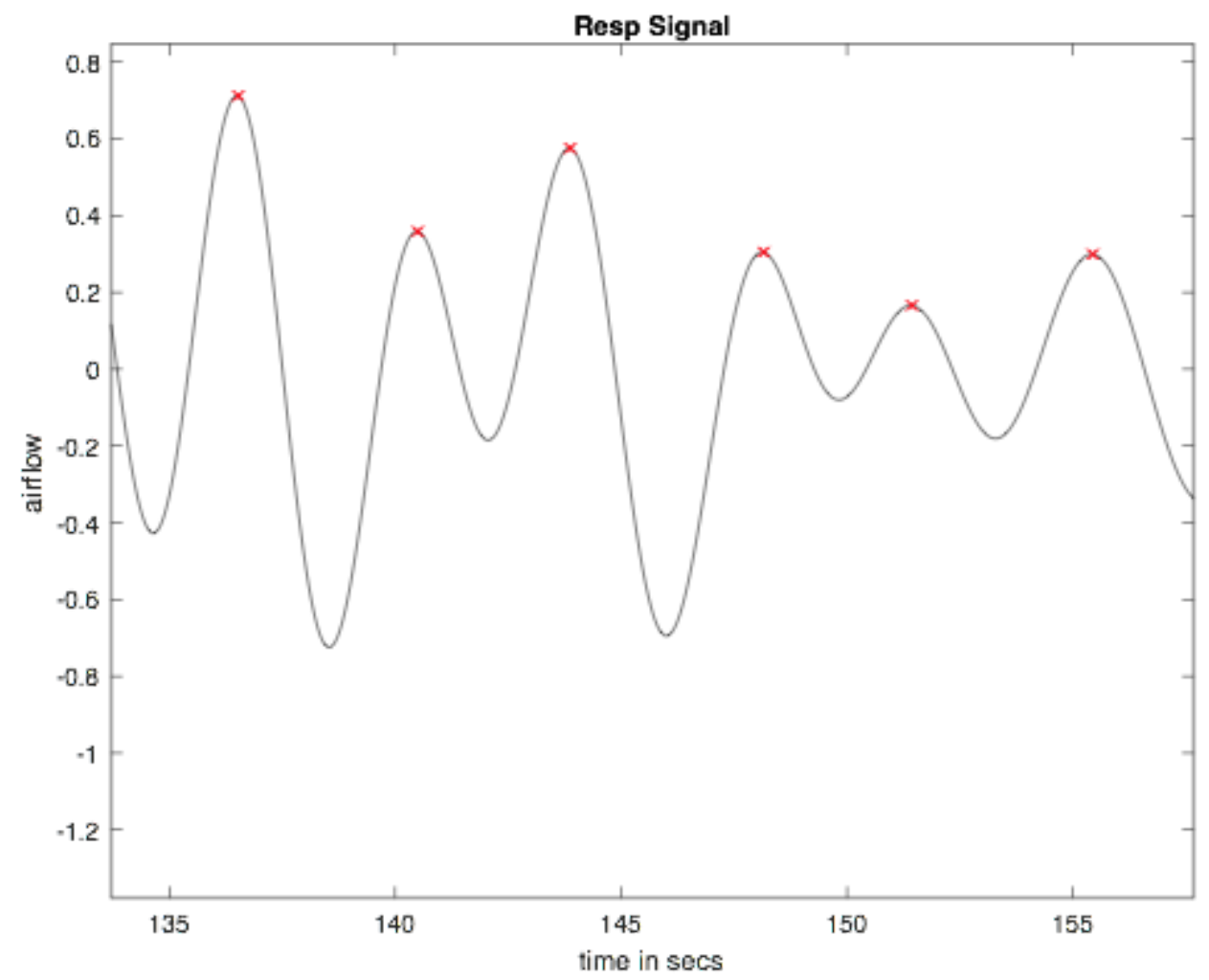

Figure 36: Respiration signal due to roller coaster phase. (x -axis indicate in secs)

The breathing rate of subject 12 increased during the roller coaster phase which is described in Figure 36. Respiration signals are often contaminated with a lot of noise due to motion artifacts, environment, even nurses often report errors when analyzing respiration rate. The subjects breathing rate increased to 24.3910 breaths/min, which is slightly higher than normal. 

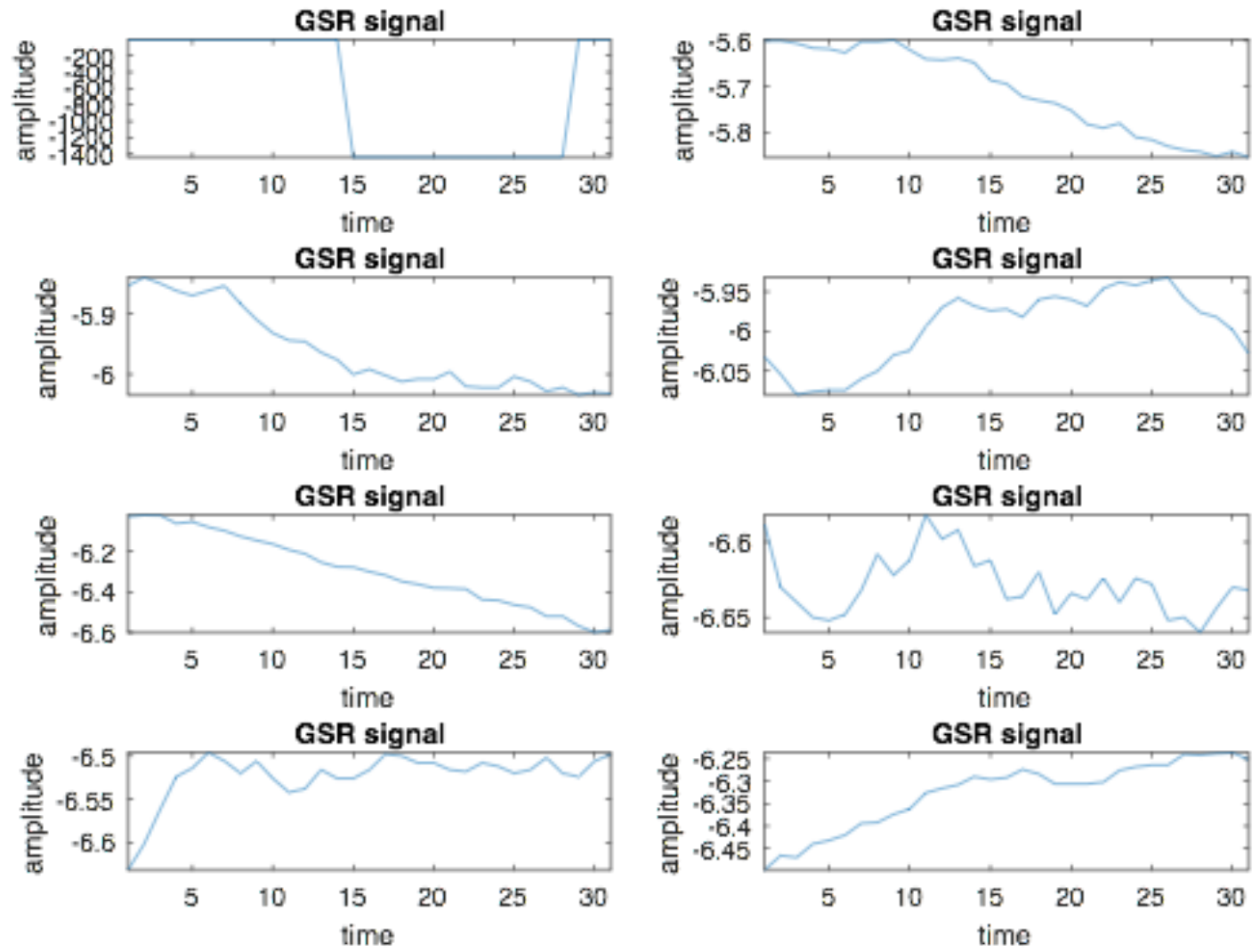

Figure 37: GSR signals associated with Roller coaster phase. Time in seconds

Figure 37 delineates the GSR signal associated with VR roller coaster phase. The subject's results indicate a mean GSR of $6.2054 \mu \mathrm{S} / \mathrm{sec}$ and GSR std of $0.07399 \mu \mathrm{S} / \mathrm{sec}$. Plot 4 has an instantaneous spike at 15 seconds, 25 seconds which can indicate stress stimulus during the 105 seconds mark and 115 seconds mark. Plot 6 has a stimulus at 11 seconds, which depicts acute stress stimulus during the 161 seconds mark (2:41 min). 


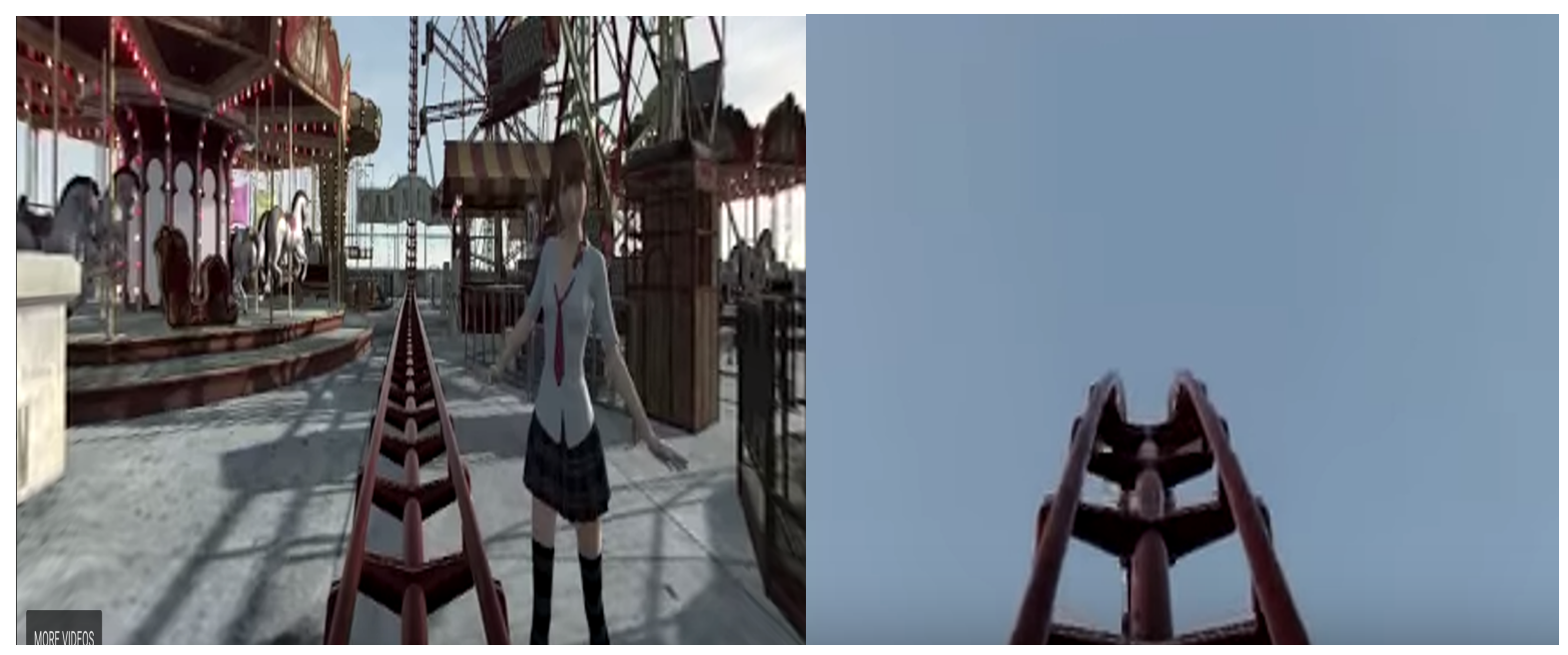
a) No stress
b) Stressed

Figure 38: Visual representation of a stress and no stress phase from VR roller coaster simulation.

\subsubsection{Cognitive Color Stroop Test}

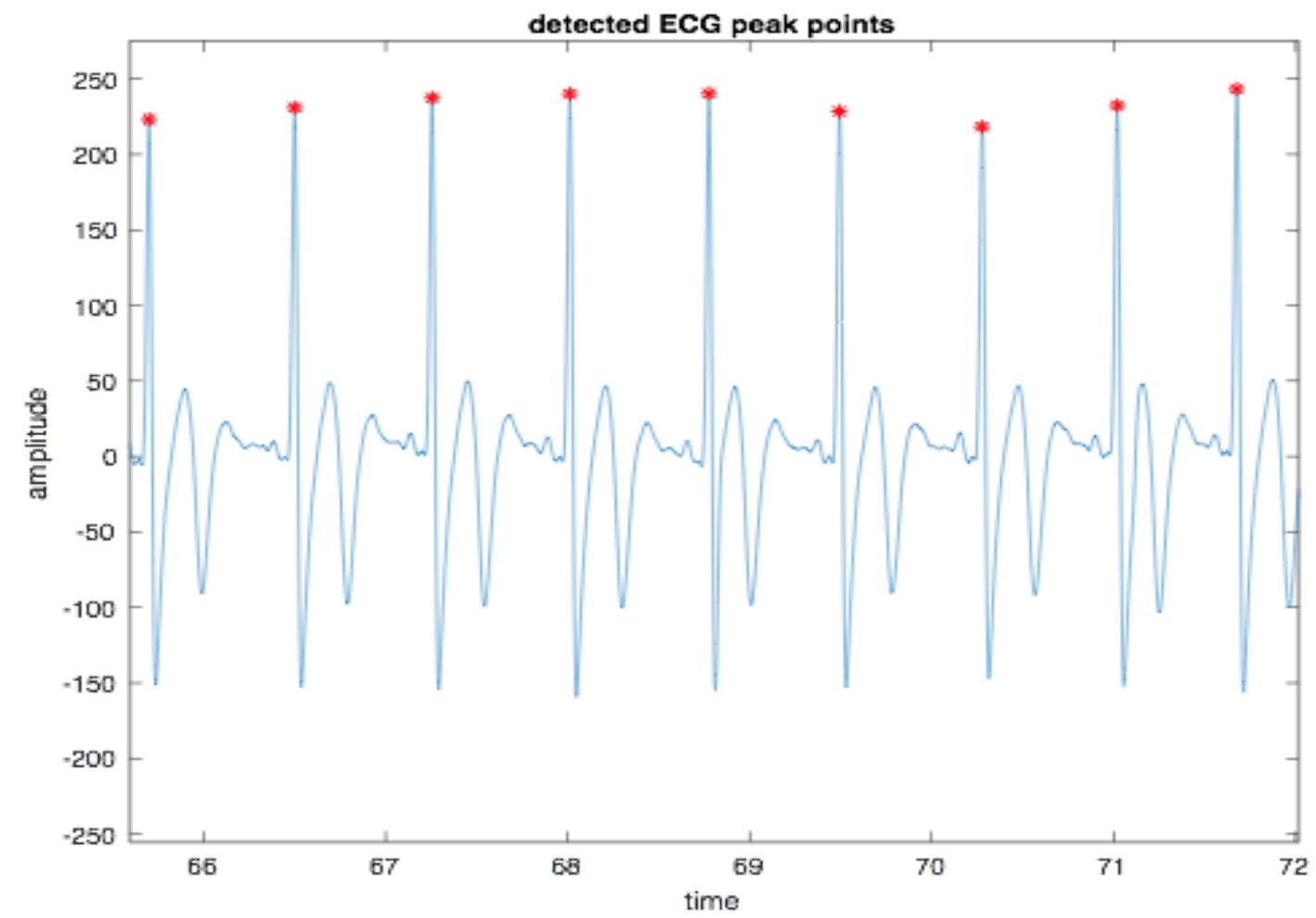

Figure 39: Detected $R-R$ peaks from the color stroop test (CST). Time in seconds 


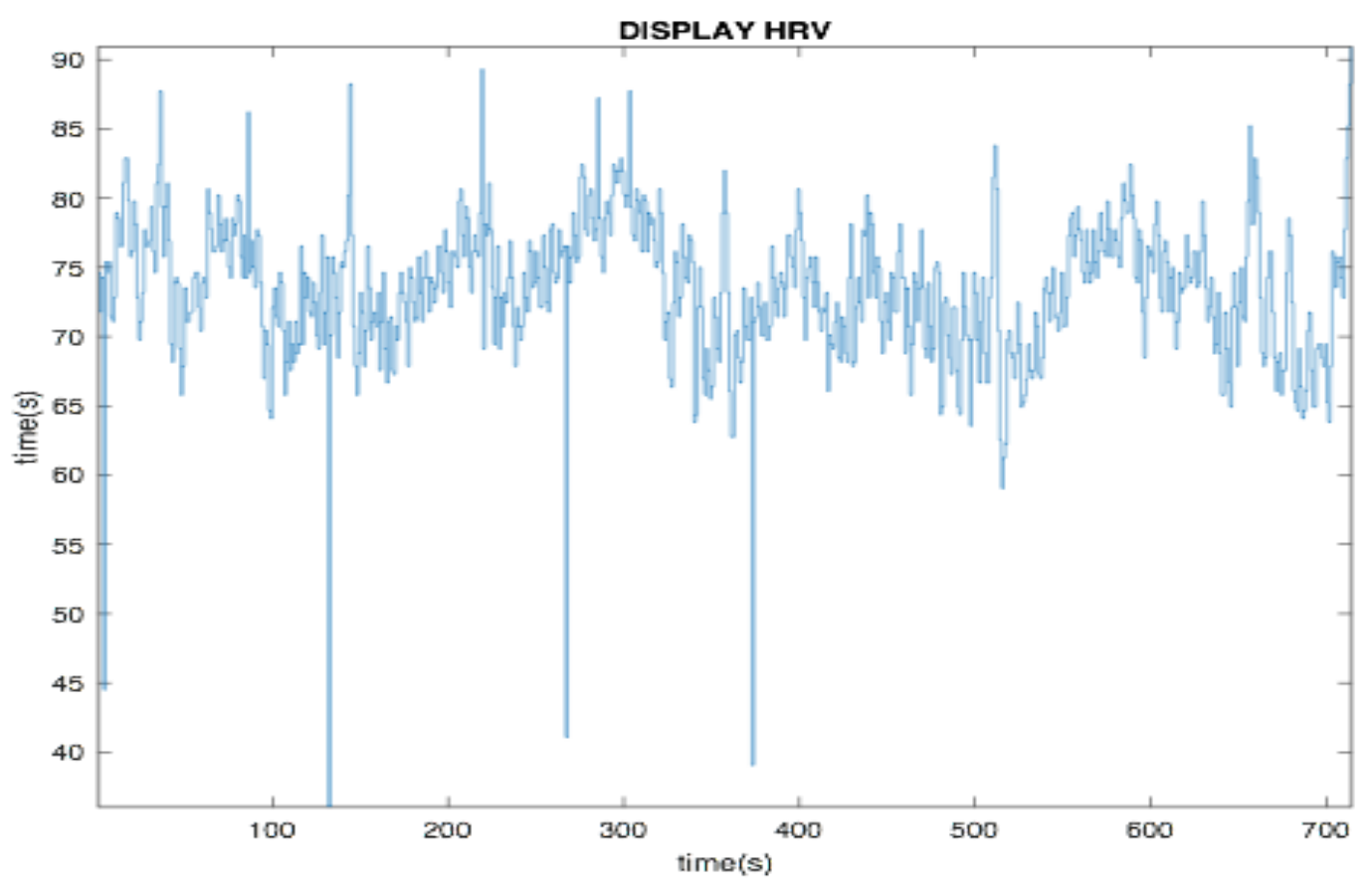

Figure 40: HRV signal which represents variation between Heart beats from CST.

Figure 39 and 40 represent the detected R-peaks and HRV signal obtained from the color stroop test, it's a cognitive game which measures the subject's level of attention, mental vitality and flexibility and it was only 5 minutes. Although user 2 had a slight increase with a mean hr of 76.0547, it does not indicate anxiety. The subjects $S D N N$ increased to $0.16082, R M S S D$ increased all the way to $0.21156,148 \mathrm{NN}$ intervals were greater than $50 \mathrm{msec}(N N 50)$, and pNN50 increased to 0.25641 , the subject seemed to adapt well to the game which indicates a good level of attention and mental flexibility during phases which may induce stress through negative valence (negative emotion towards roller coaster) and increased anxiety (due to the inability to pick the right colour). HRV signal varies slightly which is an indication of being awake. 


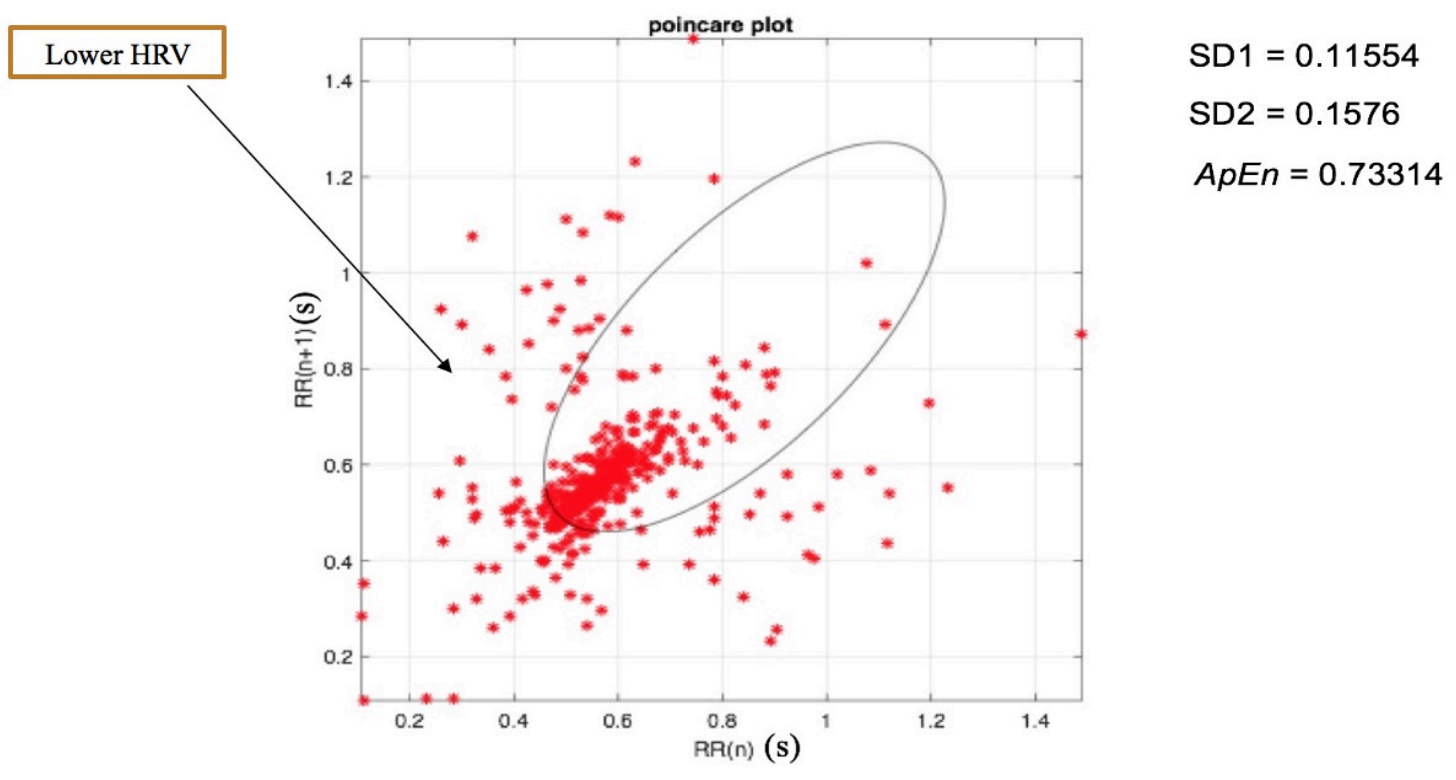

Figure 41: Poincare plot obtained from stroop task. $R R$ interval measured in seconds. The HRV further reduced from previous roller coaster phase.

Peaks points from the Poincare plot displayed in Figure 41 are positioned around the lower-mid corner which indicates normal HRV. Both SD1 and SD2 value increased to $0.11554,0.1576$ seconds respectively, ApEn increased again to 0.73314 which indicates a lower variation of HRV during the cognitive task in comparison to baseline and roller coaster phase. Mild boredom is often associated with low level of mental stimulation and increased fatigue, which might be the reason behind the low activity level and higher HR during the baseline phase, in comparison to mentally stimulating VR roller coaster phase and cognitive stroop task. 


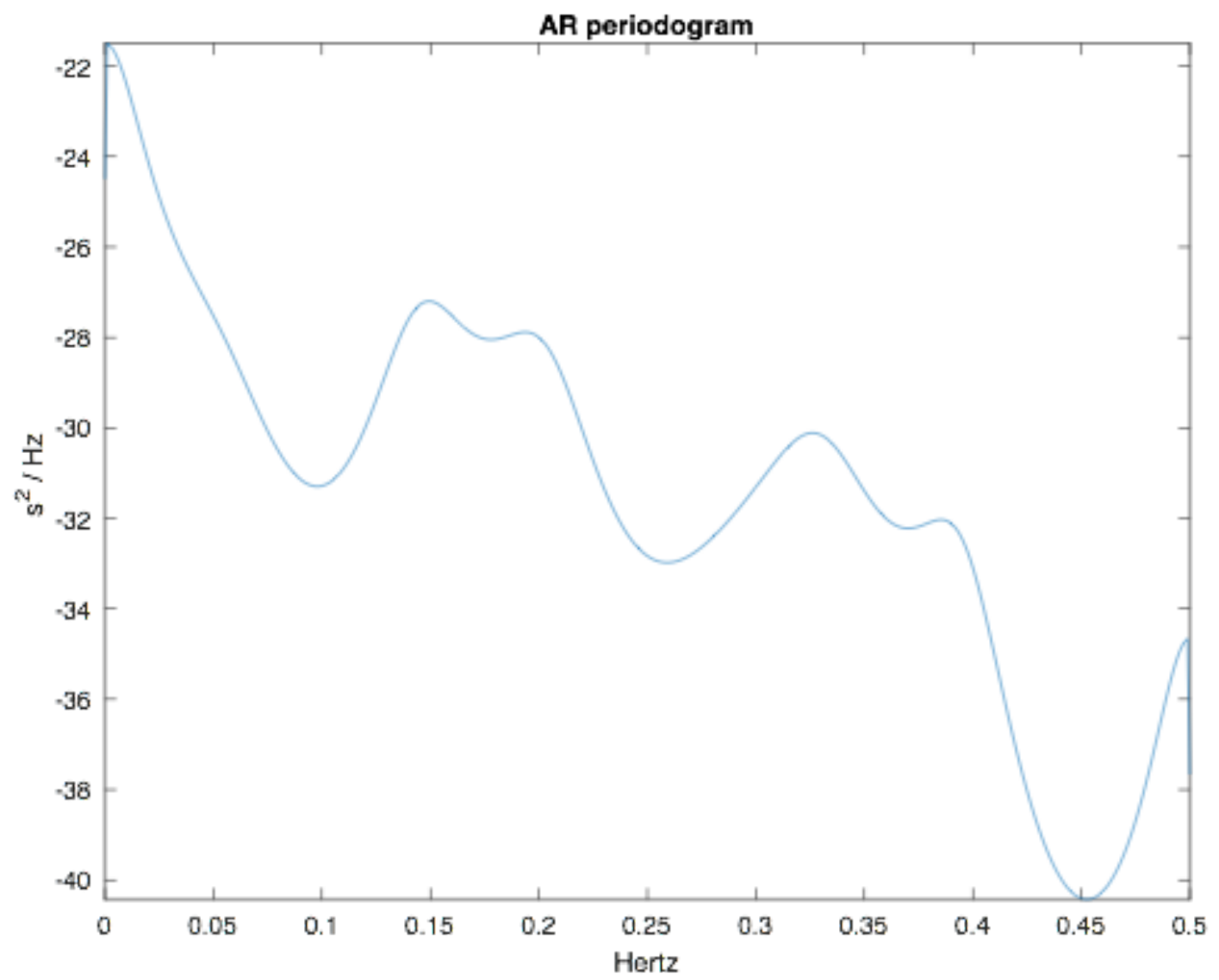

Figure 42: AR periodogram obtained from color stroop task

Figure 42 portrays the AR periodogram representing the subject's PSD during the color stroop task, this task is primarily used to assess attention and mental flexibility but may cause anxiety as well depending on the person's mental state. User 2 seemed to enjoy reaction games and was fairly relaxed during this process. The subject results indicate PNS activity dominance which was indicated through a decrease in $V L F$, it reduced during this phase, resulting in 171.8602 $\mathrm{ms}^{\wedge} 2, L F$ resulted in a value of $140.6917 \mathrm{~ms}^{\wedge} 2, H F$ was greater than $L F$ during this phase with a value of $235.7548, L F / H F$ reduced below 1 to 0.5968 , which is an indication of being more relaxed and the subject had a total power of $558.5869 \mathrm{~ms}^{\wedge} 2$. 


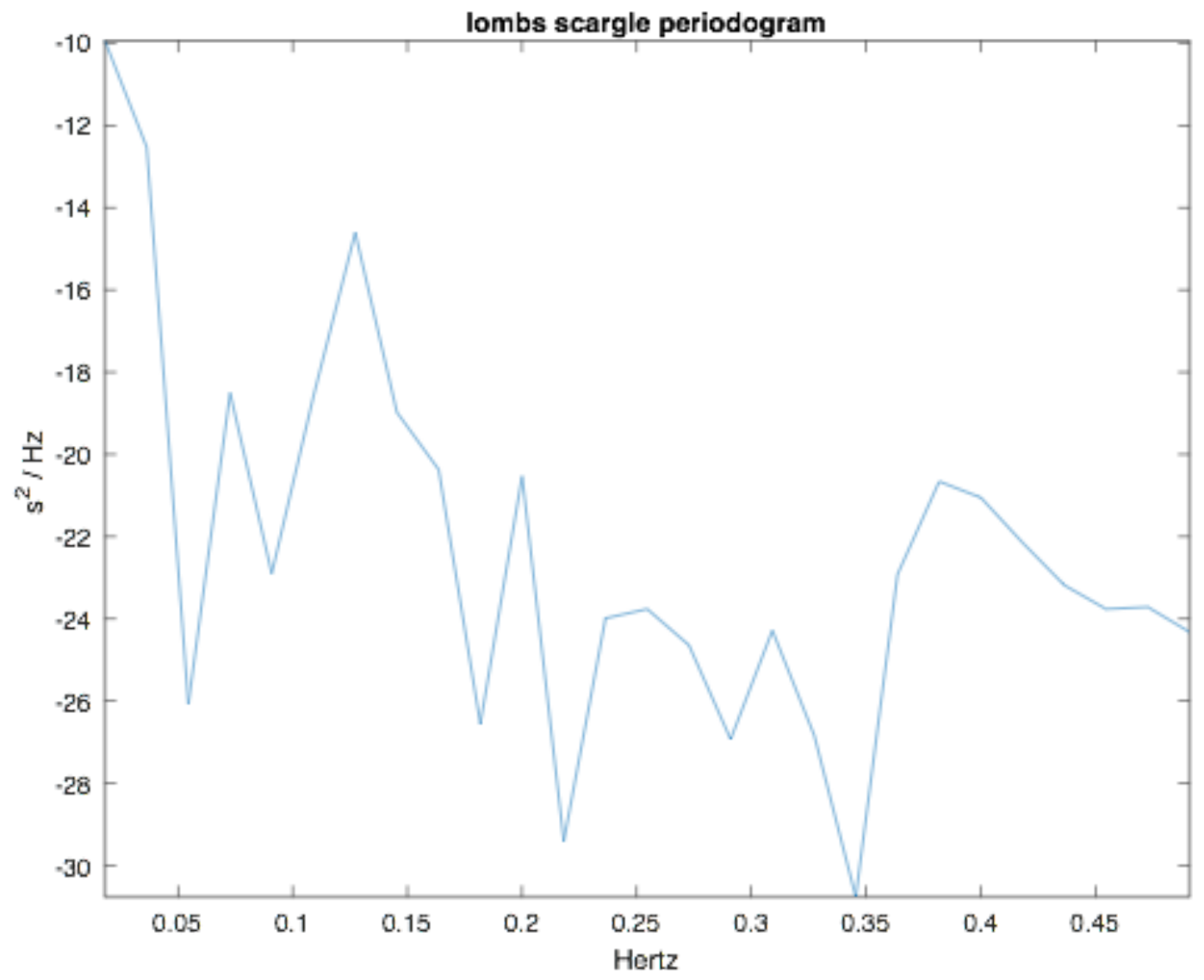

Figure 43: Lomb Scargle Periodogram portraying ANS activity during stroop task.

Figure 43 exhibits the PSD obtained through Lomb Scargle Periodogram during the color stroop task, the results do not indicate a large variation between the roller coaster phase and stroop task, although the subject's result indicate that he was more aroused during this phase which is indicated through a slight increase in $L F / H F$ ratio. The $V L F$ during this phase was $2.8998 \mathrm{e}+03$ $m s^{\wedge} 2$, the $L F$ value was $2.6878 \mathrm{e}+03 m s^{\wedge} 2$, the $H F$ decreased from roller coaster phase which is depicted through a value of $1.4916 \mathrm{e}+03 m s^{\wedge} 2, L F / H F$ slightly increased resulting in 1.8019 and the total noticed a minimal decreased as indicated through a value of $5.9301 \mathrm{e}+03 \mathrm{~ms}^{\wedge} 2$. 


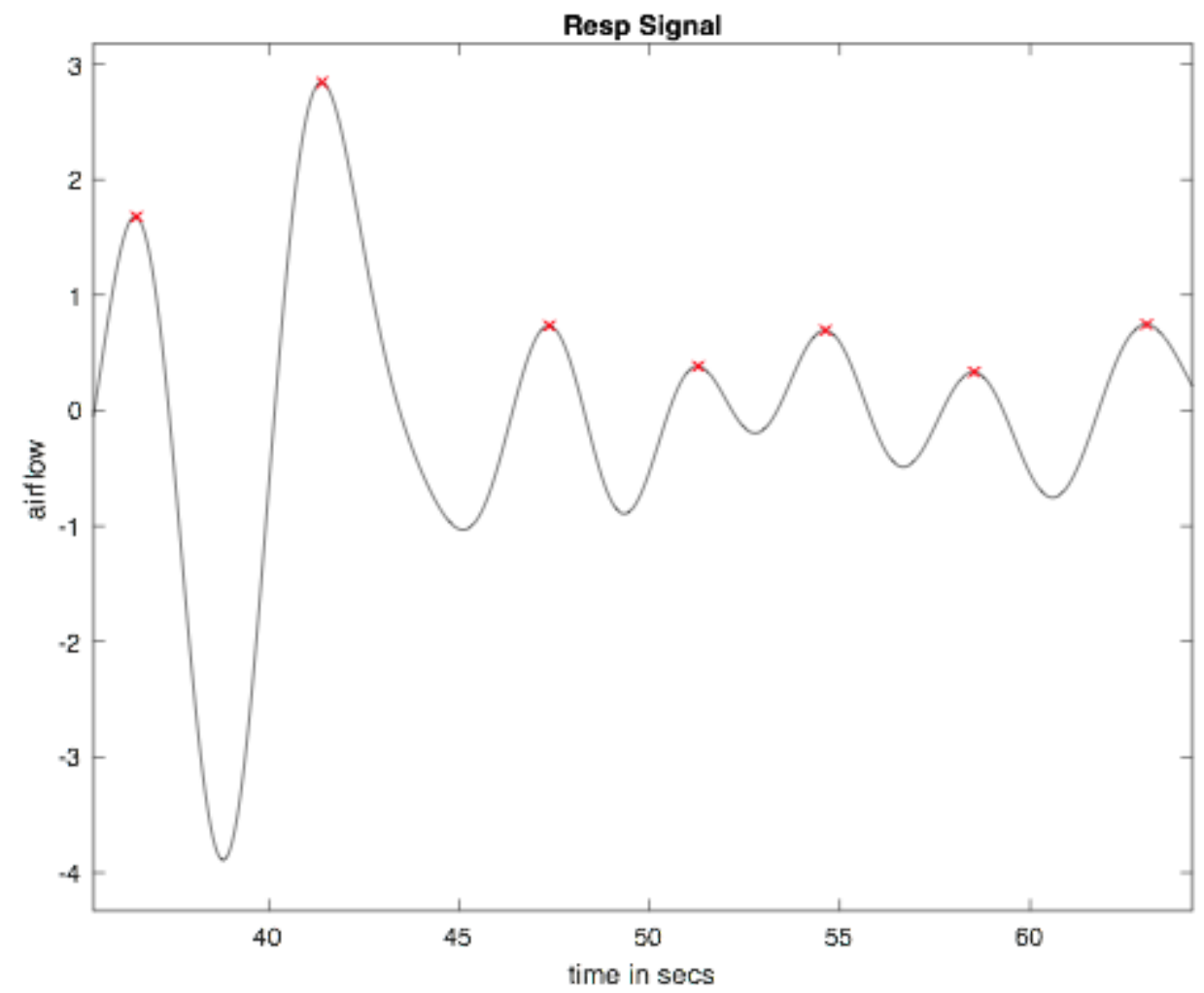

Figure 44: Respiration signal during color stroop task. BR- 15.7510

Figure 44 depicts the respiration signal during color stroop task. The game can cause impatience due to the inability to finish the game but a normal respiration rate of $15.7510 \mathrm{breaths} / \mathrm{min}$ indicates that the subject was calm and flexible (about not winning, just having fun playing the game) during the game. Greater than $30 \%$ of respiration signals are associated with error. 

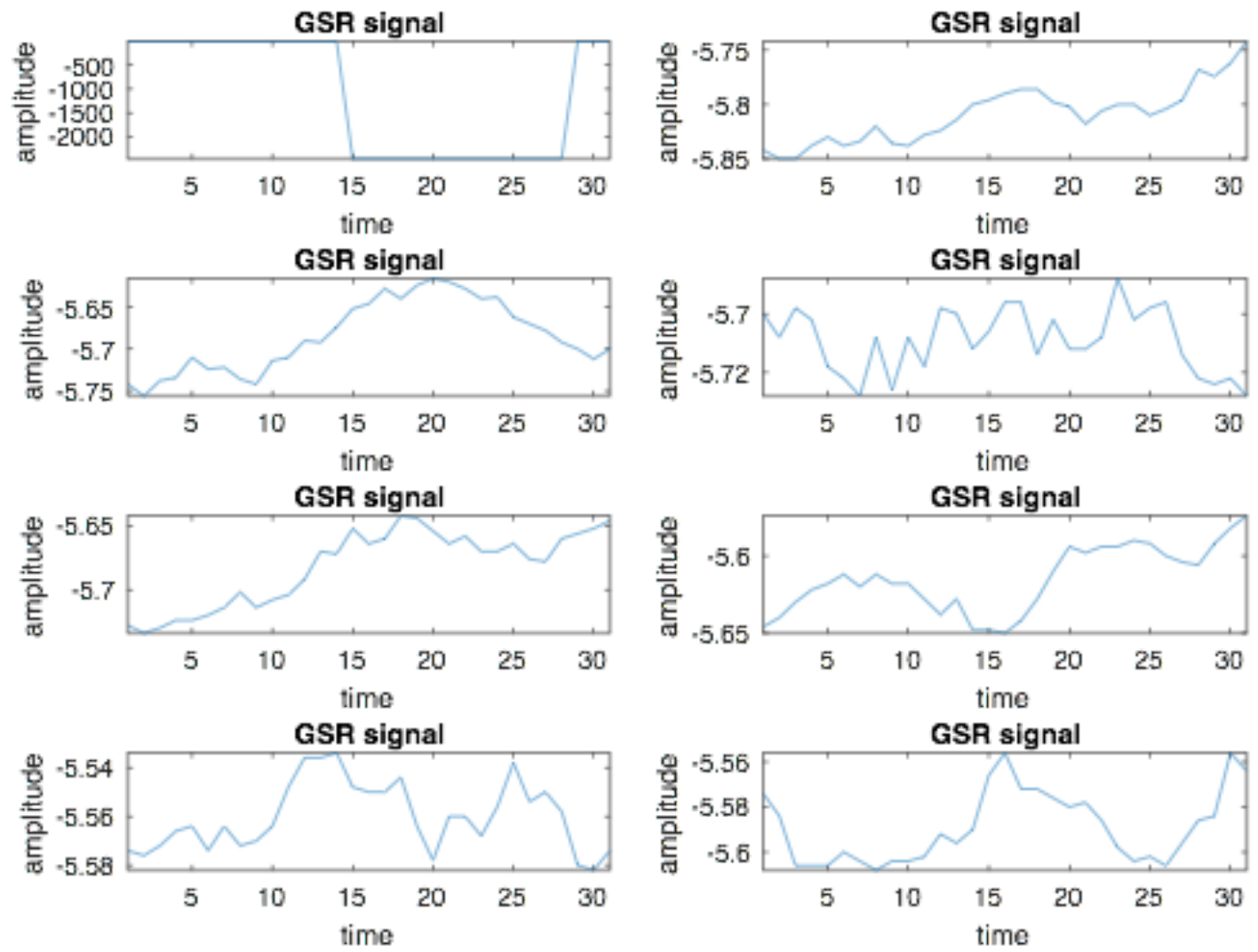

Figure 45: GSR obtained from Stroop Task. Mean 5.664. Std 0. $02332 \mu S / s e c$. Time in seconds

The GSR obtained from stroop task resulted in a mean GSR of $5.665 \mu S / s e c$ and GSR std of $0.02332 \mu \mathrm{S} / \mathrm{sec}$, respectively. The subplot using a $30 \mathrm{sec}$ window is displayed in Figure 45. Plot 3 indicates an instantaneous stimulus at 20 seconds which is the 80 seconds mark. Plot 4 has an instantaneous stimulus at 24 seconds which is the 114 seconds mark. Plot 8 has an instantaneous stimulus at 16 seconds which is the 226 seconds mark (3:46 min). Instantaneous stimulus is associated with stress.

\subsubsection{VR Fishing Game - Relaxation phase}




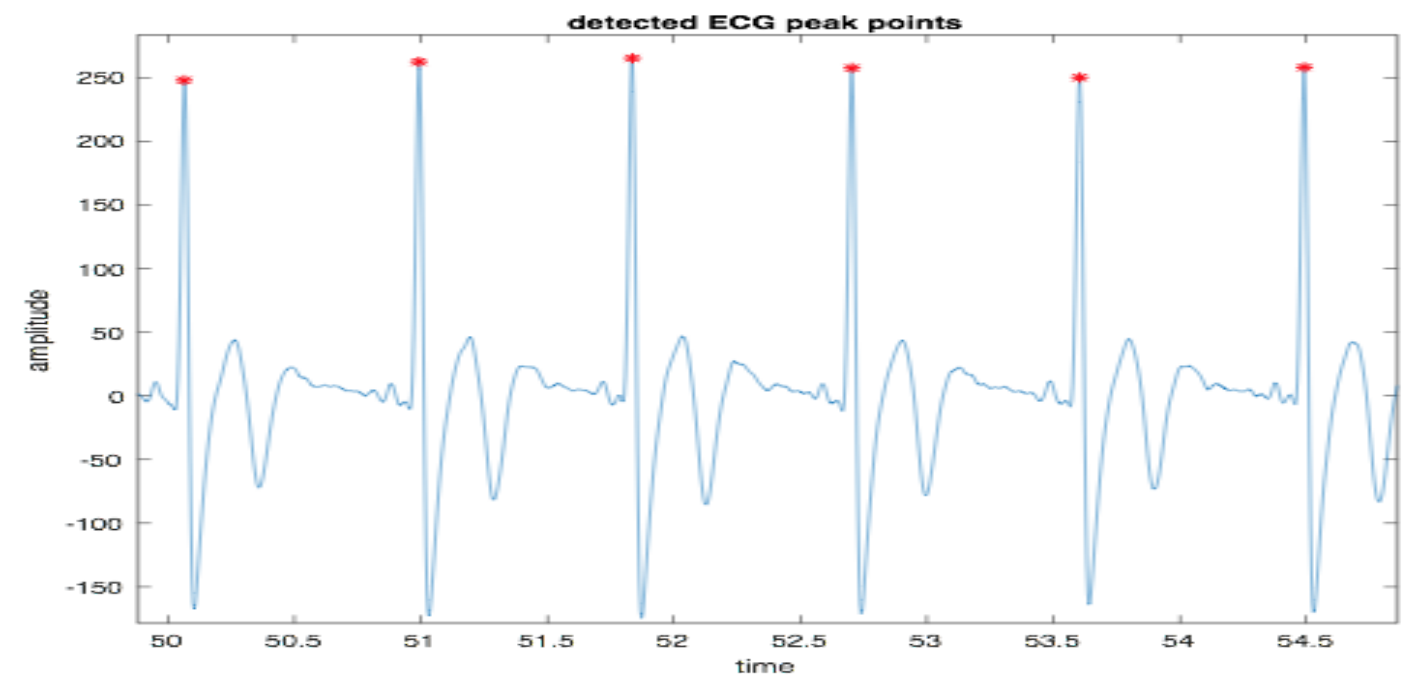

Figure 46: $R-R$ peaks obtained from VR fishing game

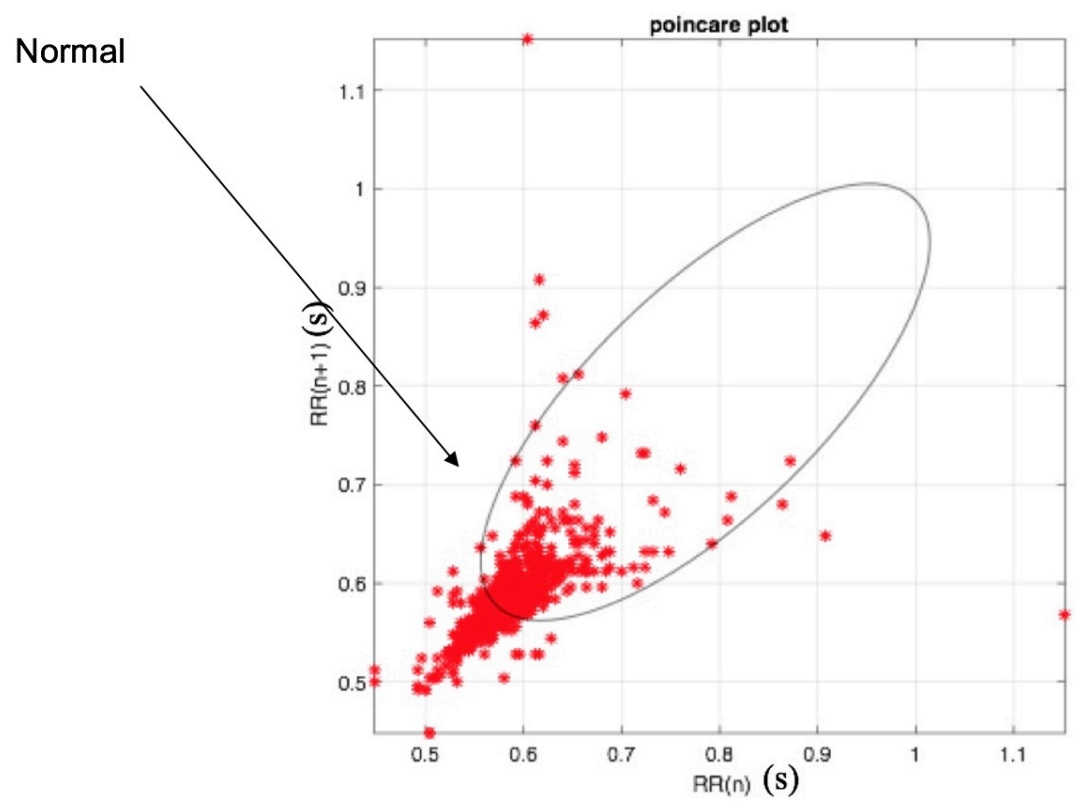

SD1 $=0.037823$
SD2 $=0.069271$
$A p E n=0.54602$

Figure 47: Poincare plot which is indicative of normal physiological function. $R R$ intervals are measured in seconds.

Figure 46 and 47 represent the $R-R$ peaks and Poincare plot obtained from VR fishing game, it was 5 minutes in duration and was developed with the idea of inducing relaxation within subjects. Subject had a slight decrease HR, which was a mean hr of 74.2265 , which is associated 
with slight increase in HRV, SDNN and RMSSD decreased to 0.084564 and 0.10201 respectively. $87 \mathrm{NN}$ differed by $50 \mathrm{msec}(N N 50), p N N 50$ slightly decreased to 0.2491 . PNN50 shares a good correlation with HRV, it is higher than baseline and roller coaster phase which indicates that the fishing game is more relaxing than those phases but slight decrease from stroop task may indicate that the subject was more enthusiastic about the cognitive game and less bored.

The $S D 1$ and $S D 2$ values decreased to $0.037823,0.069271$ seconds and $A p E n$ decreased to 0.54602 .

Non-linear parameter $(S D 1, S D 2, A p E n)$ varied, increased with increase in $p N N 50$ and decreased during decrease in $p N N 50$, which may indicate a positive correlation with HRV, $p N N 50$. The decrease in $A p E n$ reinforce the assumption that the subject was a little bit more relaxed following the VR fishing game.

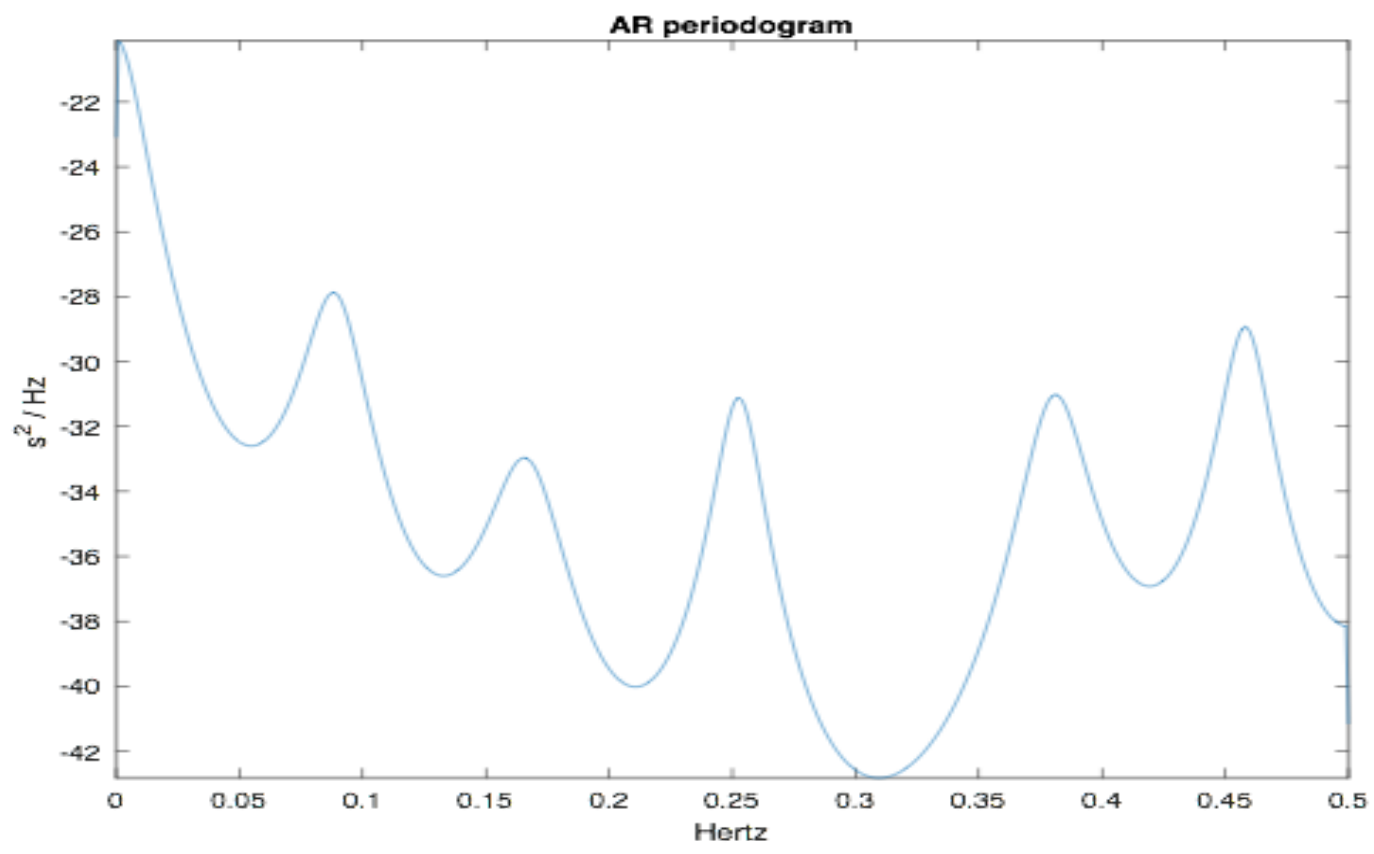

Figure 48: AR periodogram obtained during VR fishing game.

Figure 48 depicts AR periodogram of the subject due to VR fishing game, PSD indicates whether it was effective in reducing stress through the depiction of SNS and PNS activity. During this phase the subject had a $V L F$ of $141.1033 m s^{\wedge} 2, L F$ of $73.2178 m s^{\wedge} 2, H F$ of $66.1596 m s^{\wedge} 2$, 
$L F / H F$ ratio of 1.1067 and a total power of $321.0365 \mathrm{~ms}^{\wedge} 2$. The results from the $L F / H F$ indicates that his ANS activity was fairly balanced and the subject was awake after the whole process.

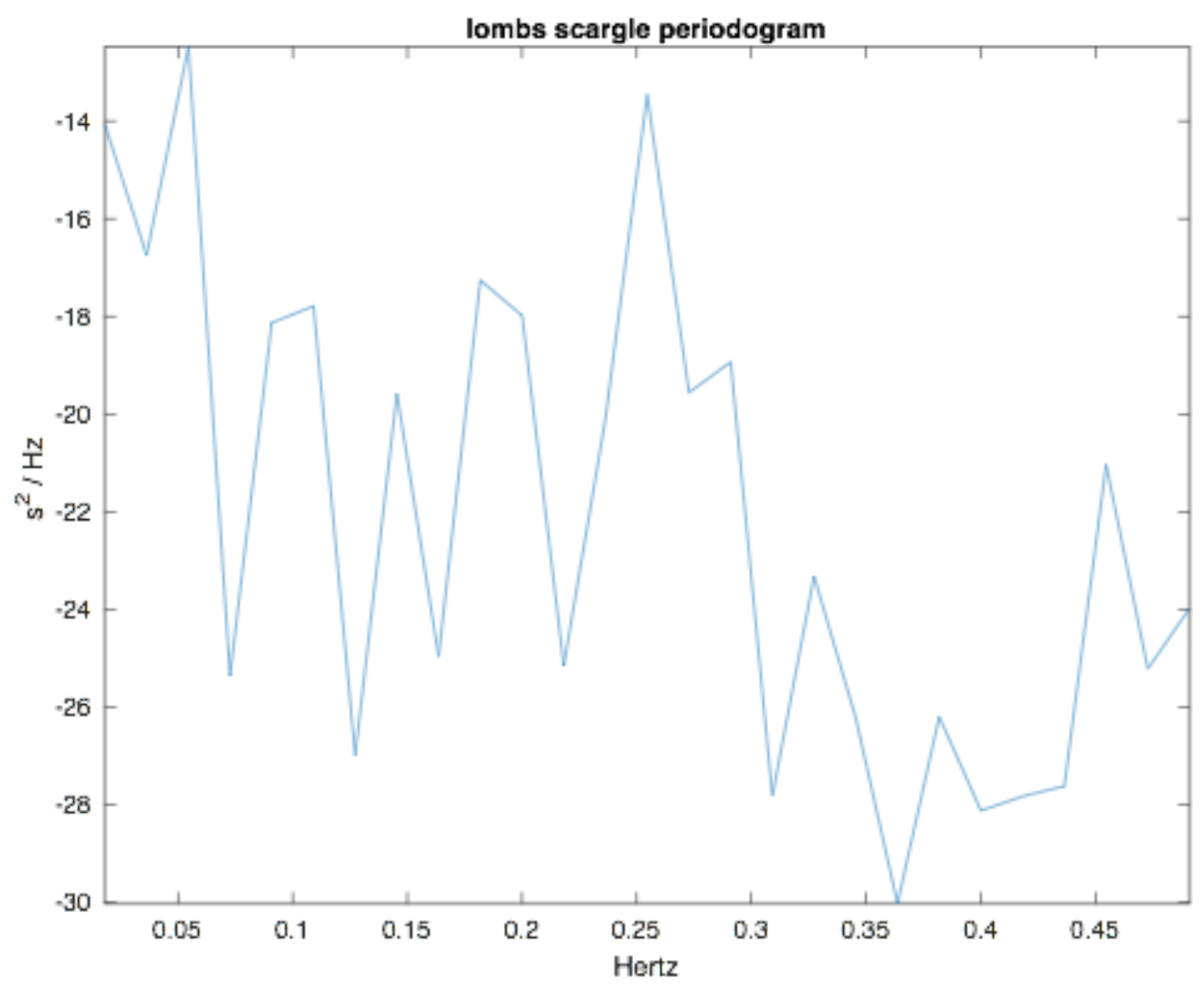

Figure 49: Lomb Scargle periodogram due to VR fishing game.

Figure 49 indicates PSD of the VR fishing obtained through Lomb Scargle periodogram method. The results did not vary too differently from the stroop task, although $L F / H F$ decreased which was coherent with the purpose of this task. The subjects had a $V L F$ of $2.1232 \mathrm{e}+03 m s^{\wedge} 2, L F$ of $2.3397 \mathrm{e}+03 \mathrm{~ms}^{\wedge} 2, H F$ value increased during this phase resulting in $2.6527 \mathrm{e}+03 \mathrm{~ms}^{\wedge} 2, L F / H F$ ratio was below 1 with a value of 0.8820 and total power was $5.7485 \mathrm{e}+03 \mathrm{~ms}^{\wedge} 2$. VR results indicate that the game induced relaxation within the subject, who was emotionally and mentally stimulated by the VR roller coaster simulation and cognitive colour stroop task. 


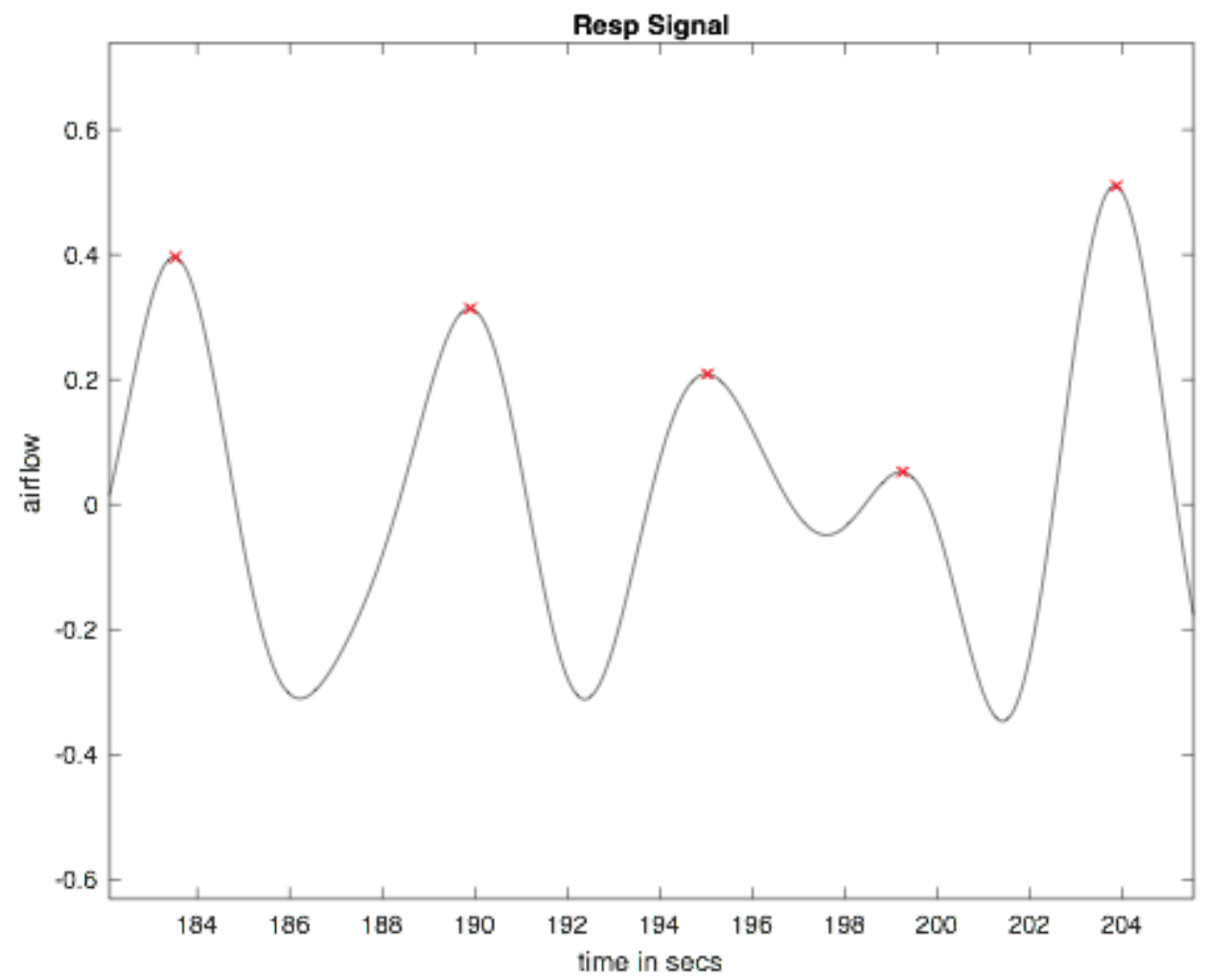

Figure 50: Respiration signal during fishing game

The subject's respiration rate decreased to 12.9263 breaths/min during the VR fishing game simulation as described in Figure 50. Further decrease in respiration rate indicates that the fishing game does calm down the subject (he was quite normal during the cognitive stroop task). The VR headset can interfere with breaths detected, the electrodes were not directly attached to the chest, which may lead to abnormally high/low breathing rates as well. 

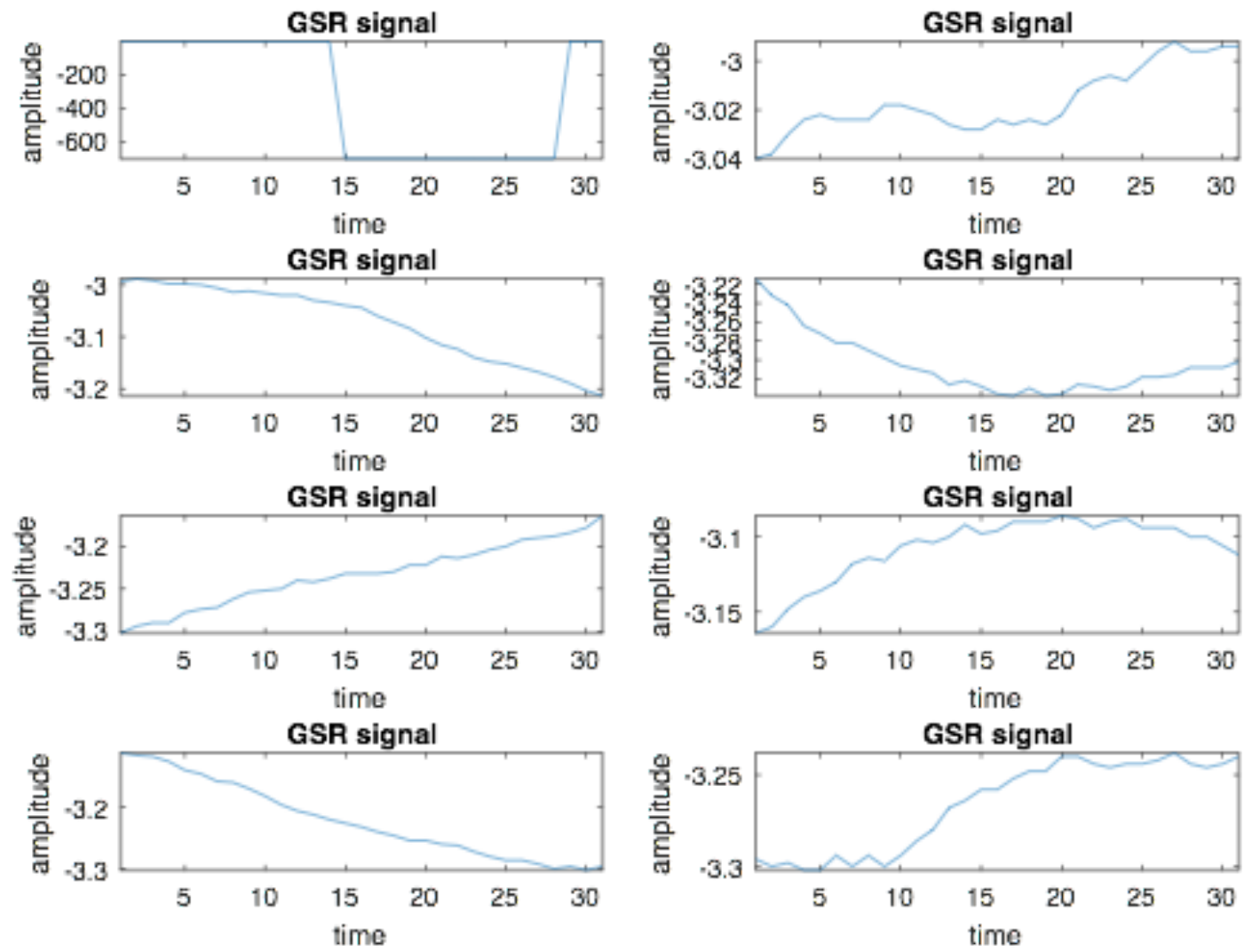

Figure 51: GSR signals obtained from VR fishing game. Time in Seconds

Figure 51 displays the GSR signals obtained from VR fishing game, the signals are split into 30 seconds windows in ascending order from left to right, top to bottom. There was no stimulus (indicates stress stimulated during a certain period) detected as indicated through the graphs, which is coherent with the purpose of trying the calm the subject down after inducing stress through roller coaster and stroop task. The subject has a mean GSR value of $3.257 \mu \mathrm{S} / \mathrm{sec}$ and a GSR std value of $0.0379 \mu \mathrm{S} / \mathrm{sec}$. 


\begin{tabular}{|c|c|c|c|c|c|}
\hline User 2 & T1 & $\mathrm{T} 2$ & T3 & T4 & $p$-value \\
\hline Mean HR & 84.7597 & 73.4106 & 76.0547 & 74.2265 & 8.50E-05 \\
\hline SDNN & 0.053441 & 0.07269 & 0.16082 & 0.084564 & 0.029 \\
\hline RMSSD & 0.031241 & 0.083187 & 0.21156 & 0.10201 & 0.0667 \\
\hline NN50 & 21 & 124 & 148 & 87 & 0.0415 \\
\hline PNN50 & 0.20243 & 0.14045 & 0.25641 & 0.24691 & 0.0041 \\
\hline SD1 & 0.022112 & 0.058863 & 0.14979 & 0.072218 & 0.0667 \\
\hline SD2 & 0.072271 & 0.084277 & 0.17115 & 0.095325 & 0.0178 \\
\hline ApEN & 0.30596 & 0.69844 & 0.87521 & 0.7576 & 0.0128 \\
\hline $\operatorname{VLF}\left(m s^{\wedge} 2\right)(A R)$ & 12.4056 & $1.71 \mathrm{E}+02$ & $1.72 \mathrm{E}+02$ & $1.41 \mathrm{E}+02$ & 0.0467 \\
\hline$L F\left(m s^{\wedge} 2\right)(A R)$ & 3.9326 & $7.71 E+01$ & 140.6917 & $7.32 \mathrm{E}+01$ & 0.0777 \\
\hline$H F\left(m s^{\wedge} 2\right)(A R)$ & 12.433 & $4.89 E+01$ & $2.36 \mathrm{E}+02$ & $6.62 E+01$ & 0.1646 \\
\hline LF/HF (AR) & 0.3163 & 1.5787 & 0.5968 & 1.1067 & 0.0485 \\
\hline TP (AR) & 30.5408 & $3.25 E+02$ & $5.59 \mathrm{E}+02$ & $3.21 E+02$ & 0.0648 \\
\hline $\operatorname{VLF}\left(\mathrm{ms}^{\wedge} 2\right)(\mathrm{Lor}$ & 400.2059 & $2.08 \mathrm{E}+03$ & $2.90 \mathrm{E}+03$ & $2.12 \mathrm{E}+03$ & 0.0378 \\
\hline $\operatorname{LF}\left(\mathrm{ms}^{\wedge} 2\right)$ (Lomk & 846.711 & $3.03 E+03$ & $2.69 \mathrm{E}+03$ & $2.34 \mathrm{E}+03$ & 0.019 \\
\hline $\mathrm{HF}\left(\mathrm{ms}^{\wedge} 2\right)(\mathrm{Lom}$ & 432.6174 & $2.66 \mathrm{E}+03$ & $1.49 \mathrm{E}+03$ & $2.65 E+03$ & 0.043 \\
\hline LF/HF (Lomb) & 1.9572 & 1.1411 & 1.8019 & 0.882 & 0.0112 \\
\hline TP (Lomb) & $1.52 \mathrm{E}+03$ & $6.14 E+03$ & $5.93 E+03$ & $5.75 E+03$ & 0.0222 \\
\hline GSR_mean & 3.175 & 6.2054 & 5.665 & 3.257 & 0.0103 \\
\hline GSR_std & 0.0379 & 0.07399 & 0.02332 & 0.0379 & 0.0279 \\
\hline RESP(breath/m & 10.223 & 24.832 & 24.9577 & 24.3454 & 0.0101 \\
\hline
\end{tabular}

Table 2: User 2 results obtained from baseline (T1) - VR fishing game (T4) phase. The p-value indicates if the corresponding results are significant (marked in red).

Table 2 displays the results from each phase of the experiment, starting with the initial baseline phase and ending with VR fishing game. Statistical analysis was computed through MATLAB using the t-test to test whether the data was significant (indicated through red). P-value $>0.05$ reject the null hypothesis that the data are statistically significant, belong to the same group and there is no significant difference between the data. 


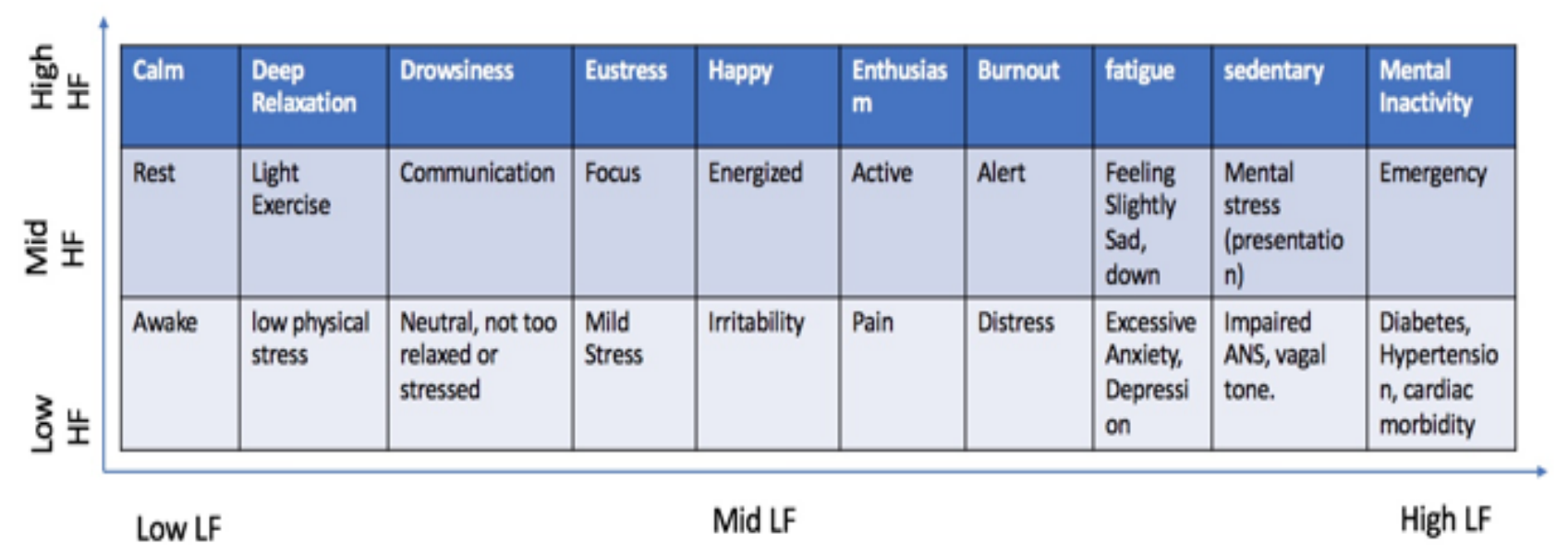

Figure 52: Physical, physiological and behavioural symptoms associated with $L F, H F$ variations.

Figure 52 presents a chart which identifies the mental, physical, physiological and behavioural symptoms associated with $L F$ and $H F$ variations. Ideally $L F$ and $H F$ was utilized to represent the sympathovagal balance, but numerous research studies observed the $L F$ and $H F$ variations associated with stress, cardiac morbidities and other symptoms which are listed on the table above.

\subsection{Discussion}

The purpose of the experiment was to induce stress through a VR roller coaster simulation and cognitive stroop task. A total of 166 signals were analyzed through ECG, GSR, RESP sensors and MATLAB software. The ECG signals were filtered with a Notch filter and smoothed using wavelet transform to pre-process the ECG signal and obtain time domain HRV parameter. AR and Lomb Scargle periodogram models were used to transform the time domain parameters into frequency domain power spectral density (PSD). HRV parameters from both time and frequency domain were analyzed in order to assess the level of stress/relaxation associated with each phase. GSR signal was low pass filtered and segmented into 830 seconds window, it can only be utilized to assess stress through peaks associated with an instantaneous stimulus due to variations associated with skin conductance, PNS does not innervate eccrine sweat gland, therefore it 
cannot be analyzed by GSR. Respiration signal was very noisy and was filtered with a point specific band-pass filter, stress and strong emotions can cause rapid breathing or shortness of breath due to constricted airways between the nose and lungs.

Results were obtained from both time and frequency domain, (Rosenberg, et al., 2017) had revealed that frequency domain parameters were more efficient for analyzing HRV, unlike time domain parameters, frequency domain parameter can measure the changes associated with SNS and PNS activity more effectively [2]. The results obtained for 14 subjects further accentuate the previous hypothesis that frequency domain parameters were superior to time domain parameter with regards to stress analysis, frequency domain parameters resulted in larger variations when transitioning from a neutral phase to a stressful phase. The results obtained from subject 2 indicate that $p N N 50, L F$ and $L F / H F$ presented a noticeable variation during roller coaster phase. PNN50 decreased from 0.202 to $0.14(\mathrm{p}=0.0041), L F$ frequency increased from 3.9326 to $77.153 \mathrm{~ms}^{\wedge} 2(\mathrm{p}=0.077), L F / H F$ ratio increased from 0.3163 to $1.5787(\mathrm{p}=0.0485)$ during VR roller coaster simulation after the baseline (neutral) phase. VR roller coaster phase aroused the subjects into a state of wakefulness through stressors (stress stimulus) associated with the VR roller coaster environment. Low $L F$ and $L F / H F$ ratio was initially associated with a state of drowsiness due to high $H F$ and low $L F$ (during the baseline phase). PNN50 which is correlated with $H F$ and HRV decreased due to an increase in $L F$ and $L F / H F$ ratio, as a result of increased arousal/wakefulness due to stressors. $\mathrm{P}<0.05$ which indicates that the resulting values are significant enough to be considered from the same feature, $p>0.05$ would indicate that the resulting value may be too large to be considered right often as a result of excessive noise, motion artifacts . 

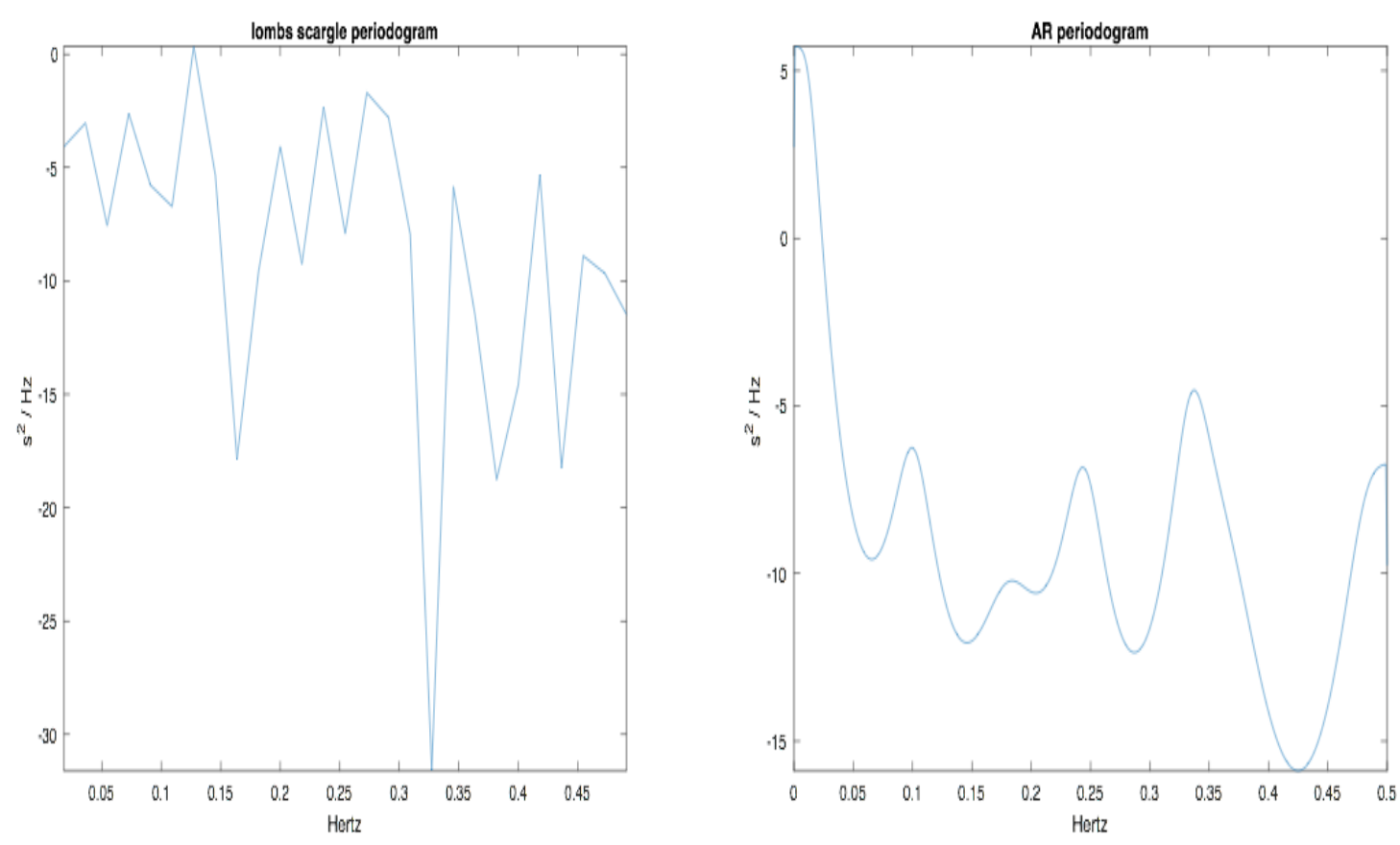

Figure 53: AR and Lomb Scargle periodogram PSD obtained during color troop task for User 10 .

User 10 (Figure 53) had a neutral $V L F$ of $139\left(m s^{\wedge} 2\right)(\mathrm{p}=0.1022), L F$ of $103\left(m s^{\wedge} 2\right)(\mathrm{p}=$ $0.0457)$ and $L F / H F$ ratio of $0.5426(\mathrm{p}=0.0465)$ which was obtained using AR method. The VLF and $L F$ are fairly similar, $L F$ primarily represents SNS activity while $V L F$ have been associated with adverse outcomes. Higher values within both frequencies is often associated with increased stress and SNS activity is more dominant within those frequencies. Both $L F$ and VLF are indicators of mortality, they are inversely proportional to HRV, increase in those frequencies has been associated with impairment of the ANS. A low $L F / H F$ indicates that initially $H F / \mathrm{PNS}$ activity was more dominant which is associated with relaxation. Lomb Scargle often results in higher values in comparison to AR method, initially user 10 (Figure 43) had a VLF of 2930 $\left(m s^{\wedge} 2\right)(\mathrm{p}=0.1562), L F$ of $1.10 \mathrm{E}+04\left(m s^{\wedge} 2\right)(\mathrm{p}=0.1991)$ and $L F / H F$ of $0.5948(\mathrm{p}=0.1697)$ using the lomb scargle periodogram method. Although Lomb Scargle PSD values were larger, both methods present a low $L F / H F$ around 0.54-0.59 which further strengthens the initial analysis of a relaxed user 10 during baseline phase. During the cognitive stroop task user 10 noticed an increase in $V L F$ up to $444\left(m s^{\wedge} 2\right)(\mathrm{p}=0.0 .1022), L F$ also increased to $133\left(\mathrm{~ms}^{\wedge} 2\right)(\mathrm{p}=$ $0.0457)$ and $L F / H F$ ratio also increased to $0.925(\mathrm{p}=0.0465)$ which was depicted through the AR model. Lomb scargle periodogram indicates that during the color stroop task the VLF of user 
10 increased to $1.93 \mathrm{E}+04\left(m s^{\wedge} 2\right)(\mathrm{p}=0.1562), L F$ increased to $5.62 \mathrm{E}+04\left(m s^{\wedge} 2\right)(\mathrm{p}=0.1991)$ and $L F / H F$ ratio increased to 0.8317 ( $\mathrm{p}=0.1697)$. Both AR and Lomb method resulted in increased $V L F, L F, L F / H F$ due to the cognitive color stroop task, although lombs periodogram resulted in values which were questionably high, noise and ectopic beats can result in inflated values and further interpolation may be required to retrieve a more accurate solution. Stroop task is an effective method to assess executive function, which was revealed by (Morgan \& Lilienfeld, 2000), it is associated with planning, directing, maintaining attention, organization, abstract reasoning, problem-solving, self-regulation, and motor control [89]. Stroop task requires the subject to be mentally flexible and attentive, the increase in $V L F, L F$ and $L F / H F$ values indicates that acute stress is associated with wakefulness and increased attention. The Prefrontal cortex (PFC), basal ganglia, anterior cingulate cortex (ACC), and posterior parietal cortex were the primary brain regions activated during cognitive flexibility tasks such as color stroop task (which is impossible to win and displays wrong colours with a respective word used to describe the colour) [89]. The values do not indicate increased anxiety due to the lack of mental flexibility.
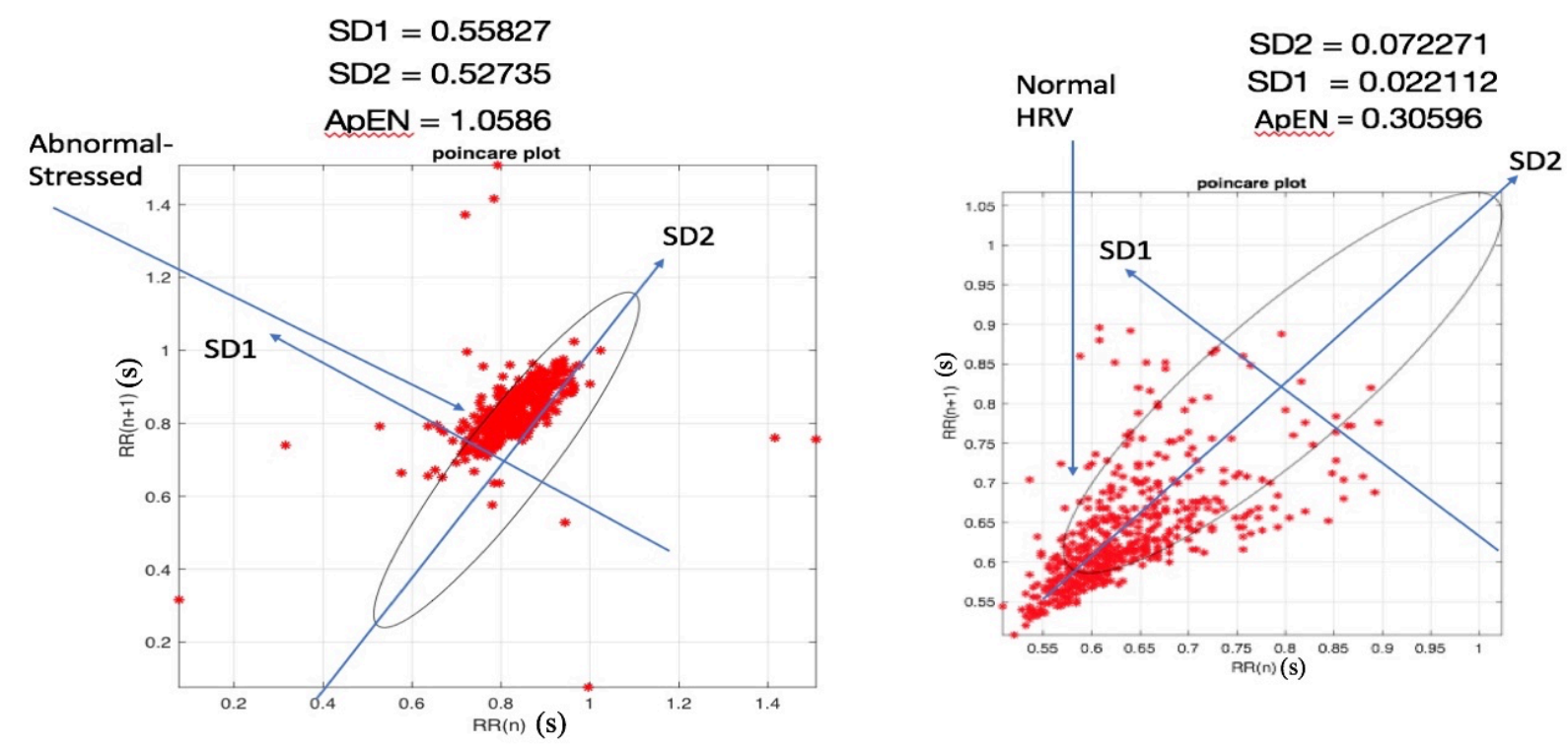

Figure 54: Visual Representation describing an abnormal and normal HRV on a 89oincare plot 
Similarly, ApEn (approximate entropy) obtained from 90oincare plot described a similar pattern as HF, TP (obtained from AR method), it represents the ratio between non-linear SD1 and SD2 used to assess HRV. For User 2 the ApEn at baseline was $0.30596(\mathrm{p}=0.0128)$, which increased to $0.69844(\mathrm{p}=0.0128)$ during roller coaster phase, further increased during cognitive stroop task to $0.87521(\mathrm{p}=0.0128)$, and reduced as desired during the relaxing VR fishing game down to $0.7576(\mathrm{p}=0.0128)$. User 10 followed a similar pattern, baseline phase resulted in ApEn of $0.48134(\mathrm{p}=0.0016)$, increased to $0.57412(\mathrm{p}=0.0016)$ during the VR roller coaster phase, further increased to $0.73314(\mathrm{p}=0.0016)$ during the stroop task and reduced down to $0.54602(\mathrm{p}$ $=0.0016$ ) during the VR fishing game. The results indicate variations associated with increased values during stress phases and decreased values during phases which were meant to be relaxing. (Li et al., 2014) also noticed that ApEn contained information which was associated with ANS disorders, the information they obtained was effective for distinguishing between healthy and diabetic patients [90]. ApEn measures the rate of unpredictability in $R$ - $R$ time series, it is also used to measure autonomic balance just like $L F / H F$ ratio, increasing during stressful events, morbidity and decreasing accordingly during relaxing phases such as VR fishing game [81]. The results also stipulate that VR fishing can reduce stress levels and increase resistance to stress.

Stress occurs as a result of perceived danger, which is associated with a person's perception, VR roller coaster simulation indicates through various results that the simulation increases $L F$, $L F / H F$ ratio and HR (user 10) which can be logically interpreted as increase in stress levels, but there were contradictions in the result. Lomb's periodogram indicated a decrease in $L F / H F$ and an increase $H F$ during the roller coaster phase but the subjects $L F$ also increased to indicate increased stress within the subject. Stress increased for both subjects, but $L F / H F$ ratio and $H F$ results indicate that subject 2 was also fairly relaxed as well. (Du, J et al., 2018) and psychologists have revealed that activity such as a roller coaster rides is perceived differently by different subjects, one subject may find it exhilarating whereas another might find it terrifying. Stress is stimulated within both subjects, but one subject is suffering from good stress, euphoria also known as eustress while the other is subjected to distress, which is bad and can induce negative emotions [91]. Eustress is good, helps a person focus, enjoy certain activities such as social events, roller coaster ride but a difference in perception can also trigger distress, some 
people perceive social activities as draining and roller coaster rides induce fear within some people. The results from this experiment further reinforce that theory, although stress is induced within all subjects (increased $L F$ ), some find the roller simulation to be fun, relaxing (decreased $H F, L F / H F$ ratio) while others found it to be terrifying, some found the stroop task to be fun and challenging, while it made other subjects anxious (increased $L F / H F$, increased $H R$, reduced HRV). (Du, J et al., 2018) has revealed that negative perception of stress can be used to predict anxiety symptoms, angry emotions which can lead to misbehaviour and substance abuse [91]. Stress can also be used to antedate depression, shame, excessive negative thoughts [91]. 


\begin{tabular}{|c|c|}
\hline $\begin{array}{l}\text { Physiological function before video game } \\
\text { (Average of all the users) }\end{array}$ & $\begin{array}{l}\text { Physiological function after video game } \\
\text { (Average of all the users) }\end{array}$ \\
\hline Heart Rate $($ beats $/ \mathrm{min})=103.52$ & Heart Rate (beats $/ \mathrm{min})=92.95$ \\
\hline$A p E n=0.695$ & $A p E n=0.562$ \\
\hline$L F\left(m s^{\wedge} 2\right)=3.55 E+05$ (Lomb) & $L F\left(m s^{\wedge} 2\right)=4.96 \mathrm{E}+03$ (Lomb) \\
\hline$H F\left(m s^{\wedge} 2\right)=2.2 E+05($ Lomb $)$ & $H F\left(m s^{\wedge} 2\right)=7.1 \mathrm{E}+03$ (Lomb) \\
\hline$L F / H F=1.2$ (Lomb) & $L F / H F=0.93$ (Lomb) \\
\hline GSR_mean $(\mu S / s e c)=5.665$ & $G S R \_$mean $(\mu S / \mathrm{sec})=3.257$ \\
\hline$R E S P($ respiration rate $/ \mathrm{min})=24.95$ & $R E S P($ respiration rate $/ \mathrm{min})=24.34$ \\
\hline Why is Video game great for Stress & Why are other methods less effective \\
\hline Enter a new world and escape reality & Is not a good positive distraction \\
\hline $\begin{array}{l}\text { Simple action which takes your mind off } \\
\text { of problems }\end{array}$ & Strenuous, painful, hard \\
\hline Brain stimulation which relaxes the brain & Boring and makes you feel worse \\
\hline $\begin{array}{l}\text { Effort leads to rewards. Logic and } \\
\text { reaction leads to good/results. }\end{array}$ & $\begin{array}{l}\text { Can make you think more about your } \\
\text { problems }\end{array}$ \\
\hline Relieves anxiety effortlessly & Requires a lot of effort \\
\hline
\end{tabular}

Figure 55: Impact of Video Games on physiological function and stress levels

VR fishing game resulted in decreased $L F / H F$ ratio, decreased HR, decreased $p N N 50$, decreased $A p E n$, decreased breathing rate, normal GSR which is associated with a more calm and relaxed state. Some subjects however noticed an increase in $L F / H F$ ratio and $L F$, which is associated with increased stress, decreased attention and boredom. Some subject has expressed boredom during the fishing game, they could not engage properly with the game because it wasn't challenging or exhilarating. (Jang el al.,2015) revealed that boredom occurs due to the perception 
that there is nothing to do and their surrounding is boring, it is considered an unpleasant and transient (lasting for a brief moment), resulting in a decline in cognitive attention [92]. Boredom may lead to irritation, mental strain and anxiety (which are all preceded by stress), which would correlate to increase in $L F, L F / H F$, decreased HRV and attention.

\section{Chapter 4}

\section{Classification of Stress Through Machine Learning}

\subsection{Introduction}

ML is a term used to represent various computerized models which can be utilized to interpret patterns associated with certain data and is capable of making automatic decisions, coding is not required to lead to the outcome [94]. ML models learn from patterns/past experiences associated with the data, which is represented through structures, similarities and dissimilarities [994]. Patterns explain and indicates the probability associated with the classification of an outcome [94]. ML tools are prominent for automatic classification of probabilities associated with certain outcomes due to common trends and patterns integrated within the data [94]. There are various ML models which can be utilized for a wide variety of classification problems. Some models are more efficient for certain problems in comparison to others, a specific model should be selected based on data specifications, and composition of the problem [94]. Stress is evaluated through 4 distinct processes: identification/detection, classification, quantification/estimation and prediction. Data engineering and cleaning (preprocessing) through established methods such as clustering, and reduction is crucial for a successful outcome [94]. There are numerous mathematical models and algorithm which has been established in order to predict and classify outcomes, but past decade has revolutionized the application of mathematical model's through machine learning [93]. This provides computers the ability to learn certain data associated with CVD, stress, myocardial infarction, . [93]. Recent advances in medicine incorporates machine learning to classify health impairment, which allow clinicians to diagnose diseases at a faster rate 
[93]. Researchers are able to analyze large/complex dataset in a small period, Isaac R Galatzer et. A1. [93] conducted an experiment to discriminate between chronic PTSD (post-traumatic stress disorder) and non-PTSD through machine learning, Zheng at. Al. [93] were able differentiate/classify emotions through deep network using EEG features.

For this experiment, the data size was fairly small since it only included 15 subjects. A dataset with 50 samples and 18 features derived from ECG were utilized to classify stress. Machine learning algorithm was utilized to classify between stress and non-stressed subject due to virtual reality roller coaster simulation, cognitive color stroop task and VR fish game. There were 13 samples from baseline phase, 13 samples from VR roller coaster simulation phase, 13 samples from cognitive color stroop task phase and 11 samples from VR video game phase. The HRV features extracted were $H R$, $S D N N, N N 50$, $p N N 50, R M S S D, S D 1, S D 2$, ApEn from time domain. From frequency domain $V L F, L F, H F, T P$ and $L F / H F$ ratio were extracted through $\mathrm{AR}$ and Lomb Scargle periodogram method. Data was preprocessed using minmax scaler, normalizer, binarizer or standardize. Selected features were labeled as stressed or not stressed, 0 representing non stressed subject while 1 was used to represent stressed subject. The data was classified using the various methods mentioned above, the results are presented through box plot and correlation matrix in order to understand which features play an important role in inducing stress. The TP, $F P, T N, F N$ associated with classification accuracy of the model is indicated through the confusion matrix. $A U C, F 1$, precision and recall results further validate the true accuracy of the model. The experiment indicates the probability of classifying stress through wireless devices, as well as demonstrating the effectiveness of stress detection/prediction through machine learning algorithm. It also hints at future possibilities associated with wireless monitoring of patients suffering from CVD, hypertension and myocardial infarction. Machine learning is utilized ubiquitously such as health care, medicine, finance ., automatic classification can aid the life of millions of people around the world concerning their well-being, physical impairment and economy.

\subsubsection{Supervised Learning/ Machine Learning Models:}

\section{Naive Bayes}

A generalized approach is often utilized to classify vectors with discrete-valued features $\mathrm{x} \in$ $\{1 \ldots, K\}^{\wedge} D, K$ represents the number of values associated with the corresponding feature, $D$ 
represents the feature themselves [96]. Assuming that the features are conditionally independent, class of condition density can be represented as a product of one-dimensional densities

$$
p(\mathbf{x} \mid y=c, \boldsymbol{\theta})=\prod_{j=1}^{D} p\left(x_{j} \mid y=c, \boldsymbol{\theta}\right.
$$

which represents the Naive Bayes classification model [96]. The features utilized using Naive Bayes model are often not independent, it is a simple model (with $O(C D)$ parameters, $C$ represents classes and $D$ represents features) which also makes it resistant to overfitting [96]. Class conditional density is specific to the feature utilized, Gaussian distribution is often used for real valued features, Bernoulli distribution is used for binary features and Multinoulli distribution is used for categorical features [96]. Evaluation $M L E$ and $M A P$ estimates of the parameters is required to train the model.

\section{Decision Tree}

Decision tree were implemented through separation of specific features from a random subset of $K$ feature within each internal node. The most efficient group is split from the randomly distributed feature, which is determined from the Gini impurity index associated with each feature. It is capable of predicting outcomes through small decisions made from variations associated with the features. It consists of nodes which was dictated through a decision, it results in a leaf which indicates the last class. Decision tree are very simple and easy to interpret, present a smart and facile visual representation, DT can lead to overfitting due to model complexity.

\section{KNN}

K-Nearest-Neighbor (KNN) is a very simple and highly efficient pattern classification/regression model, it is also regarded as a prominent supervised machine learning model used for classification problems associated with data mining [83]. It was developed from the distance which was used to measure similarity/dissimilarity between two samples [83]. Given a training dataset which includes the features and labels, KNN algorithm can efficiently learn the data and predict labels for a certain circumstance [83]. Given function $d(x, y)$, the Euclidean distance between $\mathrm{x}$ and $\mathrm{y}$ would be computing using the following equation:

$$
d(x, y)=\sqrt{\sum_{i=1}^{n}\left(a_{i}(x)-a_{i}(y)\right)^{2}} \quad-1
$$


KNN includes nearest neighbors which classifies an instance based on similarity, closest points to the nearest neighbours indicates the group which is classified using the algorithm [83].

\section{LDA}

Linear Discriminant Analysis is commonly regarded as a dimensionality reduction method, it is capable of reducing the number of dimensions within a dataset while retaining most of the information, it limits the reduction of valuable information as much as possible.

\section{SVM}

Support vector machine are utilized to find the hyperplane within the N-dimensional space which can discriminate between the corresponding classes associated with $\mathrm{N}$ number of features.

\section{Ensemble Gradient Boosting Classifier}

Ensemble Gradient boosting classifier is strong learner which is developed from ensemble of weak prediction models consisting of decision trees, and it is ideally utilized for regression and classification. This model also included hyperparameters which further optimized the efficiency of the model. It was embedded with a learning rate parameter which allowed the model to automatically configure itself based on the error estimated. This allowed the model to mimic a neural network. A process flow used to describe the function of gradient boosting classifier is displayed below on Figure 53.

\subsubsection{Preprocessing}

\section{Rescale Data}

Data which includes features with different scales, rescaling can result in the same scale, normalizing the data between 0 and 1. It's often utilized for optimization algorithms such as gradient descent, it is very efficient for regression algorithms such as K-Nearest Neighbour, Neural Networks which use weighted inputs, distance. MinMaxScaler can be used to rescale the data using scikit-learn.

\section{Standardize data}

Standardization is a very effective technique which can efficiently transform data with a Gaussian distribution to be between 0 and 1. It's a great method to complement rescaled data and 
works effectively to transform regression methods such as linear regression, logistic regression, and linear discriminant analysis.

\section{Normalize Data}

Normalizations rescales each row into 1, it's an effective method for scattered data which includes many zero values and different scales. K-NN and NN which weight input values will find this method applicable to their data.

\section{Binarize Data}

Binarize method transforms values from a dataset into 1 if the value is above a certain threshold and 0 if it is below a certain threshold. It makes probabilities look more attractive, effective for feature engineering and adding new features. Binarizer is used to transform the dataset.

\subsubsection{Feature Selection}

\section{Univariate Selection}

Univariate selection is based on statistical test such as chi-squared which selects the features based on the strongest relationship with the label. SelectBest class 2 from scikit learn library can be used to select specific features based on different types of statistical tests.

\section{Recursive Feature Elimination}

RFE is used to recursively removes features, and then generates a model with the remaining features. Model accuracy using a certain model such as LRA, is used to identify the best features, RFE class 3 is the algorithm utilized for this purpose. Principal component analysis is often utilized to predict labels.

\section{Principal Component Analysis}

PCA transforms the data into compressed data through linear algebra, it is a reduction technique but the number of principal components (important features) after transformation is dictated by the user.

\section{Feature Importance}

Random Forest, Extra trees are types of decision trees which can assess feature importance, decision tree classifier such as ExtraTreesClassifier class5 from scikit-learn API. 


\subsection{Method}

\subsubsection{Process Flow Chart}

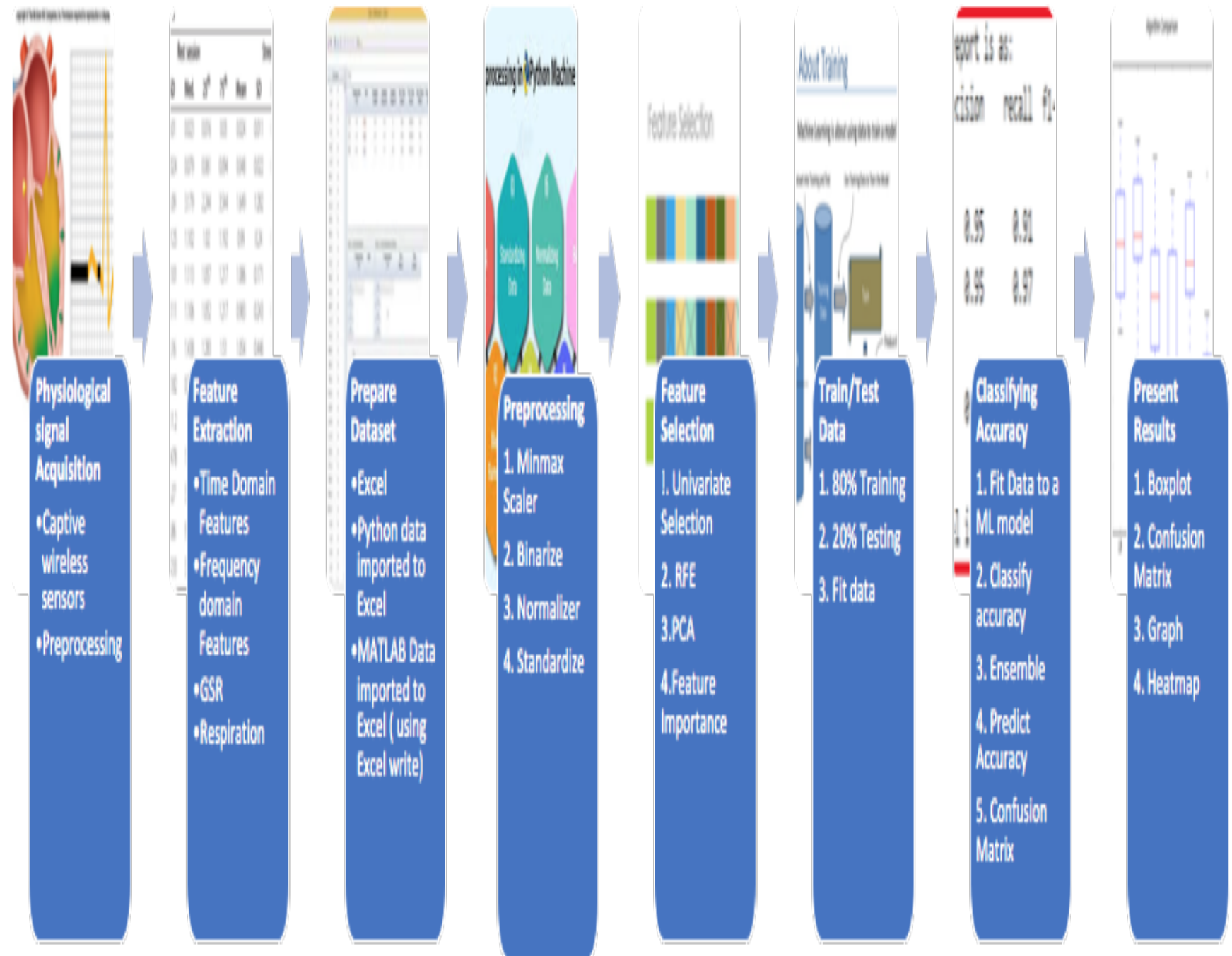

Figure 56: Procedure used to classify stress/relax after data acquisition. 


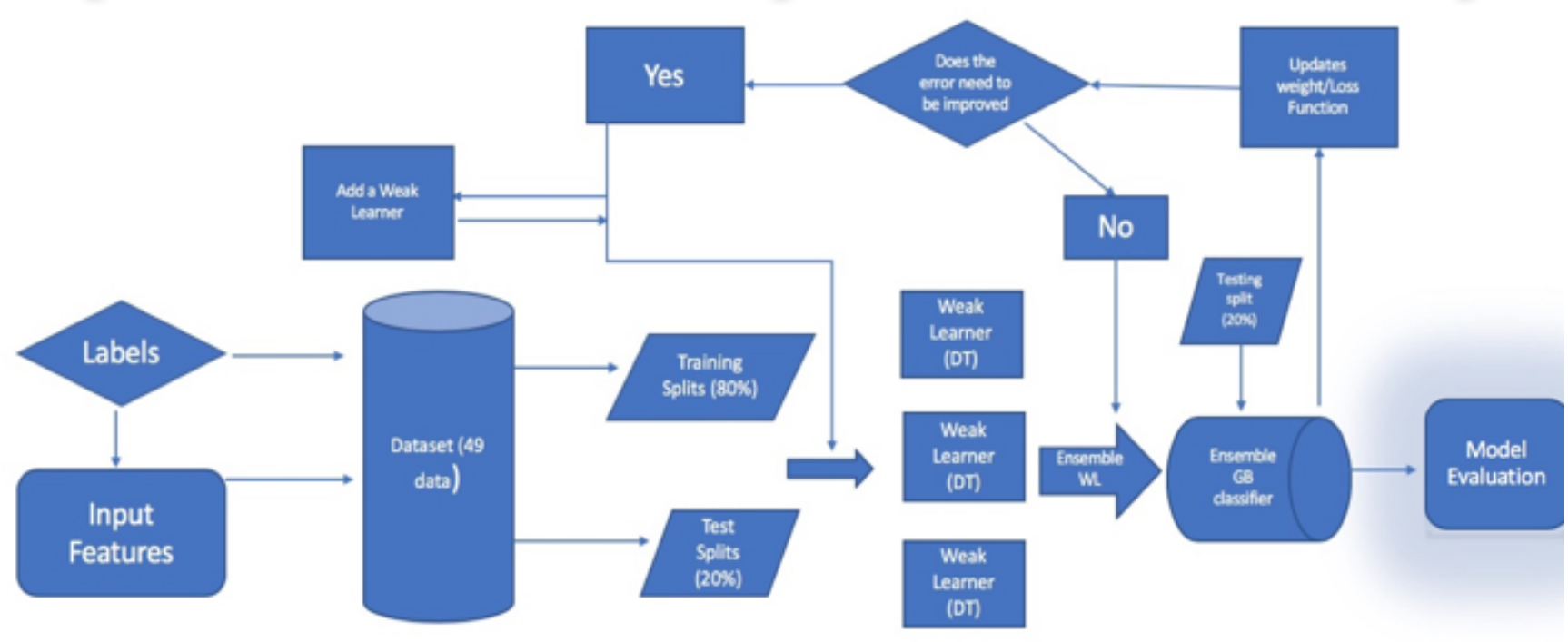

Figure 57: Process flow chart used to derive Ensemble Gradient Boosting classifier and evaluate the model.

\subsubsection{F1, Precision, Recall, Auc}

$$
\text { Precision }=\frac{\text { True Positive }}{\text { True Positive }+ \text { False Positive }}
$$

Precision examines the precision and accuracy of the model, indicating the $T P$ out of the total which includes both $T P$ and $F P$.

$$
\text { Recall }=\frac{\text { True Positive }}{\text { True Positive }+ \text { False Negative }}
$$

Recall is used to identify the amount of TP collected through the model.

$$
\mathrm{F} 1=2 \times \frac{\text { Precision } * \text { Recall }}{\text { Precision }+ \text { Recall }}
$$

F1 represents the test accuracy associated with the model. High F1 is associated with a good precision and recall score.

$R O C$ (receiver operating characteristics) represents a probability curve, true positive rate represents the $\mathrm{y}$ axis, false positive rate represents the $\mathrm{x}$ axis. 
$A U C$ (Area under the curve) indicates how well the model can distinguish between the labels/classes. A high $A U C$ can discriminate between positive and negative class (stress, relax) with high accuracy.

\subsection{Results}

The experiment was separated into 4 phases, beginning with a baseline phase to monitor their baseline physiological function and ending with a VR fishing game which was meant to relax them. Stress induced through the VR roller coaster phase and cognitive color stroop task was unique for each subject. Some subjects were highly imaginative and unable to withstand the roller coaster phase without any movement, while others felt it was quite enjoyable. The level of stress induced within each subject varied anywhere from low/normal stress level (they liked the simulation) to high level of stress (which caused the subject to sweat heavily and become emotional). This section presents ML models and methods used to classify and analyze binary stress and relax classes. 


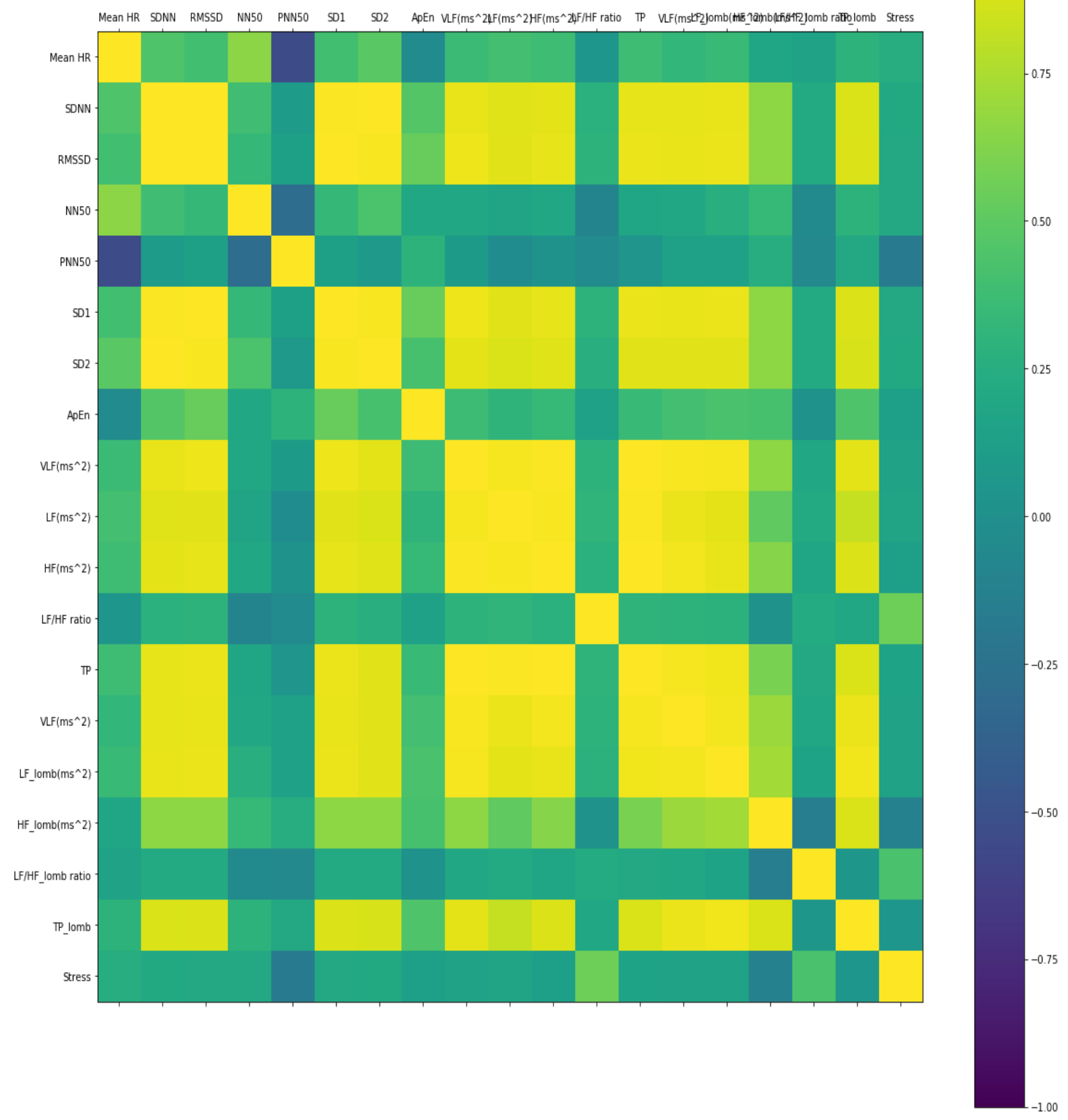

Figure 58: Correlation map to understand which feature were the most significant for classifying stress 
Figure 58 displayed above describes the correlation matrix of the 18 features and stress label. $L F / H F$ ratio obtained from lomb scargle periodogram PSD is an important feature in classifying stress, and shares around 0.75 correlation with stress which is indicated through light green color. The value is positive which indicates that increase in $L F / H F \_$lomb ratio also results in an increase in stress. Another $L F / H F$ ratio was obtained from AR model, it is also positively correlated to stress. This feature is a slightly lighter shade of green in comparison of $L F / H F$ lomb ratio, which indicates that it shares slightly higher correlation with stress which may be represented by values ranging from $0.75-0.80$. Similarity this feature is proportional to stress, increase in ratio will result in an increase in stress. HF lomb obtained from Lomb scargle periodogram is somewhat bluish and green, from the scale it shares a -0.25 correlation with stress, the negative correlation indicates that the feature is inversely proportional to stress, increase in $H F$ value will result in reduced stress and vice versa a decrease will result in increased stress levels. Another feature PNN50, which is a time domain feature is represented by a more solid blue color. It probably shares a -0.50 correlation with stress, similar to $H F$, this feature is also inversely proportional to stress, a decrease in PNN50 will result in an increase in stress.
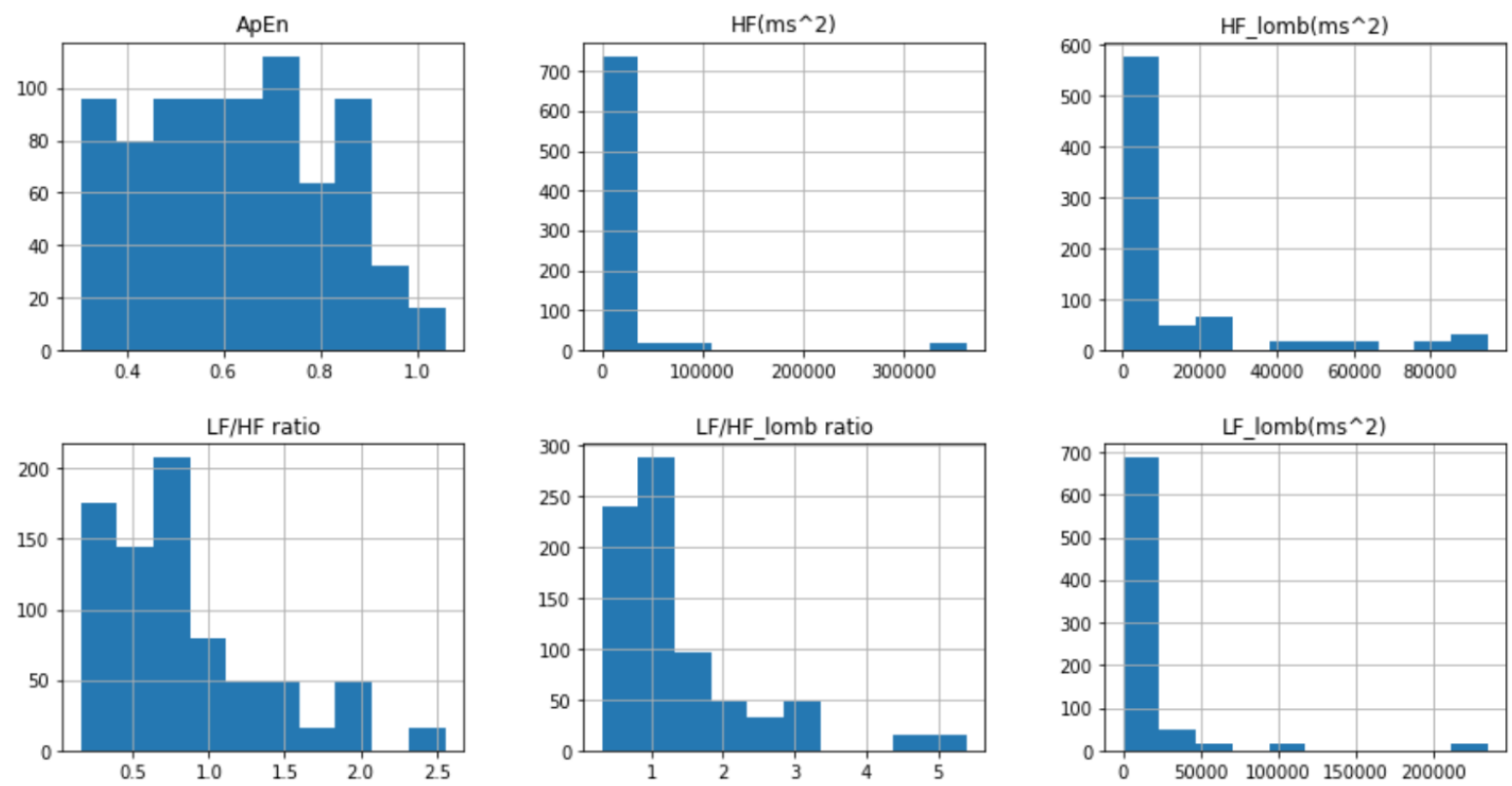

Figure 59a): Histogram of the Features used to classify stress through binary classification 

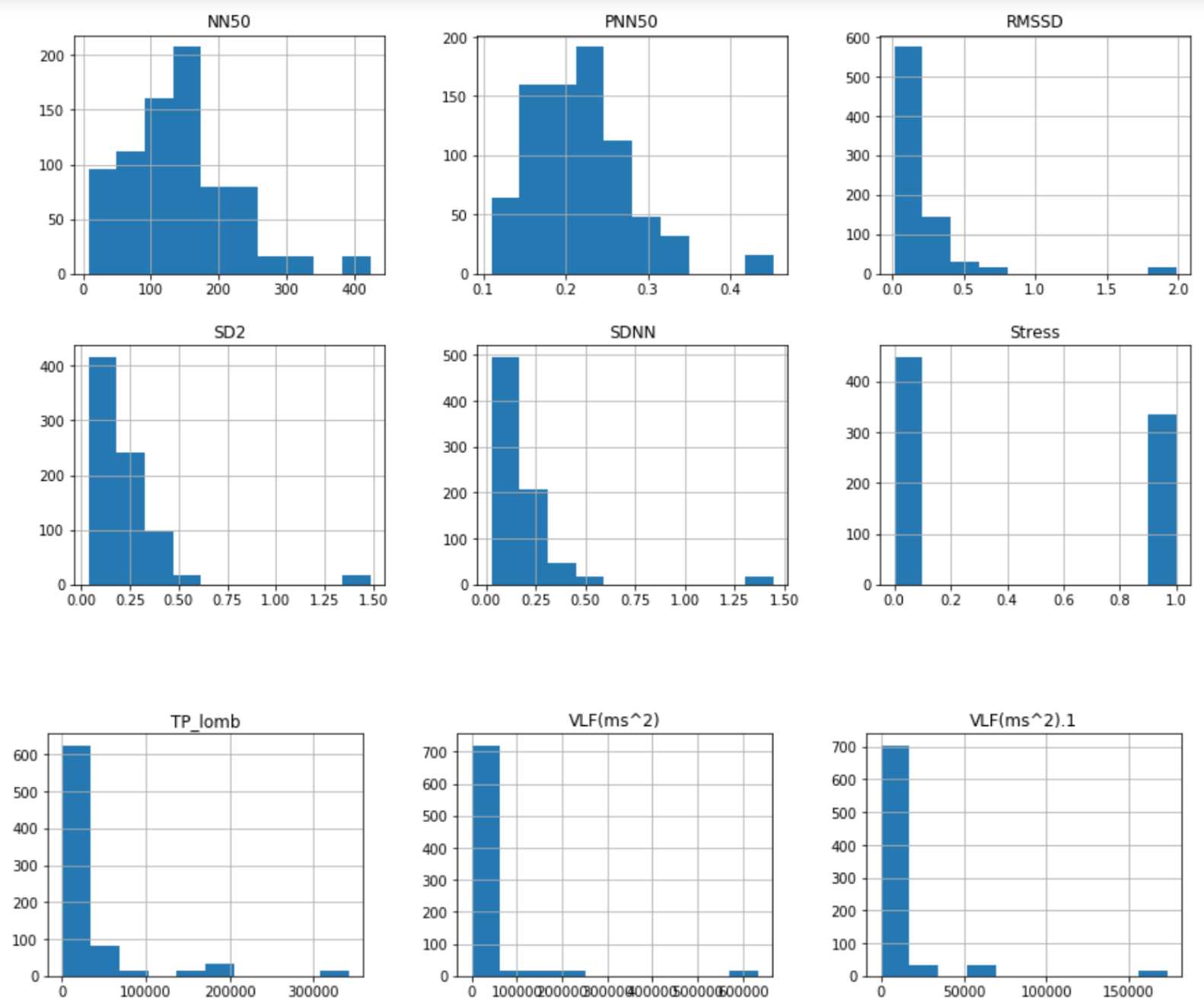

Figure 59b): Histogram of the Features used to classify stress through binary classification 

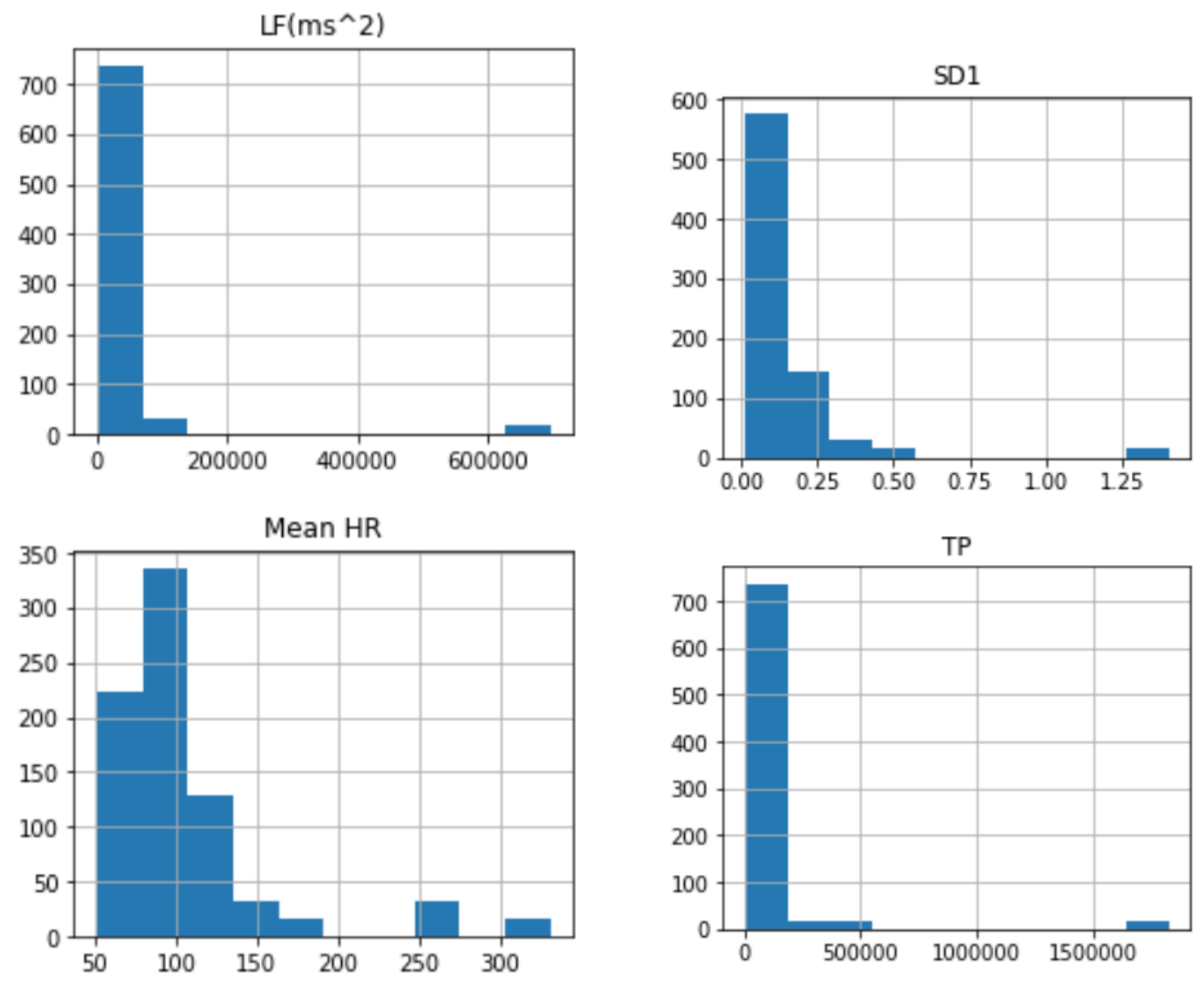

Figure 59c): Histogram of the Features used to classify stress through binary classification

Figures 59(a, b, c) displays the histogram of the features used to classify whether the subject was stressed/relaxed. It is an accurate representation of the distribution of values associated with the features and the corresponding label. 

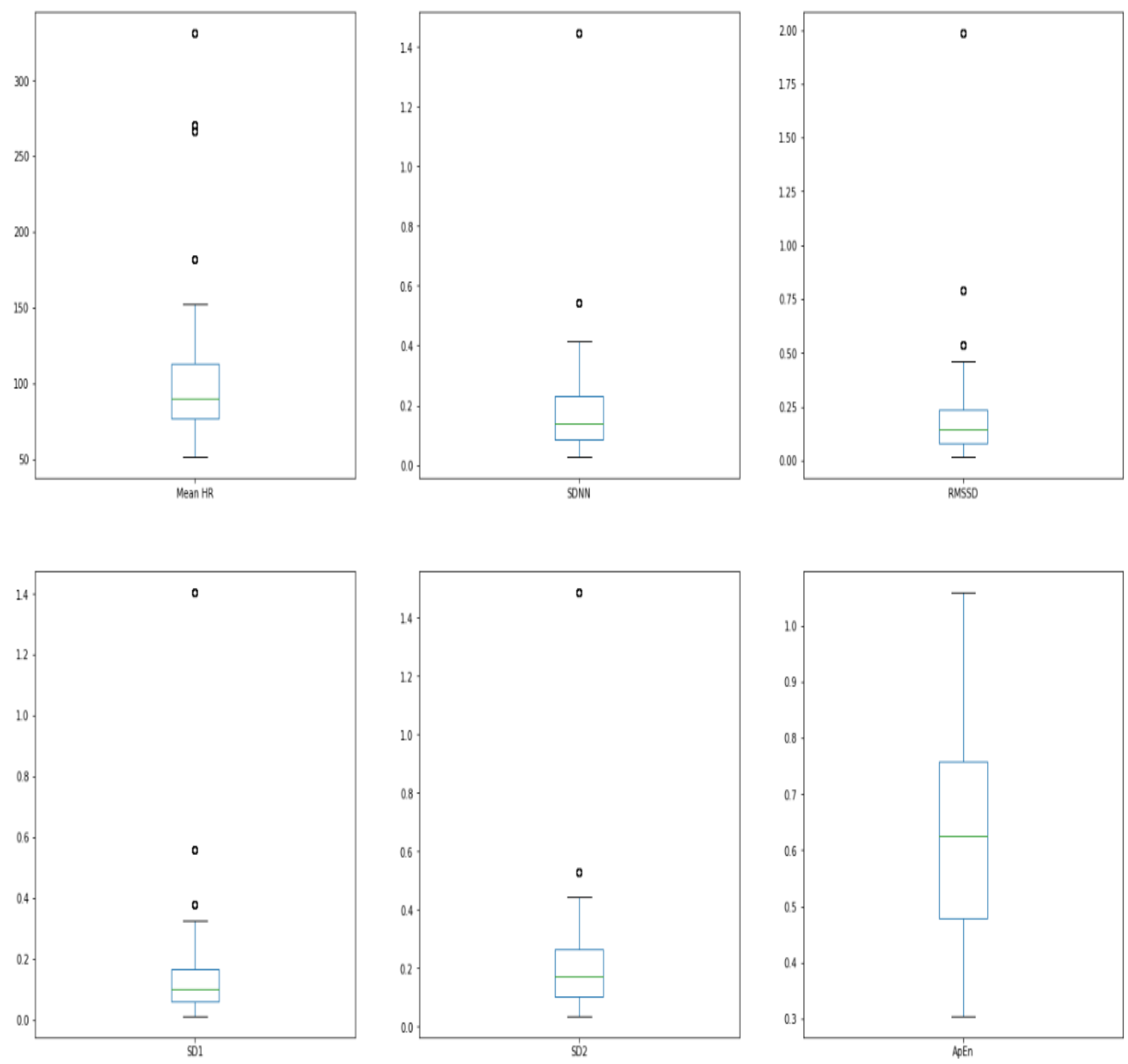

Figure 60: Box plot of features and stress labels used for binary classification 

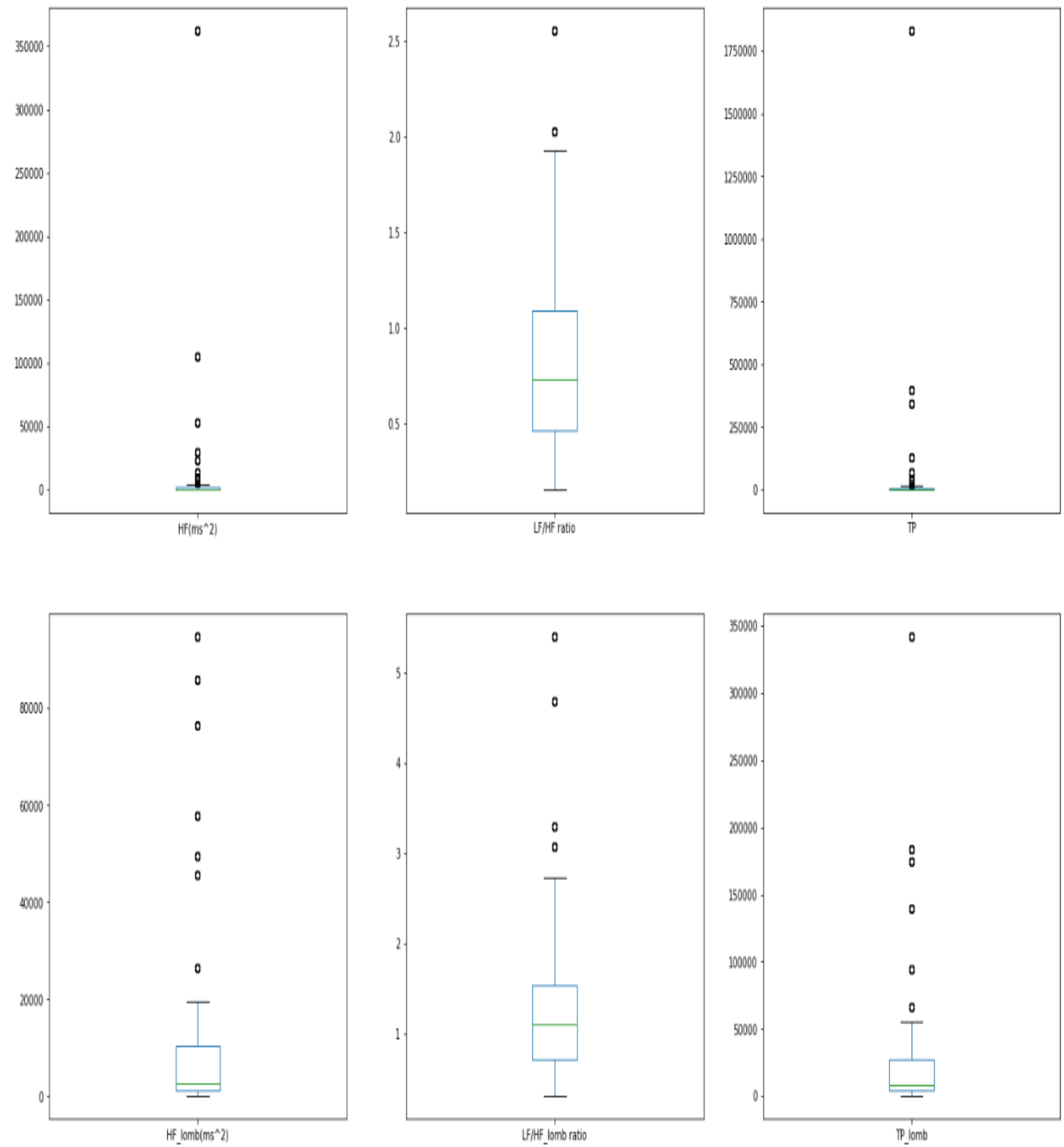

Figure 61: Box plot of features and stress labels used for binary classification 

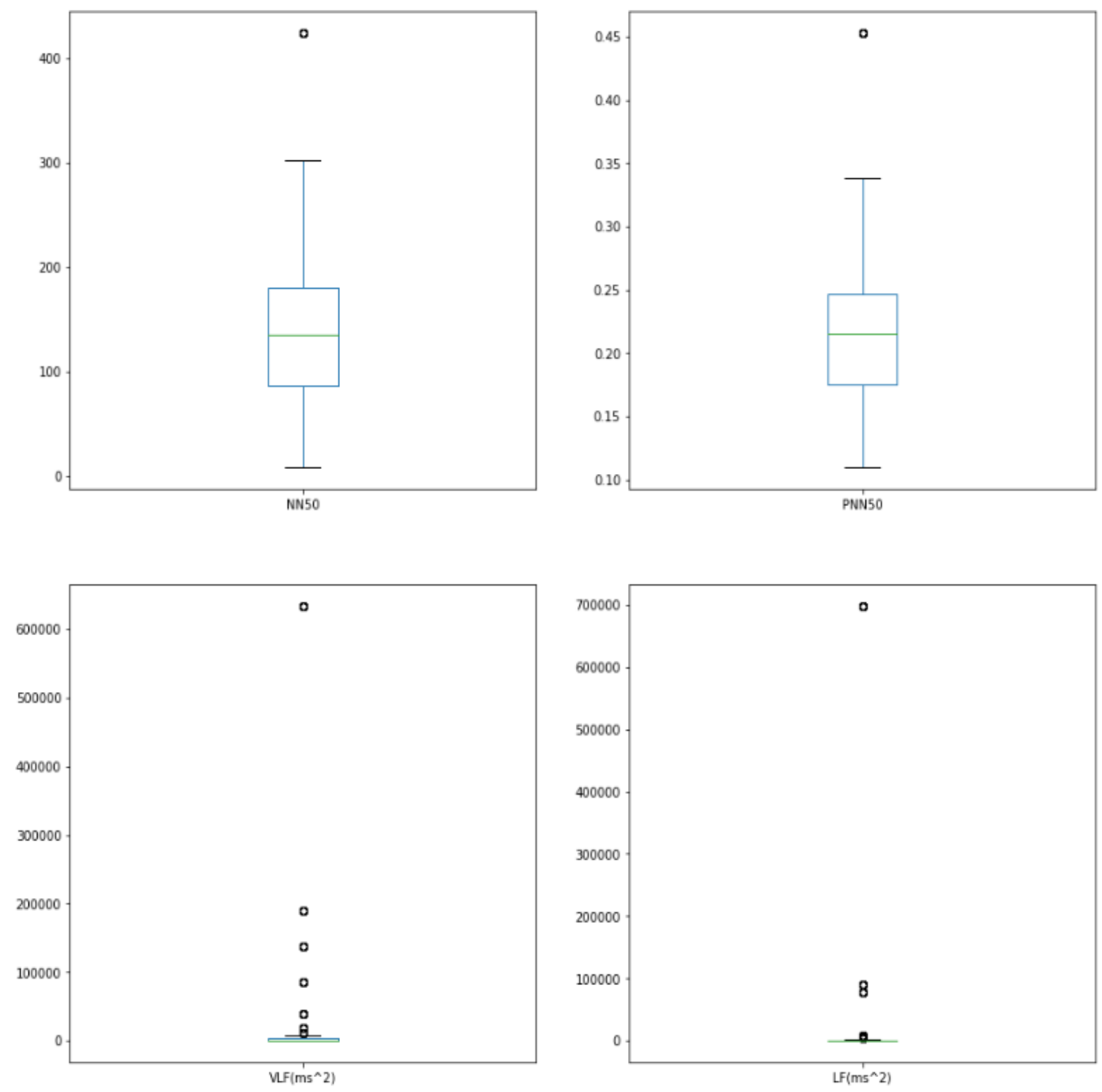

Figure 62: Box plot of features and stress label used for binary classification 

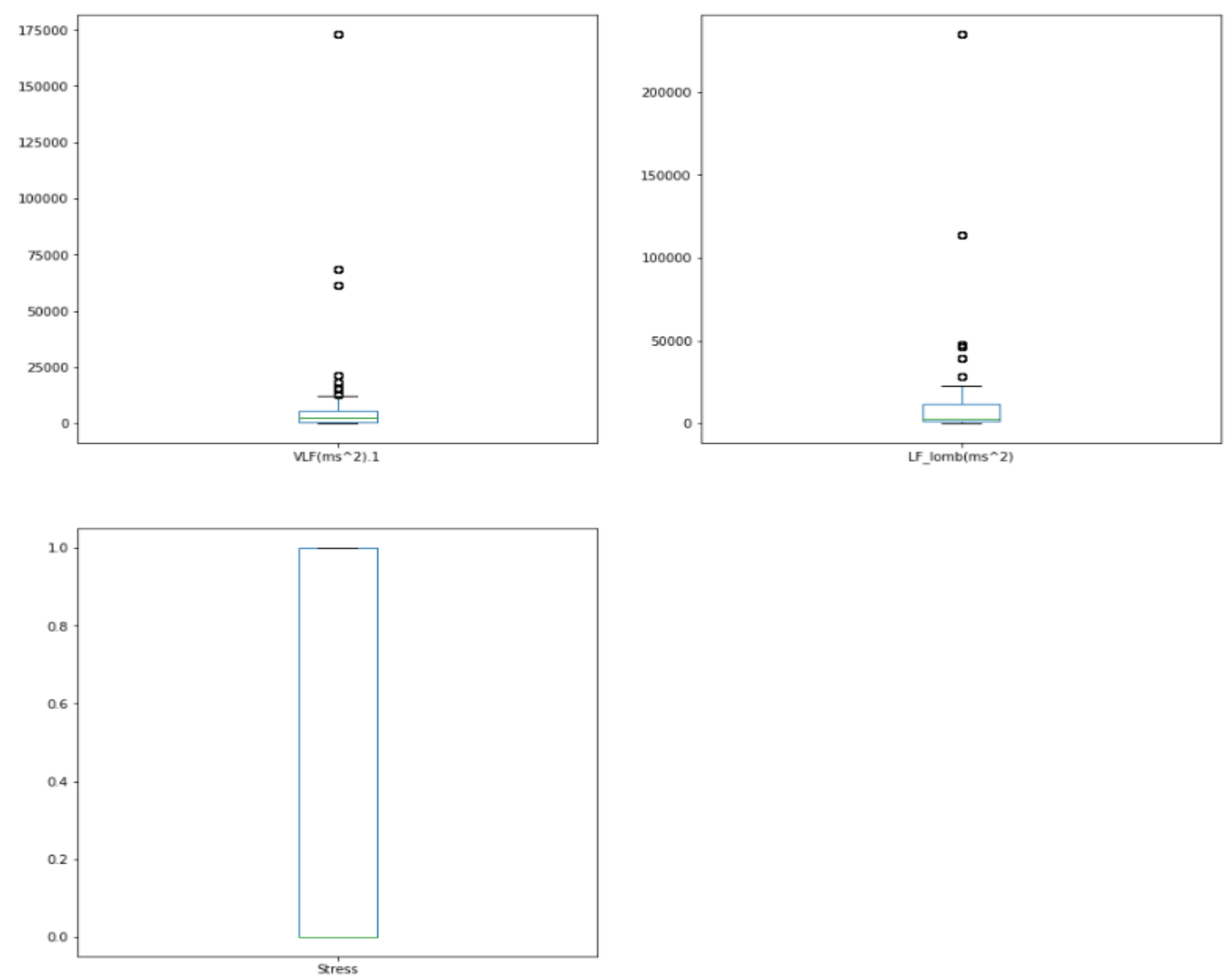

Figure 63: Box plot of features and stress label used for binary classification

Figures 60-63 presents a graphical representation of statistical data associated with each feature and stress labels. Box plot is a visual analysis of data distribution through quartiles, which is based on minimum, median, third and maximum quartile. Median value represents the value in the middle from the highest and lowest distributed values. Three Quartile (lower, upper, interquartile) divide the data into 4 groups. Dots outside the highest quartile represent outliers which significantly differ from the majority of the data. Box plot is capable of presenting large data in an efficient manner through median value, upper and lower quartiles as well as outliers. Box plots complement histogram when presenting a thorough and detailed analysis of the data. Box plots are a visually effective method to present the distribution of the data and it gives an 
efficient indication of the symmetry of the data. It also indicates the variance associated with the data through outliers.

\subsubsection{Confusion Matrix}
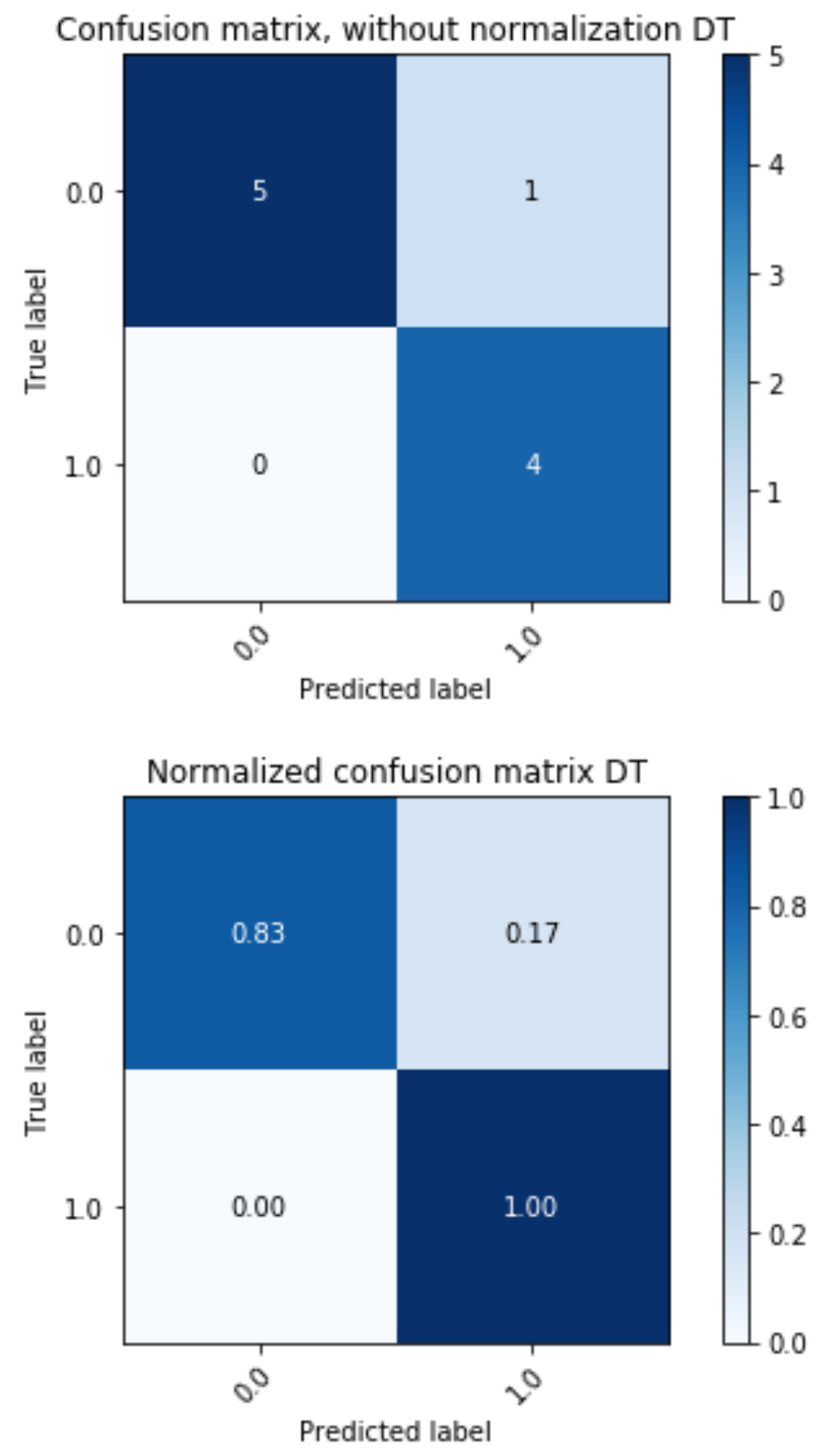

Figure 64: Confusion matrix for binary classification using DT

Figure 64 displays the confusion matrix obtained from binary classification of stress, 5 instances were classified as relaxed and 4 occasions indicated that the physiological function represented 
behaviours of being stressed. DT was able to classify stress with $90 \%$ accuracy, with a $T P$ rate of $100 \%, T N$ rate of $83 \%$, and a $F P$ rate of $17 \%$.
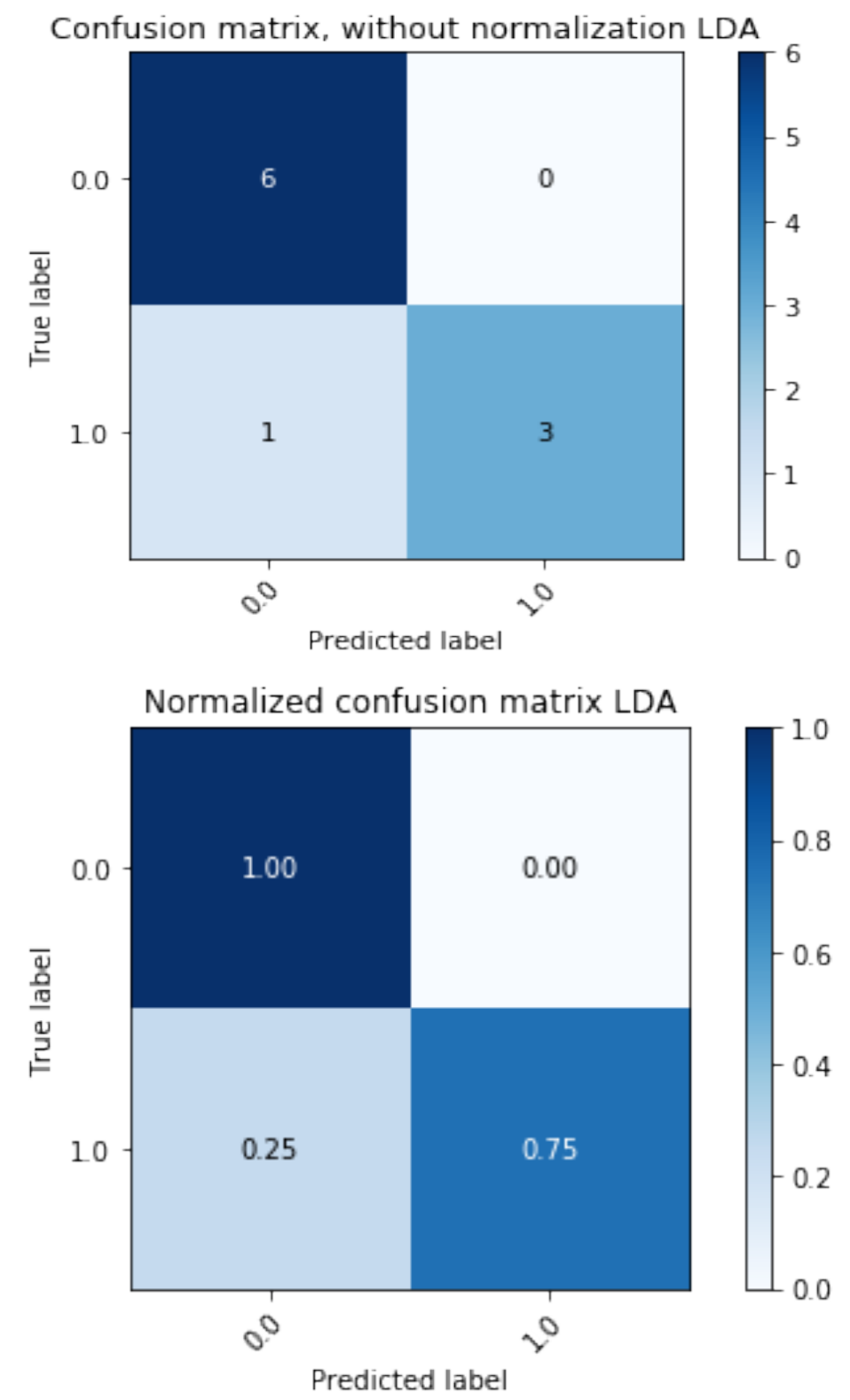

Figure 65: Confusion matrix from binary classification of stress through LDA.

From the confusion matrix in Figure 64, accuracy obtained from binary classification of stress through LDA is revealed. The performance the ML model obtained through LDA indicates that the model produced $T P$ rate of $75 \%$ and $F N$ rate of $25 \%$. The $T N$ rate was $100 \%$. The model had an accuracy of $90 \%$. 

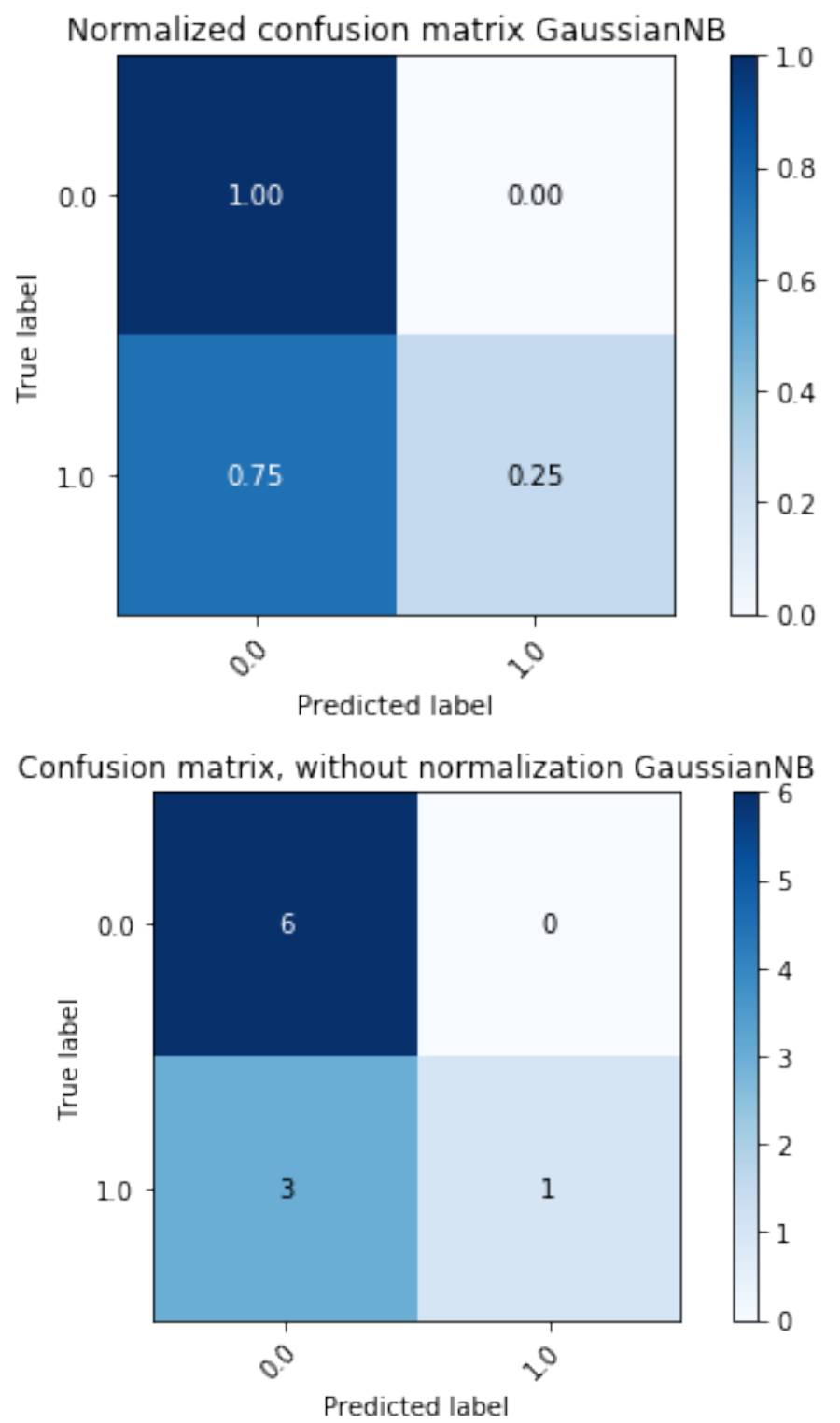

Figure 66: Confusion matrix obtained from binary classification of stress through Gaussian NB.

Figure 66 demonstrated that Gaussian Naive Bayes was not an efficient model to classify stress, the model resulted in a false negative rate of $75 \%, T P$ rate of $25 \%, T N$ rate of $100 \%$. The model achieved a classification accuracy of $70 \%$. 

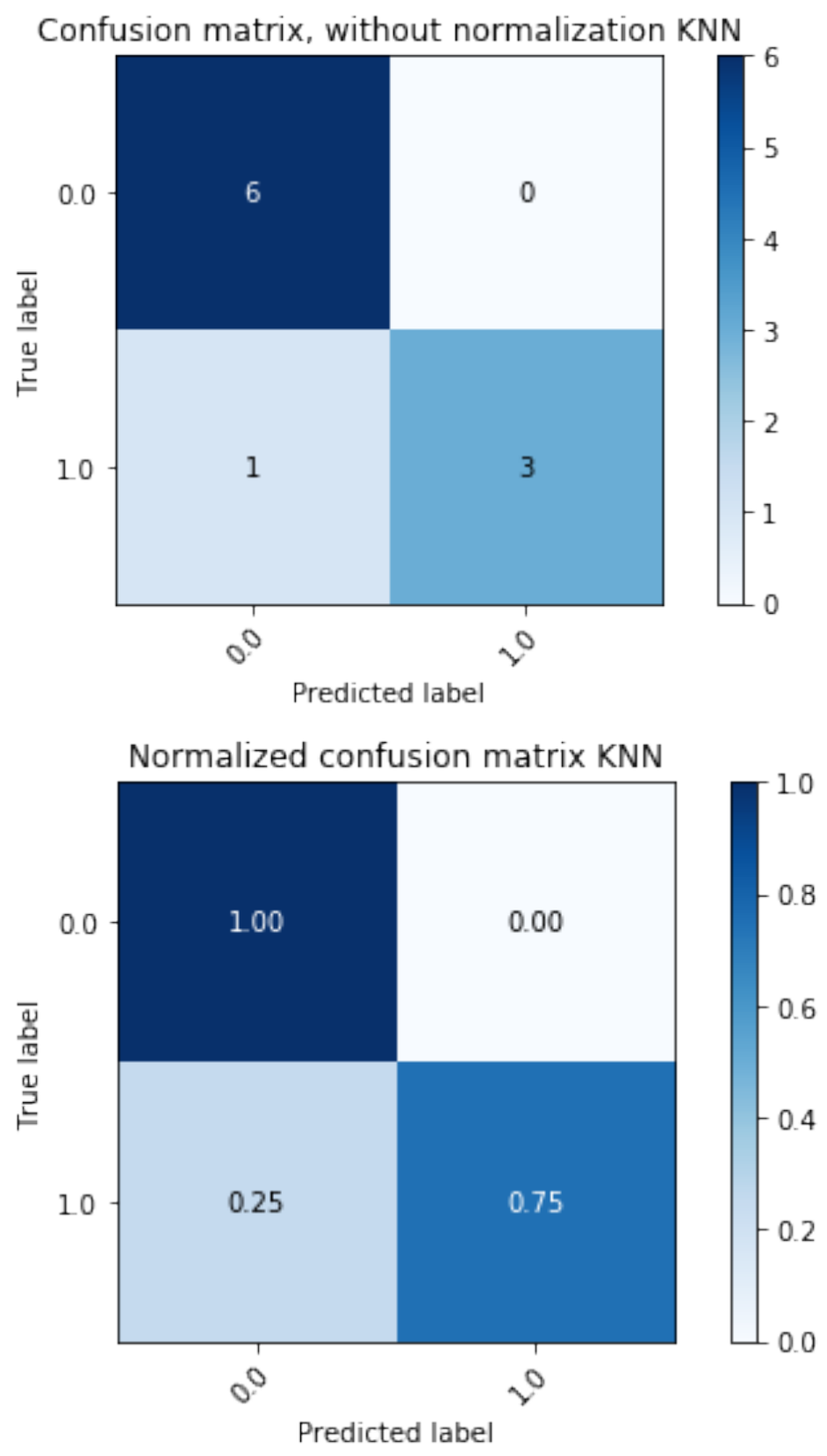

Figure 67: Confusion Matrix of binary classification obtained through KNN

Figure 67 displays the confusion matrix obtained from binary classification of stress using KNN model, the model has a $T N$ rate of $100 \%, T P$ rate of $75 \%, F N$ rate of $25 \%$. 

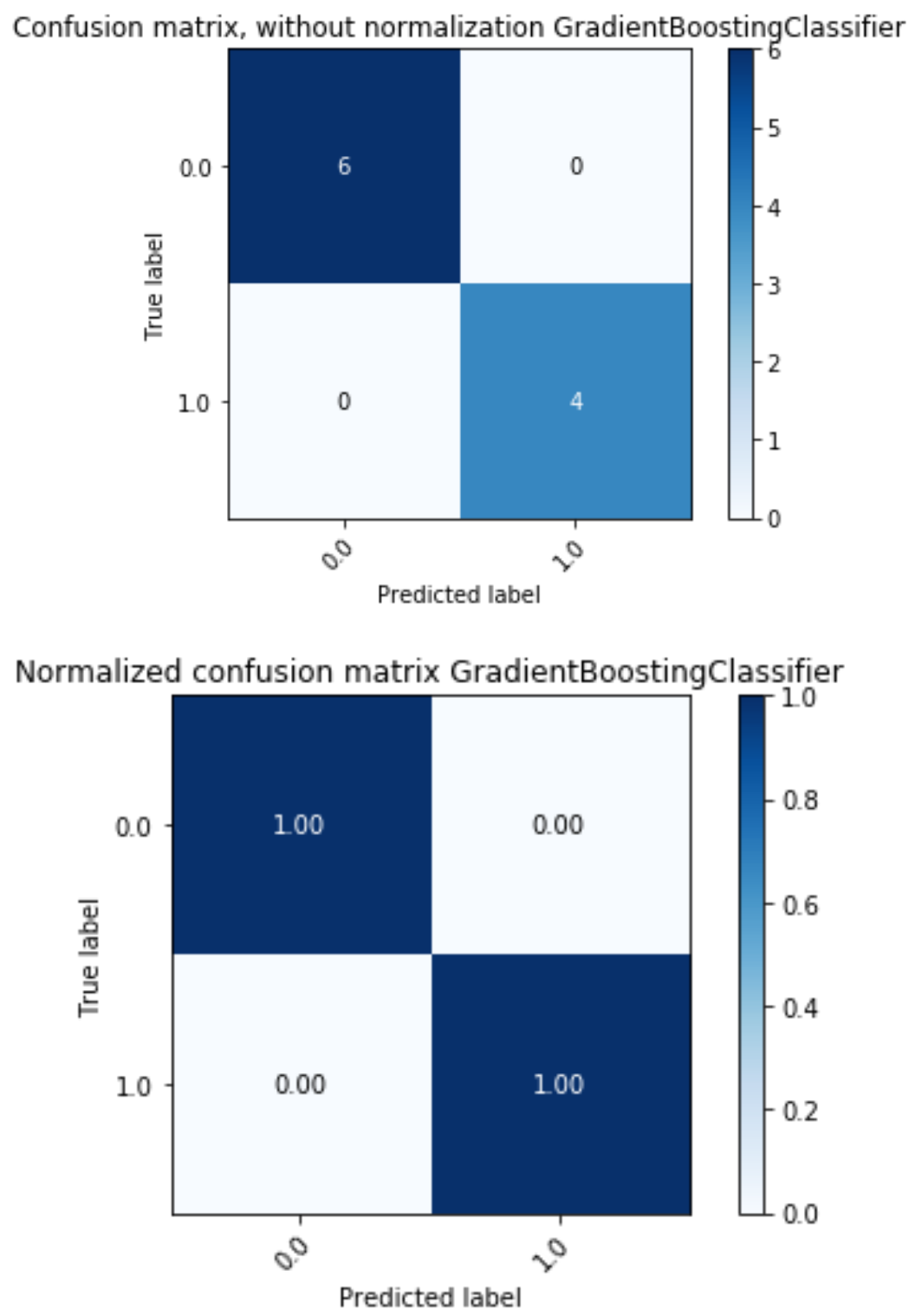

Figure 68: Confusion matrix obtained through ensemble Gradient Boosting Classifier

The ensemble GradientBoostingClassifier model displayed in Figure 68 resulted in a TP rate of $100 \%, T N$ rate of $100 \%$.

\subsubsection{Precision/Recall curves}




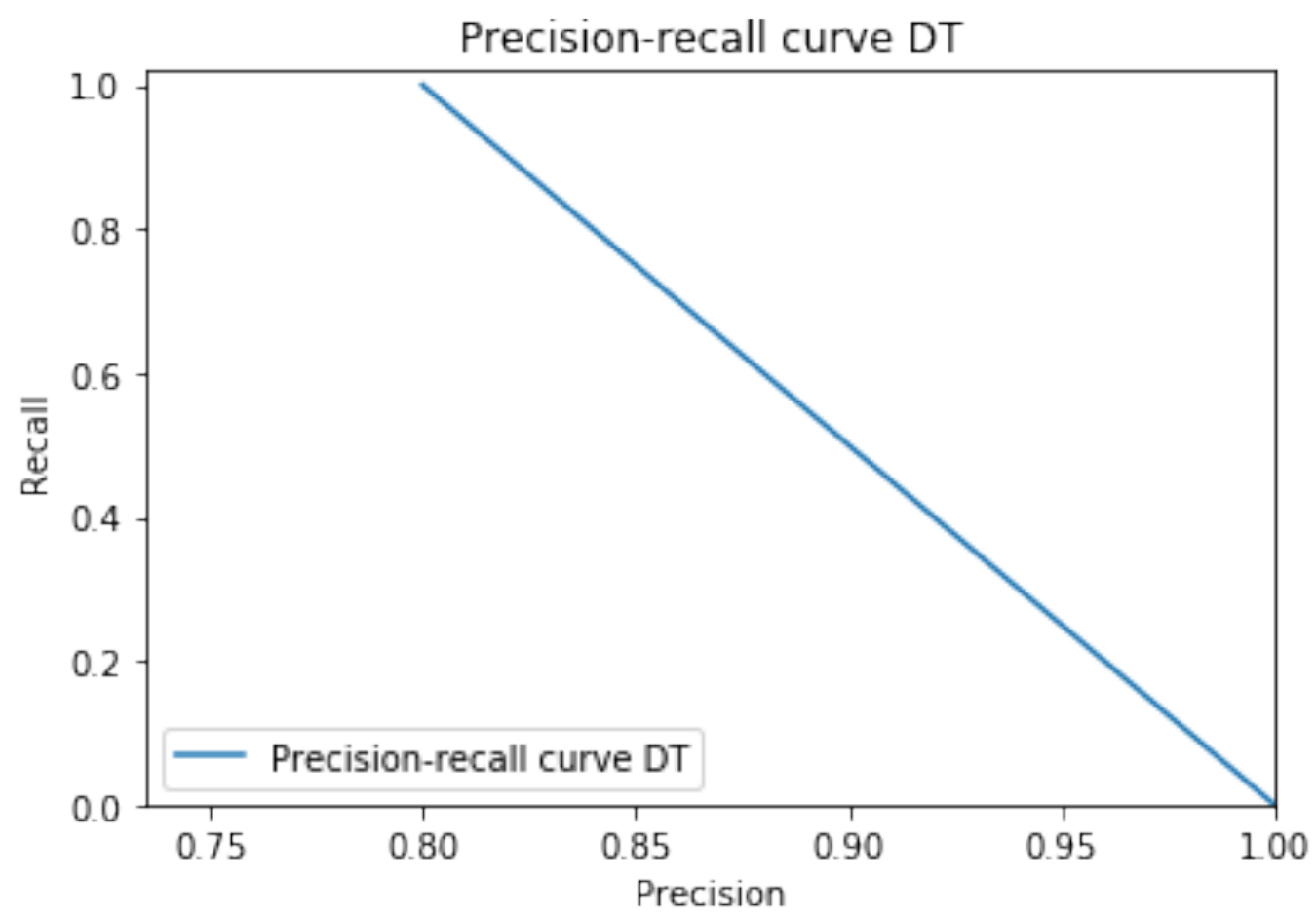

Figure 69: Precision and recall curve for binary classification of stress using decision tree

The Figure 69 displayed above describes the precision- recall curve obtained from classifying stress through binary labels (relaxed, stressed) using DT model. The model achieved an accuracy of $90 \%$ which is indicated by the blue line towards the upper left corner. DT model achieved $100 \%$ precision for relaxed states, and $80 \%$ for stress states. The model achieved $100 \%$ recall for stress states and relaxed class resulted in a recall of $83 \%$, which indicates the classifier accuracy (precision) and the ability to return the detected classified results (recall). 


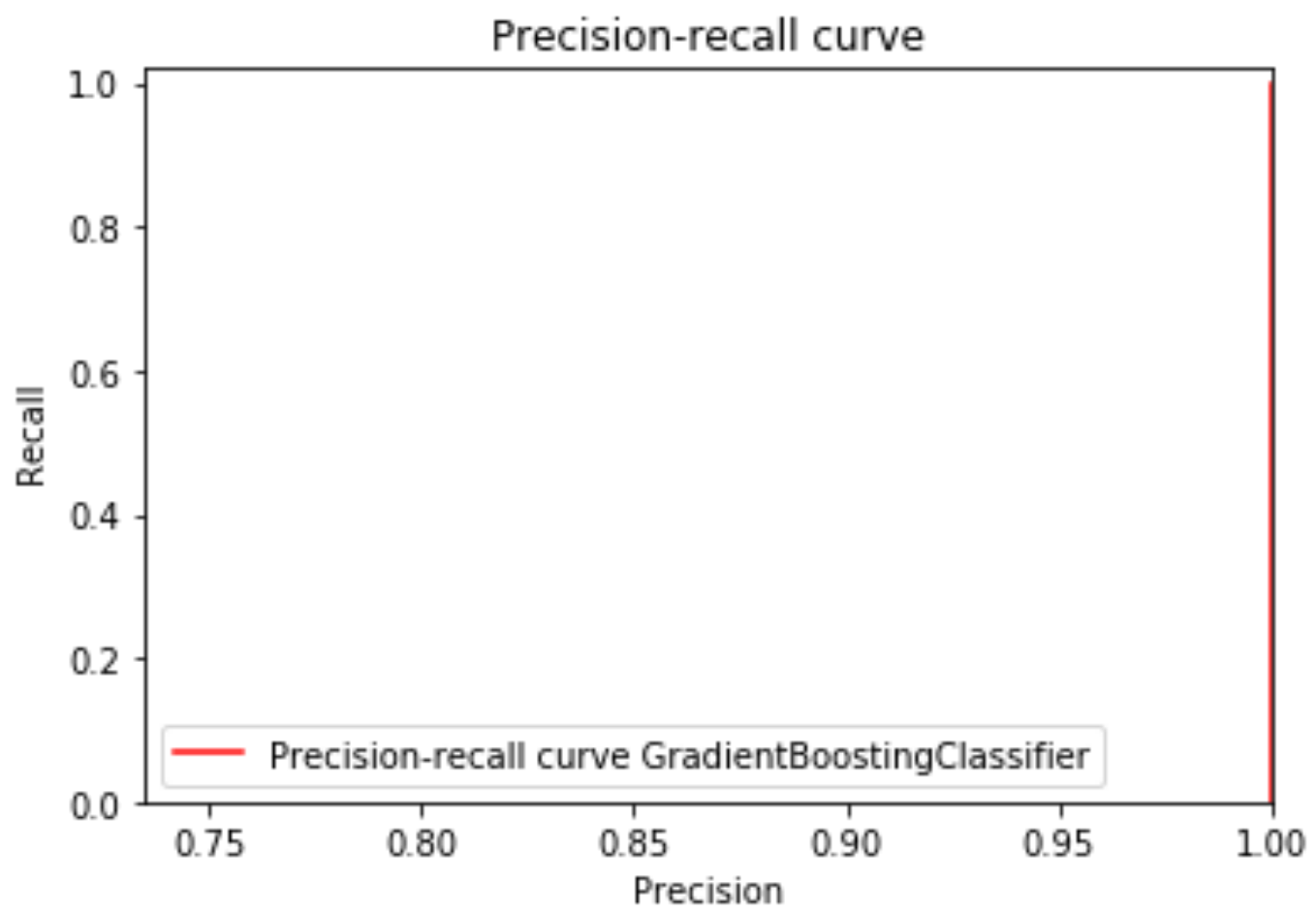

Figure 70: Precision and Recall curve obtained using ensemble Gradient Boosting classifier model (binary).

The previous Figure reveals the precision and recall curve produced from results derived through ensemble Gradient Boosting classifier model. The results indicate that the model produced precision and recall of $100 \%$. 


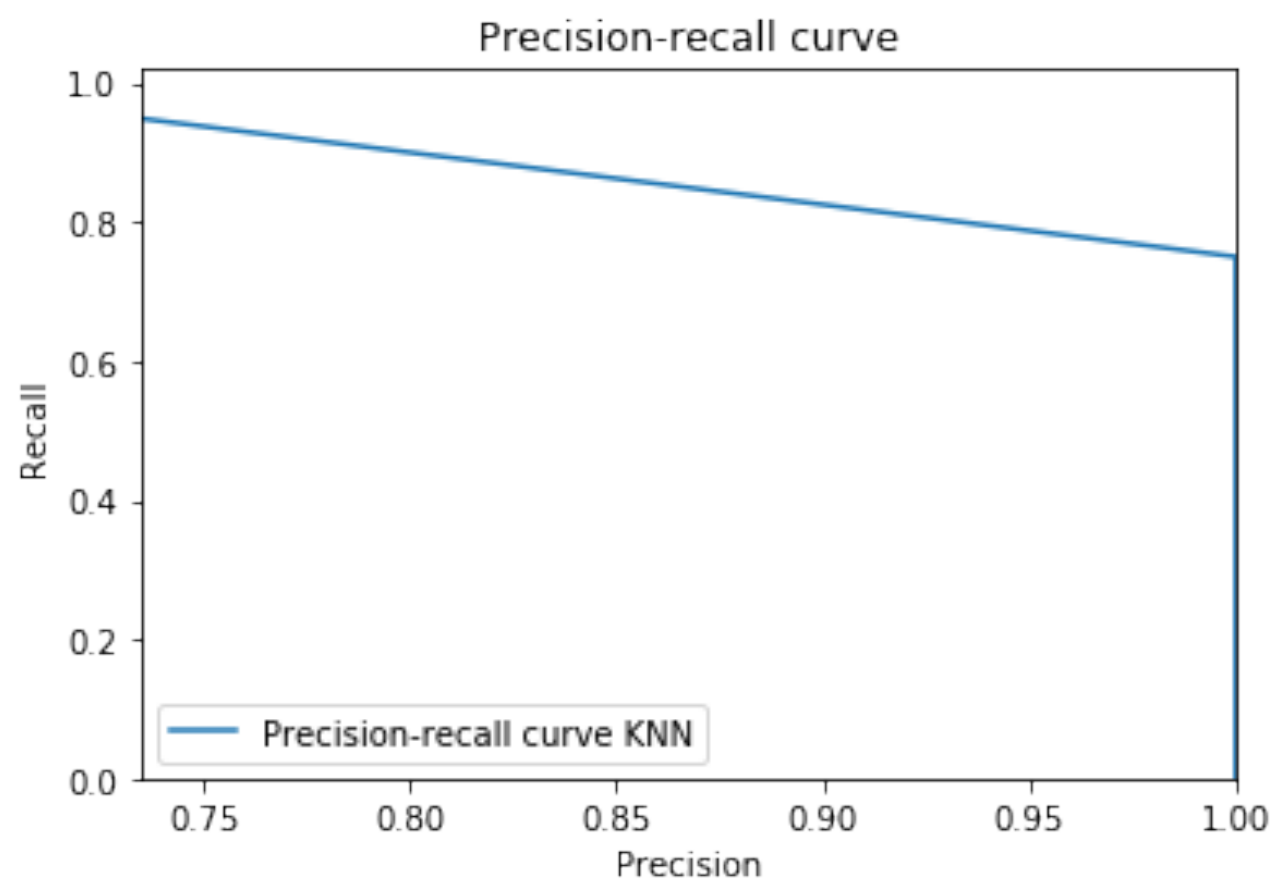

Figure 71: Precision and Recall curve obtained from KNN model (binary)

The preceding Figure 71 presents the precision-recall curve of KNN model during binary classification of stress. The results indicate that when the model achieved $90 \%$ classification accuracy, $100 \%$ of the results were returned for relaxed states, resulted in a recall of $75 \%$ for stress class. The model resulted in a precision of $100 \%$ for stress labels and achieved $86 \%$ precision for relaxed states. 


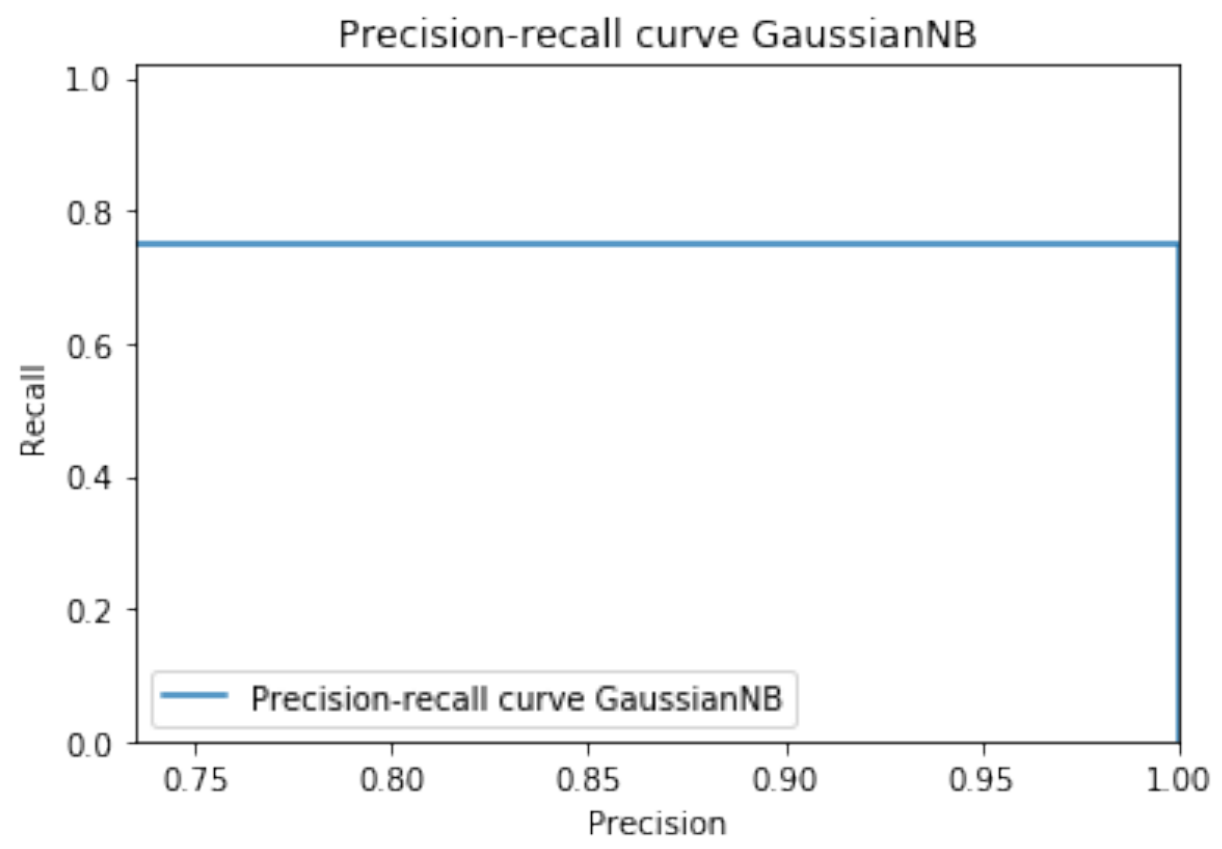

Figure 72: Precision and recall curve of Naive Bayes model (binary)

The former precision and recall curve Figure obtained from NB model indicates that the model was capable of returning $77-78 \%$ of the true positives detected (stress) at $100 \%$ classification accuracy, the model was capable of classifying $T N$ (relax) with 100\% accuracy. 


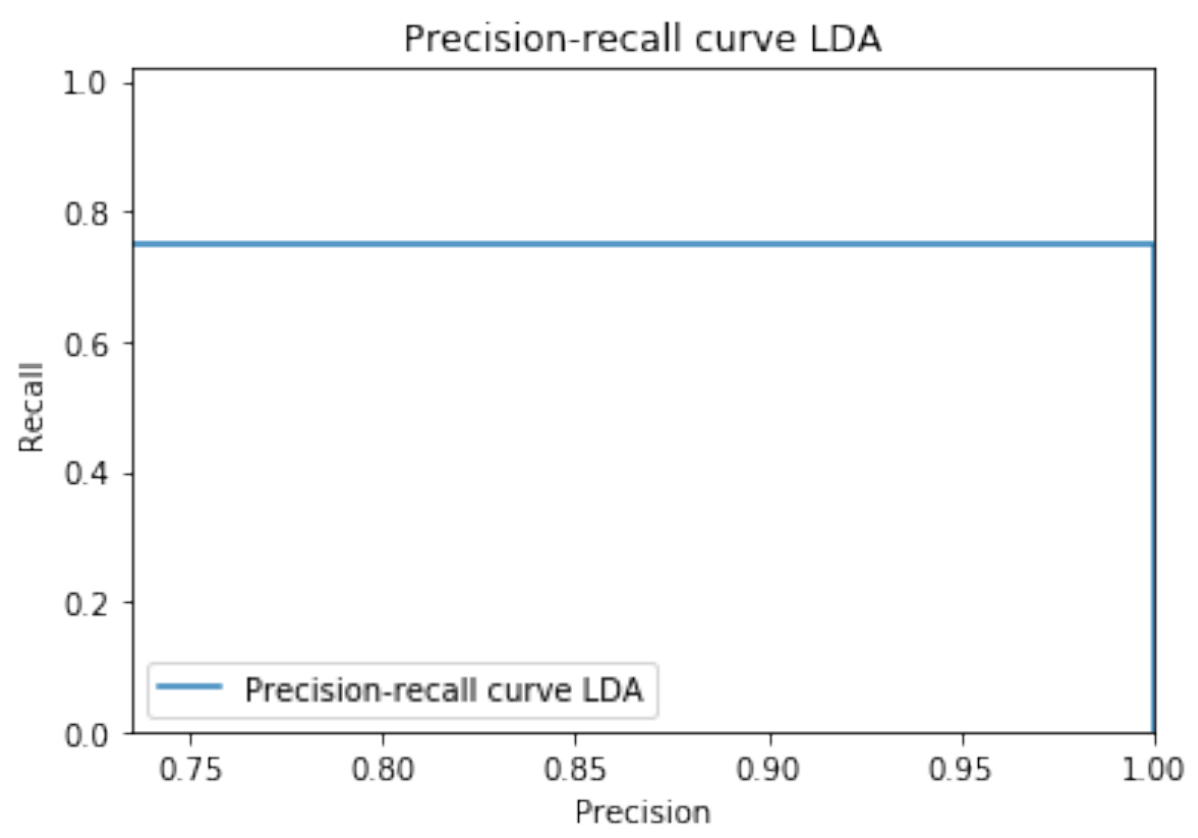

Figure 73: Precision and recall curve from LDA model (binary)

The above Figure 73 represents the precision and recall curves associated with LDA model. The results indicate that the model can return $100 \%$ of TP detected (stress) with $77-78 \%$ classification accuracy.

\subsubsection{ROC curve}

ROC curve presents the sensitivity and specificity associated with stress/relax detection. Sensitivity represents the ratio of total number of true positives with respect to the total number of $T P$ and $F N$, while specificity is the ratio of total number of $T N$ with respect to the total number of $T N$ and FP. Sensitivity represents the accuracy of analysing stress and specificity indicates the classification accuracy of relaxed/normal subjects. The ROC curve is compared with a baseline linear grid line in order to efficiently visualize the performance of the classification model. A good classifier does not interact with the baseline grid line, it is situated towards the top left corner far from the baseline grid. 


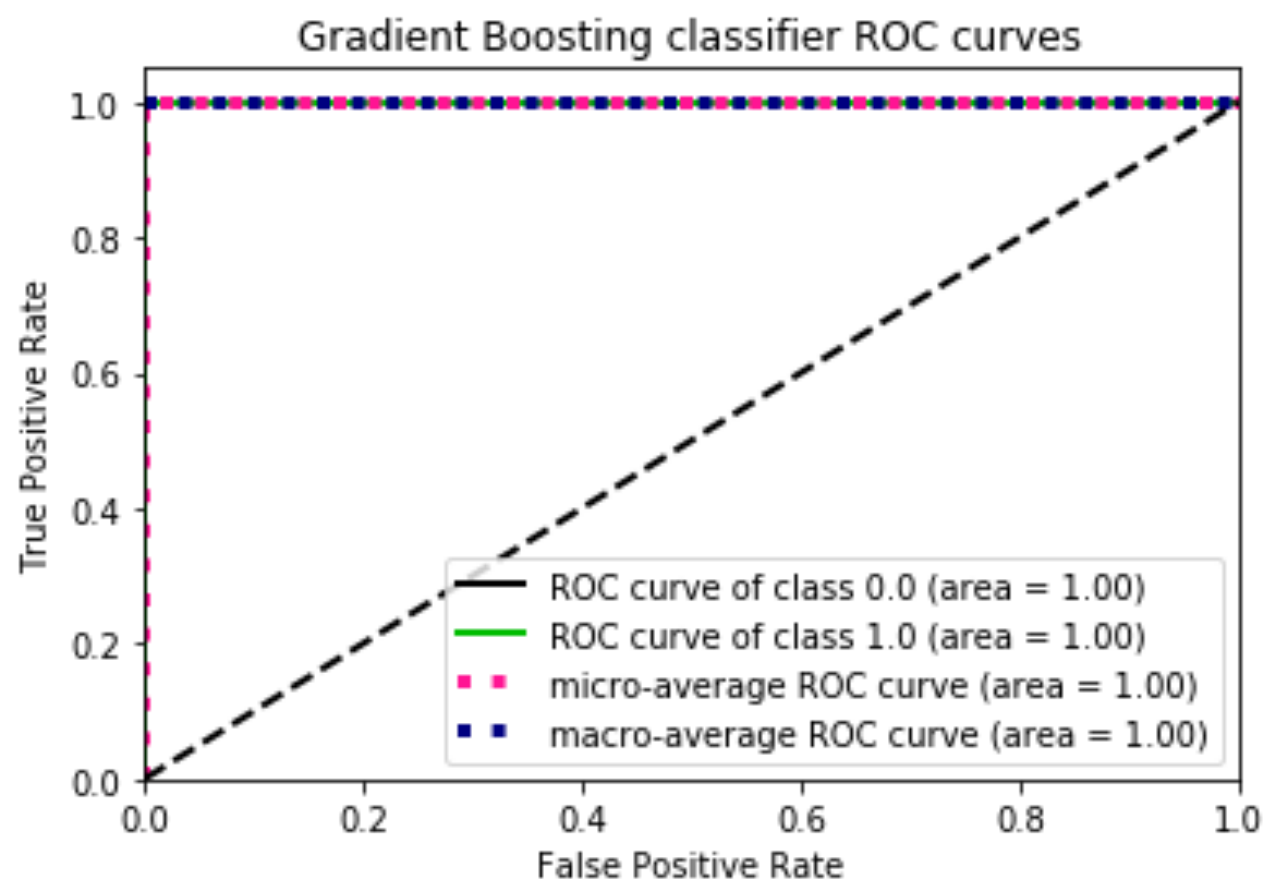

Figure 74: ROC curve obtained through ensemble Gradient Boosting classifier model

The aforementioned Figure 74 represents the ROC curve obtained through ensemble Gradient Boosting classification model; the model achieved an AUC of $100 \%$. The ROC curve does not interact with the baseline grid (red dashed lines) and it's perfectly situated towards the top left corner. 


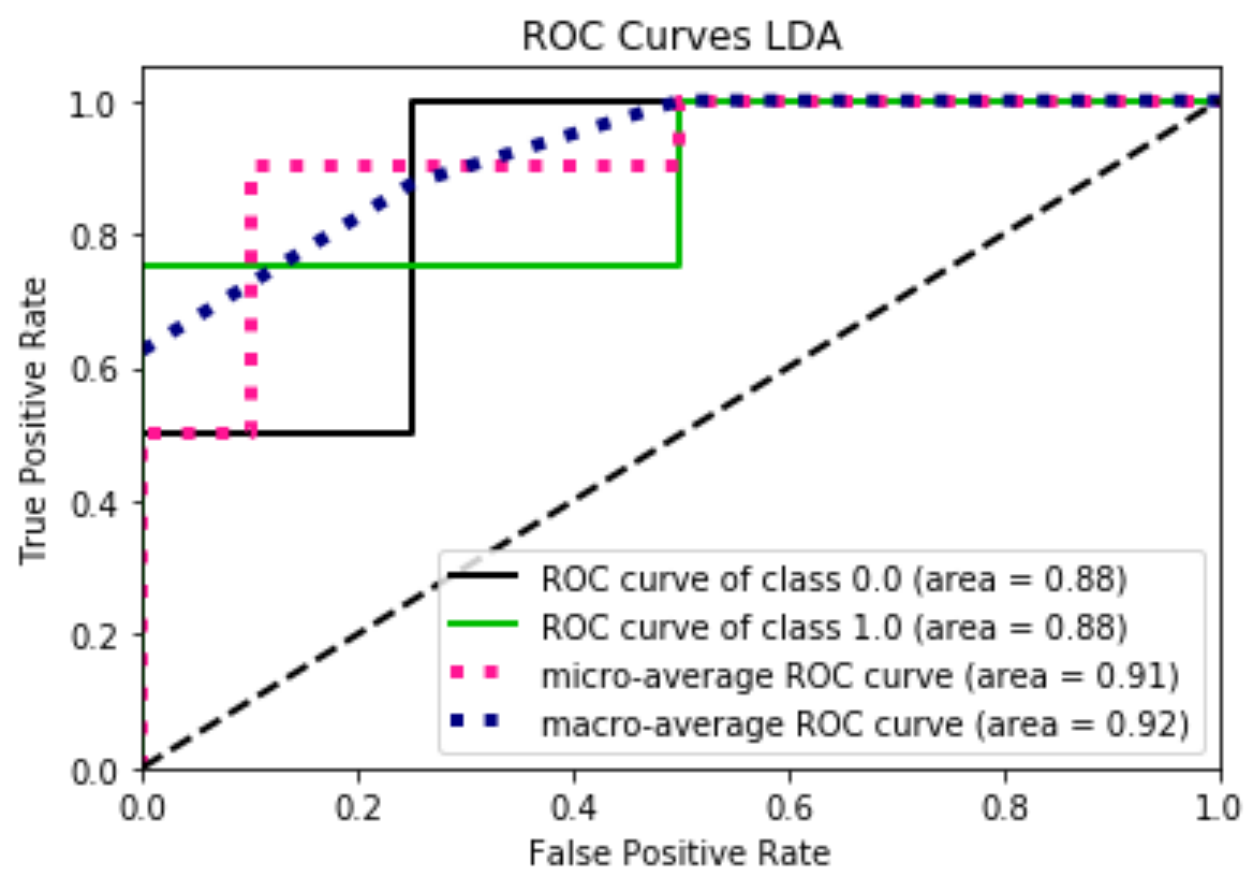

Figure 75: ROC curve obtained through LDA

The previous Figure 75 displays the ROC curve produced from LDA classification model, the model resulted in an AUC score of $0.88 \%$. 


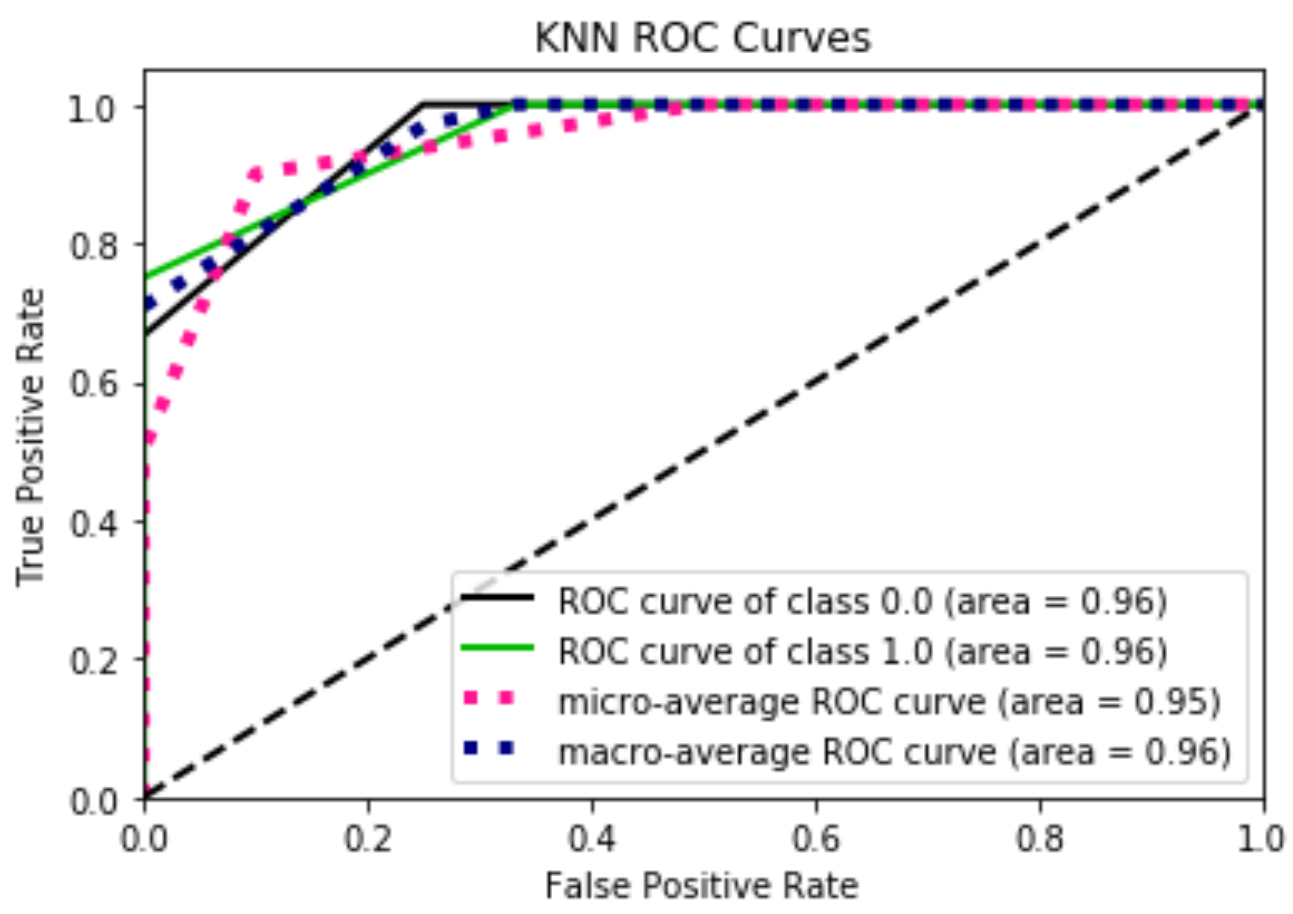

Figure 76: ROC curve depicted from KNN model

The Figure 76 above represents the ROC curve generated due to the performance of KNN classification model concerning the binary classification of stress and normal subjects. The model emanated an AUC score of $96 \%$. 


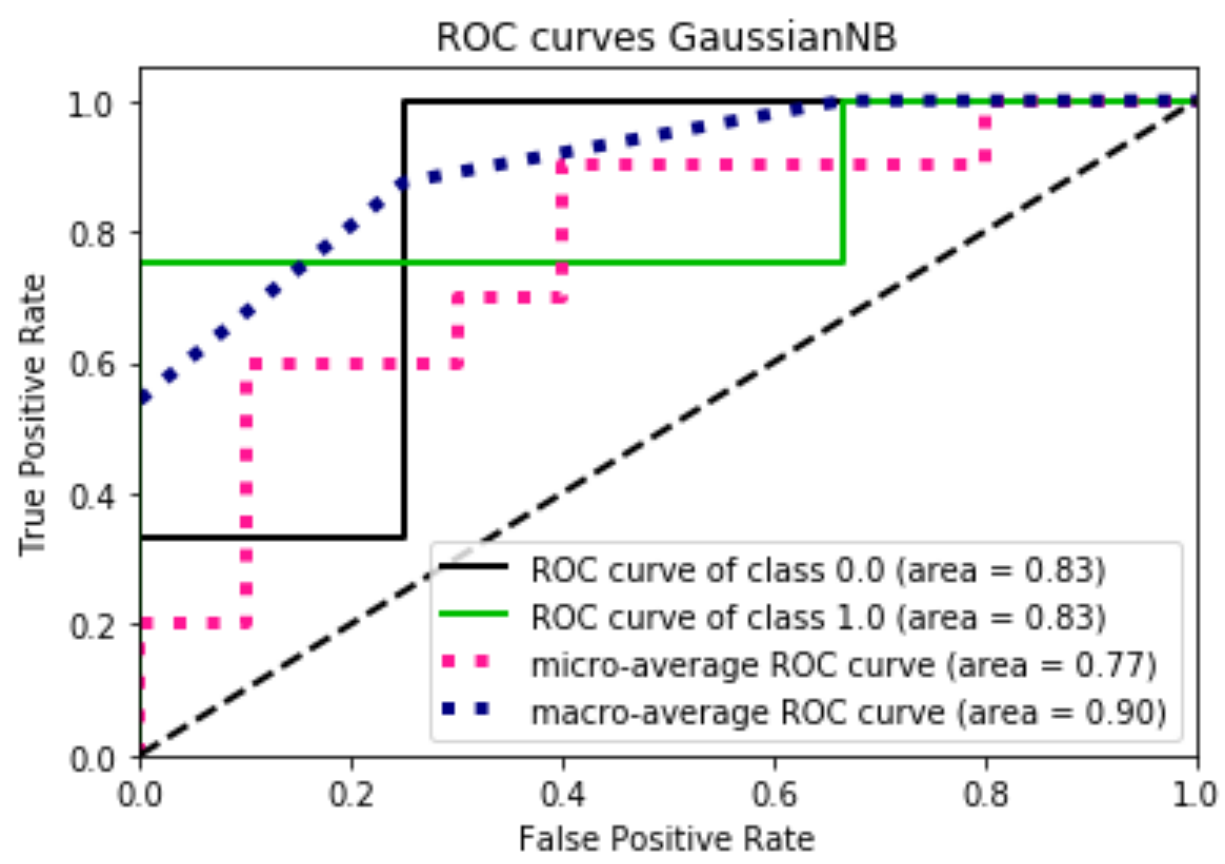

Figure 77: ROC curve produced from Naive Bayes model

The previous Figure 77 evince the ROC curve associated with binary classification of stress and normal subjects through Naive Bayes classification model. The model yielded an AUC score of $83 \%$. The ROC curve did not interact with the baseline grid.

\subsubsection{5-fold Cross Validation}




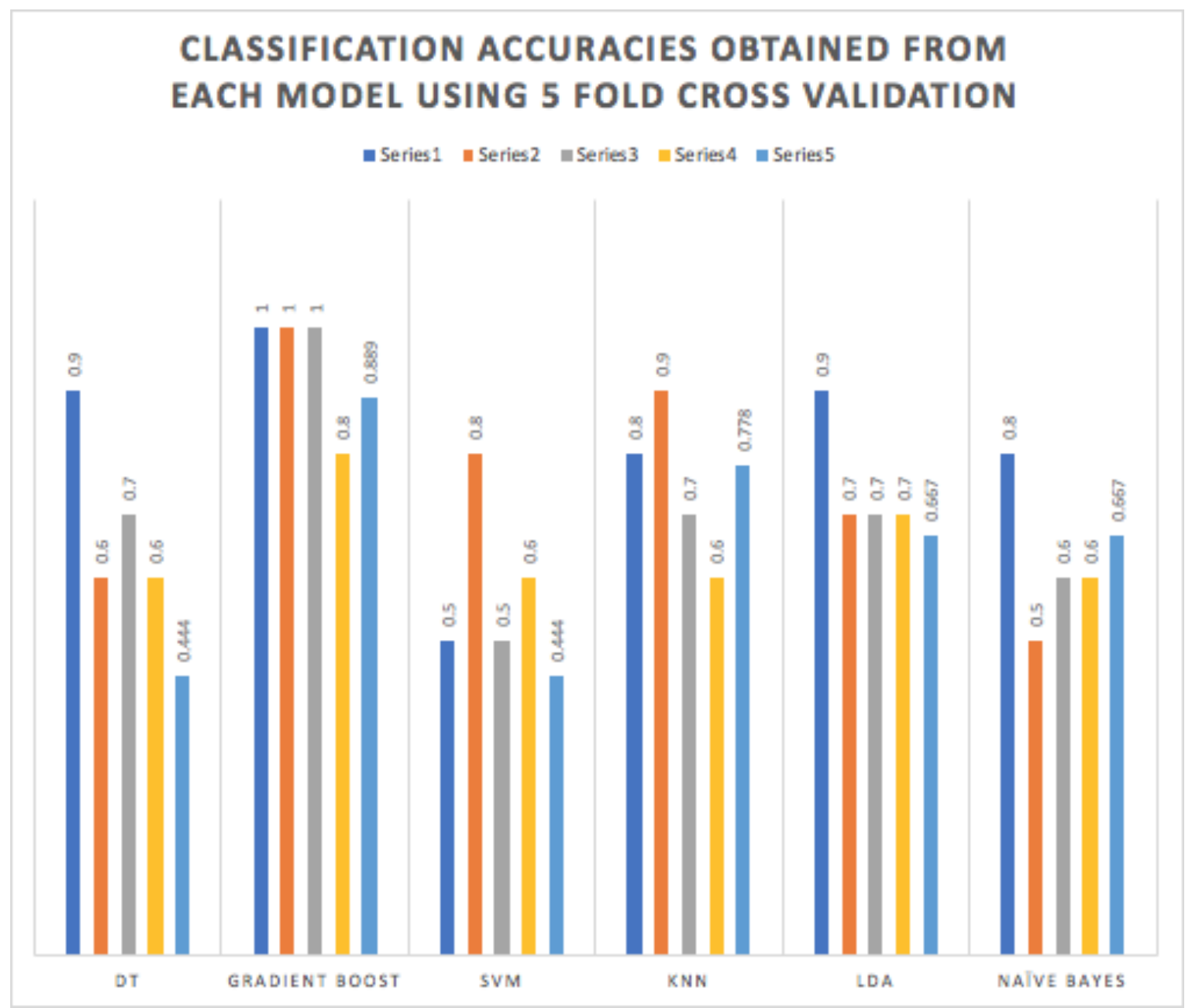

Figure 78: Classification accuracies obtained by each model following 5-Fold Cross Validation

Figure 78 illustrates the accuracy achieved by each model after splitting the dataset into 5 folds. This chart represents a more efficient analysis of the accuracies obtained and utilizes the small dataset more effectively. It yields the classification accuracy through various training and testing data splits. 


\section{Mean Classification Accuracy obtained from each model after 5-Fold CV}

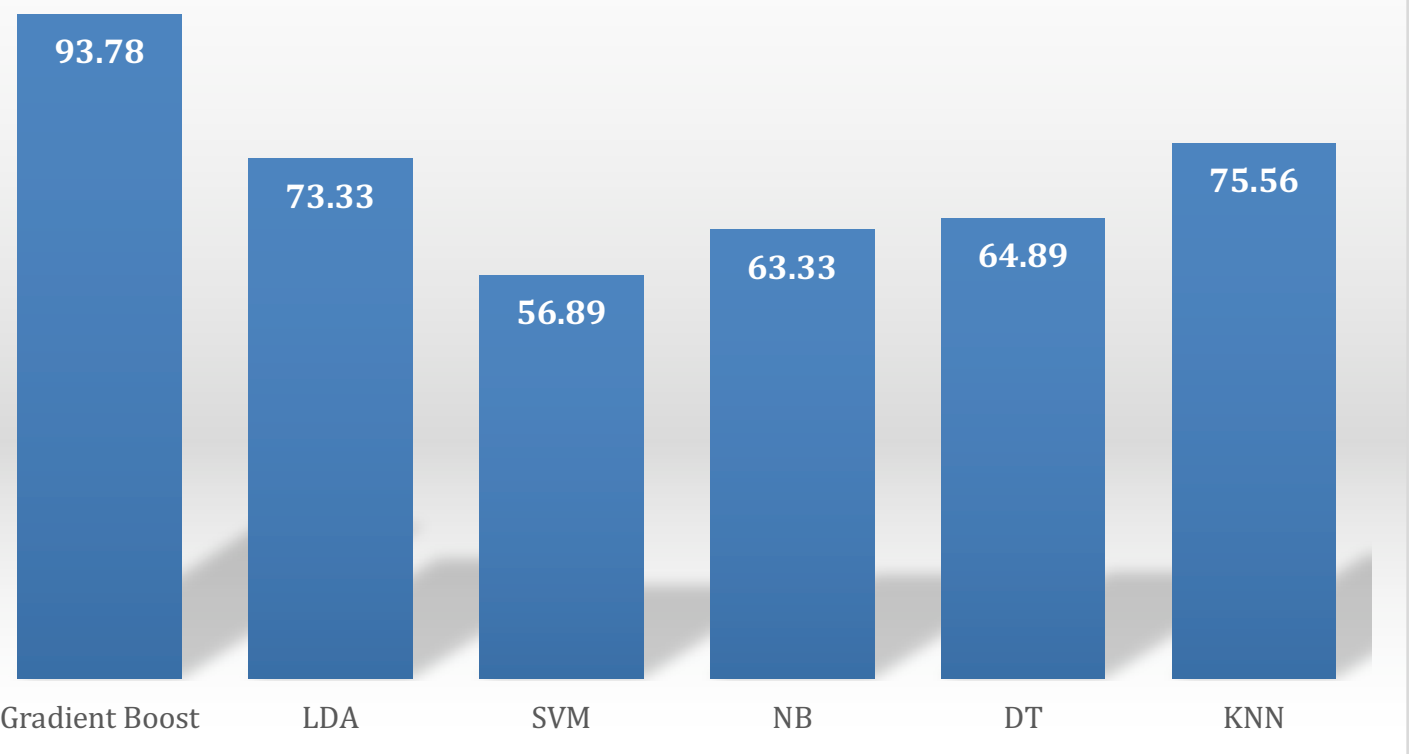

Figure 79: Mean Accuracies obtained by each model following 5-Fold Cross Validation

Figure 79 delineates the mean accuracies obtained from each model after 5-Fold cross validation, the results are a more accurate representation of the effectiveness of each model. 


\section{Standard Deviation of classification results obtained from each model after 5 -fold CV}

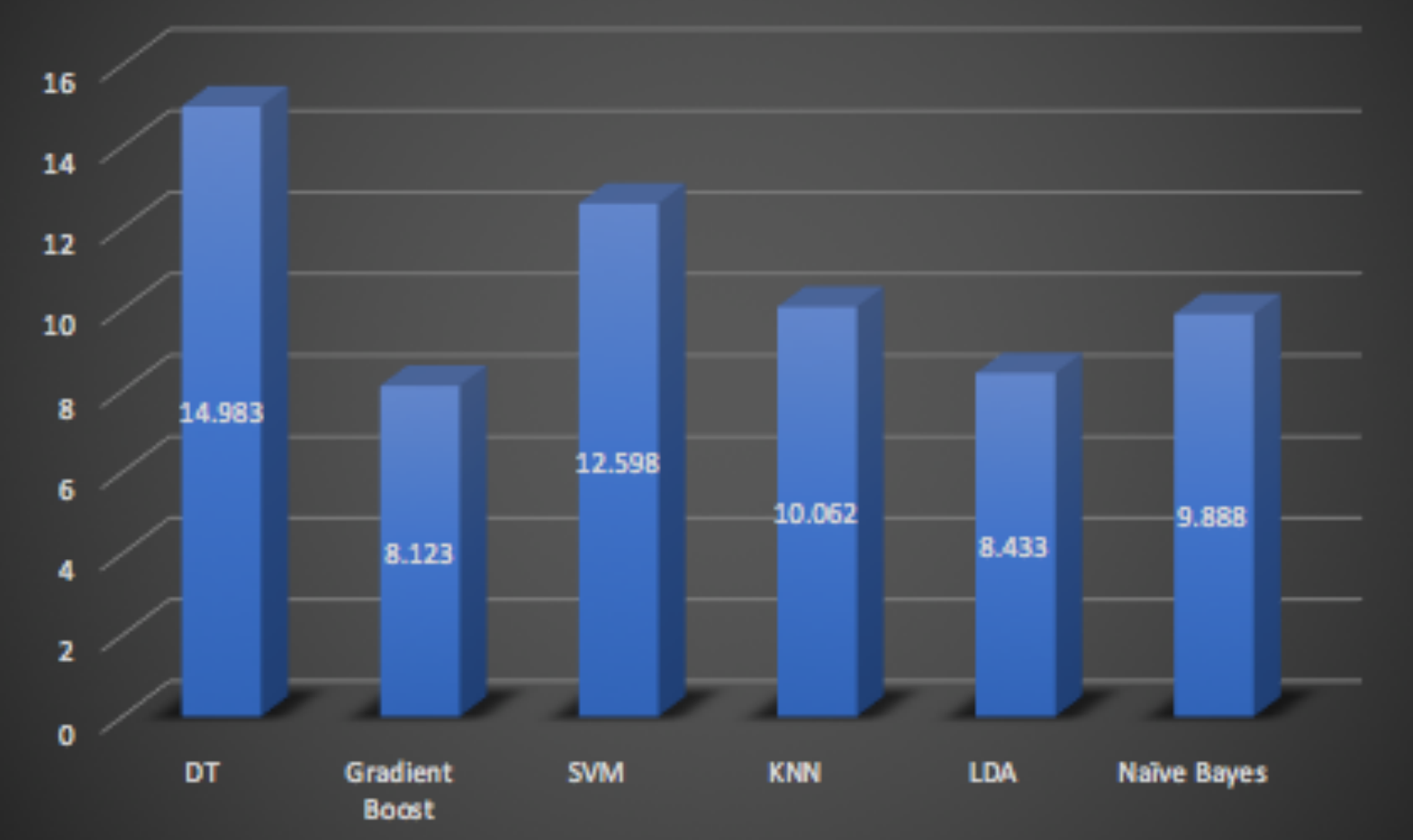

Figure 80: Standard Deviation obtained from each model following 5-Fold Cross Validation

Figure 80 depicts the standard deviation of the results obtained from each model, it effectively illustrates the distribution of the results based on different training and testing data splits. 


\subsection{Discussion}

The results from the correlation matrix further substantiate the proportional relationship between $L F, L F / H F$ ratio and stress, as well as the inverse proportional relationship between $H F, p N N 50$ and stress. There were a few subjects who produced contradictory results, instead of being stressed, they were rather more awake after the VR roller simulation, which is associated with low $L F$ and low $H F$. This would demonstrate that VR roller coaster may reduce $H F$, which ideally was meant to increase stress. Seemingly, it can also have a positive impact on a subject who is drowsy, by decreasing $H F$ and keeping low $L F$ stable, resulted in a subject who is more awake. The varying results signify the effects of affective emotion (such as fear and happiness) and perception on inducing different types of stress, (Abdi et al, 2018) revealed that stress can be a positive factor which increases our level of energy and focus [59]. Based on affective emotion and their perception associated with certain events, some subjects feel more awake while other subject's GSR level increases, thus resulting in an increase in skin temperature, sweating and stress. Stress can be negative factor which keeps subjects awake during the night time due to reduced $H F$ (drowsiness is associated with increased $H F$ and reduced $L F$ ) and increased $L F$, but if it is induced during the morning within a drowsy subject, it can result in being more awake due to the decrease in $H F$ and increased $L F$. The correlation matrix provides the correlation which justify that stress results in decreased $H F$, which would result in reduced drowsiness (drowsiness is associated with high $H F$ and low $L F$ ). The aforementioned features were used to develop various machine learning models which were used to determine the accuracy of detecting stress from the feature values. 

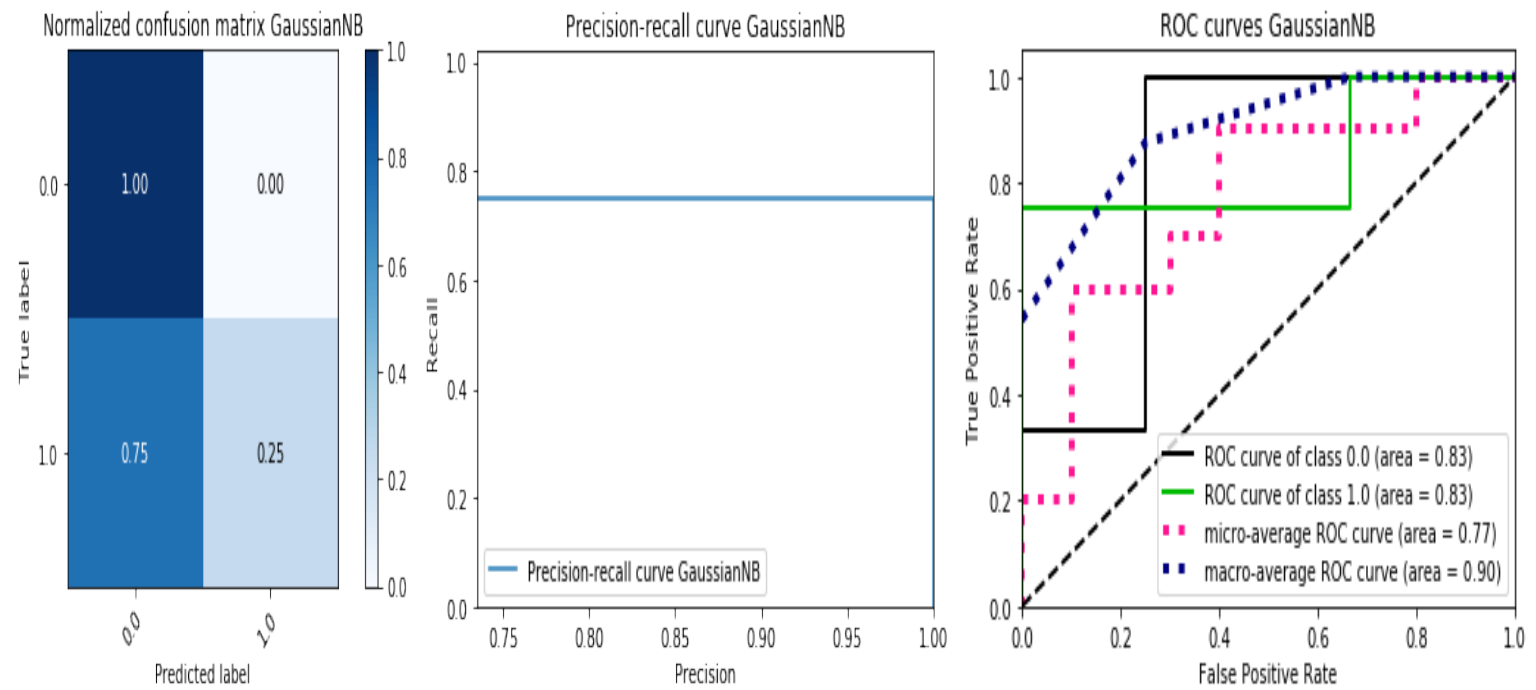

Figure 81: Evaluation of the performance of Naive Bayes model

The Naive Bayes model resulted in a poor classification accuracy of $70 \%$, it produced a TP rate of $25 \%$ and had an AUC score of $83 \%$. The results evidently make it apparent that the model performed inadequately, especially when it concerned stress detection. An AUC score of 83\% indicates that the model is capable of distinguishing between stress and relaxed subjects with $83 \%$ accuracy through the extracted features, which is alright by most standards but an AUC score of 0.5 indicates that the model not as effective other models for discriminating between stress and relaxation, this comparison illustrates that the model performed poorly and failed to efficiently detect stress.

The precision and recall curve are another method to evaluate the performance of Naive Bayes classifier which identifies the positive predictive value and sensitivity associated with stress detection. Detection of stress is broken down into four components which are TP, TN, FP and $F N$. TP indicates whether the label predicted was stress, $T N$ indicates whether the label predicted was relaxed, $F N$ indicates that stress was not detected, $F P$ indicates that stress was detected when the subject was not stressed. The Naive Bayes model produced a maximum sensitivity/recall of $25 \%$, which established that it was mediocre model to identify stress induced within each subject, out of every 10 subjects, this model would correctly identify 2 or 3 who were actually stressed through the features utilized. Precision/positive predictive value reveals 
whether the labels used to indicate stress are actually correct or not, are the subjects actually stressed due to VR roller coaster simulation or the cognitive color stroop task. The results from the precision recall curve indicates that at the range of $85 \%-100 \%$ precision, the recall is $75 \%$, this illustrates that the model is capable of correctly detecting $75 \%$ of stressed subject with at least $85 \%$ accuracy, demonstrating the effectiveness of the model.

Naive Bayes is a probabilistic classifier based on Bayes theorem, it's a fairly simple classifier which excels in classifying/analyzing sentiments from texts, documents which may relate to spam filters, sports or politics, recommendation systems which are used to predict ratings and preferences [95]. Naive Bayes performs effective classification with only independent labels and a small training dataset, the performance of the model is quite inadequate if a dependent label is utilized to predict the outcome from certain features which were used to describe an event. The binary labels used to indicate stress/relaxation had a high correlation with the HRV features, the labels were dependent on the variations and the values associated with the HRV features, the Naive Bayes model was not able to understand that behaviour, it simply predicted stress based on the pattern of the label alone because it assumes that the label is independent from the remaining features. The desideratum for an independent label is the root cause behind the low accuracy of classifying stress which is dependent on the variations associated with HRV features.
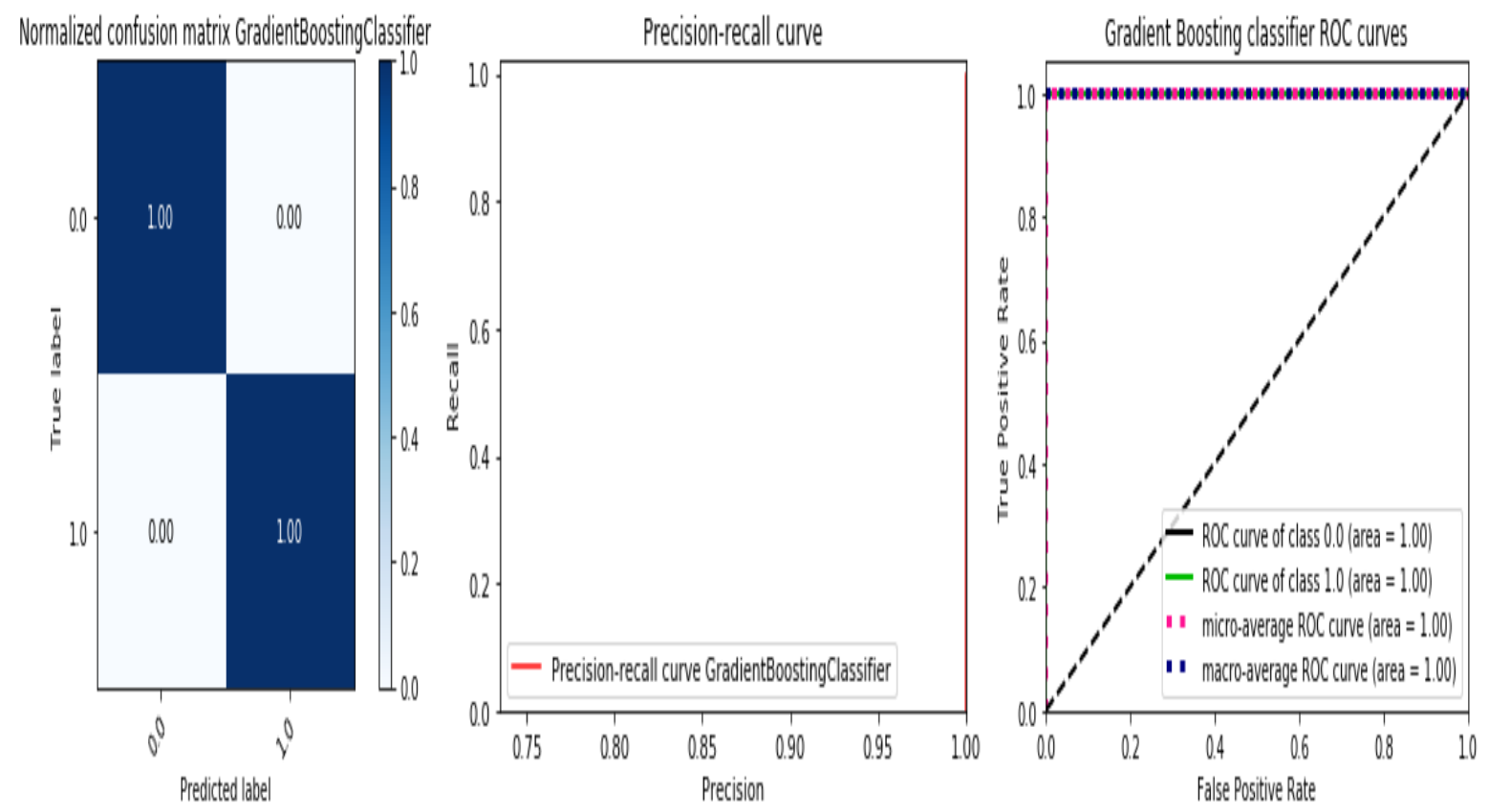
Figure 82: Evaluation of the performance of ensemble Gradient Boosting Classifier model

Ensemble Gradient Boosting Classifier classified binary labels of stress with 100\% accuracy. The model resulted in a precision and recall of 100\%. The ROC curve produced an AUC score of 1.00 which is also a perfect result. The results indicate that Gradient Boosting Classifier had a TP rate of $100 \%$, the model was capable of predicting stress with $100 \%$ accuracy based on the corresponding feature values and variation. The model had a $T N$ rate of $100 \%$, which reveals that it was also able to correctly identify events which led to a more relaxed/calmer subject very efficiently. The model produced a sensitivity and recall score of $100 \%$, this reveals that the model correctly identified every subject who were stressed due to certain phases, the aforementioned result also imparts that it was able to identify every phase which induced stress within each subject. The precision of the model was $100 \%$, this indicates that the model was able to correctly predict stress due to the corresponding features, similar values and variation associated with the features were correctly identified with the complementary label. The model was able to classify stressed subjects with $100 \%$ accuracy.

Ensemble Gradient Boost Classifier model is a strong learning tool, which is an efficient model for classification and regression analysis, similar to Decision Tree model. It converts weak learners (such as a Decision Tree algorithm) into strong learners. Weak learners are algorithms which aren't capable of adapting to a certain dataset, the classification accuracy achieved using such an algorithm is considered to be slightly above random. Computationally they tend to be very simple. Regression analysis identifies the strength of the relationship concerning the features and the corresponding label. Decision tree breaks down the process of classification into multiple preceding decision trees, each tree makes a decision based on certain outcomes or information that leads to another tree, a series of decisions are made which identifies the label as stressed or relaxed. Gradient Boost model is a modified version which improves upon the accuracy of predicting stress through each iteration after the first tree. It's an efficient model due to its ability to progressively train each model iteratively and sequentially. The model utilizes 
gradients within the loss function which enables the model to identify the errors associated with regression models, indicate the performance of the model when it is utilized for classifying stress. The model is prone to overfitting and more time consuming in comparison to other learners. The model was able to understand the relationship between stress and the corresponding features, make multiple decisions based on why certain values would result in a subject being stressed and why certain circumstances would lead to a subject being more relaxed based on the features presented.

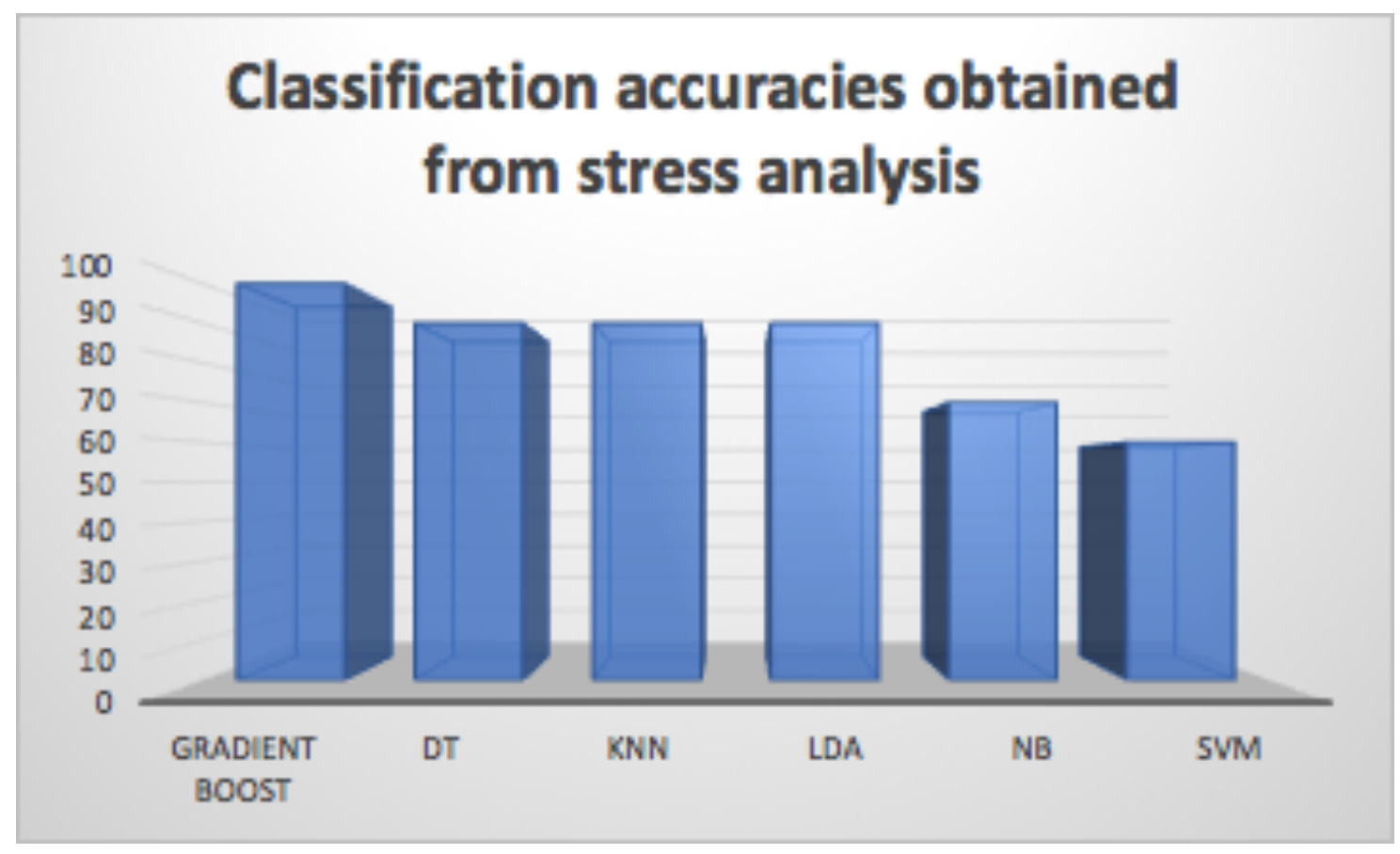

Figure 83: 3D column chart which displays the stress classification accuracy obtained using various machine learning models

The preceding Figure indicates the accuracy obtained through an array of classifiers. Ensemble Gradient Boosting classifier and NB was previously analyzed to reveal why NB performed poorly in comparison to ensemble Gradient Boosting classifier. Each model is a mathematical representation which can be used to describe certain processes. SVM is another machine learning model much like DT which can be used for classification and regression, it is capable of extreme kernel transformations, which can efficiently discriminate between labels. It is an effective model for handling complex dataset, without requiring additional transformations manually. A 
hyperplane was used to discriminate between stressed and relaxed subjects. The model received an accuracy of $60 \%$, with a $T P$ of $0 \%$ and $F N$ of $100 \%$. The model was not able to detect stressed subjects $100 \%$ of the time, out of every ten subjects, it won't be able to indicate a person who was stressed. When classifying stress, it's an inadequate model which is quite ineffective and although it can automatically transform complex data, it is also quite difficult to fine tune the parameters of SVM, it is also sensitive to overfitting making it hard to improve the efficiency of the model. It is also quite time consuming in comparison to other models. Unlike decision tree, the model is not efficient when it is utilized to interpret noisy data, which may explain why decision tree classifies with a higher accuracy than SVM, the inability to comprehend noisy data also reveals that the data is embedded with some noise.

The LDA model used to classify binary class associated with stress achieved an accuracy of $90 \%$. It performed on par with DT, it was able to detect stress with $75 \%$ accuracy as specified through the TP rate. The model resulted in a $F N$ rate of $25 \%$. The model performs ineffectively if the data drifts from normal distribution, resulting in consequential data which is non-gaussian and resulted in poor classification performance. The data utilized for stress classification was normally distributed as demonstrated through the p-value obtained using statistical t-test, except for few feature values, which were abnormally high or low due to certain instances and due to the noise associated with the signal. The LDA model unlike DT did not adapt well to the noise associated with the data and the slight non gaussian distribution.

KNN model similarly to DT model was able to classify stress and relaxed events with $90 \%$ accuracy, it's a sturdy model which can interpret complex data, it is easy to comprehend and implement. It was able to classify labels with high accuracy due to its ability to adapt to the dynamics of the data, much like human memory, it constantly interprets new data and habituates quickly to the changes associated. It can also be used for classification and regression analysis. It's a perfect model for data which includes less features for binary classification of stress/relax incidents, too many features increase the rate of $F N$ and results in missed classification/predictions. The resulting high accuracy exemplifies the balance of stress/relax occurrences due to each phase of the experiment; imbalanced labels often results in increased misclassification of the minority class. The dataset was fairly small, KNN works efficiently with 
smaller datasets, KNN is prone to overfitting when concerning large datasets. The model resulted in a $T P$ of $75 \%$ and $F N$ of $25 \%$, relaxed label was greater in comparison to stress class which would verify the reason behind the $25 \%$ missed classification of stress. A larger dataset might have been able to further balance the labels, which would most likely result in improved efficiency. One of the disadvantages of a small dataset is that it's hard to comprehend the accuracy of the $T P$ and $T N$ rate, a larger dataset would make the model more robust and definite with regards to stress classification.

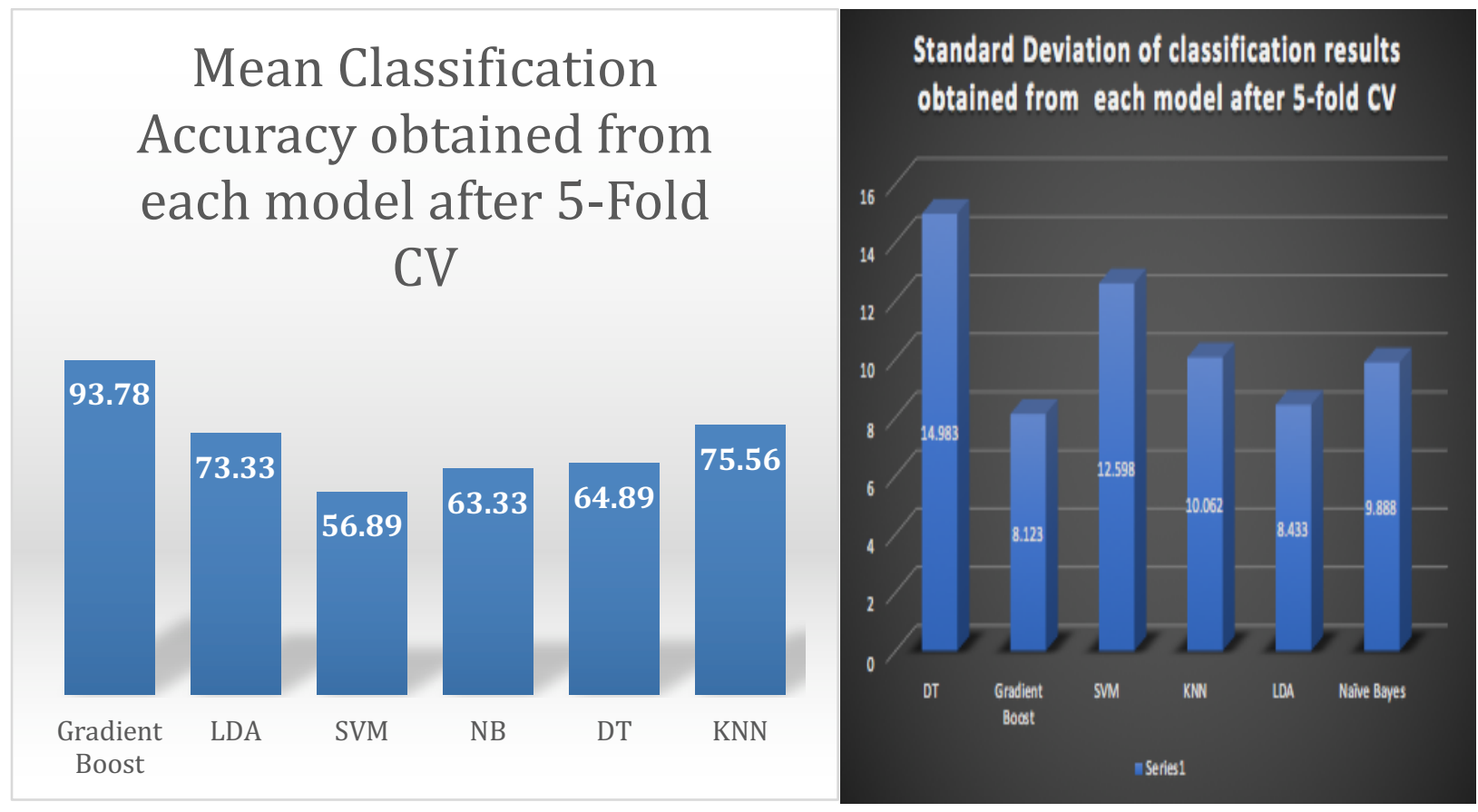

Figure 84: Mean accuracy and standard deviation obtained from 5-fold Cross Validation.

Cross validation is often utilized to prevent overfitting. Overfitting is a common problem associated with a small dataset. Figure 78 displayed previously in the results section revealed that although each model achieved high classification accuracy (excluding Naive bayes), the model may only perform well on this dataset and the classification results were not a good indication of model performance on other dataset. Figure 78 also enabled the identification of training and testing splits which led to the highest classification accuracies. Series 1 led to the 
highest accuracies for most models, but most models except for Ensemble gradient boosting classifier performed less adequately, while other training and testing splits were utilized. Figure80 specified that Gradient boosting classifier also resulted in the least standard deviation, indicating that the classification accuracy achieved did not vary far from the mean accuracy and it is probably capable of classifying other datasets with similar accuracies. Gradient boosting also achieved the highest mean accuracy after 5-fold cross validation, which was $18 \%$ higher than KNN classifier, $20 \%$ greater than LDA and 28\% greater than DT classifier. This further emphasizes the effectiveness of the model and superiority over other competent classification models. From the results obtained after 5-Fold cross validation, it is noticeable from the rate of error obtained (SD from Figure 80) that SVM and DT models overfit the data. The high accuracy obtained significantly dropped and the models also produced a high variance. This could be prevented by tuning the regularization on DT and SVM. Gradient Boosting classifier is a strong learner, which used constraints on the amount of DT utilized in order to reduce error and prevent overfitting. 


\section{Chapter 5}

\section{Conclusion}

\subsection{Conclusion}

Heart rate variability represents the rate of variability associated with the variations in time intervals, with respect to each successive heartbeat. When you consider the difference between time intervals presented in milliseconds, a healthy and normal heart functions at an optimal rate when it is subjected to constant variation. The simplest method to derive HRV is through the analysis of an ECG signal, time and frequency domain analysis of the $R-R$ intervals obtained from a QRS complex is the most common approach to analyze human physiology, due to the rate of change associated with HRV. The sympathetic and parasympathetic nervous systems (SNS, PNS respectively) is ideally recognized as the branch of the autonomic nervous system (ANS) responsible for regulating HRV. Increased HRV results in an increase in PNS activity, which is associated with normal and healthy functions of the heart, leading to proper digestion of nutrients, relaxation during free time, reduced heart rate, greater capability to adapt to change in HRV, leading to faster recovery. Decreased HRV results in an increased activity of the sympathetic nervous system, which is often associated with various cardiac morbidity, mortality and increased stress. Some of the negative consequences and major diseases associated with reduced HRV are hypertension, myocardial infarction, diabetes, sleep apnea, cardiac burnout and chronic stress.

HRV is a very popular research subject, which has been analyzed to indicate the cause and effect of various pathologies, impact of SNS and PNS activity on athletic performance during a competition and as a result of overwork/performance burnout, $L F$ and $H F$ variations associated with drowsiness and car accidents, as well as other feature variations associated with acute and chronic stress, . Various medical devices such as an ECG, Respiration, GSR, PPG were utilized for signal acquisition, in order the extract essential features such as $L F, H F, L F / H F$ ratio, RMSSD, SDNN and ApEn subsequently after the preprocessing of the aforementioned signals. Numerous research paper applied machine learning algorithms to wirelessly classify various pathologies, acute and chronic stress, drowsiness through supervised machine learning methodologies and artificial neural network, antedating the astonishing future that can be 
developed from the advancement of artificial intelligence, concerning the remote telemonitoring of biomedical signals. This thesis paper acknowledged the impact of VR simulations and video games on stress levels through the wireless analysis of HRV and other physiological signals.

In order to obtain the physiological parameters required to analyze stress, 14 subjects were experimented on using 4 different trials, which included: 1) Baseline phase which consisted of doing nothing, 2) VR simulation was utilized to induce stress, anxiety and negative emotions, 3) Cognitive color stroop task was meant to cause anxiety and induce stress and 4) VR fishing game was the last phase of the experiment that was supposed to reduce stress and allow the subjects to relax. The signals were obtained wirelessly using ECG, GSR and Respiration sensors, their respective physiological features were extracted through MATLAB and python. Poincare plots were utilized for the first time in order to provide a non-linear analysis of stress from video games. Given that $A p E n$ shares a 0.811 correlation with $L F / H F$ ratio, it is seemingly a more effective method than other time domain methods to detect normal/abnormal HRV, observe beat to beat HRV and sympathovagal balance. The results indicated that perception played a major role with regards to stress, subjects who were afraid of roller coaster rides, a lack of interest/attention resulted in increased stress levels, increased sweating, increased respiration rate and increased anxiety, which was associated with distress. Subjects who enjoyed roller coasters, video games and cognitive tasks were energized, awake and relaxed throughout the process, as a result of eustress. All the significant results $(\mathrm{p}<0.05)$ from table 2 indicated that following VR fishing game, average $L F / H F$ (lomb) reduced from 1.2 to $0.928, V L F$ reduced from $2900\left(m s^{\wedge} 2\right)$ to $2120\left(m s^{\wedge} 2\right), L F$ reduced from $35472.50\left(m s^{\wedge} 2\right)$ to $4963.44\left(m s^{\wedge} 2\right)$, GSR_mean reduced from 5.665 to 3.257 , respiration was normal 24.3454 breaths/min and $H R$ reduced from 103.52 to 92.5 beats/min. The results are a testament to the positive impact produced from the VR fishing game, physiological signal analysis revealed that VR fishing game reduced stress, improved HRV and decreased HR. It proved to be an entertaining and effective method to distract subject from their worries in a positive manner. The next step of the experiment involved the use of physiological features for automatic classification of stress, through machine learning algorithms aka artificial intelligence.

Machine learning is prevalent everywhere including finance, healthcare and biomedical research. LDA, SVM, KNN, DT, NB and ensemble gradient boosting classifier models were utilized to 
classify binary classes (stress, relax) from the HRV features extracted. Ensemble methods combine multiple machine learning algorithms in order to increase model performance, it is considered a strong learner. Gradient boosting ensemble method was able to classify labels with $100 \%$ accuracy, it is capable of improving model performance through progressive iteration and sequential training of the model. This was the first time EGB model was used to classify stress from a VR simulation and video game. Naive Bayes performed poorly, it was capable of classifying labels with $70 \%$ accuracy and resulted in an inadequate $T P$ rate of $25 \%$, it was an ineffective model to classify stress. There were several limitations which could be the potential reason behind mediocre performance, illogical feature values and disagreeable p-value.

\subsection{Limitations}

There were several limitations associated with the study conducted which led to irrational feature values, noisy signals, p-value above 0.05 and poor classification accuracy. The ECG sensor had direct skin contact which made it possible to retrieve accurate signals, but the signals were susceptible to intrinsic body noise, environmental noise and electrode interference noise. Baseline wander are low frequency artifacts (error associated with the signal) associated with most ECG signals. Increased sweat activity can cause loose contact between the subjects and the sensors, which leads to increased power line interference. Although filters were applied to remove the noise associated with the signal, in order to retain most of the valuable information, the filters were utilized with caution, leading to some erroneous results. The dataset utilized for machine learning classification was pretty small, a larger dataset may increase the classification accuracy and TP rate, obtained through the models. Movement should be better maintained in order to reduce motion artifacts. EMD is another option to reduce baseline wander but whether it performs better than a notch filter remains a question. Subjects were not trained on the subject, so they were unaware of the effect of their actions and movements, using researchers as the subjects might lead to the acquisition of more efficient signals. The controllers for the fish game required a lot of hand movement and it made contact with the GSR sensors, a different game which requires vocal control might lead to more efficient results. The data was labelled manually by me based on input feature values/variation which makes the data a bit biased. A larger dataset which include labelled activities would be more optimal. It would also prevent DT and SVM from overfitting the data. The reduction of errors is only one part of the future work. 


\subsection{Future Work}

There are a lot of room for further research and experiments, it involves a more in-depth analysis of stress. Regular subjects were assessed for this research study, but for the future, the plan is to make it more specific, concerning particular working professionals and individuals, with respect to their health. Nurses suffer from work related stress due to irregular working hours; it is a possible research study in the future. The epoch utilized for this study was $5-8$ minutes, which does not correlate to real-time, real-time analysis of stress using 30 second epochs is also a goal for the future. Smartphones are currently a popular device to remotely analyze HRV, it can be utilized to classify stress through cloud computing. This study classified stress from binary classes, currently the goal for the near future involves classifying 5 labels of stress. Stress was classified using data obtained solely from ECG signals, data fusion which integrates multiple data sources can produce information, which is more coherent, accurate and pragmatic. A more in-depth statistical analysis can further reduce bias, comprehend the data more effectively and draw better conclusions. Creating an app to analyze stress, few years from now. Heart rate variability has a wide variety of applications, research in this domain has an immense potential for growth, especially with the rise of medical devices, biomedical wristbands and watches. Iphones and smartphones are incorporating apps which allows for remote analysis of HRV, through data obtained from the aforementioned devices, continued effort and progress can cause an enormous impact within the lives of patients suffering from cardiovascular diseases, which could allow them to perform their daily activities, travel and report the status of their health to their physician from anywhere. Telemonitoring of patients is far from perfect but continued progress in innovation and research can help millions of people suffering from diseases around the world. This will provide $24 \mathrm{~h}$ medical surveillance, continuous feedback and also allow the patients to live their usual life, instead of being restricted to a bed for regular check-ups. 


\section{References}

[1] Boundless. (n.d.). Boundless Anatomy and Physiology. Retrieved from https://courses.lumenlearning.com/boundless-ap/chapter/functions-of-the-autonomic-nervoussystem/

[2] Rosenberg, W. V., Chanwimalueang, T., Adjei, T., Jaffer, U., Goverdovsky, V., \& Mandic, D. P. (2017). Resolving Ambiguities in the $L F / H F$ Ratio: $L F-H F$ Scatter Plots for the Categorization of Mental and Physical Stress from HRV. Frontiers in Physiology, 8. doi:10.3389/fphys.2017.00360

[3] Vicente, J., Laguna, P., Bartra, A., \& Bailón, R. (2016). Drowsiness detection using heart rate variability. Medical \& Biological Engineering \& Computing, 54(6), 927-937. doi:10.1007/s11517-015-1448-7

[4] Georgiou, K., Larentzakis, A. V., Khamis, N. N., Alsuhaibani, G. I., Alaska, Y. A., \& Giallafos, E. J. (2018). Can Wearable Devices Accurately Measure Heart Rate Variability? A Systematic Review. Folia Medica, 60(1). doi:10.2478/folmed-2018-0012

[5] Landolt, K., Maruff, P., Horan, B., Kingsley, M., Kinsella, G., O’Halloran, P. D., .. . Wright, B. J. (2017). Chronic work stress and decreased vagal tone impairs decision making and reaction time in jockeys. Psychoneuroendocrinology, 84, 151-158. doi:10.1016/j.psyneuen.2017.07.238 [6] Rodrigues, S., Paiva, J., Dias, D., Aleixo, M., Filipe, R., \& Cunha, J. (2018). Cognitive Impact and Psychophysiological Effects of Stress Using a Biomonitoring Platform. International Journal of Environmental Research and Public Health, 15(6), 1080. doi:10.3390/ijerph15061080

[7] Walker, E. D., Brammer, A., Cherniack, M. G., Laden, F., \& Cavallari, J. M. (2016). Cardiovascular and stress responses to short-term noise exposures-A panel study in healthy males. Environmental Research, 150, 391-397. doi:10.1016/j.envres.2016.06.016

[8] Blood, J. D., Wu, J., Chaplin, T. M., Hommer, R., Vazquez, L., Rutherford, H. J., . . . Crowley, M. J. (2015). The variable heart: High frequency and very low frequency correlates of depressive symptoms in children and adolescents. Journal of Affective Disorders, 186, 119-126. doi:10.1016/j.jad.2015.06.057

[9] Shiro, Y., Arai, Y. P., Matsubara, T., Isogai, S., \& Ushida, T. (2012). Effect of muscle load tasks with maximal isometric contractions on oxygenation of the trapezius muscle and sympathetic nervous activity in females with chronic neck and shoulder pain. BMC Musculoskeletal Disorders, 13(1). doi:10.1186/1471-2474-13-146

[10] Leti, T., \& Bricout, V. A. (2013). Interest of analyses of heart rate variability in the prevention of fatigue states in senior runners. Autonomic Neuroscience, 173(1-2), 14-21. doi:10.1016/j.autneu.2012.10.007 
[11] Lu, D., Yang, A. C., Cheng, H., Lu, T., Yu, W., Chen, C., \& Sung, S. (2016). Heart Rate Variability Is Associated with Exercise Capacity in Patients with Cardiac Syndrome X. Plos One, 11(1). doi:10.1371/journal.pone.0144935

[12] Walker, E. D., Brammer, A., Cherniack, M. G., Laden, F., \& Cavallari, J. M. (2016). Cardiovascular and stress responses to short-term noise exposures - A panel study in healthy males. Environmental Research, 150, 391-397. doi:10.1016/j.envres.2016.06.016

[13] Wang, Y., Wei, S., Zhang, S., Zhang, Y., Zhao, L., Liu, C., \& Murray, A. (2018). Comparison of time-domain, frequency-domain and non-linear analysis for distinguishing congestive heart failure patients from normal sinus rhythm subjects. Biomedical Signal Processing and Control, 42, 30-36. doi:10.1016/j.bspc.2018.01.001

[14] Pinheiro, A. D., Pereira, V. L., Baltatu, O. C., \& Campos, L. A. (2015). Cardiac autonomic dysfunction in elderly women with myocardial infarction. Current Medical Research and Opinion,31(10), 1849-1854. doi:10.1185/03007995.2015.1074065

[15] Toni, G., Murri, M. B., Piepoli, M., Zanetidou, S., Cabassi, A., Squatrito, S., . . Amore, M. (2016). Physical Exercise for Late-Life Depression: Effects on Heart Rate Variability. The American Journal of Geriatric Psychiatry, 24(11), 989-997. doi:10.1016/j.jagp.2016.08.005

[16] Shi, H., Yang, L., Zhao, L., Su, Z., Mao, X., Zhang, L., \& Liu, C. (2017). Differences of Heart Rate Variability Between Happiness and Sadness Emotion States: A Pilot Study. Journal of Medical and Biological Engineering, 37(4), 527-539. doi:10.1007/s40846-017-0238-0

[17] Ponnusamy, A., Marques, J. L., \& Reuber, M. (2012). Comparison of heart rate variability parameters during complex partial seizures and psychogenic nonepileptic seizures. Epilepsia, 53(8),1314-1321. doi:10.1111/j.1528-1167.2012.03518.x

[18] Abad, C., Kobal, R., Kitamura, K., Gil, S., Pereira, L., Loturco, I., \& Nakamura, F. (2015). Heart rate variability in elite sprinters: Effects of gender and body position. Clinical Physiology and Functional Imaging, 37(4), 442-447. doi:10.1111/cpf.12331

[19] Yoshizaki, T., Midorikawa, T., Hasegawa, K., Mitani, T., Komatsu, T., \& Togo, F. (2014). Associations between Diurnal 24-Hour Rhythm in Ambulatory Heart Rate Variability and the Timing and Amount of Meals during the Day Shift in Rotating Shift Workers. PLoS ONE, 9(9). doi:10.1371/journal.pone.0106643

[20] Koenig, J., Jarczok, M. N., Ellis, R. J., Warth, M., Hillecke, T. K., \& Thayer, J. F. (2014). Lowered Parasympathetic Activity in Apparently Healthy Subjects with Se $L F$-Reported Symptoms of Pain: Preliminary Results from a Pilot Study. Pain Practice, 15(4), 314-318. doi:10.1111/papr.12177

[21] Park, C., Lee, S., Park, H., Baik, Y., Park, Y., \& Park, Y. (2010). Autonomic function, voice, and mood states. Clinical Autonomic Research, 21(2), 103-110. doi:10.1007/s10286-0100095-1 
[22] Choudhary, A. K., Alam, T., Dhanvijay, A. K., \& Kishanrao, S. S. (2018). Sleep restriction progress to cardiac autonomic imbalance. Alexandria Journal of Medicine, 54(2), 149-153. doi:10.1016/j.ajme.2017.05.003

[23] Huang, J., Chen, C., Chang, C., Chen, S., Hsieh, M., Hsieh, Y., \& Chen, H. (2017). Effects of stroke on changes in heart rate variability during hemodialysis. BMC Nephrology, 18(1). doi:10.1186/s12882-017-0502-0

[24] Chanwimalueang, T., Aufegger, L., Rosenberg, W. V., \& Mandic, D. P. (2016). Modelling stress in public speaking: Evolution of stress levels during conference presentations. 2016 IEEE International Conference on Acoustics, Speech and Signal Processing (ICASSP). doi:10.1109/icassp.2016.7471788

[25] Hallman, D. M., Holtermann, A., Søgaard, K., Krustrup, P., Kristiansen, J., \& Korshøj, M. (2017). Effect of an aerobic exercise intervention on cardiac autonomic regulation: A worksite RCT among cleaners. Physiology \& Behavior, 169, 90-97. doi:10.1016/j.physbeh.2016.11.031

[26] Hoogerwerf, M. D., Veldhuizen, I. J., Tarvainen, M. P., Merz, E., E. M. J. Huis In 'T Veld, W. L. A. M. De Kort, ... Frings-Dresen, M. H. (2018). Physiological stress response patterns during a blood donation. Vox Sanguinis, 113(4), 357-367. doi:10.1111/vox.12646

[27] Farooqi, M. E., \& Ali, I. (2018). Effects of Stress and Stress Hormones on Heart Rate Variability Parameters in 1st Year Medical Students. International Journal of Physiology, 6(2), 158. doi:10.5958/2320-608x.2018.00073.2

[28] Howells, F. M., Rauch, H. G., Ives-Deliperi, V. L., Horn, N. R., \& Stein, D. J. (2013). Mindfulness based cognitive therapy may improve emotional processing in bipolar disorder: Pilot ERP and HRV study. Metabolic Brain Disease, 29(2), 367-375. doi:10.1007/s11011-0139462-7

[29] Amaral, J. A., Guida, H. L., Abreu, L. C., Barnabé, V., Vanderlei, F. M., \& Valenti, V. E. (2016). Effects of auditory stimulation with music of different intensities on heart period. Journal of Traditional and Complementary Medicine, 6(1), 23-28. doi:10.1016/j.jtcme.2014.11.032

[30] Rios-Aguilar, S., Merino, J. L., Sánchez, A. M., \& Valdivieso, Á S. (2015). Variation of the Heartbeat and Activity as an Indicator of Drowsiness at the Wheel Using a Smartwatch. International Journal of Interactive Multimedia and Artificial Intelligence, 3(3), 96. doi:10.9781/ijimai.2015.3313

[31] Lee, J., Kim, J., \& Shin, M. (2017). Correlation Analysis between Electrocardiography (ECG) and Photoplethysmogram (PPG) Data for Driver's Drowsiness Detection Using Noise Replacement Method. Procedia Computer Science, 116, 421-426. doi:10.1016/j.procs.2017.10.083 
[32] Mohammed, A. A., Kheder, M. Q., \& Muhammed, A. W. (2015). Improved Driver Drowsiness Monitoring System using Real-time Eye Blinking Method. Journal of Zankoy Sulaimani - Part A,17(4), 9-18. doi:10.17656/jzs.10420

[33] Lee, J., Lee, S., Kim, C., Kim, K., \& Kwon, O. (2014). Detection of drowsy driving based on driving information. 2014 International Conference on Information and Communication Technology Convergence (ICTC). doi:10.1109/ictc.2014.6983224

[34] Sangeetha, M. (2015). Embedded ECG Based Real Time Monitoring and Control of Driver Drowsiness Condition. International Journal of Science, Technology and Society, 3(4), 176. doi:10.11648/j.ijsts.20150304.17

[35] Roy, R., \& Venkatasubramanian, K. (2015). EKG/ECG based Driver Alert System for Long Haul Drive. Indian Journal of Science and Technology, 8(19).

doi:10.17485/ijst/2015/v8i19/77014

[36] Jung, S., Shin, H., \& Chung, W. (2014). Driver fatigue and drowsiness monitoring system with embedded electrocardiogram sensor on steering wheel. IET Intelligent Transport Systems, 8(1), 43-50. doi:10.1049/iet-its.2012.0032

[37] Georgiou, K., Larentzakis, A. V., Khamis, N. N., Alsuhaibani, G. I., Alaska, Y. A., \& Giallafos, E. J. (2018). Can Wearable Devices Accurately Measure Heart Rate Variability? A Systematic Review. Folia Medica, 60(1). doi:10.2478/folmed-2018-0012

[38] Igasaki, T., Nagasawa, K., Murayama, N., \& Hu, Z. (2015). Drowsiness estimation under driving environment by heart rate variability and/or breathing rate variability with logistic regression analysis. 2015 8th International Conference on Biomedical Engineering and Informatics (BMEI). doi:10.1109/bmei.2015.7401498

[39] Rahim, H. A., Dalimi, A., \& Jaafar, H. (2015). Detecting Drowsy Driver Using Pulse Sensor. Jurnal Teknologi, 73(3). doi:10.11113/jt.v73.4238

[40] Tokutake, H., Sugimoto, Y., \& Shirakata, T. (2013). Real-time identification method of driver model with steering manipulation. Vehicle System Dynamics, 51(1), 109-121. doi:10.1080/00423114.2012.715175

[41] Bergmair, R. (2009). A proposal on evaluation measures for RTE. Proceedings of the 2009 Workshop on Applied Textual Inference - TextInfer 09. doi:10.3115/1708141.1708144

[42] Lenis, G., Reichensperger, P., Sommer, D., Heinze, C., Golz, M., \& Dössel, O. (2016). Detection of microsleep events in a car driving simulation study using electrocardiographic features. Current Directions in Biomedical Engineering, 2(1). doi:10.1515/cdbme-2016-0063

[43] Doudou, M., \& Bouabdallah, A. (2018). Performance Specifications of Market Physiological Sensors for Efficient Driver Drowsiness Detection System. Proceedings of the 7th International Conference on Sensor Networks. doi:10.5220/0006607800990106 
[44] Rogado, E., Garcia, J., Barea, R., Bergasa, L., \& Lopez, E. (2009). Driver fatigue detection system. 2008 IEEE International Conference on Robotics and Biomimetics.

doi:10.1109/robio.2009.4913155

[45] Purnamasari, P. D., \& Hazmi, A. Z. (2018). Heart Beat Based Drowsiness Detection System for Driver. 2018 International Seminar on Application for Technology of Information and Communication. doi:10.1109/isemantic.2018.854978

[46] Joseph, L. J. (2016). Arduino based real time driver drowsiness detection and mobile alert system using bluetooth. International Journal Of Engineering And Computer Science.

doi:10.18535/ijecs/v5i9.53

[47] Gontier, C. (2017). How to prevent mind-wandering during an EVA ? Presentation of a mind-wandering detection method using ECG technology in a Mars-analog environment. Acta Astronautica, 140, 105-112. doi:10.1016/j.actaastro.2017.08.008

[48] Sahayadhas, A., Sundaraj, K., \& Murugappan, M. (2013). Drowsiness detection during different times of day using multiple features. Australasian Physical \& Engineering Sciences in Medicine,36(2), 243-250. doi:10.1007/s13246-013-0200-6

[49] Virtanen, I., Kalleinen, N., Urrila, A. S., Leppänen, C., \& Polo-Kantola, P. (2015). Cardiac autonomic changes after 40 hours of total sleep deprivation in women. Sleep Medicine, 16(2), 250-257. doi:10.1016/j.sleep.2014.10.012

[50] Nozawa, A., \& Karita, K. (2010). Emotion spectrum analysis for a daily repetitive mental workload. Artificial Life and Robotics, 15(3), 316-319. doi:10.1007/s10015-010-0815-6

[51] Friedrichs, F., \& Yang, B. (2010). Camera-based drowsiness reference for driver state classification under real driving conditions. 2010 IEEE Intelligent Vehicles Symposium. doi:10.1109/ivs.2010.5548039

[52] Ronen, A., Oron-Gilad, T., \& Gershon, P. (2014). The combination of short rest and energy drink consumption as fatigue countermeasures during a prolonged drive of professional truck drivers. Journal of Safety Research, 49. doi:10.1016/j.jsr.2014.02.006

[53] Pushpalatha, N. (2014). Monitoring Behaviour Of Driver Through Sensors And Location Of Accident Through Wireless Technology. I-managers Journal on Embedded Systems, 3(1), 12-18. doi:10.26634/jes.3.1.2949

[54] Albalawi, H., Li, Y., \& Li, X. (2017). Training Fixed-Point Classifiers for On-Chip LowPower Implementation. ACM Transactions on Design Automation of Electronic Systems, 22(4), 1-18. doi:10.1145/3057275 
[55] Heart Rate Variability. (1994). European Heart Journal, 15(Suppl 2), 1-28. doi:10.1093/eurheartj/15.suppl_2.82

[56] Yung, C. (2011). Heart rate variability and underlying cardiovascular performance during spontaneous and controlled breathing (Unpublished master's thesis).

[57] How your heart works. (2018, April 30). Retrieved February 9, 2019, from https://www.nhsinform.scot/illnesses-and-conditions/heart-and-blood-vessels/about-theheart/understanding-how-your-heart-functions

[58] Molina, G. E., Fontana, K. E., Porto, L. G., \& Junqueira, L. F. (2016). Post-exercise heartrate recovery correlates to resting heart-rate variability in healthy men. Clinical Autonomic Research,26(6), 415-421. doi:10.1007/s10286-016-0378-2

[59] Abdi, R. M., Alhitary, A. E., Hay, E. W., \& Al-Bashir, A. K. (2018). Objective detection of chronic stress using physiological parameters. Medical \& Biological Engineering \& Computing, 56(12), 2273-2286. doi:10.1007/s11517-018-1854-8

[60] Schmidt, P., Reiss, A., Duerichen, R., Marberger, C., \& Laerhoven, K. V. (2018). Introducing WESAD, a Multimodal Dataset for Wearable Stress and Affect Detection. Proceedings of the 2018 on International Conference on Multimodal Interaction - ICMI 18. doi:10.1145/3242969.3242985

[61] Cho, D., Ham, J., Oh, J., Park, J., Kim, S., Lee, N., \& Lee, B. (2017). Detection of Stress Levels from Biosignals Measured in Virtual Reality Environments Using a Kernel-Based Extreme Learning Machine. Sensors, 17(10), 2435. doi:10.3390/s17102435

[62] Castaldo, R., Montesinos, L., Melillo, P., James, C., \& Pecchia, L. (2019). Ultra-short term HRV features as surrogates of short term HRV: A case study on mental stress detection in real life. BMC Medical Informatics and Decision Making, 19(1). doi:10.1186/s12911-019-0742-y

[63] Muaremi, A., Arnrich, B., \& Tröster, G. (2013). Towards Measuring Stress with Smartphones and Wearable Devices During Workday and Sleep. BioNanoScience, 3(2), 172183. doi:10.1007/s12668-013-0089-2

[64] Kanjo, E., Al-Husain, L., \& Chamberlain, A. (2015). Emotions in context: Examining pervasive affective sensing systems, applications, and analyses. Personal and Ubiquitous Computing, 19(7), 1197-1212. doi:10.1007/s00779-015-0842-3

[65] Venkatesan, C., Karthigaikumar, P., Paul, A., Satheeskumaran, S., \& Kumar, R. (2018). ECG Signal Preprocessing and SVM Classifier-Based Abnormality Detection in Remote Healthcare Applications. IEEE Access, 6, 9767-9773. doi:10.1109/access.2018.2794346

[66] Rukavina, S., Gruss, S., Hoffmann, H., Tan, J., Walter, S., \& Traue, H. C. (2016). Affective Computing and the Impact of Gender and Age. Plos One, 11(3).

doi:10.1371/journal.pone.0150584 
[67] Posada-Quintero, H. F., \& Bolkhovsky, J. B. (2019). Machine Learning models for the Identification of Cognitive Tasks using Autonomic Reactions from Heart Rate Variability and Electrodermal Activity. Behavioral Sciences, 9(4), 45. doi:10.3390/bs9040045

[68] Physiological Computing Gaming - Use of Electrocardiogram as an Input for Video Gaming. (2015). Proceedings of the 2nd International Conference on Physiological Computing Systems. doi:10.5220/0005244401570163

[69] Lima, R., Osório, D., \& Gamboa, H. (2019). Heart Rate Variability and Electrodermal Activity in Mental Stress Aloud: Predicting the Outcome. Proceedings of the 12th International Joint Conference on Biomedical Engineering Systems and Technologies. doi:10.5220/0007355200420051

[70] He, J., Li, K., Liao, X., Zhang, P., \& Jiang, N. (2019). Real-Time Detection of Acute Cognitive Stress Using a Convolutional Neural Network From Electrocardiographic Signal. IEEE Access, 7, 42710-42717. doi:10.1109/access.2019.2907076

[71] https://psychologycompass.com/blog/improve-focus/

[72] AutoSense: A Wireless Sensor System to Quantify Personal Exposures to Psychosocial Stress and Addictive Substances in Natural Environments. (n.d.). Retrieved from https://sites.google.com/site/autosenseproject/

[73] Heri. (2016, December 2). Managing Your Physical Condition with Heart Rate Variability. Retrieved from https://dailyhealthpoints.com/2016/12/01/managing-your-physical-conditionwith-heart-rate-variability/

[74] Pirbhulal, S., Zhang, H., Wu, W., Xu, L., \& Zhang, Y. T. (2017). Analysis of Efficient Biometric Index using Heart Rate Variability for Remote Monitoring of Obstructive Sleep Apnea. Neuropsychiatry, 07(06). doi: 10.4172/neuropsychiatry.1000280

[75] (2019, February 4). Retrieved from https://ylmsportscience.com/category/taper/page/2/

[76] (n.d.). HRV Expert System. Retrieved from http://thoughttechnology.com/index.php/hrvexpert-system.html

[77] (n.d.). HB100 Microwave Motion Sensor. Retrieved from https://pixelelectric.com/hb100$\underline{\text { microwave-motion-sensor/ }}$

[78] Spyder Wireless ECG - WEB Biotech Singapore. (2014, August 20). Spyder Wireless ECG: Wearable, Continuous ECG to Cloud utilising Smartphone Data Connectivity. Retrieved from https://www.youtube.com/watch? $\mathrm{v}=\mathrm{qXJ}$ f8bnsNd4 
[79] (n.d.). Industrial IoT Wireless Analog to Digital Converter ( 3.3V ADC). Retrieved from ht $T P_{\mathrm{s}}$ ://store.ncd.io/product/iot-long-range-wireless-analog-digital-converter/

[80] Kim, H.-G., Cheon, E.-J., Bai, D.-S., Lee, Y. H., \& Koo, B.-H. (2018). Stress and Heart Rate Variability: A Meta-Analysis and Review of the Literature. Psychiatry Investigation, 15(3), 235-245. doi: 10.30773/pi.2017.08.17

[81] Shaffer, F., \& Ginsberg, J. P. (2017). An Overview of Heart Rate Variability Metrics and Norms. Frontiers in Public Health, 5. doi:10.3389/fpubh.2017.00258

[82] McLaren, E. (2018, July 8). Is Stress Good for You? Retrieved from https://psychcentral.com/blog/is-stress-good-for-you/.

[83] Vanitha, L., Suresh, D.G., Chandrasekar, D.M., \& Punita, D.P. (2016). MENTAL STRESS LEVEL MEASUREMENT USING HRV ANALYSIS AND KNN CLASSIFIER WITH EFFICIENT VOTING SCHEME.

[84] Pattichis, S. Constantinos , and G. E. Andreas. "Autoregressive and Cepstral Analysis of motor unit action potential", Journal of Medical Engineering and Physics 21, pp. 405419, 1999.

[85] Burr, R. L., \& Cowan, M. J. (1992). Autoregressive spectral models of heart rate variability. Journal of Electrocardiology, 25, 224-233. doi:10.1016/0022-0736(92)90108-c

[86] Elsevier. (2011, September 22). Signals and Systems for Bioengineers. Retrieved from https://www.elsevier.com/books/signals-and-systems-for-bioengineers/semmlow/978-0-12384982-3.

[87] Delane, A., Bohorquez, J., Gupta, S., \& Schiavenato, M. (2016). Lomb algorithm versus fast fourier transform in heart rate variability analyses of pain in premature infants. 201638 th Annual International Conference of the IEEE Engineering in Medicine and Biology Society (EMBC). doi:10.1109/embc.2016.7590857

[88] Interpolation. (n.d.). Retrieved from https://dspguru.com/dsp/faqs/multirate/interpolation/.

[89] A. B Morgan, S.O Lilienfeld A meta-analytic review of the relation between antisocial behavior and neuropsychological measures of executive function Clinical Psychology Review, 20 (2000), pp. 113-136 (color stroop reference)

[90] Li, X., Yu, S., Chen, H., Lu, C., Zhang, K., \& Li, F. (2014). Cardiovascular autonomic function analysis using approximate entropy from $24-\mathrm{h}$ heart rate variability and its frequency components in patients with type 2 diabetes. Journal of Diabetes Investigation, 6(2), 227-235. doi:10.1111/jdi.12270 (poincare plot) 
[91] Du, J., Huang, J., An, Y., \& Xu, W. (2018). The Relationship between stress and negative emotion: The Mediating role of rumination. Clinical Research and Trials, 4(1).

doi:10.15761/crt.1000208

[92] Jang, E., Park, B., Park, M., Kim, S., \& Sohn, J. (2015). Analysis of physiological signals for recognition of boredom, pain, and surprise emotions. Journal of Physiological

Anthropology,34(1). doi:10.1186/s40101-015-0063-5

[93] Faraz, S., Ali, S. S., \& Adil, S. H. (2018). Machine Learning and Stress Assessment: A Review. 2018 3rd International Conference on Emerging Trends in Engineering, Sciences and Technology (ICEEST). doi:10.1109/iceest.2018.8643313

[94] Singh, A., Ganapathysubramanian, B., Singh, A.K., \& Sarkar, S. (2016). Machine Learning for High-Throughput Stress Phenotyping in Plants. Trends in plant science, 21 $2,110-124$.

[95] ML: Linear Regression. (2018, September 13). Retrieved from https:/www.geeksforgeeks.org/ml-linear-regression/

[96] Gandhi, R. (2018, May 17). Naive Bayes Classifier. Retrieved from https://towardsdatascience.com/naive-bayes-classifier-81d512f50a7c

[97] Murphy, K. P. (2012). Machine learning: a probabilistic perspective. Cambridge, MA: MIT Press.

[98] Aliyari, H., Sahraei, H., Daliri, M. R., Minaei-Bidgoli, B., Kazemi, M., Agaei, H., ... \& Dehghanimohammadabadi, Z. (2018). The beneficial or harmful effects of computer game stress on cognitive functions of players. Basic and clinical neuroscience, 9(3), 177.

[99] Roy, A., \& Ferguson, C. J. (2016). Competitively versus cooperatively? An analysis of the effect of game play on levels of stress. Computers in Human Behavior, 56, 14-20. doi:

10.1016/j.chb.2015.11.020

[100] Reinecke, L. (2009). Games and recovery: The use of video and computer games to recuperate from stress and strain. Journal of Media Psychology, 21(3), 126-142.

[101] Hsu, C.-H., Tsai, M.-Y., Huang, G.-S., Lin, T.-C., Chen, K.-P., Ho, S.-T., ... Li, C.-Y. (2012). Poincaré plot indexes of heart rate variability detect dynamic autonomic modulation during general anesthesia induction. Acta Anaesthesiologica Taiwanica, 50(1), 12-18. doi: 10.1016/j.aat.2012.03.002

[102] J. F. Schmidt, D. Romero and R. López-Valcarce, "Active Interference Cancellation for OFDM Spectrum Sculpting: Linear Processing Is Optimal," in IEEE Communications Letters, vol. 18, no. 9, pp. 1543-1546, Sept. 2014 
